SURFACE FLASHOVER UNDER RF AND UNIPOLAR

EXCITATION AT ATMOSPHERIC CONDITIONS

by

JOHN T. KRILE, B.S.E.E., M.S.E.E.

A DISSERTATION

IN

\title{
ELECTRICAL ENGINEERING
}

Submitted to the Graduate Faculty

of Texas Tech University in

Partial Fulfillment of

the Requirements for

the Degree of

DOCTOR OF PHILOSOPHY

Approved

Dr. Andreas Neuber

Chairperson of the Committee

Dr. Hermann Krompholz

Dr. Magne Kristiansen

Dr. Thomas Gibson

Accepted

John Borrelli

Dean of the Graduate School

May, 2006 
(C) 2006

JOHN KRILE

All Rights Reserved 


\section{ACKNOWLEDGEMENTS}

I would like to thank my committee for their support and guidance during this project. I would especially like to thank Dr. Neuber who served as chairman of my committee. He has always believed in me and kept me on track. Dr. Krompholz has provided input and guidance without which the project would not have been a success. I would like to give special recognition to Dr. Gibson for allowing me to use the Gamera cluster, and for his "wild ideas" that proved to be incredibly helpful. And finally I would like to thank Dr. Kristiansen, without whom there would not be a Pulsed Power lab where I have learned so much.

Special thanks are also owed to the staff of The Center for Pulsed Power and Power Electronics at Texas Tech. Specifically, I would like to thank Dino Castro, Shannon Gray, Danny Garcia, Marie Byrd, and all of the undergraduates that assisted me on this project. I could not have done it without these individuals. I would also like to thank my colleagues including Greg Edmiston, Shad Holt, Mike Butcher, and John Walter, who were always ready to lend a helping hand. I cannot begin to describe how much I value your assistance and your friendship.

Finally, I wish to express supreme gratitude to my wife, Melissa, for her encouragement, support, companionship, and unconditional love. 


\section{TABLE OF CONTENTS}

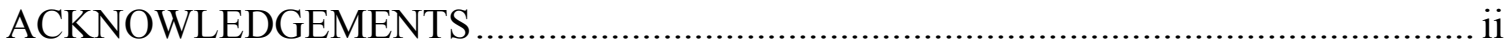

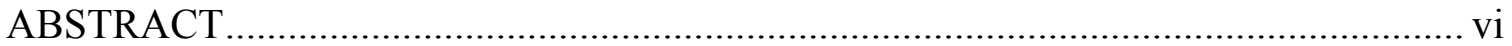

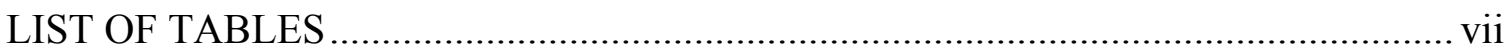

LIST OF FIGURES ……………………………….......................................... vii

CHAPTERS

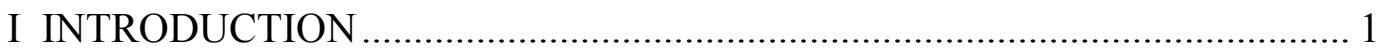

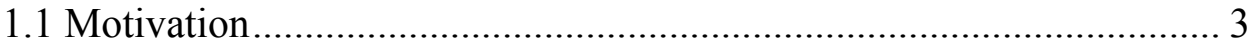

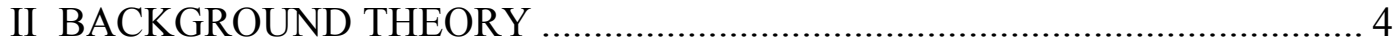

2.1 Electron Emission Processes.................................................................. 4

2.1.1 Field Electron Emission.......................................................... 4

2.1.2 Photoelectric Emission............................................................ 6

2.1.3 Secondary Electron Emission .................................................. 7

2.2 Electron Amplification Processes ........................................................ 8

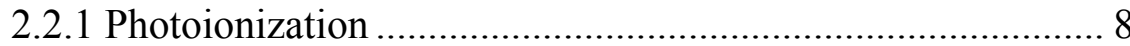

2.2.2 Collision Processes ............................................................... 9

2.3 Electron Loss Processes ................................................................... 10

2.3.1 Electron Attachment ............................................................ 11

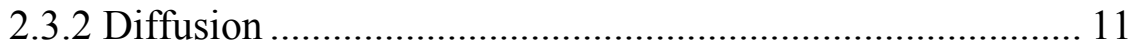

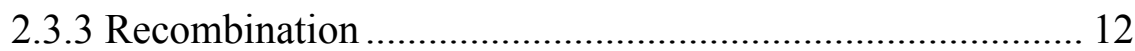

2.3.4 Collision Losses................................................................ 13

2.3.5 Excitation Losses .............................................................. 13

2.4 Summary of Collision Cross Sections …………………………....... 17

2.5 Volume Breakdown Mechanisms ..................................................... 18

2.5.1 Townsend Mechanism ........................................................ 19

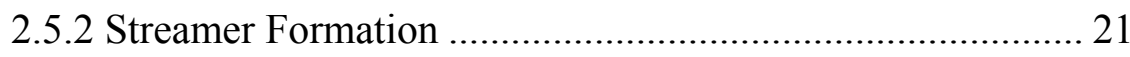

2.5.3 Secondary Electron Emission Avalanche ............................. 24

2.6 Previous Research of Unipolar Flashover.......................................... 25 
2.7 Previous Research of HPM Breakdown ............................................ 27

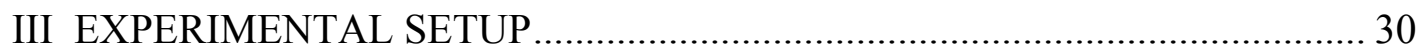

3.1 Unipolar Testing Apparatus .............................................................. 30

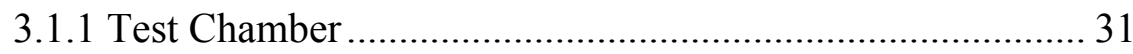

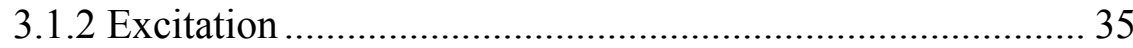

3.1.3 Environmental Control....................................................... 38

3.1.4 Electrode Design................................................................ 39

3.1.4.1 Hemispherical Design ............................................ 39

3.1.4.2 Angled Design ....................................................... 41

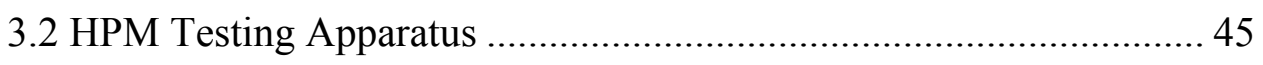

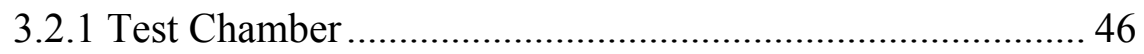

3.2.2 Flange/Window Design .................................................... 47

3.2.3 Environmental Control........................................................ 53

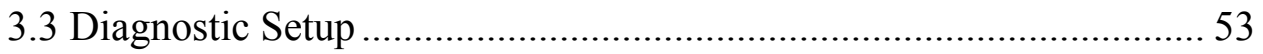

3.3.1 Current Sensors .............................................................. 54

3.3.2 RF Power Measurements .................................................... 56

3.3.3 Optical Diagnostics ........................................................... 57

3.3.3.1 Collection Apparatus …………………………..... 57

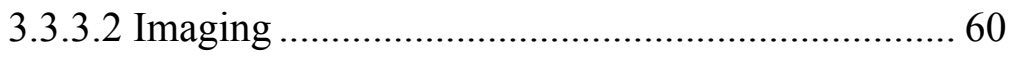

3.3.3.3 Photo Multiplier Tubes ........................................... 60

3.3.3.4 Spectrograph .......................................................... 61

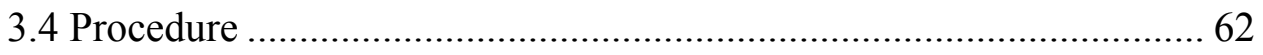

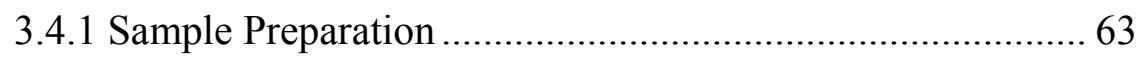

3.4.2 Electrode / Flange Preparation................................................ 63

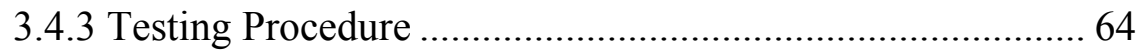

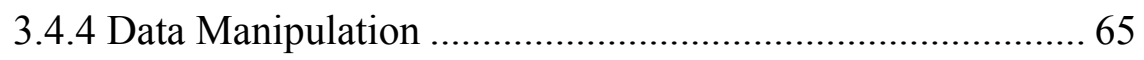

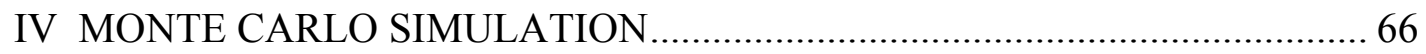

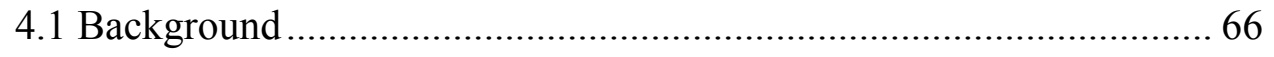

4.2 Program Operation........................................................................ 73 
4.2.1 Program Control..................................................................... 73

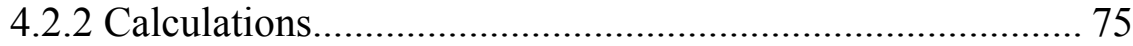

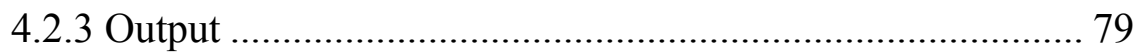

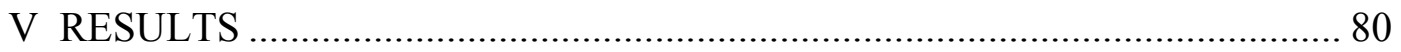

5.1 Arc Channel Formation.................................................................... 82

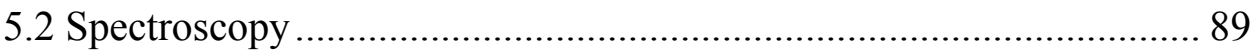

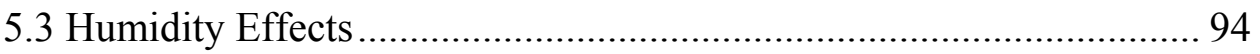

5.4 Monte Carlo Simulation.................................................................... 96

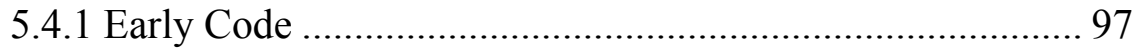

5.4.2 Sensitivity Testing .......................................................... 100

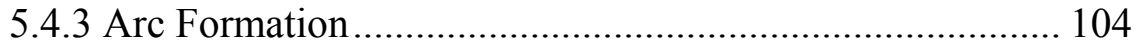

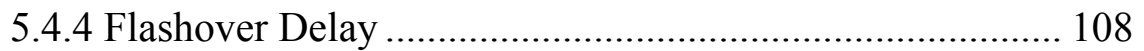

5.4.5 Electron Energy Distribution ............................................. 115

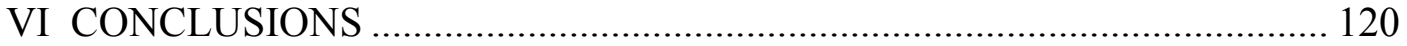

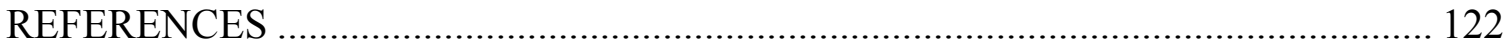




\begin{abstract}
In vacuum environments surface flashover events driven by $\mathrm{RF}$ (f $<10 \mathrm{GHz}$ ) and unipolar excitation have been shown to have virtually identical dominant mechanisms. Similarities between RF (representing high-power microwave, HPM, window breakdown on the high pressure side) and unipolar surface flashover are expected in an atmospheric environment as well. Extensive testing has already been completed for both DC and pulsed unipolar flashover with several dominant processes identified. Further testing with a setup capable of HPM driven surface flashover are conducted to identify similar dominant processes.
\end{abstract}

The two separate experimental setups, utilized to investigate both unipolar flashover and RF window flashover under atmospheric conditions, enable controlling excitation, temperature, pressure, humidity, and type of gas present, all under similar electric fieldsurface geometry. In order to ensure the conditions are as matched as possible, the local electric field at the flashover initiating points has been numerically calculated in detail for all test geometries. In addition a Monte Carlo type electron motion simulation program was created to further isolate the individual processes leading to flashover.

For both RF and unipolar pulsed excitation, the flashover dynamics are changed by the application of UV light to the dielectric surface. A UV pre-pulse has a distinct impact on the arc's path and a tendency to decrease the hold-off electric field. The effect of humidity on the hold-off electric field for both pulsed unipolar and RF excitations, along with temporally resolved emission spectroscopy of the flashover event, are discussed. 


\section{LIST OF TABLES}

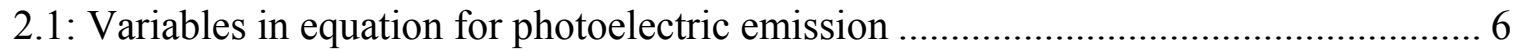

2.2: Variables in the Gerald Dionne model ….......................................................... 7 


\section{LIST OF FIGURES}

2.1: Diagram of energy required to free one electron from a metal surface $[16]$............. 5

2.2: Graph of total SEE coefficient versus the energy of incident electron...................... 8

2.3: Ionization collision cross sections for nitrogen (black) and oxygen (red) from [25] 10

2.4: Attachment collision cross section for oxygen [26] ........................................... 11

2.5: Elastic collision cross sections for nitrogen (black) and oxygen (red) from [27] and

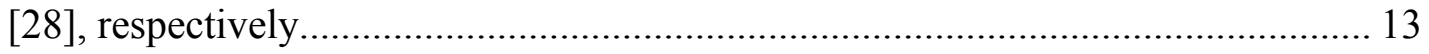

2.6: Vibrational collision cross sections for nitrogen. The excited vibrational state for the electronic ground state along with the energy lost by the impacting electron is

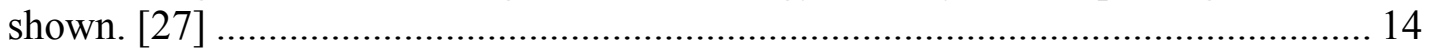

2.7: Vibrational collision cross sections for oxygen. The excited vibrational state along with the energy lost by the impacting electron is shown. [28] ................................ 15

2.8: Electronic collision cross sections for nitrogen. The electronic transition as well as the energy lost by the impacting electron is shown. [29] .................................... 15

2.9: Electronic collision cross sections for oxygen. The energy lost by the impacting

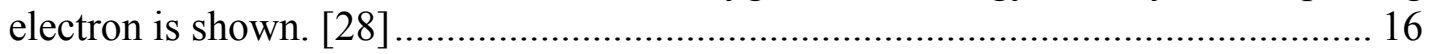

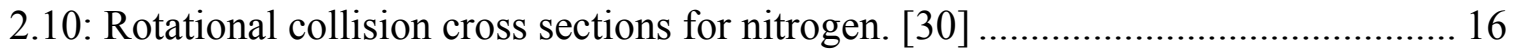

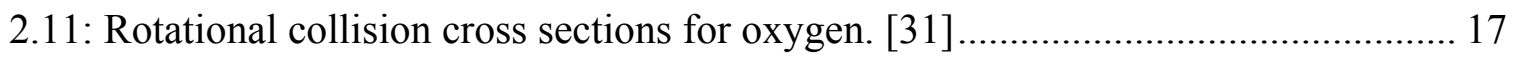

2.12: Townsend V-I curve for gap current vs. potential [36] ...................................... 19

2.13: Diagram of electrons and ions in an electron avalanche, showing ion $(+)$ and

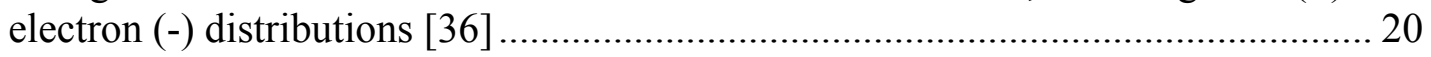

2.14: Diagram of early stages in the streamer development, showing primary and secondary electron avalanches, space charges caused by ions and electrons, as well

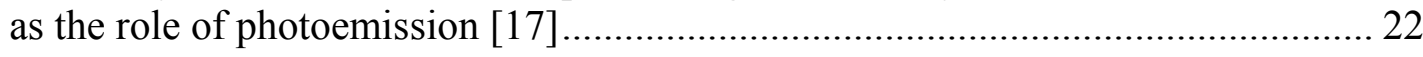

2.15: Diagram of late stages in the streamer development, including the development of the ionization channel through continued electron avalanche additions and the final completed channel 
2.16: Diagram of secondary electron emission avalanche [2]

3.1: Overview of entire unipolar setup. Shown for pulsed excitation, for DC excitation the lines are charged to equal and opposite voltages. Environmental controls are omitted 31

3.2: AutoCad drawing of testing chamber with feedthroughs and hemispherical electrodes, removable mesh outer conductor not shown [47]................................. 32

3.3: Picture of testing chamber with the feedthroughs, current sensors, and camera lens shown 33

3.4: Cross-section of transition, also called the feedthrough, from charging to test chamber with key component identification [47] 34

3.5: Comparison of electric field strengths at electrode tips and at dielectric feedthrough, with each electrode at $\pm 20 \mathrm{kV}$, respectively 35

3.6: Schematic of HV pulser. The center triggering electrode of the spark gap is floated to half the gap potential via the R_Divide resistor 36

3.7: Picture of the high voltage pulser setup. 37

3.8: Schematic diagram of humidification system. Purge valves are used to force out any humid gas from the system after use.

3.9: Drawing of hemispherical electrodes and attached polycarbonate surface

3.10: Magnitude plot of electric field near the hemispherical electrode tips, gap distance of $6.5 \mathrm{~mm}$, electrodes charged to $\pm 20 \mathrm{kV}$ 41

3.11: Vector plot of electric field near the hemispherical electrode tips, gap distance of $6.5 \mathrm{~mm}$, electrodes charged to $\pm 20 \mathrm{kV}$

3.12: Picture of angled electrodes installed in the test chamber with a flat polycarbonate surface.

3.13: Vector plot of the electric field from a side view of the angled electrodes, gap distance of $8.6 \mathrm{~mm}$, electrodes charged to $\pm 20 \mathrm{kV}$

3.14: Magnitude plot of the electric field from a side view of the angled electrodes, gap distance of $8.6 \mathrm{~mm}$, electrodes charged to $\pm 20 \mathrm{kV}$. 43

3.15: Image of angled electrodes inserted into grooved surface. 44 
3.16: Magnitude plot of the electric field from a side view of the angled electrodes with a grooved surface, gap distance of $8.4 \mathrm{~mm}$, electrodes charged to $\pm 20 \mathrm{kV}$ 44

3.17: Vector plot of the electric field from a side view of the angled electrodes with a grooved surface, gap distance of $8.4 \mathrm{~mm}$, electrodes charged to $\pm 20 \mathrm{kV}$ 45

3.18: Overall system diagram for HPM surface flashover testing. Power measurement diagnostics via a pair of directional couplers in front of the load and after the HPM

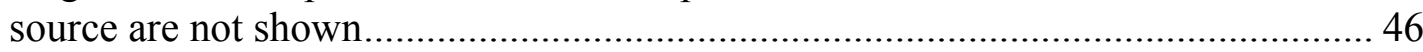

3.19: Close up view of the exit flange geometry utilized to eliminate metallic conductors from the flashover location [15] 48

3.20: Cross section of intermediary gasket design A. The gasket brass gasket is shown between the exit flange and the polycarbonate surface 49

3.21: Cross section of intermediary gasket design B. The gasket brass gasket is shown between the exit flange and the polycarbonate surface

3.22: Field magnitude profile of intermediary gasket (A) of Figure 3.20 from Ansoft HFSS simulations 50

3.23: Field vector plot of intermediary gasket (A) of Figure 3.20 from Ansoft HFSS simulations 50

3.24: Field magnitude profile of intermediary gasket (B) along the A-A cut line of Figure 3.21 from Ansoft HFSS simulations. 51

3.25: Field vector plot of intermediary gasket (B) along the A-A cut line of Figure 3.21 from Ansoft HFSS simulations 51

3.26: E-field components along the polycarbonate window surface as a function of vertical location along the A-A cut line of intermediary gasket B (c.f. Figure 3.21) from Ansoft HFSS simulations, 3.25MW incident power [15] ................................ 52

3.27: Diagram of the traveling wave current sensors [48] ............................................ 55

3.28: Diagram of capacitive voltage divider implemented on a coaxial geometry .......... 56

3.29: Reference image of front of sample with pseudo-color image of a flashover overlaid, spectra is integrated from 3 regions along the arc path as denoted by the

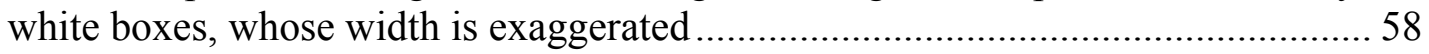

3.30: Fiber optics setup for spatially resolving light emission in the flashover gap......... 59 
3.31: Demonstration of difference in imaging and non-imaging spectrographs, imaging spectrographs have specially designed mirrors to maintain vertical resolution 62

3.32: Schematic of multi-track spectrograph operation, For the HPM setup direct imaging with a lens is used in place of the fiber optic cables. 62

4.1: Description of force felt by and electron due to an external electric field. 67

4.2 The cumulative collision frequency is the addition the collision frequencies of all considered collisions as stated in equation (4.18). . 70

4.3: Cumulative collision frequency is the addition the collision frequencies of all considered collisions plus a null collision frequency yielding a constant cumulative collision frequency 71

4.4: Distribution of calculated collision times given a 2 collision/ps cumulative collision frequency and 100,000 iterations. 72

4.5: Illustration of the determination of collision type using a uniformly distributed random number and the collision frequencies at a give velocity 73

4.6: Parameter list for giving the simulation control variables. The variables changed most often are denoted by the blue arrows 74

4.7: Collision cross section list for identification of cross section to be used. The ratio of gases present in the volume is also controlled here 75

4.8: Program flowchart illustrating the steps involved in calculating each write time..... 76

4.9: Depiction of the definition of deflection, $\theta$, and roll, $\Phi$, with $\mathrm{v}$ being the original direction of the velocity vector and $\mathrm{v}^{\prime}$ the resultant vector 77

5.1: Current and voltage waveforms of a typical pulse with flashover. The top plot shows the camera trigger pulse. The middle plot shows the current rise at flashover. The bottom plot depicts the incident pulse and the voltage drop at flashover. 81

5.2: Power and luminosity waveforms for a typical HPM surface flashover. The top graph shows the incident power (black), the transmitted power (blue) and the reflected power (red). The bottom graph shows the luminosity in green and the amplified luminosity in orange [15]. 82

5.3: Side-on pseudo color intensity plot of main breakdown in air with a polycarbonate surface and angled electrode, $8.1 \mathrm{~mm}$ gap. Pulse voltage, $\mathrm{V}_{\mathrm{p}}=25 \mathrm{kV}$. 83 
5.4: Side-on pseudo color intensity plot of main breakdown in nitrogen with a polycarbonate surface and angled electrode, $8.1 \mathrm{~mm}$ gap. Pulse voltage, $\mathrm{V}_{\mathrm{p}}=25 \mathrm{kV}$

5.5: Side-on pseudo color intensity plot of main breakdown in air with a polycarbonate surface and angled electrode, $8.1 \mathrm{~mm}$ gap. Pulse voltage, $\mathrm{V}_{\mathrm{p}}=25 \mathrm{kV}$ 84

5.6: Side-on pseudo color intensity plot of main breakdown in nitrogen with a polycarbonate surface and angled electrode, $8.1 \mathrm{~mm}$ gap. Pulse voltage, $\mathrm{V}_{\mathrm{p}}=25 \mathrm{kV}$

5.7: Side-on pseudo color intensity plot of main breakdown in air with a polycarbonate surface under HPM excitation 85

5.8: Side-on pseudo color intensity plot of main breakdown in nitrogen with a polycarbonate surface under HPM excitation. 85

5.9: Series of pseudo-color images showing the development of a flashover with time referenced to the rise in the luminosity signal, all images taken in air at 155 torr ... 86

5.10: Side-on pseudo color intensity plot of main breakdown in nitrogen under HPM excitation. Time indicates delay from UV illumination of the surface to application of HPM excitation. 88

5.11: Optical emission spectra collected in air at 680 torr with unipolar excitation (top, bottom: close to electrodes, center: in between). Note the $\mathrm{H}_{\alpha}$ line at $656 \mathrm{~nm}$ 89

5.12: Optical emission spectra collected in nitrogen at 680 torr with unipolar excitation (top, bottom: close to electrodes, center: in between)

5.14: Optical emission spectra collected in air at 155 torr with HPM excitation 91

5.15: Optical emission spectra collected in nitrogen at 155 torr with HPM excitation .... 92

5.16: Comparison Optical emission spectra collected at 155 torr with HPM excitation, $40 \mathrm{~ns}$ after arc initiation (top, red), with $\mathrm{N}_{2}$ calculated spectra shown (bottom, grey) at a vibrational temperature of $4000 \mathrm{~K} \pm 500 \mathrm{~K}$, rotational temperature is set to room temperature, the vibrational transitions responsible for the various band heads are labeled [15] 92 
5.17: Optical emission spectra collected at 155 torr with HPM excitation, $\sim 40$ ns after arc initiation. Scale is adjusted relative to the dominant peak at $400 \mathrm{~nm}$ for highlighting the increased UV content in air environment. 93

5.18: Breakdown voltage vs. relative humidity in air (red circles), and nitrogen (black triangles) for unipolar excitation [47] 95

5.19: Pressure vs. delay time in air at low humidity $(<15 \% \mathrm{RH})$, triangles, and high humidity $(>85 \% \mathrm{RH})$, circles, for HPM excitation of $3 \mathrm{MW}(\sim 13.5 \mathrm{kV} / \mathrm{cm}) \ldots \ldots . . .96$

5.20: Drawing of WR284 exit flange (brown), polycarbonate sample (blue), and the simulation area (green box). The coordinate system is defined as shown 97

5.21: Electron energy distribution generated by the 1D simulation in pure nitrogen, 1000 initial electrons, $5.75 \mathrm{kV} / \mathrm{cm}$ electric field with a frequency of $2.85 \mathrm{GHz}$ 99

5.22: Electron energy distribution generated by the EEDF calculator in pure nitrogen, $5.75 \mathrm{kV} / \mathrm{cm}$ electric field with a frequency of $2.85 \mathrm{GHz}$. 99

5.23: Total electron count verses simulation time in a nitrogen environment with the real elastic cross section (blue), half the elastic cross section (red) and double the elastic cross section (green) 100

5.24: Total electron count verses simulation time in a nitrogen environment with the 16 seed electrons (green), 1600 seed electrons (blue) and 16000 seed electrons (red) 102

5.25: Demonstration of sensitivity of the simulation to the initial placement of electrons within the volume, each curve represents the seed value of the random number generator varied by the given factor. 103

5.26: Flashover delay times in both air and nitrogen environment for varying frequencies at 100 torr under $15 \mathrm{kV} / \mathrm{cm}$ excitation 104

5.27: Electron cloud formation in a nitrogen environment with 16 seed electrons 106

5.28: Comparison of the electric field scaling function (top, red) and the actual ramp up (bottom, black) associated with the HPM source 106

5.29: Electron cloud formation in an air environment with 1600 seed electrons 107

5.30: Demonstration of method used to extrapolate flashover delay times, solid portion of the curves are calculated at various pressures and fields, dashed portion of the lines are curve fits 109 
5.31: Calculated Flashover delay time versus electric field in air and nitrogen at 200 torr excited with an ideal square pulse

5.32: Normalized electric field verses delay time multiplied by pressure in air. Curve for volume breakdown (blue) from Gould and Roberts compared to data collect on current test setup (black circles 3MW or $18 \mathrm{kV} / \mathrm{cm}$ pulse and black triangles for $4.5 \mathrm{MW}$ or $25 \mathrm{kV} / \mathrm{cm}$ pulse). The red line represents a curve fit of the current data. [15] 111

5.33: Normalized electric field verses delay time multiplied by pressure in air. The graph shows volume breakdown curve from Gould and Roberts (blue line), data collect on the current test setup (green circles), simulated data (orange diamonds), and a curve fit of the all experimental data in air (red line)..... 113

5.34: Normalized electric field verses delay time multiplied by pressure in nitrogen. The graph shows volume breakdown curve from Gould and Roberts (blue line), data collect on current test setup (green circles), simulated data with 1600 initial electrons (orange triangles), simulated data with 16 initial electrons (orange squares), and a curve fit of the all experimental data in air (red line) 114

5.35: Normalized electric field verses delay time multiplied by pressure in both an air (green squares) and nitrogen (blue squares) environment. The graph also shows volume breakdown curve from Gould and Roberts (dark blue line), and a curve fit of the all experimental data in air (red line) 115

5.36: Snap shot of the electron energy distribution function at several time steps in an air environment at 125 torr, with a microwave period of $350 \mathrm{~ns}$. 116

5.37: Comparison of EEDF's for an air environment generated by the simulation and by an alternate EEDF program, the simulation EEDF is averaged over 2 periods with zero net electron gain 118

5.38: Comparison of EEDF's for an nitrogen environment generated by the simulation and by an alternate EEDF program, the simulation EEDF is averaged over 2 periods with zero net electron gain 118

5.39: Comparison of EEDF's for an air and nitrogen environment generated by the simulation, the EEDF's are averaged over 2 periods with zero net electron gain .. 119 


\section{CHAPTER I}

\section{INTRODUCTION}

There is a large body of empirical data on unipolar surface flashover in the atmosphere. This information is used to create design rules for the power distribution industry. The physics of the involved processes, however, is generally unknown [1 - 3]. Most studies have focused on varying the environmental conditions, such as moisture and dust settling on the insulator, with only the flashover voltage as the primary measured quantity. Very little effort has been put into temporally resolving the surface flashover

processes, for instance, by measuring the flashover time delay, or applying advanced diagnostics, such as imaging with nanosecond exposure time. Even less studied is the surface flashover caused by high-power microwaves (HPM's) on the air side of a window, which is needed for maintaining vacuum in the HPM source itself, while radiating the power into the outside environment.

Both the unipolar and RF surface flashover cases have been studied in detail under vacuum conditions and have been found to have the same dominant mechanisms $[4,5]$. It is postulated that similarities exist for unipolar and RF surface flashover at atmospheric conditions. As in the unipolar excitation, the only reasonable way to model HPM window breakdown or surface flashover is a direct simulation of the discharge, considering all the elementary processes contributing to the flashover event. In this fashion, some of the processes were identified for unipolar excitation [6]. By comparing these processes to those in HPM excitation, it should be possible to advance the understanding of the 
phenomena common to both cases. In addition, a Monte Carlo type simulation should yield further information about the dominant underlying processes. All experiments were performed at The Center for Pulsed Power and Power Electronic at Texas Tech University. The center has a great deal of experience with surface flashover and breakdown in general. Diagnostic systems for observing unipolar breakdowns were first developed at Texas Tech by Hegeler [7, 8]. Later, Neuber developed a system for testing surface flashover in vacuum at cryogenic temperatures [9]. The present unipolar experimental setup design is based on the cryogenic test apparatus with modifications made to accommodate atmospheric temperatures and pressures.

Relatively few attempts have been made to study the mechanisms of RF flashover of a dielectric surface in atmospheric pressures $[10,11]$. Diagnostic systems for observing HPM flashover were first developed at Texas Tech by Hatfield [12] and later expanded upon by Neuber and Hemmert $[4,6,13]$. The HPM source has been attached to a new test setup designed by Edmiston and Krile [14, 15] that allows for the control of environmental variables as in the unipolar setup. The setups are able to produce and closely monitor flashover across various gap distances and insulator geometries at atmospheric conditions with different humidities. This allows the conditions to be changed in order to isolate the roles of individual processes. In order to closely monitor the flashover event, current, voltage, power, luminosity, spatially resolved light emission, and optical emission spectra are all measured with nanosecond to sub-nanosecond resolution. 


\subsection{Motivation}

The limits of the applicability of dc, ac, or pulsed high voltage are determined by breakdown along insulators or insulating support structures. This includes unintentional high voltage situations, such as a lightning strike on a building or a surge caused by a surge in the power grid. In order to obtain safety guidelines for a wider range of insulator flashover events a more complete understanding of the underlying processes is necessary. This is especially important since it easily applies to the protection of sensitive materials, including explosives and detonators.

Microwave breakdown phenomena have been a topic of interest in many areas of applied physics for some time. Since the need to transmit high power microwave (HPM) radiation is paramount in communication systems as well as the development of technologies such as directed-energy systems and high power radar applications, the breakdown phenomena which limit the generation and transportation of this radiation deserve considerable attention. Surface flashover of windows in High Power Microwave (HPM) systems is also a major limiting factor for transmitting HPM into the atmosphere.

In both cases the advancement of the technology hinges on the understanding and mitigation of window flashover events. 


\section{CHAPTER 2}

\section{BACKGROUND THEORY}

A basic knowledge of the fundamental processes underlying the flashover event is necessary. While detailed discussions of all the processes are available in literature [16 18], they will be listed and briefly summarized here. These processes are grouped into three categories: electron emission, electron amplification, and electron loss processes. In addition to a brief description of the process, the relevant collision cross sections will also be discussed as they relate to the Monte Carlo simulation.

\subsection{Electron Emission Processes}

Electron emission can play an important role in breakdown, especially when electrodes or a dielectric surface are present such as in the unipolar case. There are several types of electron emission that have been well documented; however only a few play a role in the processes of surface flashover. These major contributors include electric field emission, photoemission, and secondary electron emission. All emissions involve overcoming of the work function, $\Phi_{\text {Surface, }}$ or the amount of energy required to free an electron from the surface of a material.

\subsubsection{Field Electron Emission}

The presence of an external electric field changes the amount of energy required to release an electron from the surface of an electrode $[19,20]$. As an electron leaves the 
surface, its electric field can be approximated as that between a point charge and an equipotential plane. By using Coulomb's law and integrating, the potential energy, $\mathrm{W}_{\mathrm{e}}$, of the electron can be determined at some distance, $\mathrm{x}$, from the surface. The accelerating electric field perpendicular to the surface with a magnitude of $\mathrm{E}$ also produces a potential energy, $\mathrm{W}_{\mathrm{f}}$. The individual energies and their combined effect, $\mathrm{W}_{\mathrm{t}}$, can be seen in Figure $2.1[16]$.

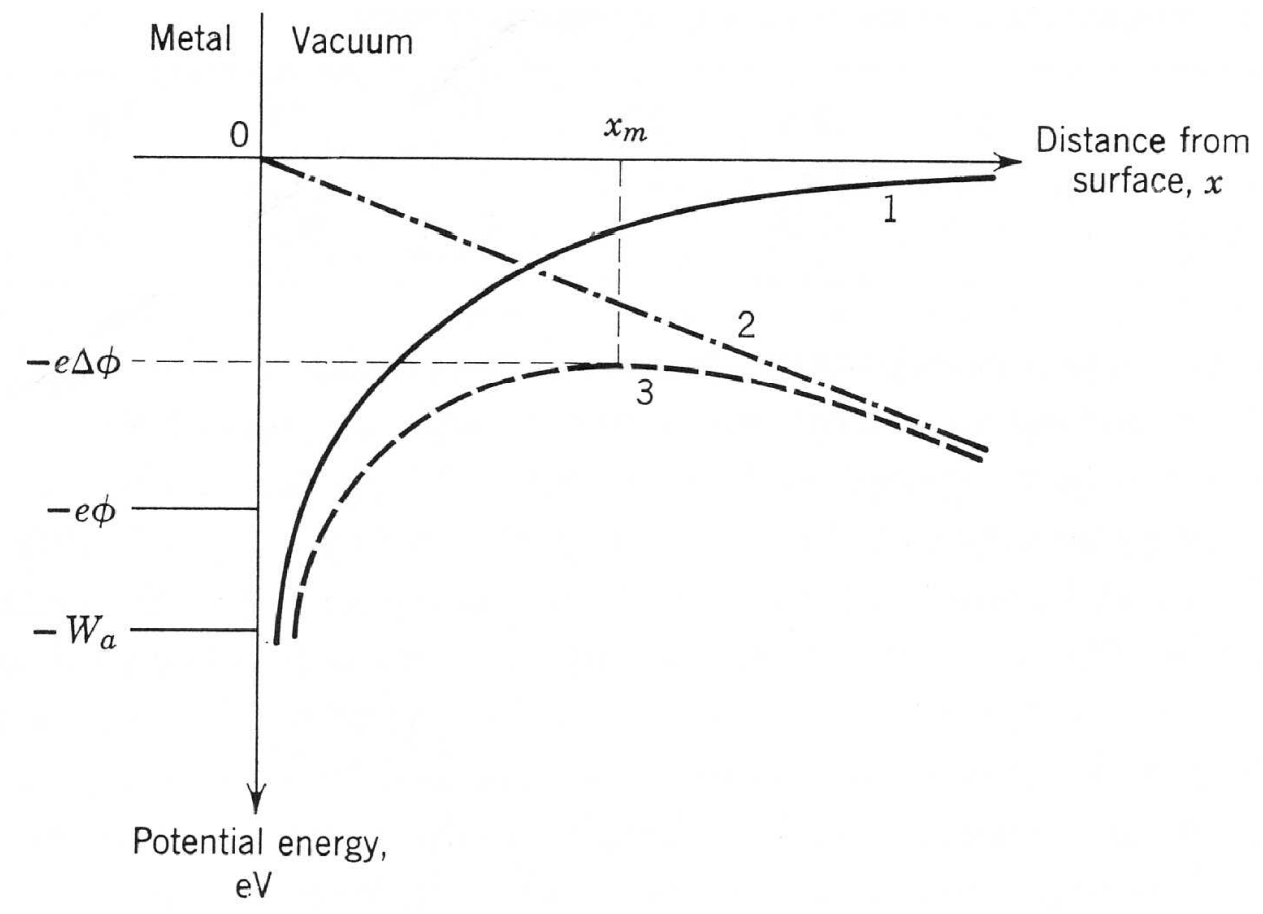

The lowering of the potential barrier by an external field: curve 1 -energy curve with no external field, $-W=e^{2} / 16 \pi E_{0} x$; curve 2energy due to applied field, $-W_{f}=e E x$; curve 3 -total energy curve, $W_{t}=W_{f}+W_{e}$.

Figure 2.1: Diagram of energy required to free one electron from a metal surface [16]

As the applied field is increased, the total energy curve is bent over further, and an effect called the tunneling effect begins to apply. The tunneling effect is the probability 
that an electron with energy less than the barrier can "tunnel" through to the other side of the barrier. Fowler and Nordheim, using quantum mechanics, were the first to describe this effect [20].

\subsubsection{Photoelectric Emission}

Photoelectric emission is the process of liberating electrons from a surface by irradiating it with photons. An electron will be emitted from the surface only if the incident photons have enough energy to overcome the work function of the surface. Any excess energy of the photons will be converted to kinetic energy of the emitted electron. The equation describing photoelectric emission shows that the energy of the photon is equal to the work required to free the electron and the kinetic energy given the electron:

$$
h v=\frac{1}{2} \cdot m \cdot v^{2}+e \Phi
$$

Table 2.1: Variables in equation for photoelectric emission

\begin{tabular}{|c|l|}
\hline$h\left(\mathrm{~J}^{*} \mathrm{~s}\right)$ & Planck's constant \\
\hline$v(1 / \mathrm{s})$ & Frequency of photon \\
\hline$m(\mathrm{~kg})$ & Mass of electron \\
\hline $\mathrm{v}(\mathrm{m} / \mathrm{s})$ & Velocity of electron \\
\hline$e(\mathrm{C})$ & Charge of an electron \\
\hline$\Phi(\mathrm{V})$ & Work function \\
\hline
\end{tabular}

The photoelectric effect is characterized by its quantum yield, which is the number of electrons freed per incident photon. The quantum yield for a given surface, $\gamma_{v}$, is generally $\sim 10^{-3}$, in the near-UV region and $\sim 10^{-2}-10^{-1}$ in the far-UV region $[21-23]$. 


\subsubsection{Secondary Electron Emission}

Secondary electron emission is the process of liberating a secondary electron from a solid surface by impacting it with another electron called the primary. For every primary electron incident on the solid's surface there is a certain probability that a secondary electron will be emitted from the surface. The yield, called $\delta$, is the ratio of secondary electrons emitted for every primary electron. An important factor in the release of secondary electrons is the energy of the incident primary electrons, as well as the penetration depth of the primary electron. Other factors, such as the material absorption coefficient and probability of escape, also play an important role in determining the yield. These factors are shown by the Gerald Dionne model [24]:

$$
\delta=\left(\frac{B}{\xi}\right) \cdot\left(\frac{A n}{\alpha}\right)^{1 / n} \cdot(\alpha d)^{1 /(n-1)} \cdot\left(1-e^{-\alpha d}\right)
$$

Table 2.2: Variables in the Gerald Dionne model

\begin{tabular}{|c|l|}
\hline$\delta$ & SEE Yield \\
\hline$B$ & Secondary Escape Probability \\
\hline$\xi$ & Secondary-Electron Excitation Energy \\
\hline$A$ & Primary Electron Absorption Constant \\
\hline$\alpha$ & Secondary-Electron Absorption Constant \\
\hline$n$ & Power-Law Exponent \\
\hline$d$ & Maximum Penetration Depth \\
\hline
\end{tabular}

The curve shown in Figure 2.2 shows the yield of secondary electrons vs. the energy of the primary electrons. The curve has two "cross-over" points where the yield is equal to 1 . The reason that the curve has a maximum at a certain level is that the electrons with 
too large an energy will penetrate deep into the surface and the secondary electrons will have a low probability of reaching and escaping the surface.

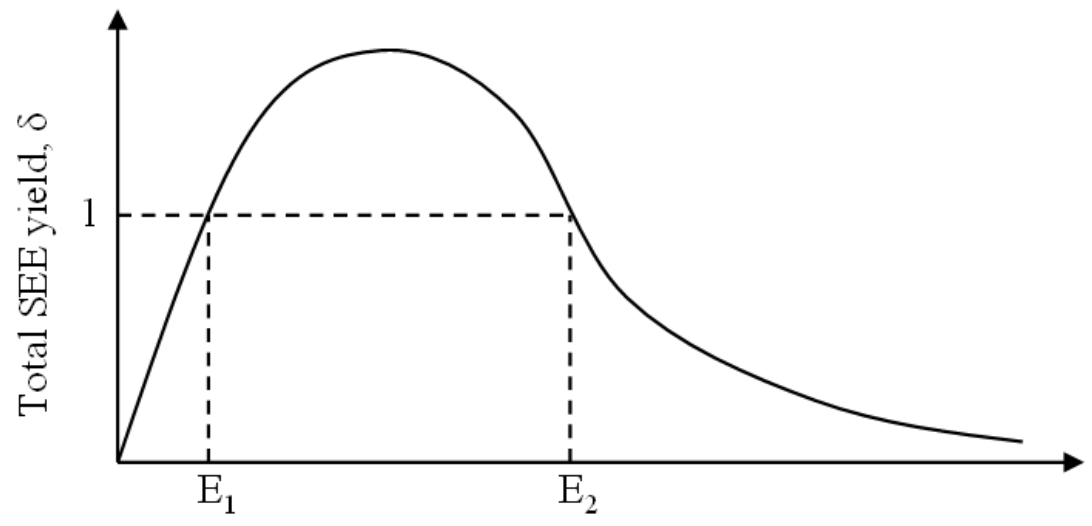

Energy of Incident Electron

Figure 2.2: Graph of total SEE coefficient versus the energy of incident electron

\subsection{Electron Amplification Processes}

A key factor in the formation of a discharge is electron multiplication, which can occur through a number of processes including electron-molecule collisions and photoionization. Electrons, accelerated by the external electric field, impact the molecules in the surrounding gas, occasionally resulting in ionization and the creation of a secondary electron. In the same way photons striking molecules can also cause ionizations. This section will present some of the most important amplification processes.

\subsubsection{Photoionization}

Another source of electrons is photoionization, in which a photon is absorbed by a molecule and an electron is freed. This is primarily true of UV radiation which has been experimentally shown to ionize almost any gas [16]. The energy of an impacting photon 
must be greater than the ionization energy, $W_{i}$. The conditional equation for ionization can be written in the form shown in equation (2.3), where $\mathrm{c}$ is the speed of light, $h$ is Planck's constant, and $\lambda$ is the wavelength of the incident photon.

$$
\lambda \leq \frac{c \cdot h}{W_{i}}
$$

\subsubsection{Collision Processes}

The presence of an electric field will produce a force, parallel to the field direction, on any charged particles such as ions and electrons. This force increases the velocity of these charged particles and therefore increases the likelihood of electron-molecule collisions up to a certain limit. The effective area for a collision is given by the collision cross sections as a function of particle energy or velocity. This is especially true of electrons which experience a greater acceleration due to their smaller mass.

Collisions which result in the creation of a new electron are considered ionizing collisions. In this case the incident electron must have sufficient energy to overcome the ionization energy of the molecule, $\sim 16.5 \mathrm{eV}$ for nitrogen and $\sim 12.5 \mathrm{eV}$ for oxygen [25]. The energy remaining after the ionization potential is taken from the incident electrons' initial energy is split between the deflected incident electron and the generated electron. The collision cross sections for oxygen and nitrogen are shown in Figure 2.3. 


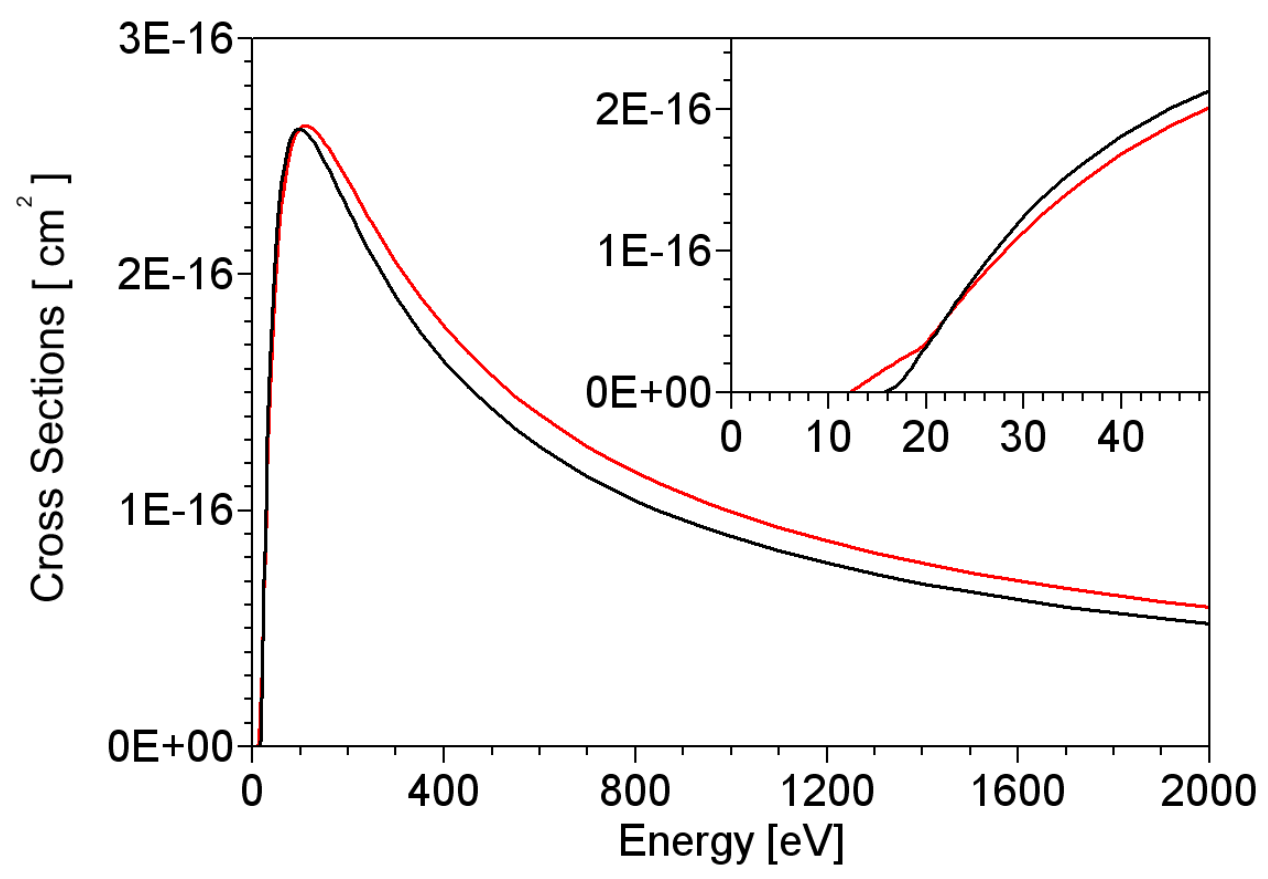

Figure 2.3: Ionization collision cross sections for nitrogen (black) and oxygen (red) from [25]

\subsection{Electron Loss Processes}

Processes that reduce the number of free electrons, such as attachment, are considered direct loss processes. There are also indirect loss processes that do not reduce the number of electrons, but rather reduce the energy of the free electrons to a point where they can no longer ionize the surrounding molecules. As these processes prevent the generation of free electrons they are still considered loss processes. In addition, the lower energy of the electrons can also aid in the direct loss processes, such as attachment, as they tend to have greater effects at lower energies. 


\subsubsection{Electron Attachment}

The first direct path through which free electrons are lost is the process of attachment to electronegative gases, such as oxygen and $\mathrm{SF}_{6}$. Because these molecules have outer electron shells that are not full, they will attract and scavenge the free electrons. This process occurs primarily at lower energies and is aided by the excitational collisions discussed below. The collision cross sections for attachment by oxygen molecules can be seen in Figure 2.4. It should also be noted that nitrogen, a dominant gas in air, has no known stable negative ion.

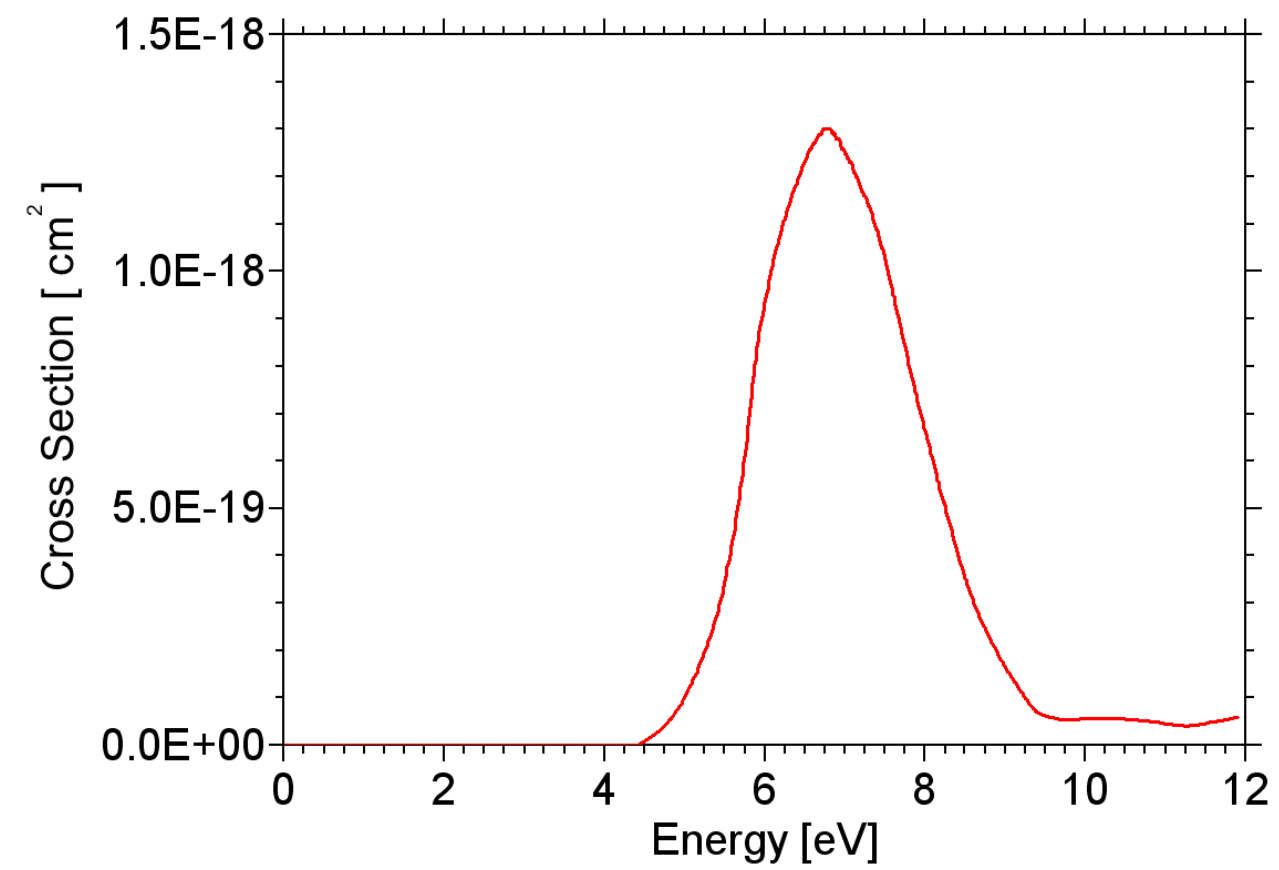

Figure 2.4: Attachment collision cross section for oxygen [26]

\subsubsection{Diffusion}

Diffusion is the primary process by which electrons are lost to the volume. This occurs primarily through the natural spreading of electrons given their random motion 
and frequent collisions. Even when the electrons are under external excitation, such as the applied field, diffusion still occurs in a superimposed manner due to the frequent collisions. The diffusion constant, $D$, is given in equation (2.4) with $\bar{v}$ as the average speed of the electron and $\bar{\lambda}$ as the mean free path [16].

$$
D=\frac{\bar{\lambda} \cdot \bar{v}}{3}
$$

\subsubsection{Recombination}

Another way the number of free electrons is reduced is through recombination. This occurs because ions and electrons that are close together will tend to recombine. Two common ways for recombination to occur are described below.

$$
\begin{aligned}
& e+A_{2}^{+} \rightarrow A^{*}+A \quad \text { (Electron Ion Recombination) } \\
& e+A^{+} \rightarrow A^{*}+h v \quad \text { (Radiative Recombination) }
\end{aligned}
$$

In radiative recombination an excited molecule $\left(\mathrm{A}^{*}\right)$ and a photon $(h v)$ are produced. It is possible for the photon to ionize another molecule, and the excited molecule is more likely to be ionized by an electron impact. The rate of recombination can be determined from the recombination coefficient, $\beta$, which is on the order of $10^{-7} \mathrm{~cm}^{3} / \mathrm{s}$ at temperatures ranging from room to several $\mathrm{kK}$ [17]. The characteristic decay time can then be calculated from $\tau_{\mathrm{r}}=\left(\beta n_{e}^{o}\right)^{-1}$, with $n_{e}^{o}$ being the initial electron concentration. Recombination is not used in the Monte Carlo simulation, since the electron and ion concentrations are low for the time scales considered. 


\subsubsection{Collision Losses}

The most common type of collision is an elastic collision in which an electron is deflected by the larger molecule. Given the electrons mass as compared to that of the molecules $(\sim 1: 51000)$ the amount of momentum transferred in the collision is negligible. Collision cross-sections for both oxygen and nitrogen molecules are show in Figure 2.5.

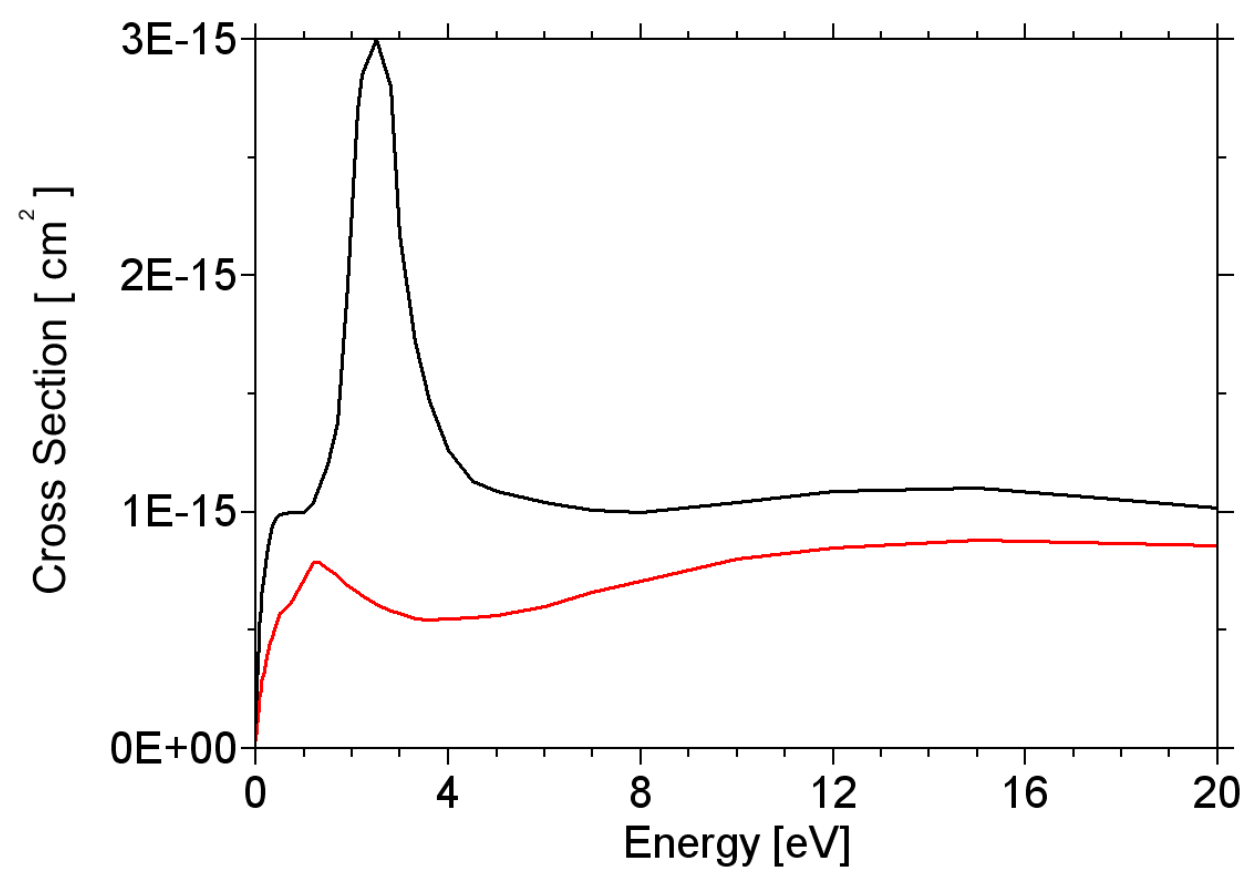

Figure 2.5: Elastic collision cross sections for nitrogen (black) and oxygen (red) from [27] and [28], respectively

\subsubsection{Excitation Losses}

Excitational collisions result in the excitation of the molecule without ionizing it. These collisions are grouped into three categories: vibrational, rotational, and electronic excitation. The incident electron loses the amount of energy required for the change of quantum states. For instance, in the nitrogen molecule, $0.29 \mathrm{eV}$ is required to go from the ground state to the first vibrational state $(\mathrm{v}=1)$. All of the vibrational, rotational, and 
electronic collision cross sections used for both oxygen and nitrogen molecules are shown in Figure 2.6 thru Figure 2.11.

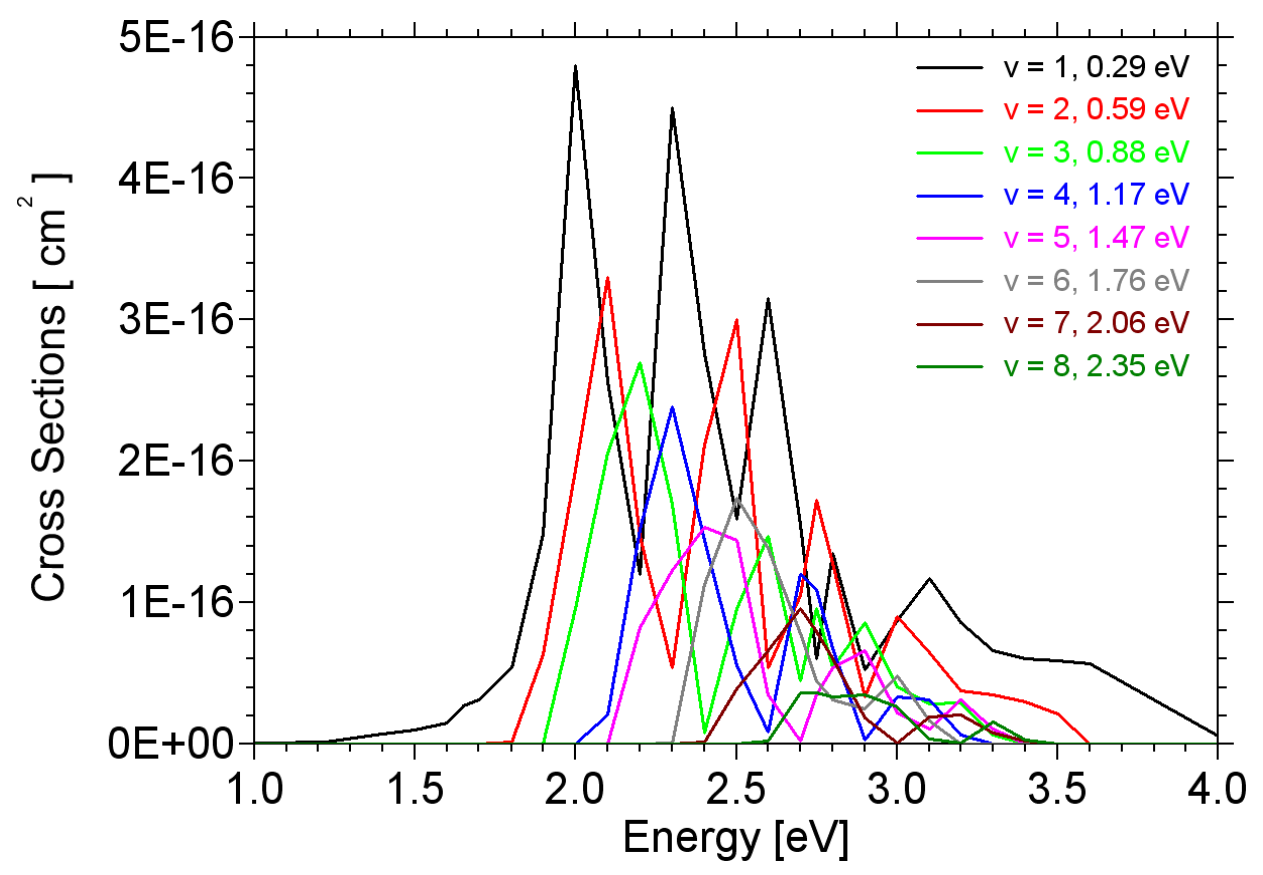

Figure 2.6: Vibrational collision cross sections for nitrogen. The excited vibrational state for the electronic ground state along with the energy lost by the impacting electron is shown. [27] 


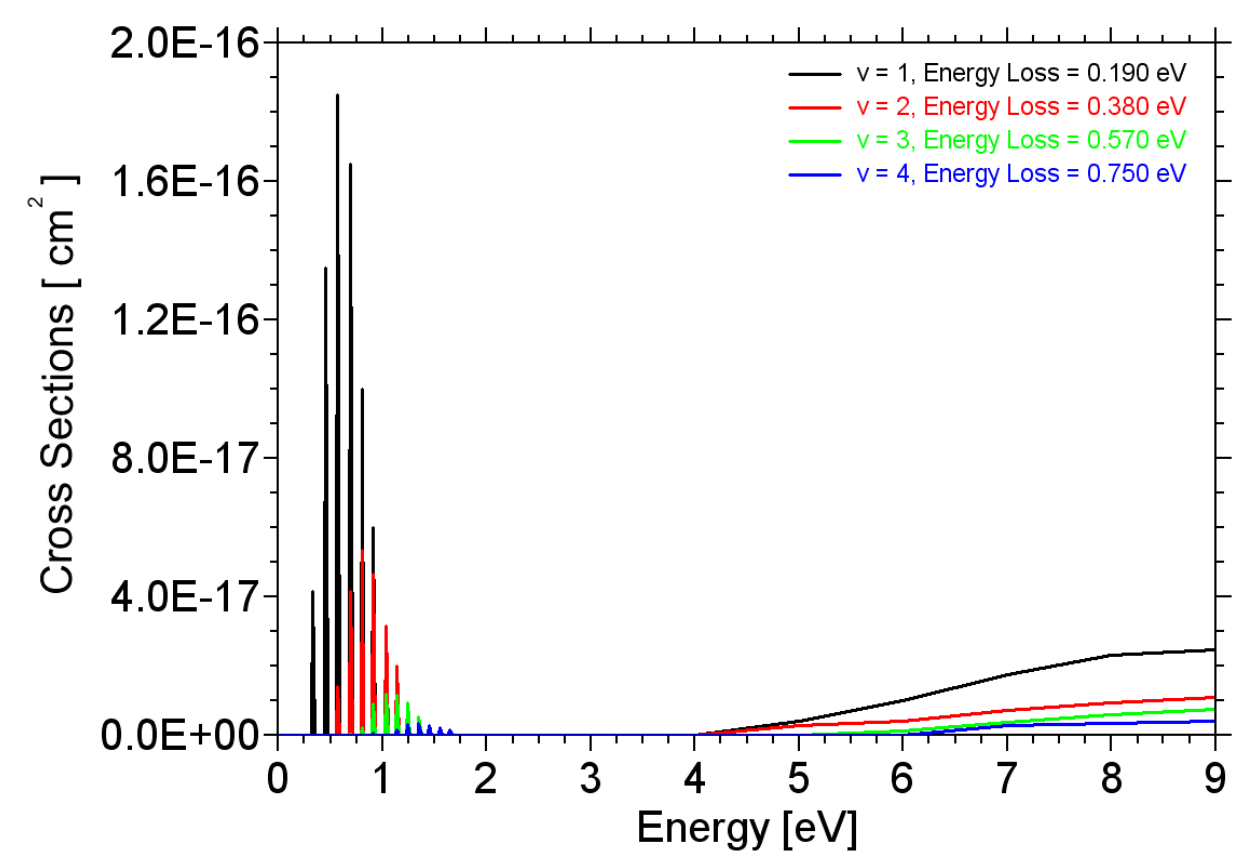

Figure 2.7: Vibrational collision cross sections for oxygen. The excited vibrational state along with the energy lost by the impacting electron is shown. [28]

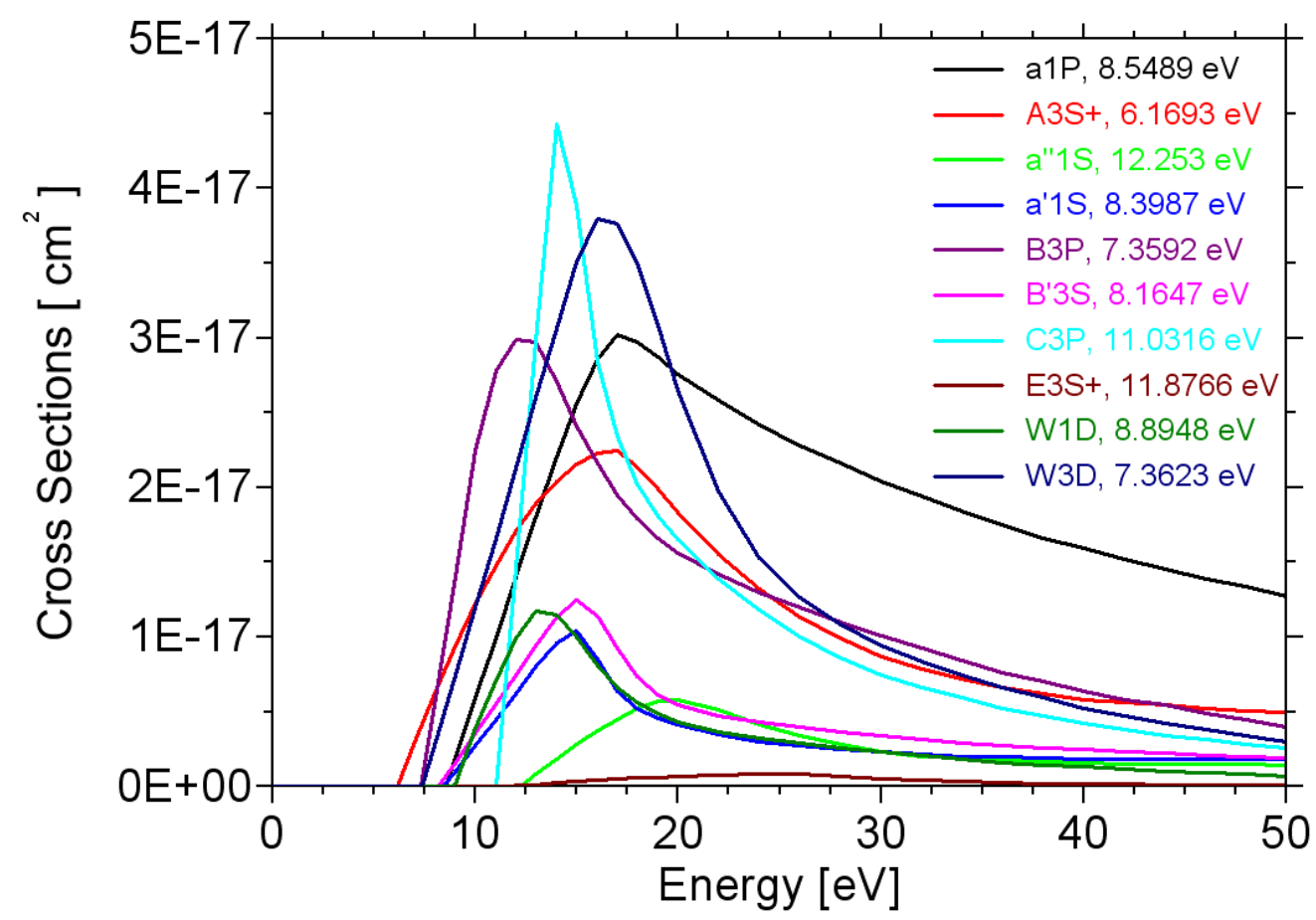

Figure 2.8: Electronic collision cross sections for nitrogen. The electronic transition as well as the energy lost by the impacting electron is shown. [29] 


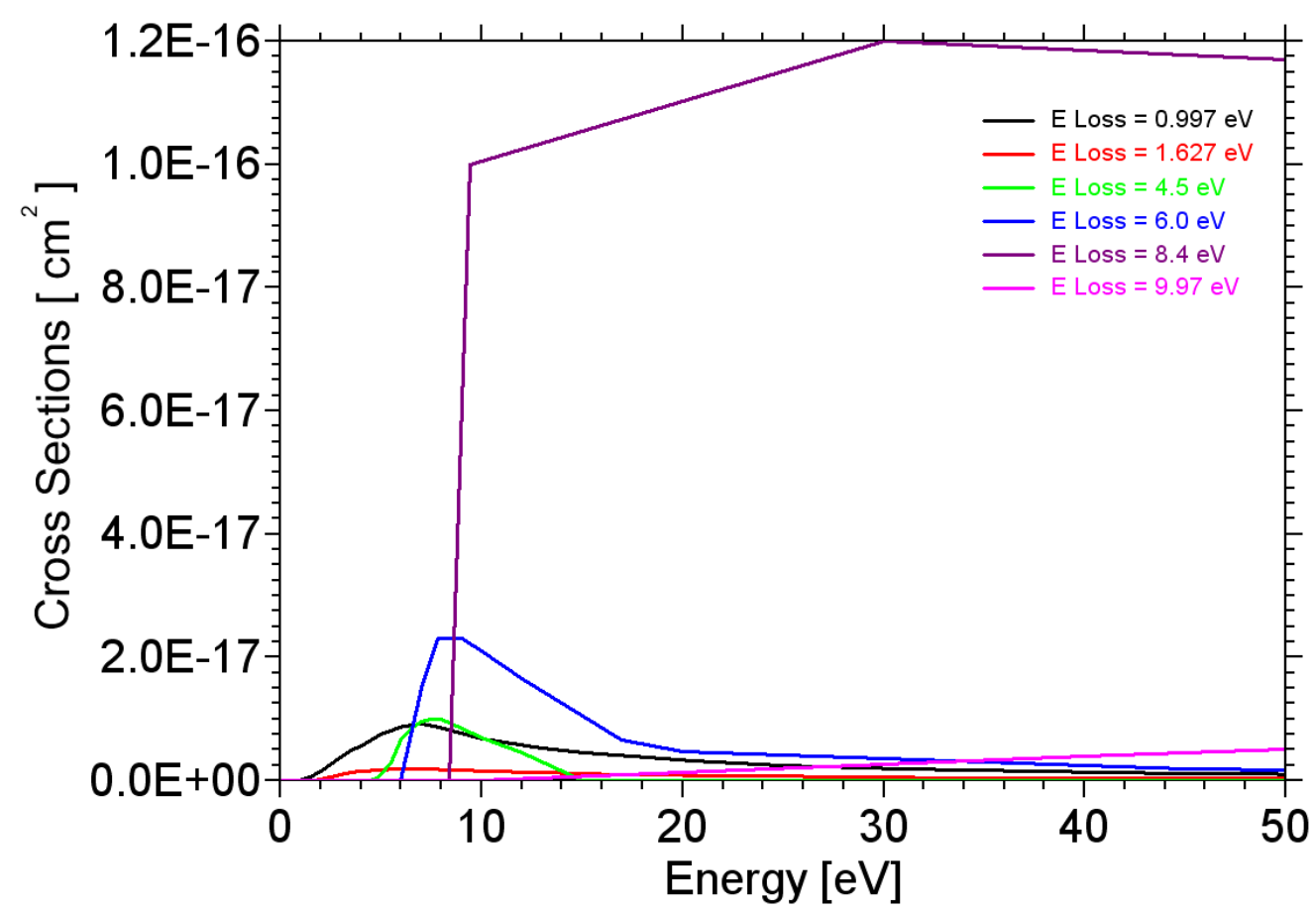

Figure 2.9: Electronic collision cross sections for oxygen. The energy lost by the impacting electron is shown. [28]

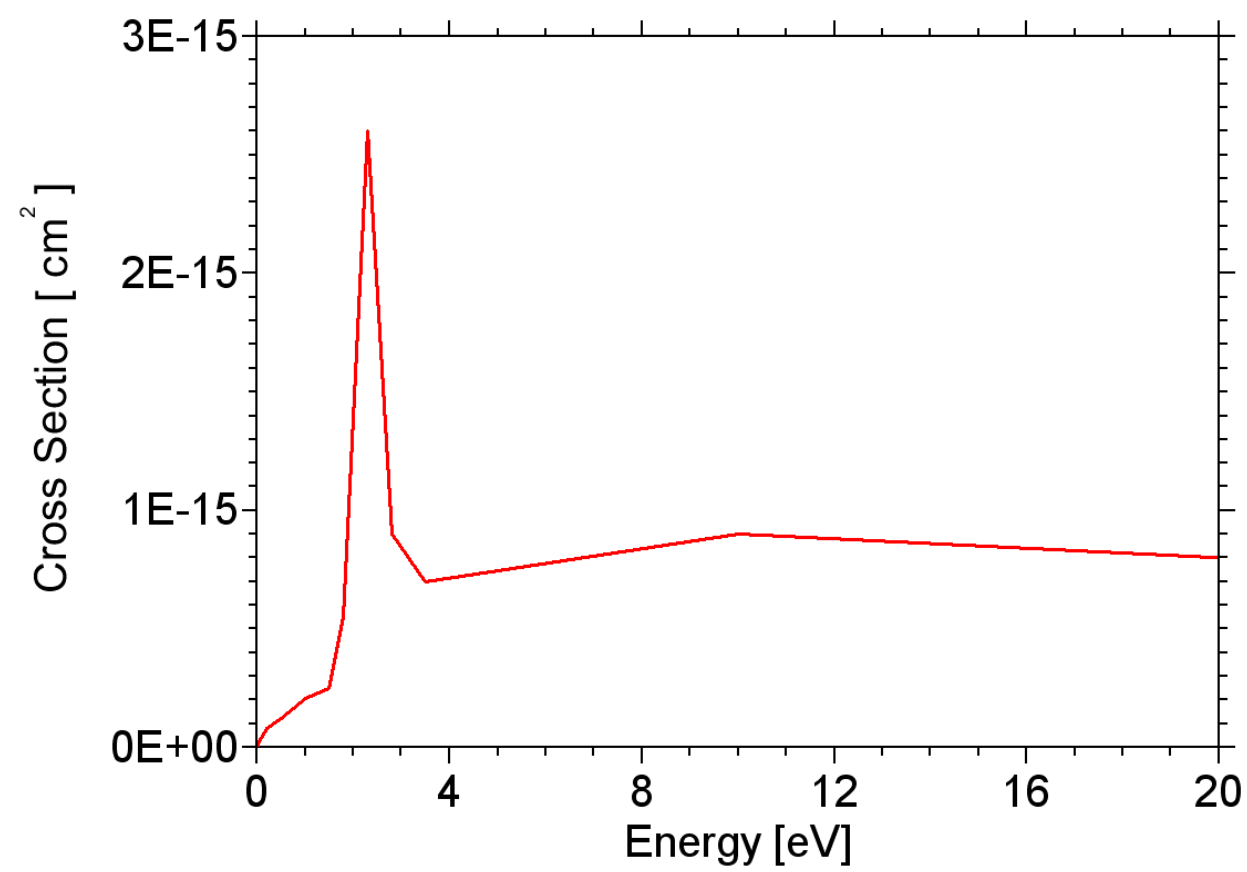

Figure 2.10: Rotational collision cross sections for nitrogen. [30] 


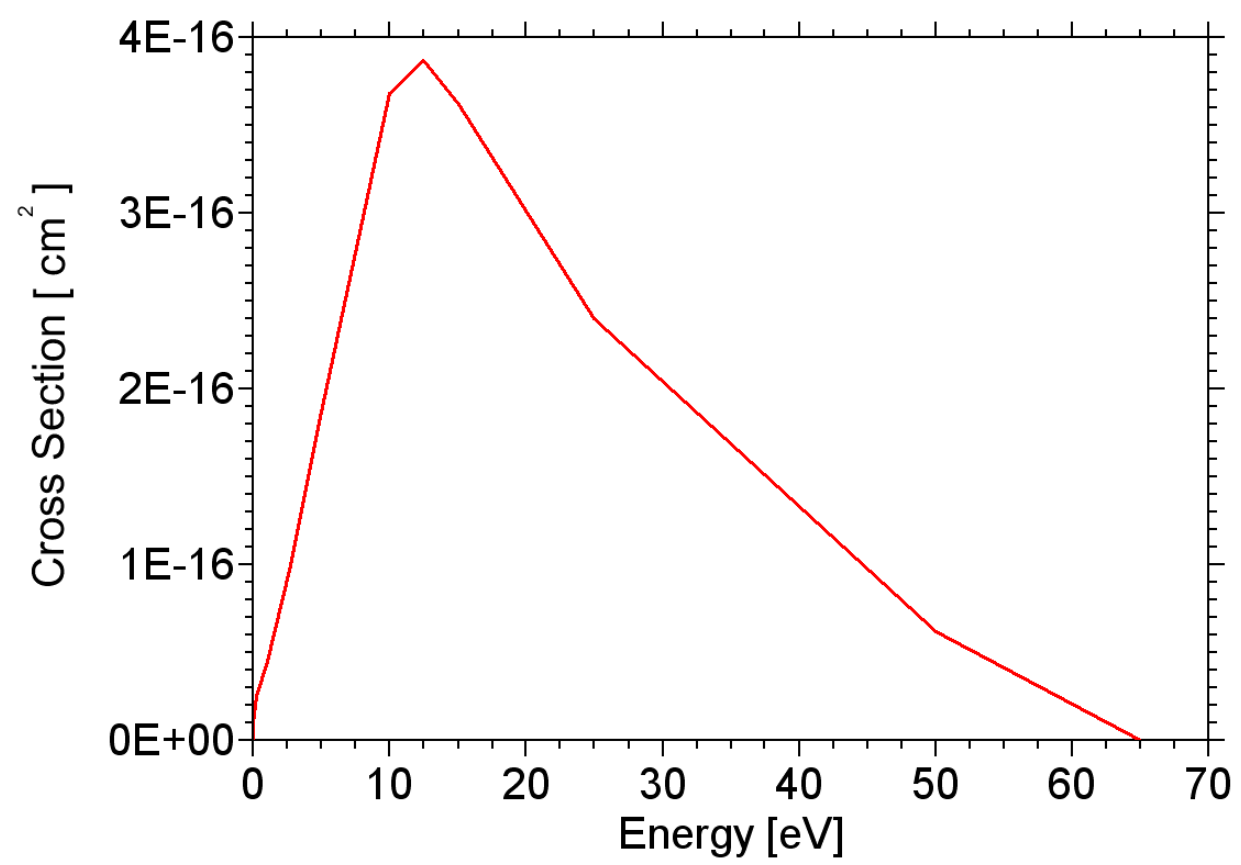

Figure 2.11: Rotational collision cross sections for oxygen. [31]

\subsection{Summary of Collision Cross Sections}

Several differences exist in the various cross sections for both nitrogen and oxygen. For ionization the cross sections have very similar overall shapes; however, important differences appear at lower energies. The ionization of oxygen begins at $\sim 12.5 \mathrm{eV}$ as opposed to $\sim 16.5 \mathrm{eV}$ for nitrogen. As a result, oxygen will ionize more readily than nitrogen and have a greater impact on the initial formation of the breakdown. In the case of attachment, oxygen plays a large role as a strongly electronegative gas while there is no known attachment associated with nitrogen. The scavenging of free electrons by oxygen provides an important loss mechanism not present in a pure nitrogen environment. Nitrogen is clearly dominant in elastic collisions, which are the most frequent type of collisions as evident by the large overall values of the cross sections. The 
vibrational cross sections share a similar multi-peak shape at lower energies but the oxygen cross sections, unlike the nitrogen, contain contributions at energies above $4 \mathrm{eV}$. The contribution of oxygen at higher energy levels decreases the availability of electrons with sufficient energy for ionization. Electronic excitation collisions are similar in both nitrogen and oxygen, with oxygen exciting at a lower initial energy than nitrogen. Finally, nitrogen clearly dominates oxygen for rotational collisions, as evident by the increase magnitude of the nitrogen cross section.

\subsection{Volume Breakdown Mechanisms}

Breakdown occurs when the current through the gap becomes self-sustaining and the potential across the gap collapses. Two groups of processes control the formation of the breakdown, the electron amplification processes and the electron loss processes. In volume breakdown, without the presence of a dielectric surface, the direct electron loss processes taken together form the loss coefficient, $\eta$, the average number of electrons lost per unit length, while the amplification processes form the ionization coefficient, $\alpha$. The amplification and loss processes compete, one increases while the other decreases the total number of electrons. A new value called the effective ionization coefficient, $\alpha_{e f f}$, is defined as $\alpha-\eta$. In order for a net ionization to occur $\alpha>\eta$ must be satisfied. If a dielectric surface is present, the integral treatment of loss and amplification has to be abandoned and localized processes have to be added. There exist many comprehensive literature sources describing these processes in volume breakdown, including books by 
Nasser, Raizer, Brown and Papoular [16, 17, 32, 33], as such, only a brief treatment will be given here.

\subsubsection{Townsend Mechanism}

J. S. Townsend began investigating the V-I curve for two parallel plates in the early 1900s $[34,35]$. Specifically, he looked at the region beyond saturation, i.e. between $V_{2}$ and $\mathrm{V}_{4}$ in Figure 2.12. In the region beyond saturation ionization of the gas begins and results in electron multiplication. The equation describing the growth of electron population, $n$, as a function of distance from the cathode, $d$, initial electrons, $n_{o}$, and the effective ionization coefficient, $\alpha_{\text {eff }}=(\alpha-\eta)$, is shown below:

$$
n=n_{o} \cdot e^{\alpha_{e f f} \cdot d}
$$

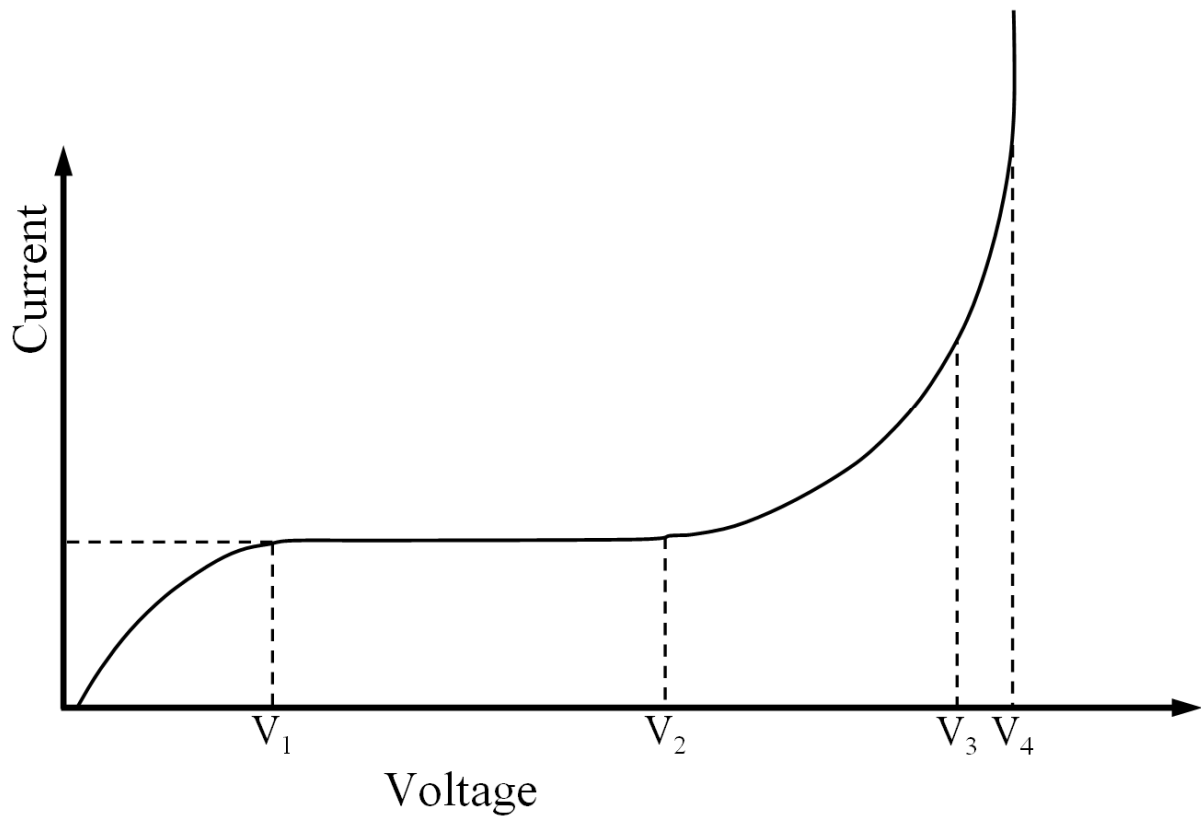

Figure 2.12: Townsend V-I curve for gap current vs. potential [36] 
The number of electrons will increase exponentially provided that $\alpha_{\text {eff }}=\alpha-\eta>1$. The growth of the electrons occurs in the opposite direction of the applied electric field and is called an electron avalanche. Figure 2.13 shows the distribution of ions and electrons within the avalanche. As the avalanche moves forward, the head grows radially outward due to diffusion and the electrostatic repulsion of the newly formed electrons. The electrons move much faster than the ions and, in the time scale considered, the ions are effectively stationary. A result of the difference in speed is that the electrons occupy the head of the avalanche while the ions are left behind and compose the cone shaped tail.

It should also be noted that in addition to electron multiplication, electron avalanches also produce photons. This is due to the fact that during the building of the avalanche, gas atoms are both excited and ionized. Some of the excited states have very short lifetimes and will return to their ground state, emitting photons as they do, before the avalanche is complete. This will play an important role in streamer formation discussed later.

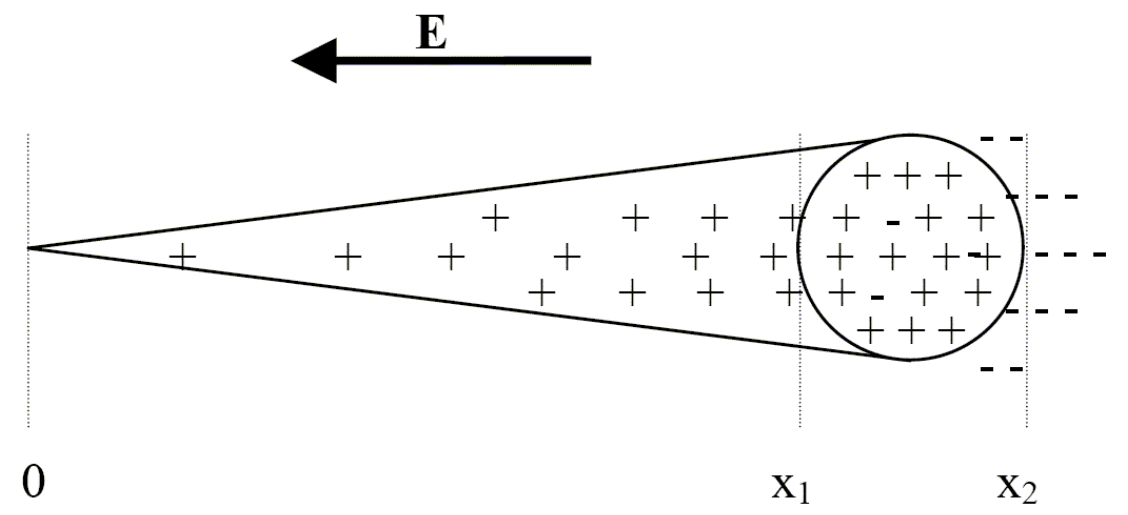

Figure 2.13: Diagram of electrons and ions in an electron avalanche, showing ion $(+)$ and electron (-) distributions [36] 
In order to achieve a self sustained discharge a process must exist that provides more seed electrons from the cathode. The coefficient of secondary electron emission, $\gamma$, includes the effects these processes, such as positive-ion bombardment of the cathode and bombardment of the cathode by photons generated by the excited gas. Now that the electron amplification is in place along with a means of continually providing seed electron to be amplified, a criterion for self-sustaining discharge can be shown, as in equation (2.6).

$$
1-\gamma \cdot e^{\alpha_{e f f} \cdot d} \geq 0
$$

\subsubsection{Streamer Formation}

In higher fields, the electron avalanche alone often cannot describe the transition from electron emission to breakdown. There is first an avalanche-streamer transition which then leads to a streamer-breakdown transition. Streamers, named for their filamentary nature, are the combination of multiple electron avalanches. 


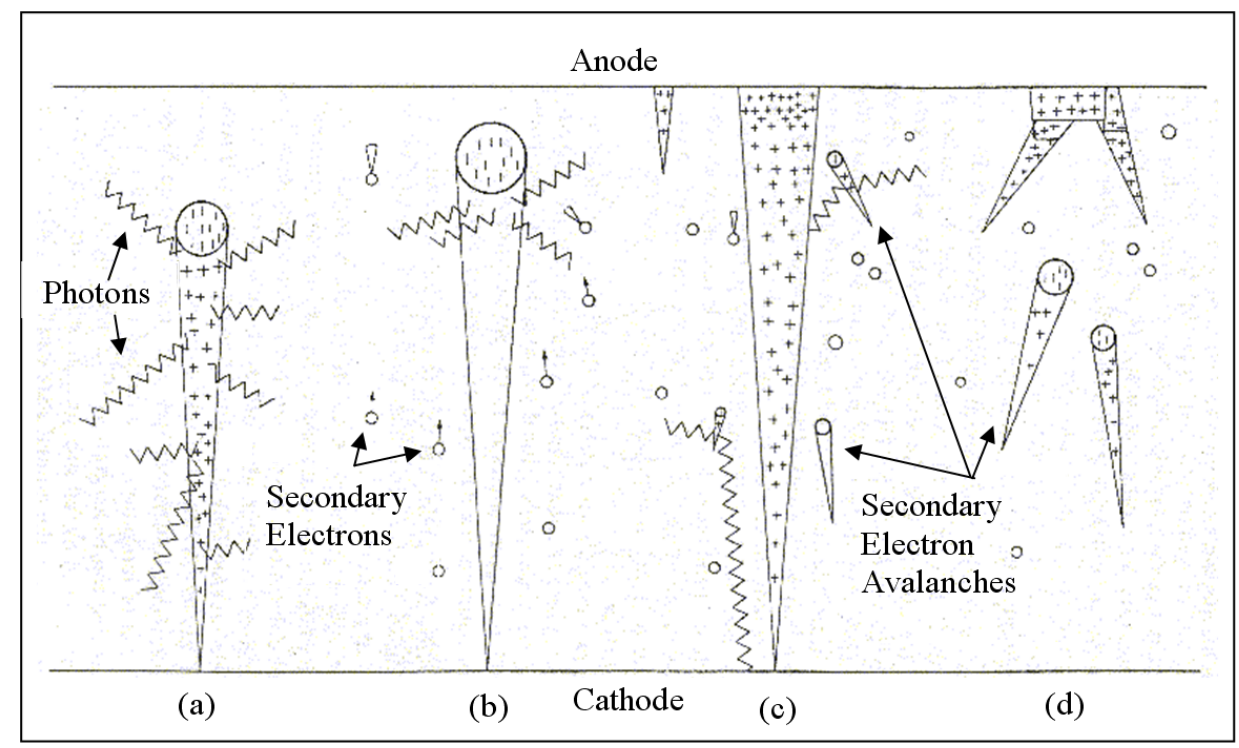

Figure 2.14: Diagram of early stages in the streamer development, showing primary and secondary electron avalanches, space charges caused by ions and electrons, as well as the role of photoemission [17]

Figure 2.14 describes the process of streamer formation [17]. As the primary avalanche forms, photons are emitted in all directions and are absorbed at various distances away from the avalanche head as in Figure 2.14(a). The photons in turn can ionize and excite other gas molecules, thereby creating more free electrons, as in Figure 2.14(b). Because of the strong space charge created by the ions and electrons in the avalanche, the field near the avalanche approaches the same order of magnitude as the background electric field. This field enhancement increases the effective ionization constant and aids the secondary electron avalanches initiated by the newly created photoelectrons. The secondary avalanches also emit photons and along with electrons from the initial avalanche create third generation avalanches and so on. The enhanced field also causes any later generation avalanches to grow towards the main branch. When the primary avalanche reaches the anode the electrons are absorbed and a layer of ions is 
left in close proximity to the anode channel with the same total charge as the head of the avalanche as in Figure 2.14(c). This layer of ions forms the beginning of the ionization channel. Second generation avalanches are attracted to the field enhancement caused by the ionization change, causing them to grow toward the main trunk. When the secondary avalanches come in contact with the primary ionization channel the electrons are drawn to the anode via a charge gradient in the channel, while the ions contribute to the head of the ionization channel. Because the secondary avalanches are initiated at several different points, the ionization channel takes on a filamentary shape as seen in Figure 2.15(e). Some filaments continue to grow while others stall because of a lack of impacting avalanches needed to continue their development. Eventually, the streamer will bridge the gap between the electrodes, as in Figure 2.15(g), and the ionization channel becomes the breakdown path. The transition from Townsend avalanche to streamer formation is difficult to quantize. In the Townsend model the space charge of the ions left behind by the avalanche is ignored. Since the space charge plays an important role in the streamer formation the point at which the space charge is on the order of the applied electric field is determined by the cross-over point from a Townsend to a streamer discharge. Raether's breakdown criterion shows that this transition occurs when approximately $10^{8}$ electrons are present in the head of the electron avalanche [37, 38]. Streamer formation, while relevant for unipolar excitation, is not considered the best theory for RF breakdown, particularly for the current parameter ranges. This is due primarily to the fact that the oscillating field prevents electrons from moving across the gap, resulting in relatively stationary electron cloud growth. 


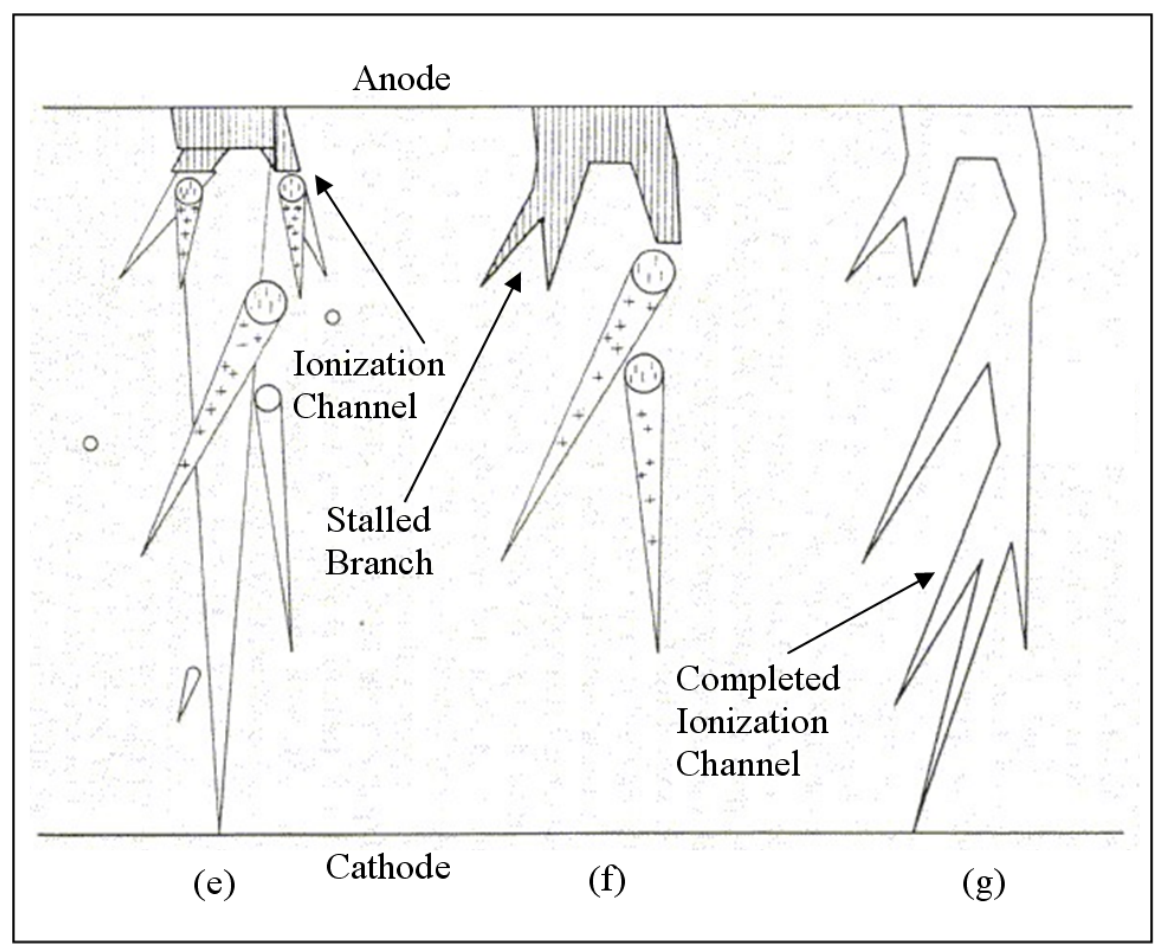

Figure 2.15: Diagram of late stages in the streamer development, including the development of the ionization channel through continued electron avalanche additions and the final completed channel

\subsubsection{Secondary Electron Emission Avalanche}

Secondary Electron Emission Avalanches, or SEEA's, are one of the most widely accepted mechanisms that contribute to surface flashover in vacuum [2]. The process begins when electrons, usually emitted from the cathode at the triple point, impact the surface and produce secondary electrons as outlined previously. In the process of liberating electrons from the surface, the surface is left with a net positive charge, causing the electrons to be drawn back to the surface. Some of the secondary electrons then impact the surface again creating two more free electrons. The continuation of this process leads to a SEEA illustrated in Figure 2.16. 


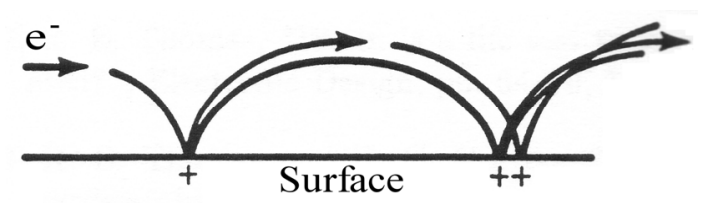

Figure 2.16: Diagram of secondary electron emission avalanche [2]

\subsection{Previous Research of Unipolar Flashover}

Several theories exist as to the mechanisms of surface flashover under unipolar excitation, most of which utilized the idea of secondary electron emission. However, it is important to note that these theories were developed for surface flashover in vacuum. After discussing some of the processes responsible for surface flashover in vacuum the applicability of these processes in an air environment at atmospheric pressure will be discussed.

The breakdown can be divided into three stages: (1) initiation, (2) development, and (3) final stage. It is generally agreed that the electron emission from the triple point is the initiating event in surface flashover. It is also generally agreed that the final stage discharge develops in a layer of desorbed gas from the surface [2]. The processes involved in the development stage are the subject of a range of differing theories. Most of the work done in this project, and in general, is involved with the development stage.

Anderson and Brainard, noting that a surface of a ceramic in vacuum is covered with a layer of adsorbed gas, postulated that the SEEA bombardment of the surface desorbed some of the gas [39]. The desorbed gas is then partially ionized, creating a positive space charge near the cathode. This space charge, along with a positive surface charge due to SEE, increases the field strength at the cathode, which then increases the field 
enhancement at the triple point. This enhanced field causes increased electron emission, which in turn desorbs more gas to be ionized, creating a regenerative amplification process that quickly leads to breakdown. It is also shown by Neuber, that through Townsend-like activity in the desorbed gas layer, a positive space charge is formed in the gap near the surface. The field enhancement caused by the space charge creates enhanced field emissions from the cathode [40].

The primary difference between surface flashover in air and vacuum is the amount of molecules in the volume. In vacuum electrons experience few if any collision with background gas molecules on their way from cathode to anode. As a result, some of the electrons impact the anode, and the surface, with significant energies on the order of the applied potential. At higher pressure the number of background molecules increases as does the number of collisions experienced by the electrons. As a result of these collisions the electrons do not gain energy as freely as in vacuum. The energy of the electrons now has a strong dependence on the frequency of the electron-molecule collisions. This is why an arc at higher pressure is considered a collision dominated process.

There are also processes in vacuum that do not occur in an air environment, for example, in vacuum there is a very limited supply of molecules to be ionized, thus necessitating the desorption of gas from the surface to make molecules available to be ionized and form the ionization channel. The presence of large amounts of molecules for ionization in air eliminates the importance of gas desorption in the breakdown process. The air environment affects the breakdown in other ways as well, for instance, in vacuum the mean free path of a field emitted electron is much greater than that of an electron 
emitted in air. This means that field emitted electrons have a smaller chance of impacting the surface before interacting with the molecules in the air. However, electrons that do not make it to the surface contribute to the excitation and ionization of molecules in the air. Through secondary processes discussed in the previous sections, these excited and ionized molecules then produce photons which are also capable of ionizing other molecules or liberating electrons from the surface. Finally, in air, as opposed to vacuum, the arc is not confined to developing in a thin layer of desorbed gas. This means that, given the right conditions, the arc can form a significant distance away from the surface.

In an air environment, the flashover is still believed to contain three stages; however, the processes present in each are different than in vacuum. The initiation stage remains basically unchanged with the initial electrons being emitted from the triple point due to field enhancements. The development stage involves the combined processes of electron impact ionization, photo-ionization, UV induced electron emission, SEE across the surface with possible negative gain, and Townsend amplification which all contribute to flashover development. These processes are discussed in detail for volume breakdown in air by Pedersen [41]. However, it is not known what roles these processes play in surface flashover or to what extent each is involved. The final stage involves the streamer ionization channel bridging the gap which completes the breakdown.

\subsection{Previous Research of HPM Breakdown}

The Townsend discharge criterion, while originally developed for DC discharges, has proven valuable for discharges produced at any frequency, including those in the 
microwave range [42]. In the classic Townsend model, breakdown is defined in terms of ionization processes versus loss processes. In general, if the number of the electrons produced by ionization events is equal to the number lost due to processes including diffusion, recombination and attachment, then a steady state discharge will occur. In reality such a steady state discharge is difficult to generate without current limiting by the external drive circuit. If the production rate of electrons and ions is slightly larger than the loss rate, the gas quickly becomes ionized and breakdown occurs.

The applicability of the Townsend ionization processes with electric fields in the microwave frequency range was first proven by Herlin and Brown [43]. The electric fields generated by RF excitation exert an oscillatory force on the free electrons such that very little distance is traversed in one-half cycle. This oscillatory nature, for frequencies above $500 \mathrm{MHz}$, limits the loss mechanisms to diffusion, attachment, and recombination as opposed to the electrons being "swept" out of the gap by a unipolar field.

A detailed theory of volume breakdown in air under pulsed RF excitation can be found in literature sources [42, 44, and 45]. Gould and Roberts [44] and MacDonald [42] were the first to develop models for microwave breakdown. Pulsed breakdown fields are higher than those of CW fields, and are dependent on both the pulse length and the rate of repetition. Breakdown takes place for pulsed conditions if the electron concentration reaches the critical plasma density by the end of a given pulse $[45,46]$. The critical plasma density, $n_{c}$, is defined in equation (2.7), where $\omega$ is the angular frequency of the electromagnetic field, $q_{e}$ is the charge of an electron, $\mathrm{m}_{\mathrm{e}}$ is the mass of an electron, and $\varepsilon_{\mathrm{o}}$ is the permittivity of free space. 


$$
n_{c}=\frac{\omega^{2}}{q_{e}{ }^{2}} \cdot \varepsilon_{o} \cdot m_{e}
$$

The plasma density is equal to $2.4 * 10^{11} \mathrm{~cm}^{-3}$ and $3.6^{*} 10^{14} \mathrm{~cm}^{-3}$ for $2.85 \mathrm{GHz}$ and 110 $\mathrm{GHz}$, respectively. 


\section{CHAPTER 3}

\section{EXPERIMENTAL SETUP}

Two separate experimental setups are used for testing under RF and unipolar excitations. It is well know that in order to observe the dynamics of surface flashover, a temporal resolution in the nanosecond time regime is required. Hence, the test apparatus are carefully designed to ensure that the shape of the transient signals is due to the flashover itself and not distorted by the external electrical circuit. The placement of diagnostics must also be carefully considered, for example the inputs of the spectrograph must be placed very close to the sample to prevent the air from absorbing any UV radiation. Finally, the environment around the flashover surface gap itself must be controllable.

\subsection{Unipolar Testing Apparatus}

In the unipolar case the transient properties of the external excitation setup are an important design consideration. For this reason the entire setup from power supplies, charging lines, and the chamber itself are coaxial and have matched impedances. A complete description of the setup is given by Krile [47]. An overall view of the test setup is shown in Figure 3.1. 


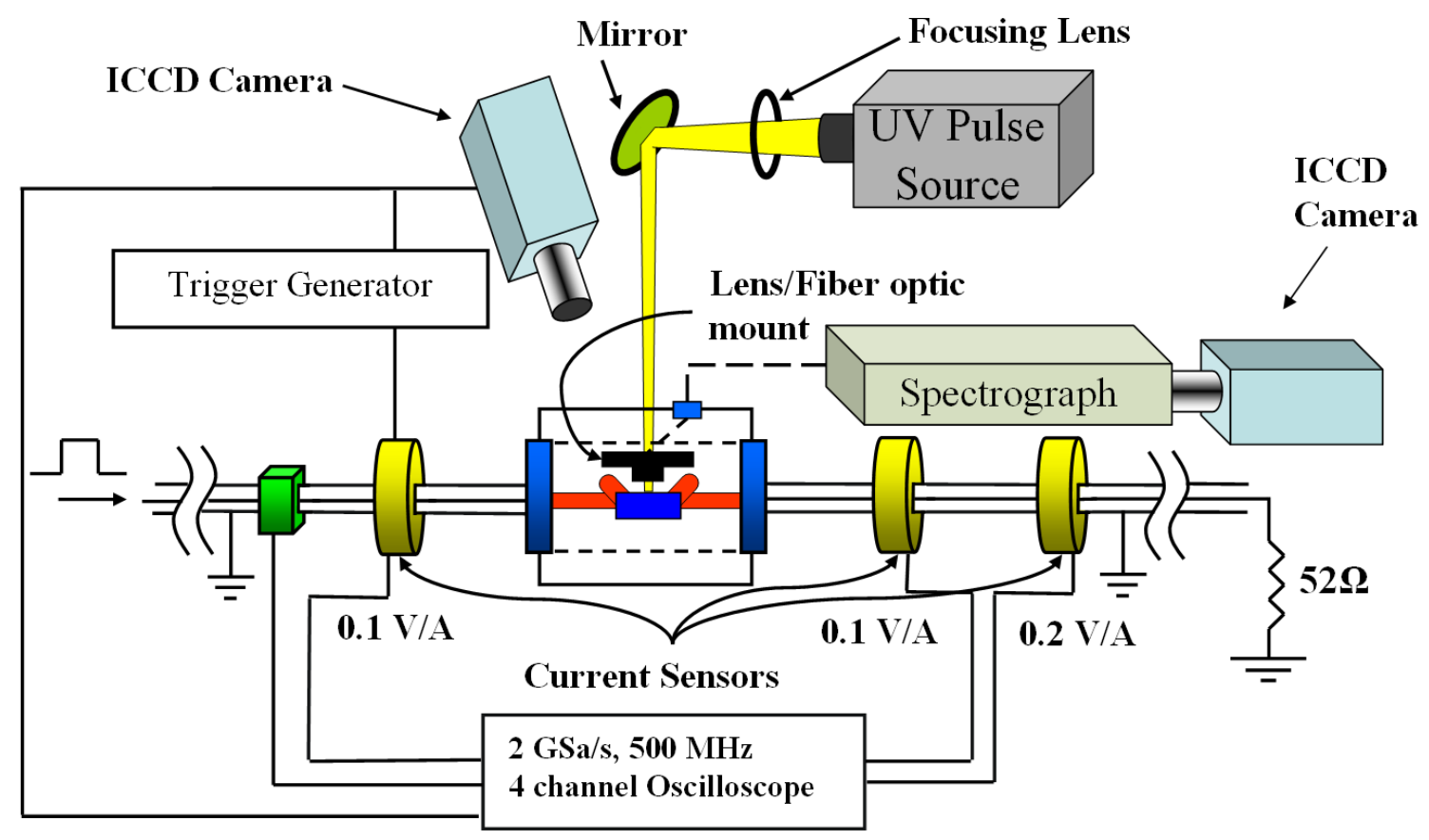

Figure 3.1: Overview of entire unipolar setup shown for pulsed excitation. For DC excitation the lines are charged to equal and opposite voltages. Environmental controls are omitted

\subsubsection{Test Chamber}

The testing chamber is designed with visibility, accessibility, and control in mind. The chamber is 8 inches wide, 6 inches long and 7 inches tall. Constructing the chamber of clear polycarbonate allows for the placing of high speed cameras and other diagnostic tools in any location to get the best view of the flashover. The lid provides easy access to the interior of the chamber and seals the chamber so that the application of a small positive pressure to the inside of the chamber will ensure an isolated controllable environment.

A cross section of the test chamber with lid, charging line feedthroughs, and hemispherical electrodes is shown in Figure 3.2. Finally, a picture of the test chamber 
including fiber optic and charging line feedthroughs, current sensors, camera, and pressure vent valve can be seen in Figure 3.3.

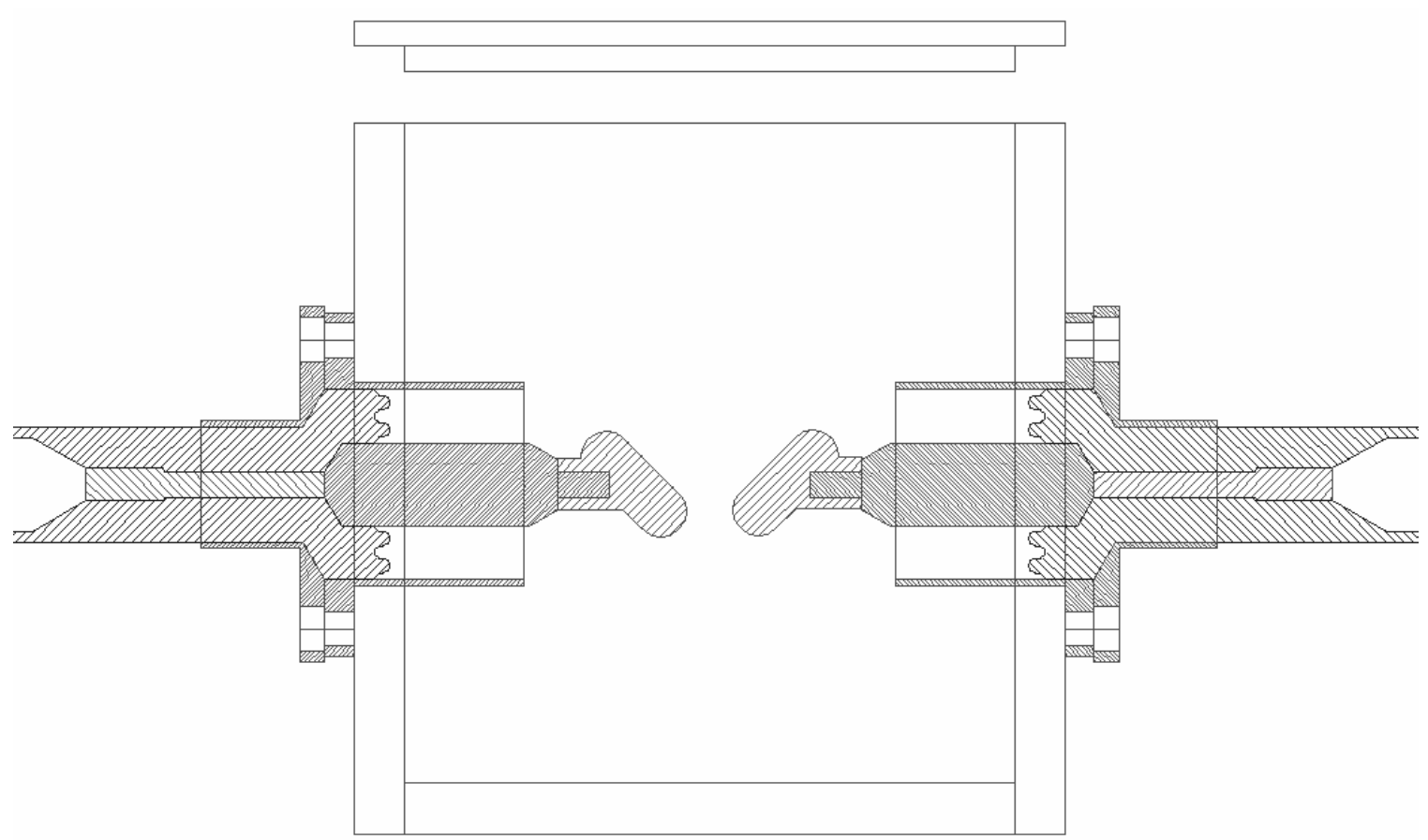

Figure 3.2: AutoCad drawing of testing chamber with feedthroughs and hemispherical electrodes, removable mesh outer conductor not shown [47] 


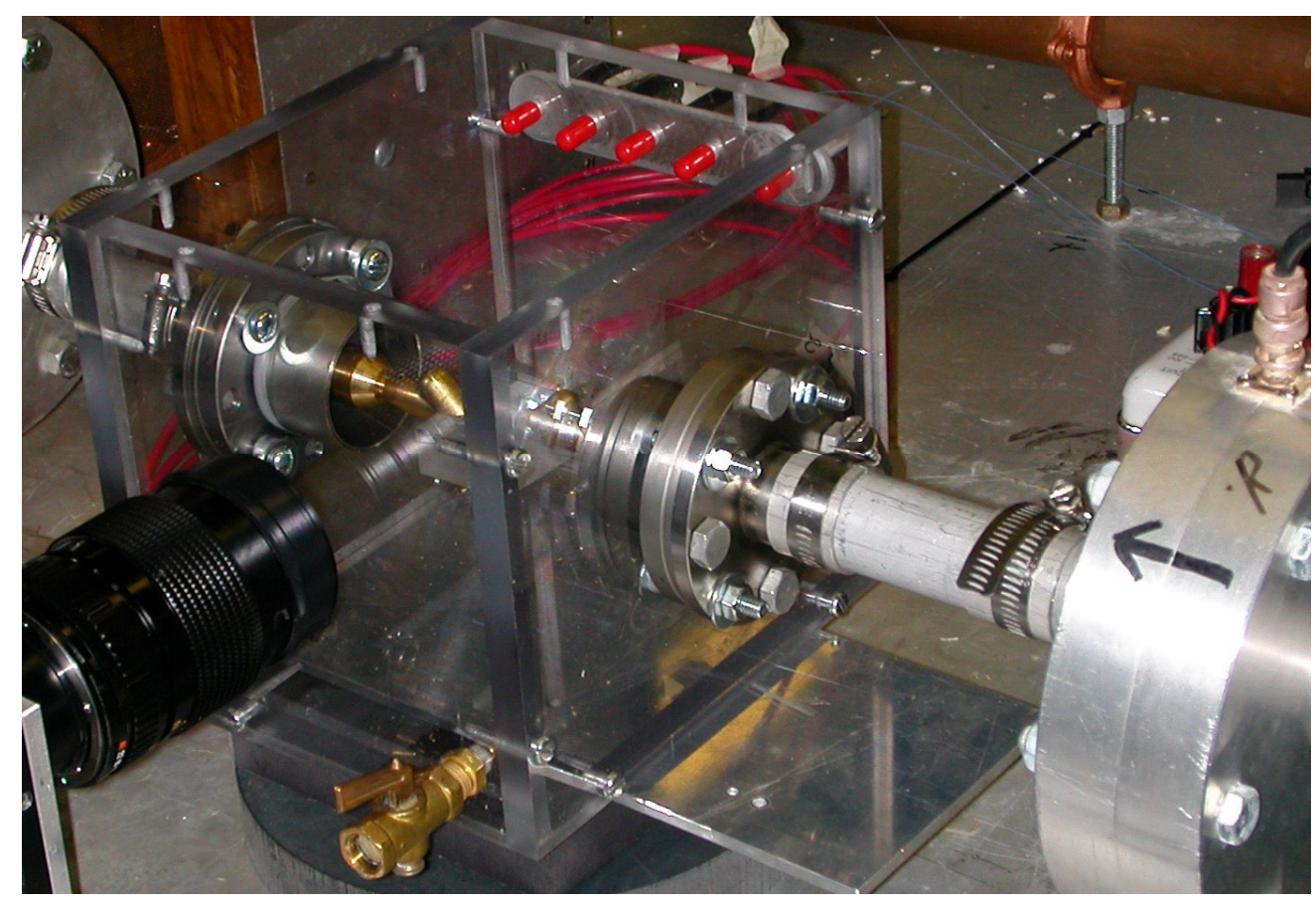

Figure 3.3: Picture of testing chamber with the feedthroughs, current sensors, and camera lens shown

In order to ensure that the entire system, consisting of the charging lines and breakdown gap, has $52 \Omega$ impedance throughout, the cable to gap transitions or feedthroughs become very important. The feedthroughs are mounted to the sides of the test chamber and connect the charging cables outside to the breakdown gap inside. The feedthroughs must both maintain the impedance of the system, but also be gas tight to help maintain the positive pressure inside the chamber. In addition, they must prevent flashovers from occurring across the surface between the inner and outer conductor at the transition between the insulator of the cables and the gas insulator inside the chamber. The ratio of inner and outer conductor must be adjusted to maintain $52 \Omega$ impedance with air as the insulator. 


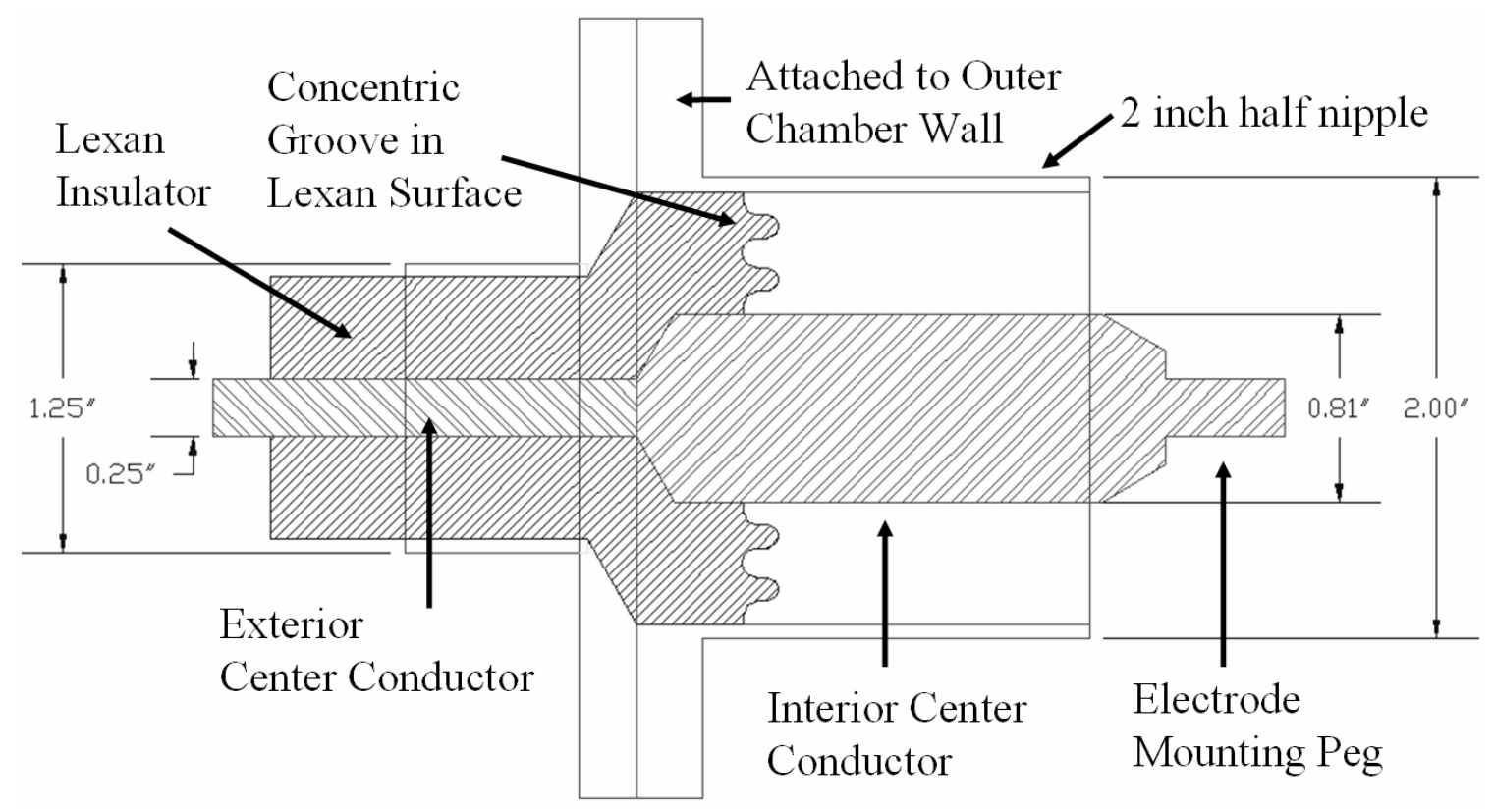

Figure 3.4: Cross-section of transition, also called the feedthrough, from charging to test chamber with key component identification [47]

As can be seen in Figure 3.4, where the polycarbonate insulator terminates, the surface is grooved. This increases the surface path distance in order to prevent surface flashovers from the charged center conductor to the outer ground. The distance between the electrodes is also kept small to prevent unwanted flashover from occurring. The fields resulting from the given geometry are calculated using Ansoft Maxwell's 3D field simulation software and can be seen in Figure 3.5. The simulation shows that the electric field at the electrodes and across the gap is much greater than across the grooved surface. 


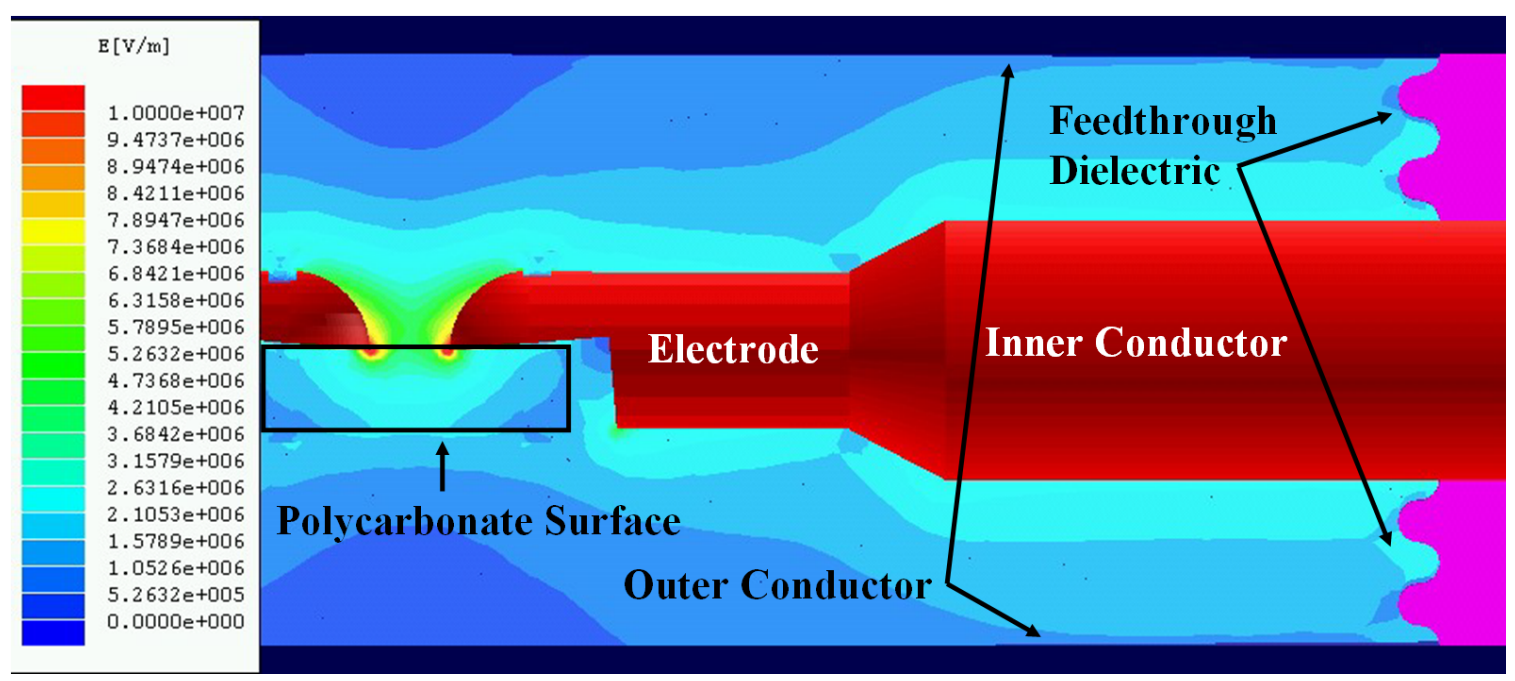

Figure 3.5: Comparison of electric field strengths at electrode tips and at dielectric feedthrough, with each electrode at $\pm 20 \mathrm{kV}$, respectively

\subsubsection{Excitation}

As stated previously, a high temporal resolution in the external charging circuit, including the breakdown test section, is achieved by using a coaxial geometry with a matched impedance of $52 \Omega$ everywhere in the system. For DC charging dual high voltage power supplies are connected to two coaxial cable charging lines connected to either side of the breakdown gap through the chamber feedthroughs.

The dual power supplies, used in the DC case, charge the two charging lines through two $240 \mathrm{M} \Omega$ charging resistors. The power supplies are two Universal Voltronics High Voltage Power Supplies, and are capable of producing up to $140 \mathrm{kV}$ apiece. The two supplies are set to charge to opposite polarities and are connected to the inner conductors of the two charging lines.

For pulsed testing one charging line is terminated into a matched load and the other is replaced with a $0.5 \mathrm{~m}$ length of cable prior to the high voltage pulser. The high voltage 
pulser is custom designed to provide a variable amplitude high voltage pulse. The pulser consists of a high voltage DC power supply connected to a charging capacitor through a $1 \mathrm{M} \Omega$ charging resistor. The capacitor is connected through a $52 \Omega$ matching water resistor to a cylindrical spark gap. The spark gap is triggered by a floating mid-gap center electrode which is pulsed by an external low energy HV-pulser. The gap distance is sufficient to allow for the generation of up to $35 \mathrm{kV}$ pulses with a repeatability of \pm 0.5 $\%$, a rise time of $\sim 20 \mathrm{~ns}$, and a pulse duration of several microseconds. A schematic of the pulser is shown in Figure 3.6 along with an image of the pulser in Figure 3.7.

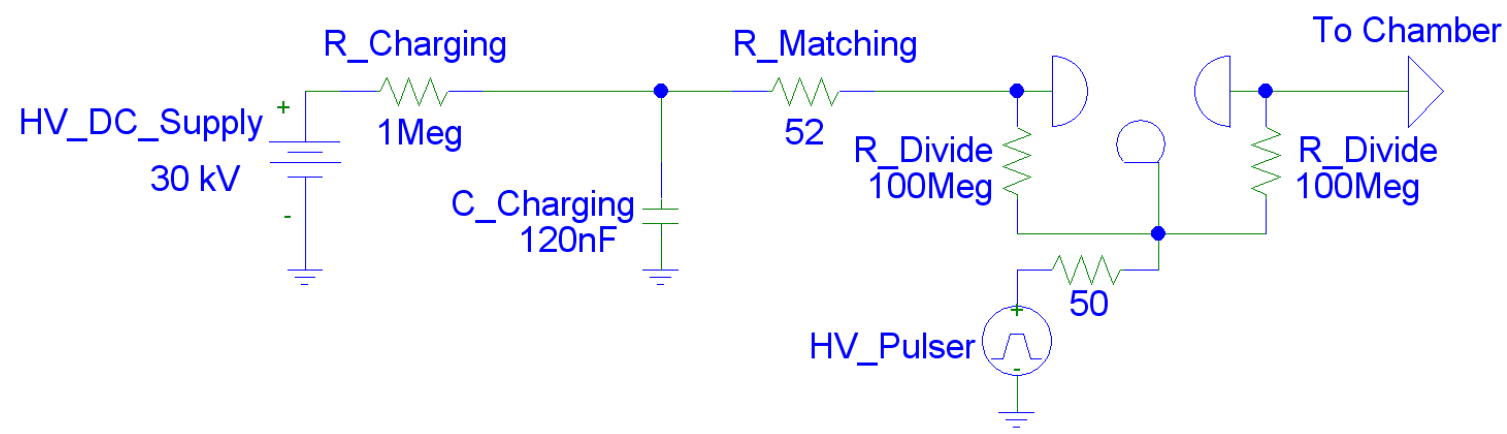

Figure 3.6: Schematic of HV pulser. The center triggering electrode of the spark gap is floated to half the gap potential via the R_Divide resistor 


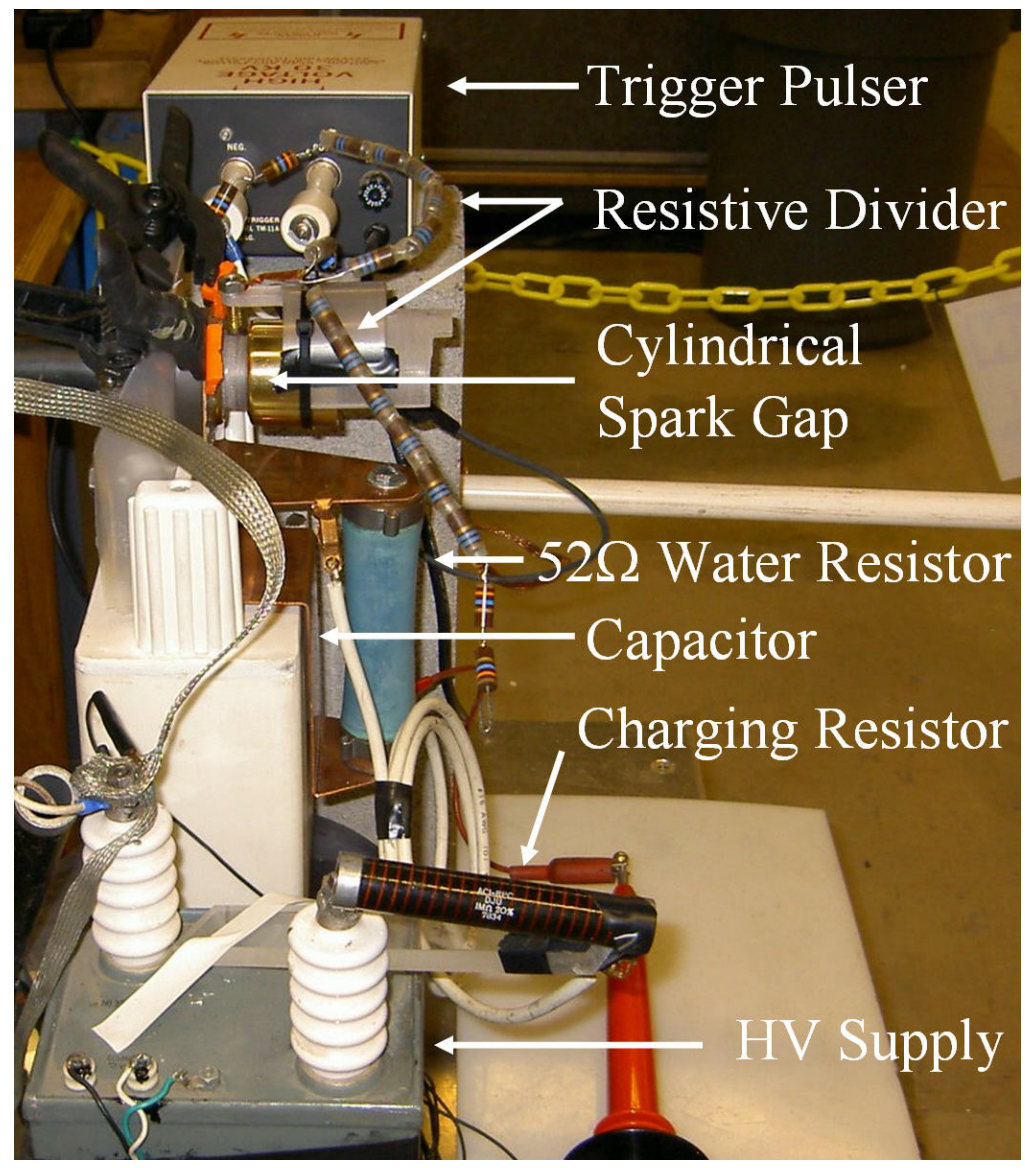

Figure 3.7: Picture of the high voltage pulser setup

The charging lines are made of two 13 meter lengths of RG-220/U coaxial cable with an impedance of $52 \Omega$. The cable is purchased from Times Microwave Systems. The outer conductors are all connected as a common ground. The cables have about a $65 \mathrm{~ns}$ one-way transit time. This time is sufficiently long so that the reflected pulse created by the closing of the gap will arrive back at the gap a significant amount of time, approximately $130 \mathrm{~ns}$, after the breakdown occurs. 


\subsubsection{Environmental Control}

The chamber is designed to allow for certain environmental controls, specifically the relative humidity and type of gas present. The chamber is equipped with a fill line that is used to supply pressurized gases such as nitrogen, argon, or compressed air to the chamber. The gas flow is adjusted to maintain a small constant positive pressure within the chamber.

In addition, a system is in place to regulate the relative humidity within the test chamber. The fill line from the pressurized bottle of gas is split into two lines, each of which is regulated by its own mass flow controller, or MFC, from MKS Instruments Inc. One MFC feeds into an ultrasonic humidifier which is designed to operate in a pressurized system. The output of the other MFC is connected directly to the first mixing chamber along with the output from the humidifier. The output of the first mixing chamber is fed into the bottom of the second. An Omega RH-21C relative humidity and temperature sensor is located inside the second mixing chamber. This sensor has a resolution of $0.1 \% \mathrm{RH}$ and an accuracy of $2.0 \% \mathrm{RH}$. The sensor can measure between $5 \%$ and $95 \%$ relative humidity, and has a 15 second response time. The mixing chambers allow the gas flowing into the test chamber to be maintained at a steady humidity. The humidity is controlled by adjusting the ratio of dry to humidified gas. A schematic of the entire system is shown in Figure 3.8. 


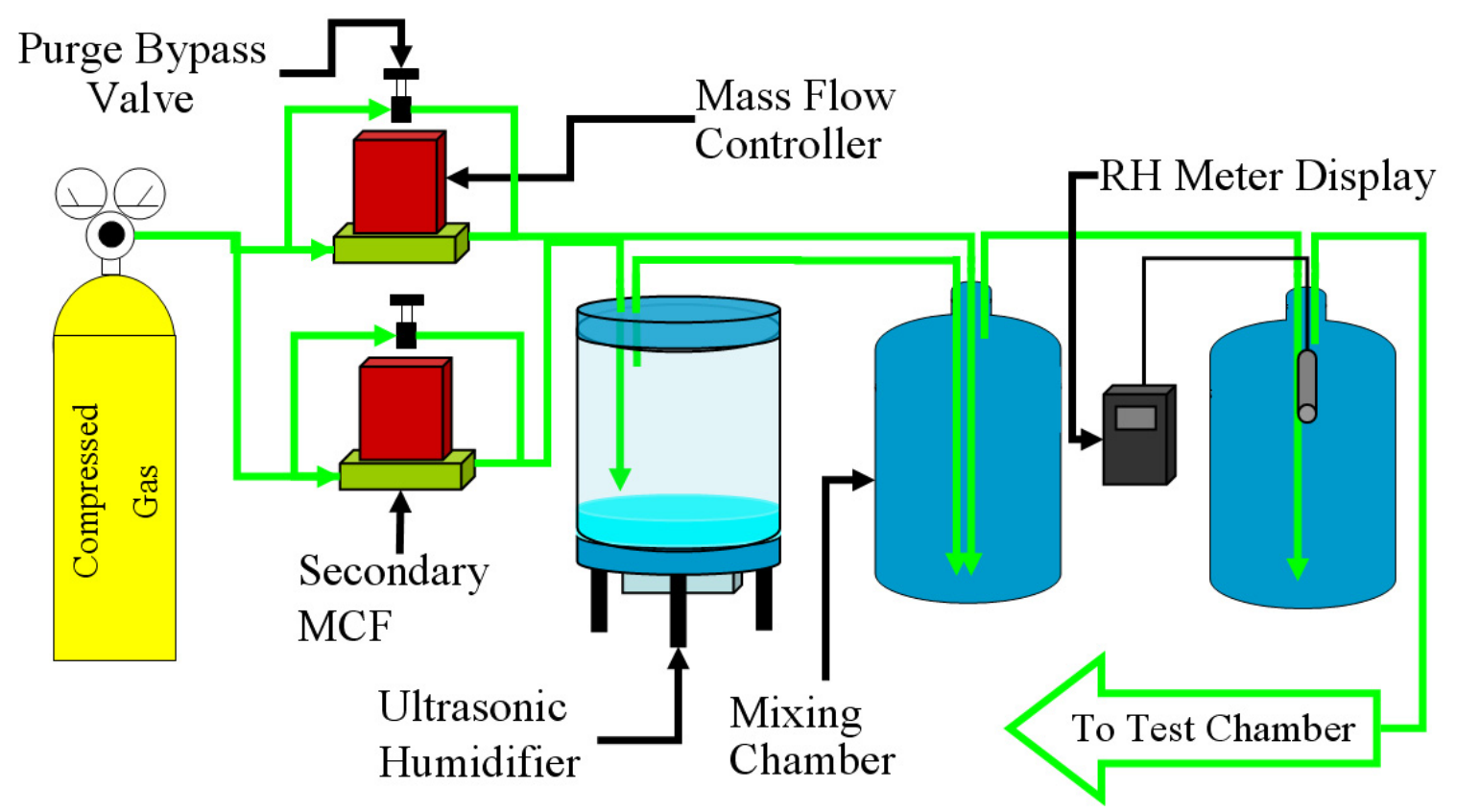

Figure 3.8: Schematic diagram of humidification system. Purge valves are used to force out any humid gas from the system after use

\subsubsection{Electrode Design}

Throughout the course of this experiment, several electrode configurations were utilized. Each design is not necessarily an improvement of the previous design, but better suited for a particular purpose. All of the electrodes are designed to fit on the same mounting pegs, eliminating the need to change the inner conductor inside the chamber. All field simulations were carried out using Maxwell's Ansoft 3D-field simulator software.

\subsubsection{Hemispherical Design}

The hemispherical design is the first design to be implemented. The main idea behind this design was to provide solid contact between the electrodes and the 
polycarbonate surface and to create a relatively predictable arc path. This is accomplished by creating a sphere to sphere configuration in the form of two rounded $1 / 2$ inch brass rods. The spheres are then cut in half with a slight angle to provide a sharp leading edge on the half circle. A hole is drilled in the top of the sphere through which a nylon screw is tightened into a threaded hole in the polycarbonate as shown in Figure 3.9. The tight nylon screws insure that any air gap between the electrodes and dielectric is avoided; as such a gap has a major influence on the hold-off voltage. The electrodes are secured to the mounting peg with set screws.

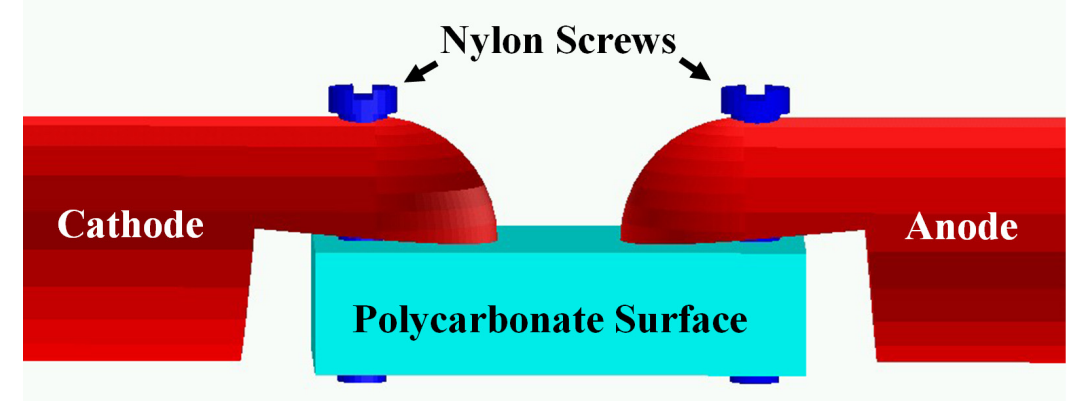

Figure 3.9: Drawing of hemispherical electrodes and attached polycarbonate surface

Several simulations were conducted using Maxwell's Ansoft 3D to determine the electric field strength and direction. As can be seen in Figure 3.10 and Figure 3.11 the simulation shows that the electric field is strongest at the tip of the electrodes, and has a weak normal field component. This configuration is adequate for initial humidity tests and to determine breakdown voltages. This design is also ideal for spectra testing because the arc path is predictable and the light gathering device can be placed with some confidence that the arc path will pass through their region of focus. 


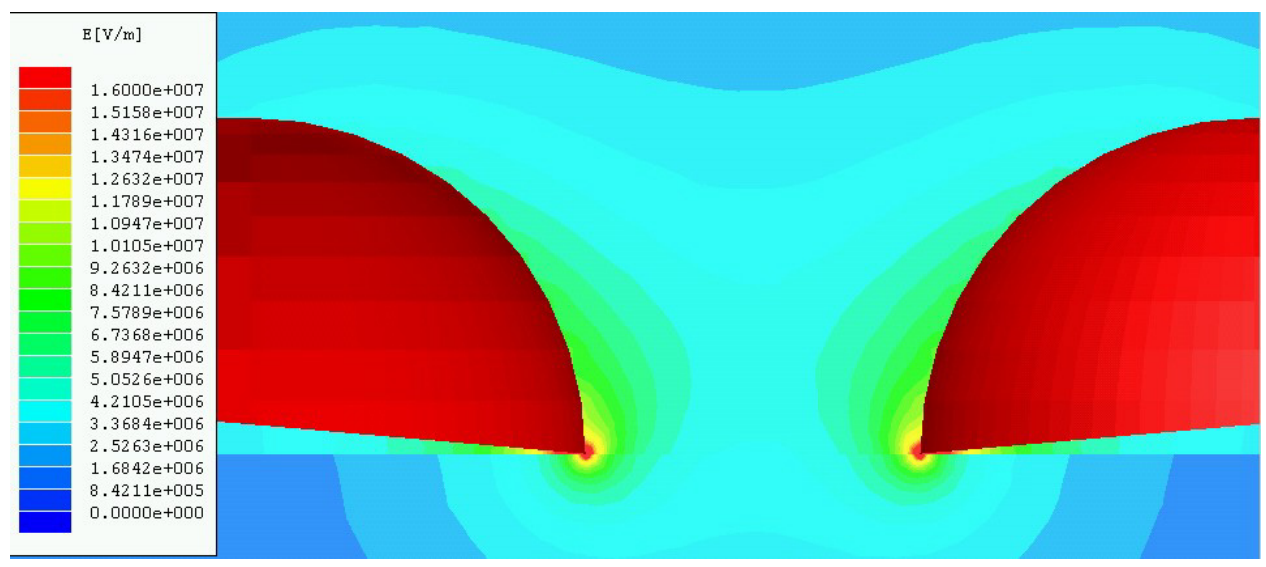

Figure 3.10: Magnitude plot of electric field near the hemispherical electrode tips, gap distance of $6.5 \mathrm{~mm}$, electrodes charged to $\pm 20 \mathrm{kV}$

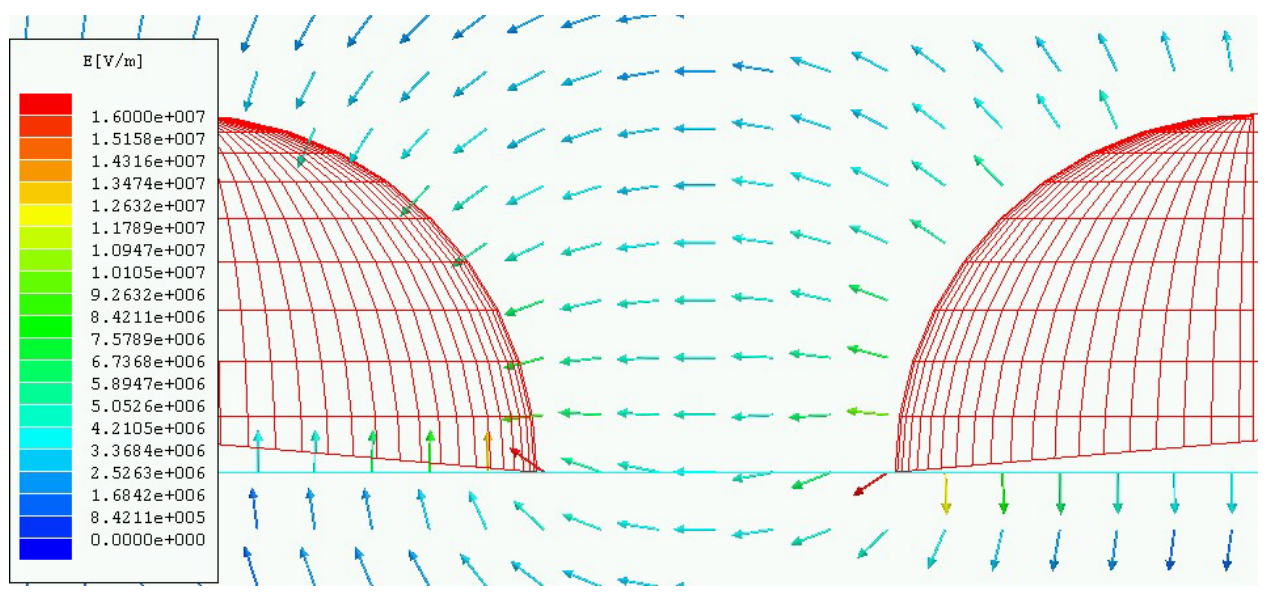

Figure 3.11: Vector plot of electric field near the hemispherical electrode tips, gap distance of $6.5 \mathrm{~mm}$, electrodes charged to $\pm 20 \mathrm{kV}$

\subsubsection{Angled Design}

The next set of electrodes stems from the fact that without a surface present, the arc tends to follow the electric field lines. With the hemispherical electrodes, the electric field lines point into the surface, so it cannot be determined if the arc is trying to follow the field lines or the surface. The angled electrodes are designed to provide an electric 
field with a strong normal component to the surface. To accomplish this, the electrodes are constructed of two 0.5 inch diameter brass rods which are 1.5 inches long with round ends mounted at a $90^{\circ}$ angle from each other as seen in Figure 3.12. The two lower ends of the electrodes are then sunk into two identical diameter holes in the polycarbonate surface. The resulting electric fields and field lines can be seen in Figure 3.13 and Figure 3.14 .

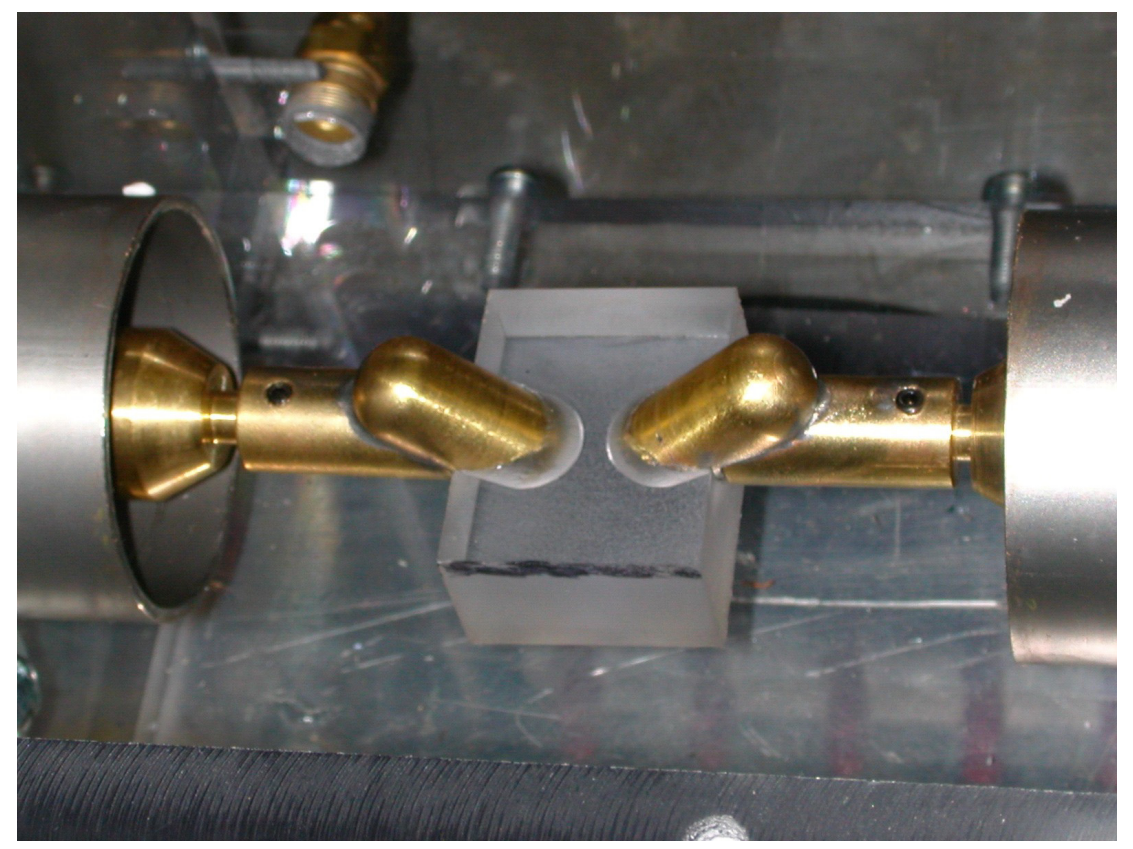

Figure 3.12: Picture of angled electrodes installed in the test chamber with a flat polycarbonate surface 


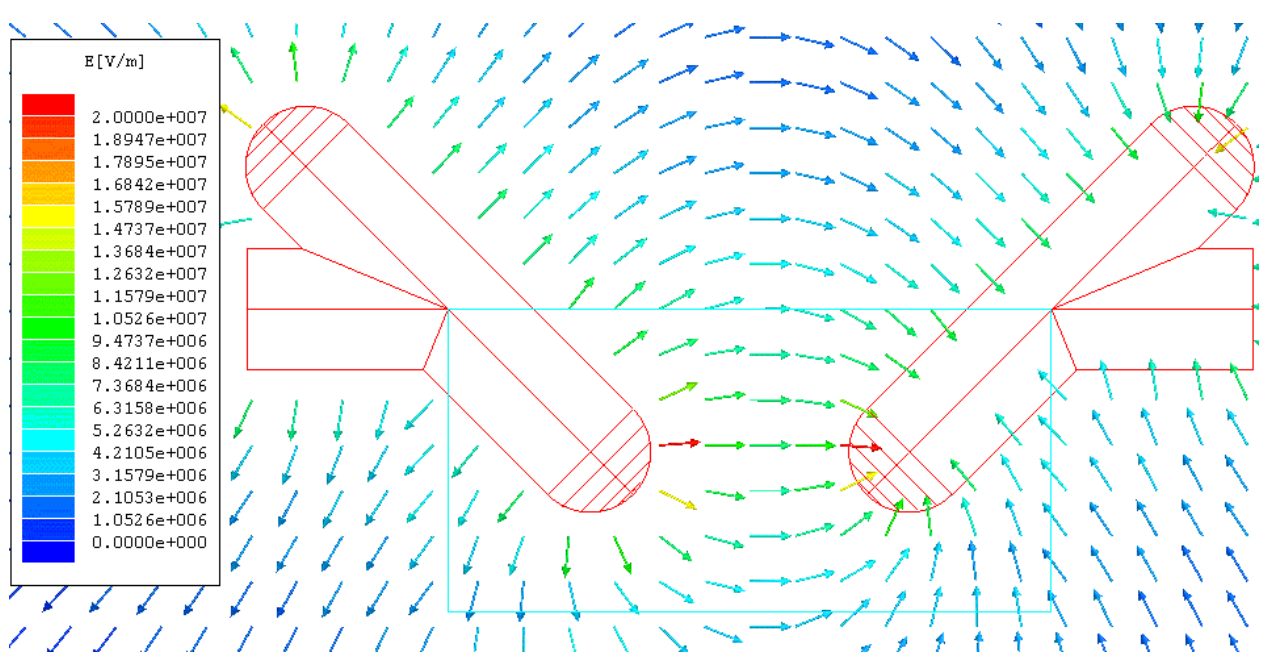

Figure 3.13: Vector plot of the electric field from a side view of the angled electrodes, gap distance of $8.6 \mathrm{~mm}$, electrodes charged to $\pm 20 \mathrm{kV}$

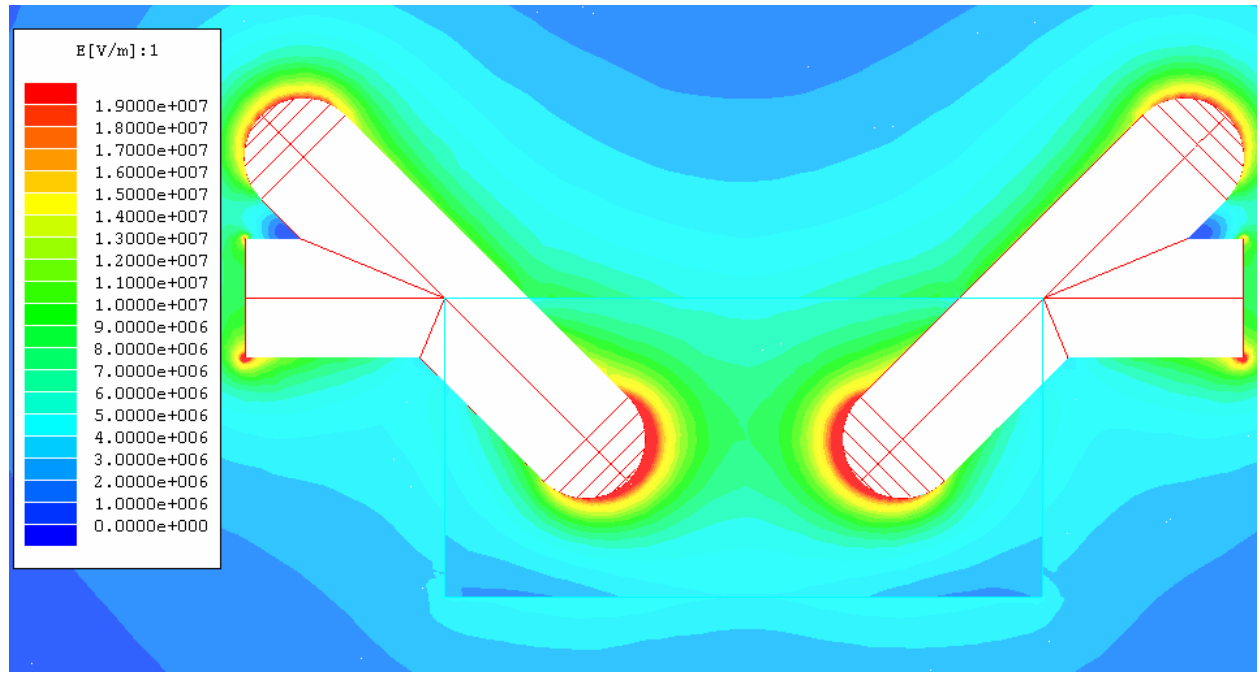

Figure 3.14: Magnitude plot of the electric field from a side view of the angled electrodes, gap distance of $8.6 \mathrm{~mm}$, electrodes charged to $\pm 20 \mathrm{kV}$

While the angled electrodes produce an electric field with a strong component normal to the surface, the path length along the surface is still shorter that the path length along the electric field lines. In order to have more equal path lengths, a groove is cut into the polycarbonate surface. This geometry and the associated field plot are shown below. 


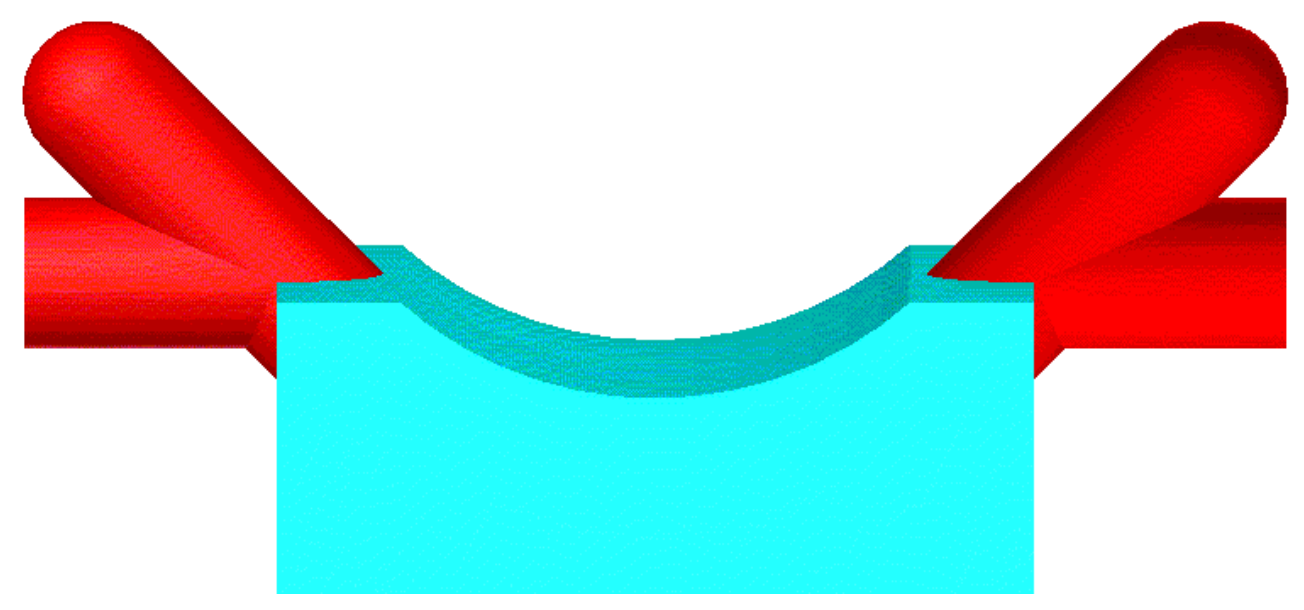

Figure 3.15: Image of angled electrodes inserted into grooved surface

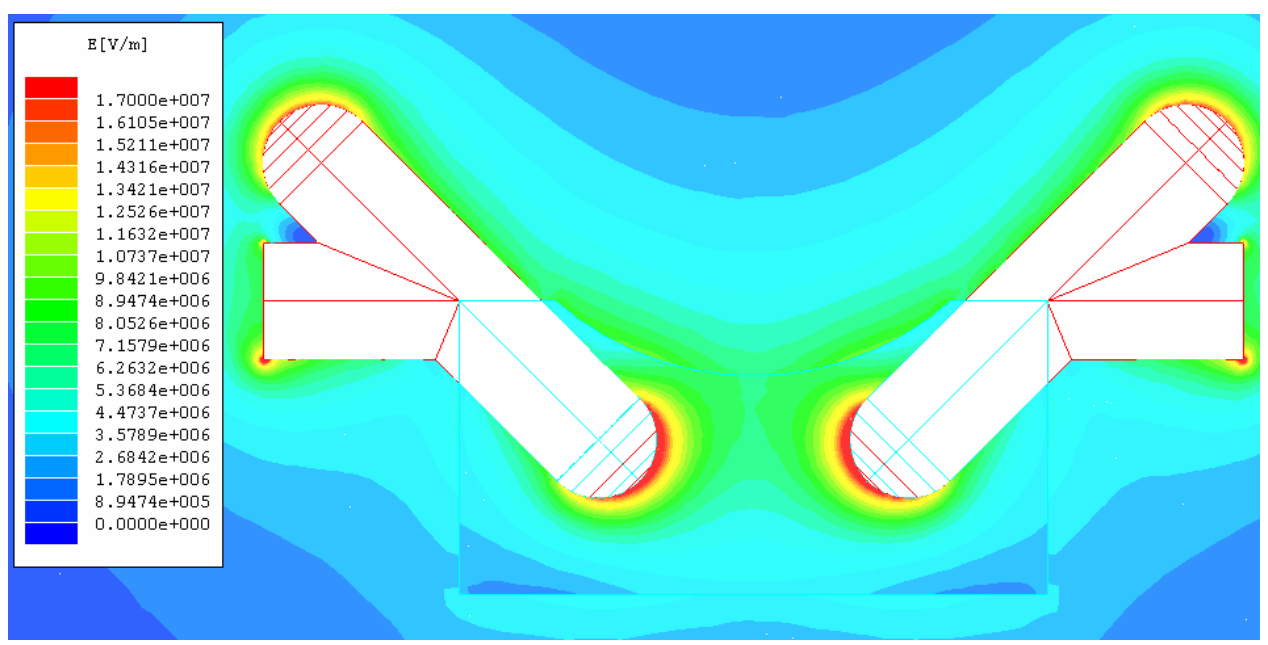

Figure 3.16: Magnitude plot of the electric field from a side view of the angled electrodes with a grooved surface, gap distance of $8.4 \mathrm{~mm}$, electrodes charged to $\pm 20 \mathrm{kV}$ 


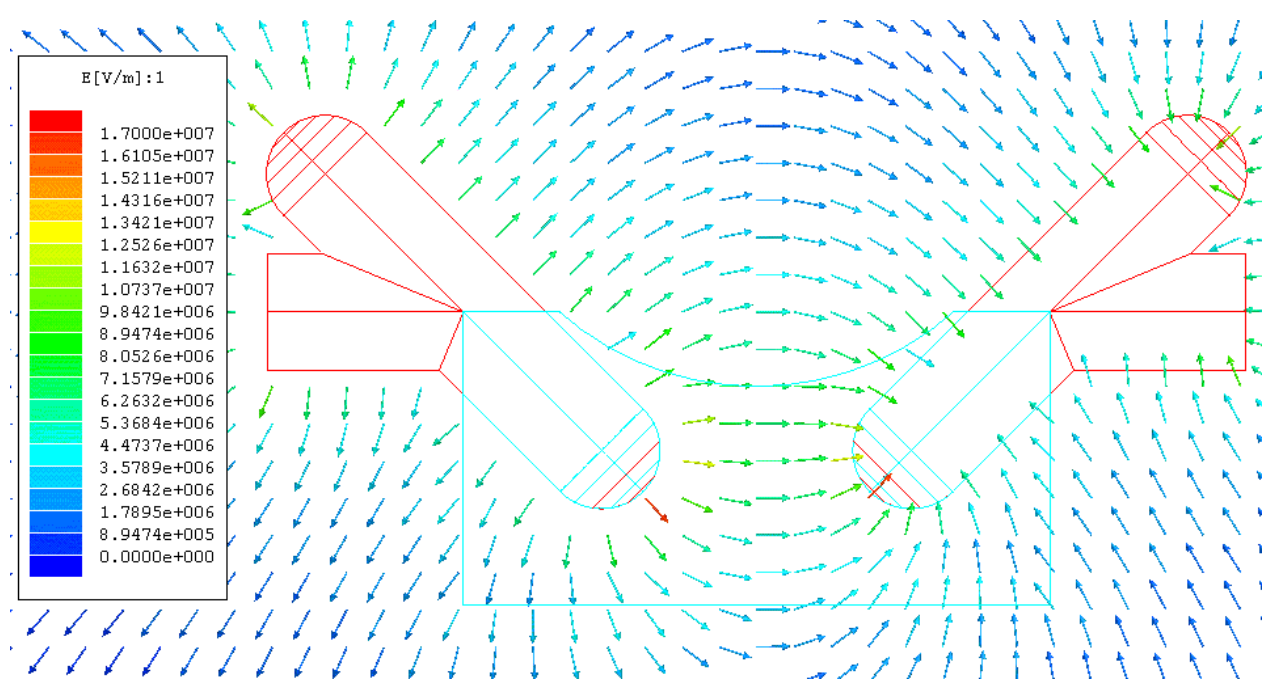

Figure 3.17: Vector plot of the electric field from a side view of the angled electrodes with a grooved surface, gap distance of $8.4 \mathrm{~mm}$, electrodes charged to $\pm 20 \mathrm{kV}$

\subsection{HPM Testing Apparatus}

The HPM surface flashover experiments are conducted utilizing a setup consisting of both WR284 and WR650 waveguide standards [15]. This is done to achieve a radiation pattern similar to free space radiation, while still allowing for the measurement of transmitted power. A representation of the setup is shown in Figure 3.18. 


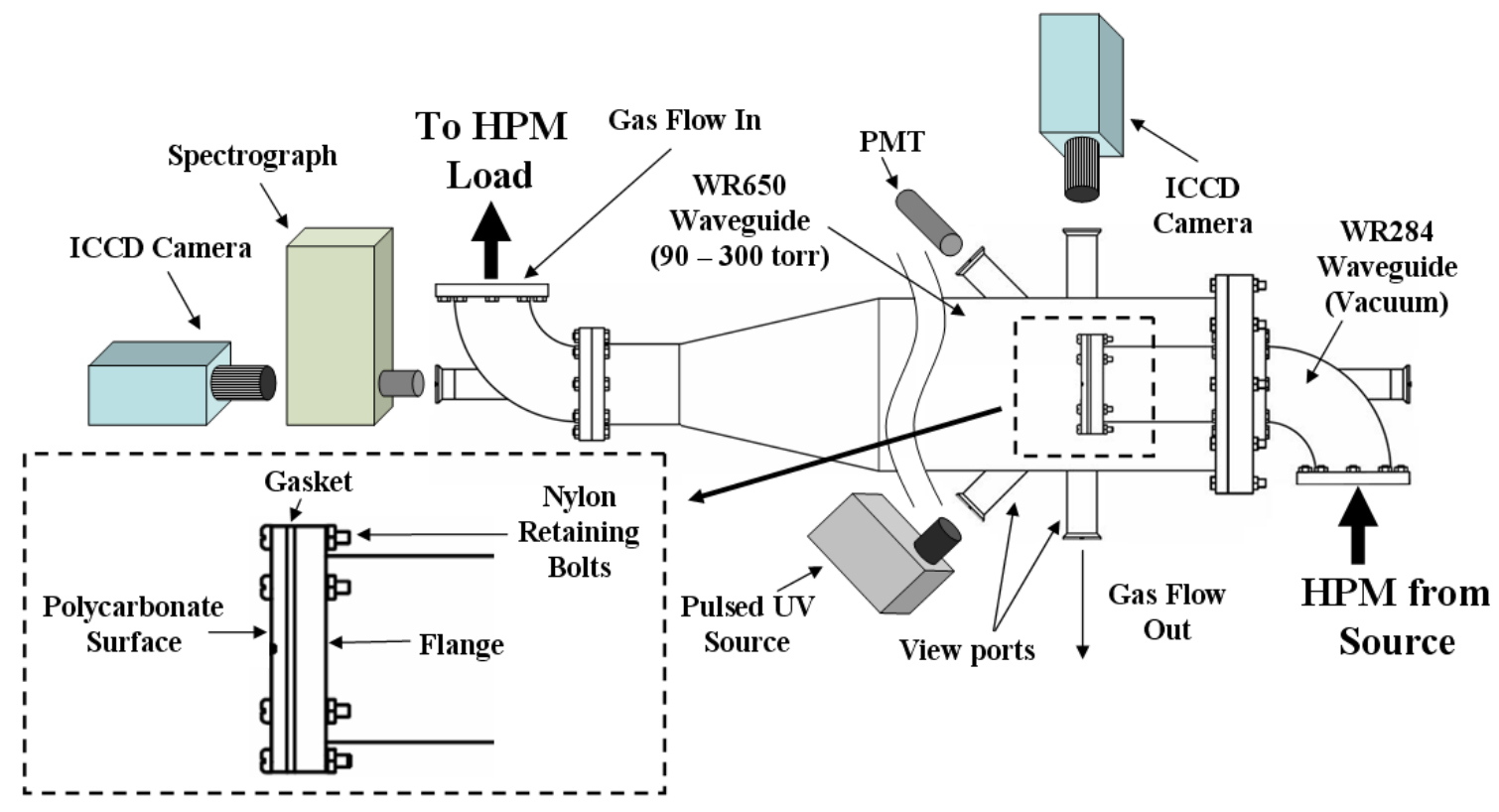

Figure 3.18: Overall system diagram for HPM surface flashover testing. Power measurement diagnostics via a pair of directional couplers in front of the load and after the HPM source are not shown

\subsubsection{Test Chamber}

The HPM travels from the source to the surface under test via a WR284 S-Band waveguide. The HPM source is a US Air Force AW/PFS-6 radar set modified for single pulse operation. A Varian VMS1143B coaxial magnetron, capable of producing 2.85 $\mathrm{GHz}, 2.5-3.5 \mu$ s pulses in excess of $5 \mathrm{MW}$, is the main component of the radar set. Polycarbonate samples used in testing were placed at the radiating end of the WR284 waveguide section. Placed around the polycarbonate sample is a specially designed section of WR650 waveguide allowing for propagation of traveling waves while creating a sealed environment for atmospheric testing. The walls of the WR650 testing chamber are lined with ECCOSORB FDS broad frequency high-loss silicone rubber sheets. These non-conductive silicone sheets have high magnetic and dielectric loss properties and 
prevent breakdown in the tapered WR650-WR284 transition. In addition, the high magnetic and dielectric loss properties of the ECCOSORB FDS material offer a means to simulate an "open-air" radiating structure while maintaining the ability to direct transmitted power for measurement.

All waveguide sections between the polycarbonate sample and the source are either kept at high vacuum or filled with sulfur-hexafluoride to prevent breakdown. Other design features of the testing chamber include four optical viewing ports consisting of two H-plane ports with side viewing access, and two ports with $45^{\circ}$ viewing access. The $45^{\circ}$ viewing ports are primarily utilized for either $\mathrm{CW}$ or pulsed UV illumination of the polycarbonate sample, or for photomultiplier tube, PMT, diagnostics. In addition to the four optical viewing ports, two additional viewing ports are affixed to the test chamber allowing for full view of both the front and the back of the polycarbonate sample.

\subsubsection{Flange/Window Design}

It is desired to determine the impact of the dielectric window in the initiation and growth stages of HPM surface flashover. To that end, it is necessary to isolate the flashover location from other potential dominant processes. A key process to mitigate is the electron injection from the electrodes. The test sample area of the experimental setup was designed to remove all electrodes from areas around the flashover location. Figure 3.19 shows the exit flange geometry utilized to satisfy this design requirement. Eight nylon bolts are used to secure the polycarbonate test sample to the open-ended waveguide. The absence of bolts near the high field regions of the TE10 mode are 
deliberate, as to minimize any effect the presence of the bolt may have on flashover development. Nylon bolts near the sample's vertical axis of symmetry are also removed to allow for an unobstructed side view of flashover events on the surface of the dielectric.

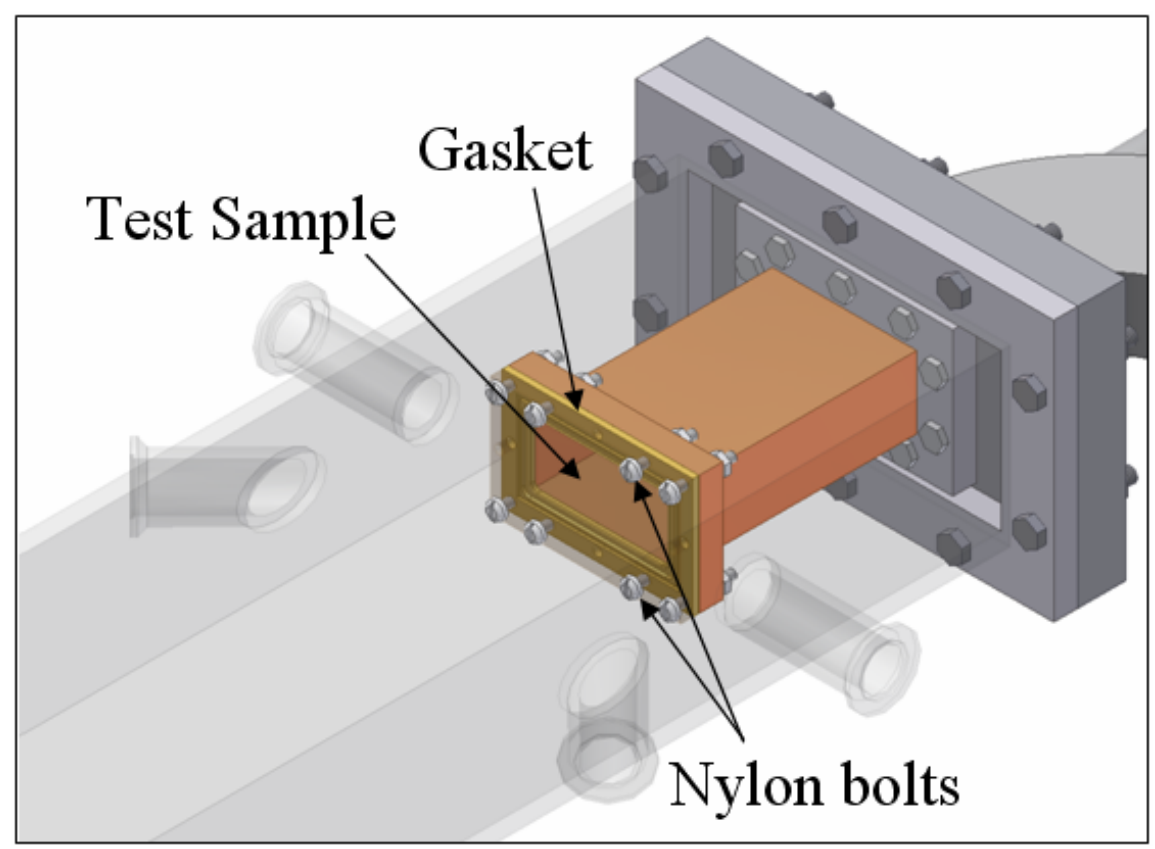

Figure 3.19: Close up view of the exit flange geometry utilized to eliminate metallic conductors from the flashover location [15]

As shown in Figure 3.19, the polycarbonate sample is attached to the WR284 waveguide flange with an intermediary gasket. The purpose of the intermediary gasket is to reduce the field enhancement at the polycarbonate/metal/SF6 triple point inside the waveguide, specifically in the center of the waveguide as this corresponds to the location of the highest E-fields in the TE10 mode. Without this design modification, flashover events tend to initiate from this location. Sufficient reduction in the field enhancement caused by this triple point results in flashover initiating only on the atmospheric side of 
the sample. A variety of gaskets were designed to satisfy this requirement, two of which are shown in Figure 3.20 and Figure 3.21.

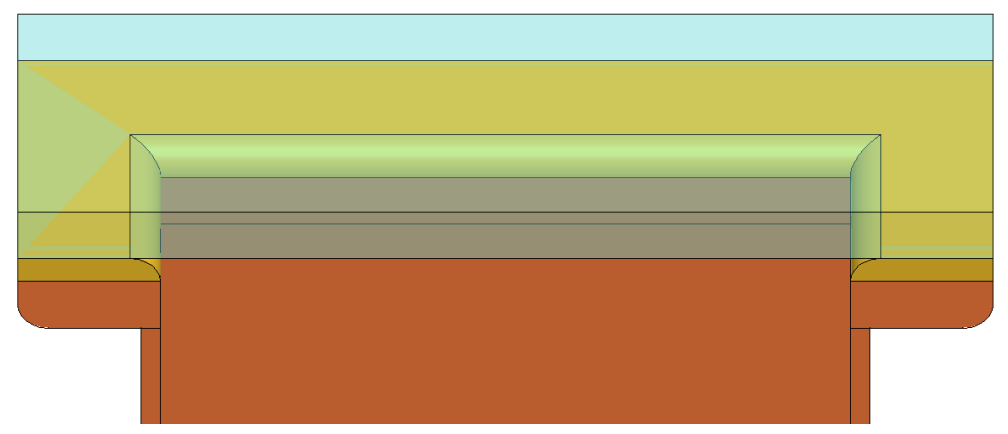

Figure 3.20: Cross section of intermediary gasket design A. The brass gasket is shown between the exit flange and the polycarbonate surface

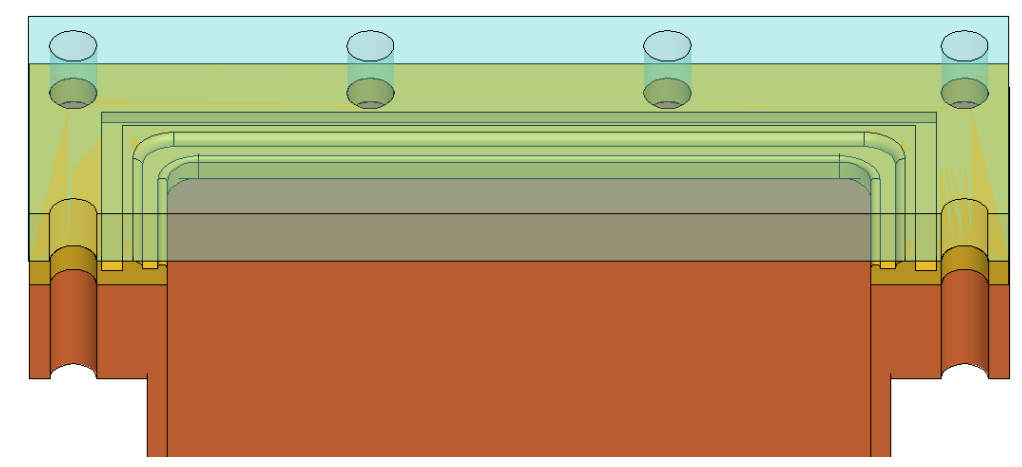

Figure 3.21: Cross section of intermediary gasket design B. The brass gasket is shown between the exit flange and the polycarbonate surface

Simulations of the two gaskets performed in Ansoft HFSS determined that the high field area of gasket B was approximately $50 \%$ lower than the high field area of gasket A. To ensure accuracy, the simulations included the geometry of the entire test section, with all appropriate material properties. Field magnitude and vector profiles of the two intermediary gaskets are shown in Figure 3.22 through Figure 3.25 [15]. The gaskets had minor effects on the system reflection S-parameter (S11), with values of 0.49 and 0.50 
for gaskets A and B, respectively. Incident power for the simulations was 3.25 MW yielding an E-field magnitude at the outside surface of the sample of $14.1 \mathrm{kV} / \mathrm{cm}$ with 10 $\mathrm{kV} / \mathrm{cm}$ normal and $10 \mathrm{kV} / \mathrm{cm}$ tangential field components.

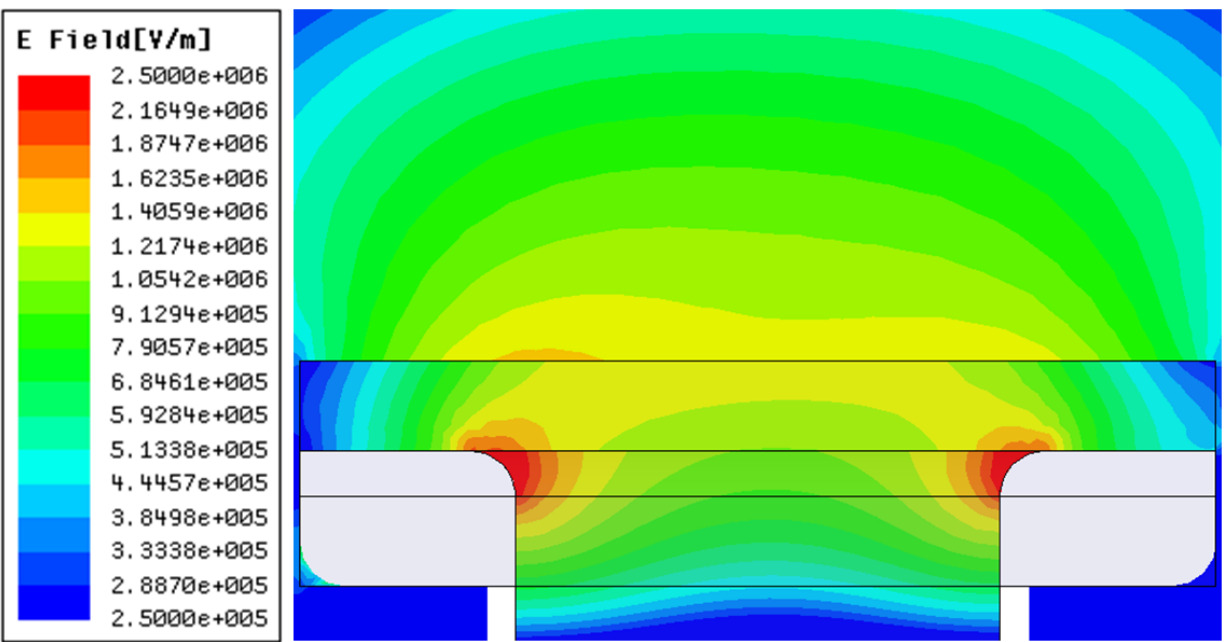

Figure 3.22: Field magnitude profile of intermediary gasket (A) of Figure 3.20 from Ansoft HFSS simulations
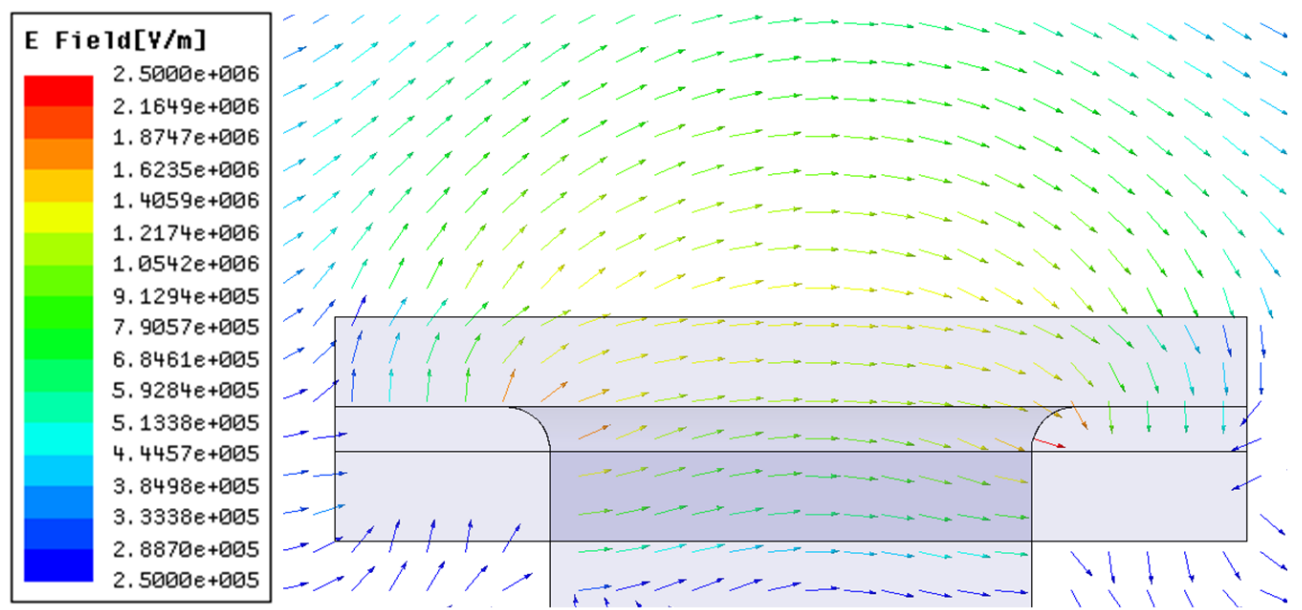

Figure 3.23: Field vector plot of intermediary gasket (A) of Figure 3.20 from Ansoft HFSS simulations 


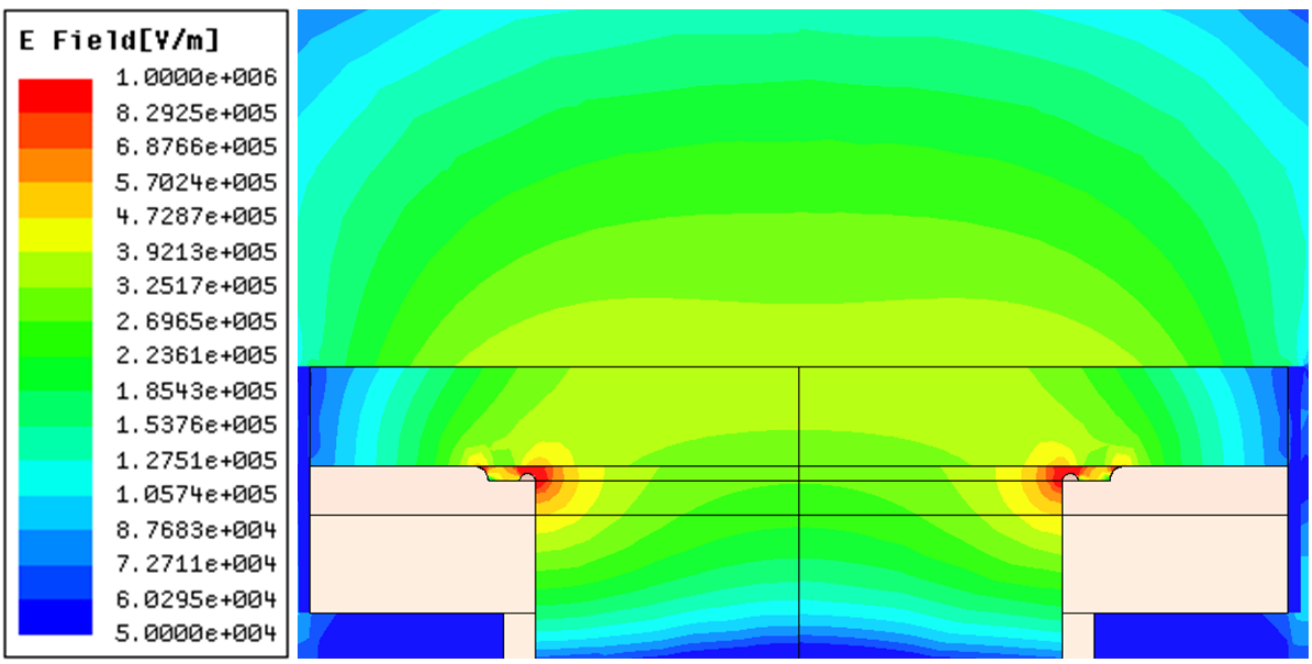

Figure 3.24: Field magnitude profile of intermediary gasket (B) along the A-A cut line of Figure 3.21 from Ansoft HFSS simulations
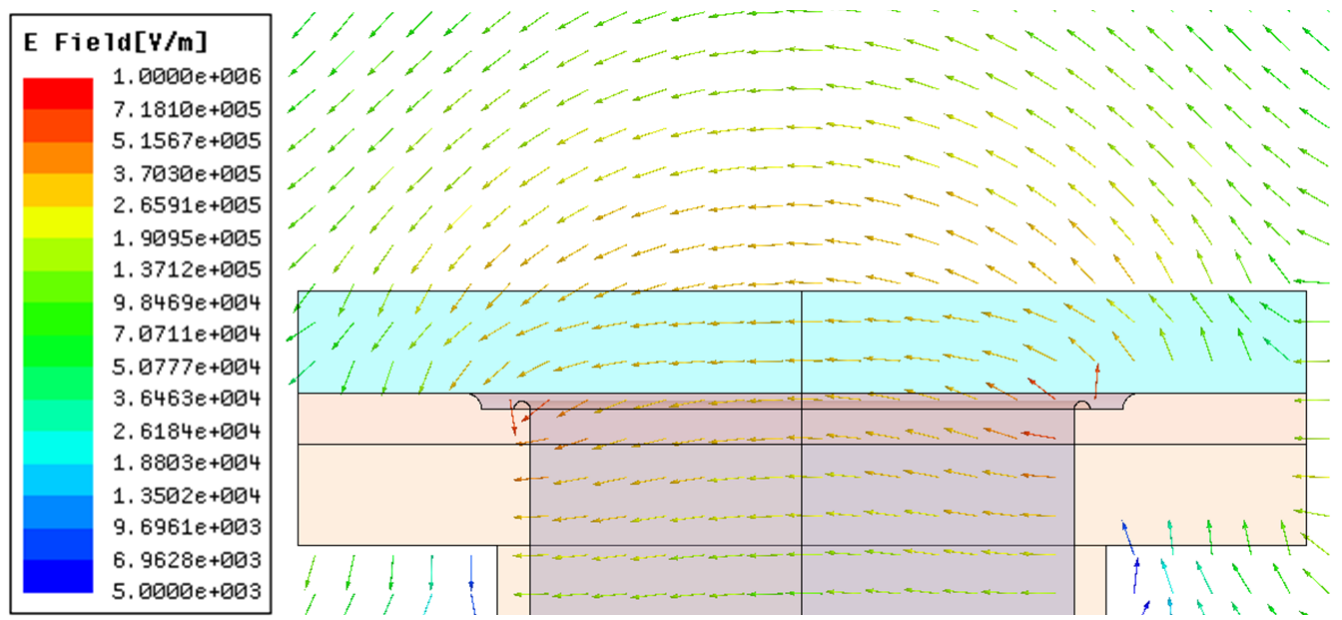

Figure 3.25: Field vector plot of intermediary gasket (B) along the A-A cut line of Figure 3.21 from Ansoft HFSS simulations

The simulations in Ansoft HFSS also provided valuable insight into the peak electric field components related to location on the window surface. Figure 3.26 show the maximum electric field components on the polycarbonate window surface as functions of location. The tangental component of the electric field, $\mathrm{E}_{\text {Tangental, }}$ is comprised of the 
electric field along the surface in the vertical direction, the short axis of the waveguide, due to the fact that there is no horizontal component of the electric field present in the $\mathrm{TE}_{10}$ mode. HFSS simulations confirm the lack of appreciable magnitude in the horizontal direction; therefore this magnitude is not shown in Figure 3.26.

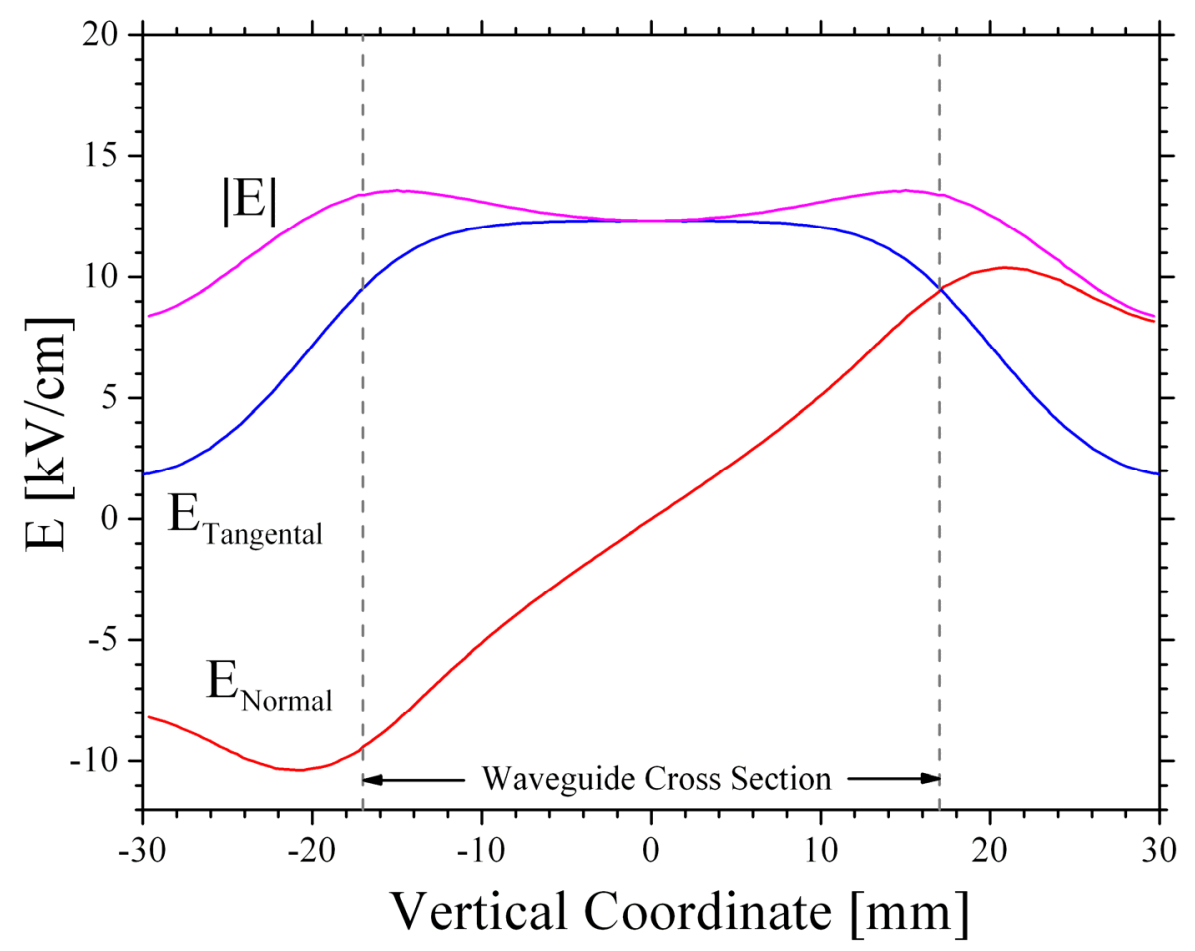

Figure 3.26: E-field components along the polycarbonate window surface as a function of vertical location along the A-A cut line of intermediary gasket B (c.f. Figure 3.21) from Ansoft HFSS simulations, 3.25MW incident power [15]

Testing of the intermediary gaskets with comprehensive luminosity diagnostics on either side of the polycarbonate sample verified that flashover events occur on the atmospheric side prior to any flashover events originating from the polycarbonate/metal/SF6 triple point on the inside of the sample, if any such event should 
occur. Therefore, the design modifications to the intermediary gaskets accomplished the goal of reducing breakdown initiation from the triple point on the $\mathrm{SF}_{6}$ side of the window.

\subsubsection{Environmental Control}

Within the WR650 portion of the test setup several atmospheric variables such as pressure, humidity and gas composition can be controllable. The WR650 section, or testing chamber, can be pumped down to pressures in the torr range while still allowing for continuous gas flow across the polycarbonate sample. Gas composition can be controlled via two inline mass flow controllers. The humidity of the gas flowing into the test chamber is controlled with the setup described previously. Additionally, atmospheric pressure is monitored utilizing MKS Baratron transducers with a 1-1000 torr operating range.

\subsection{Diagnostic Setup}

There are several limiting factors that must be considered in selection and design of the diagnostics sensors. Because unipolar flashover occurs in the nanosecond to subnanosecond time scale the diagnostic sensors must have a temporal resolution in the nanosecond time regime. For the unipolar case the presence of air in the system must be taken into consideration, such as when looking at UV emissions which are absorbed by oxygen. In several cases diagnostic equipment is custom built, as in the case of the light emission gathering apparatus in the unipolar setup. In the HPM case all directional 
couplers and power measurement diodes must be precisely calibrated. All of the major diagnostics are discussed below.

\subsubsection{Current Sensors}

The current sensors used in the unipolar setup are traveling wave current sensors. A detailed discussion of the operation and limitations of these sensors is done by Krompholz [48]. The accuracy of the sensors depends in part on the matched impedance of the entire system to preserve the waveform produced by the closing of the test gap. The sensors are located on the two charging cables near the gap, to minimize the cable transit time from origin of the signal to the sensors. The sensors produce a voltage when a current is applied through them as shown in Figure 3.27. The current sensors have a sensitivity of $0.1 \mathrm{~V} / \mathrm{A}$ and $0.2 \mathrm{~V} / \mathrm{A}$. The sensors are built into a specially designed housing that includes a fitted sleeve that takes the place of the outer conductor on the RG$22052 \Omega$ charging line cable. 


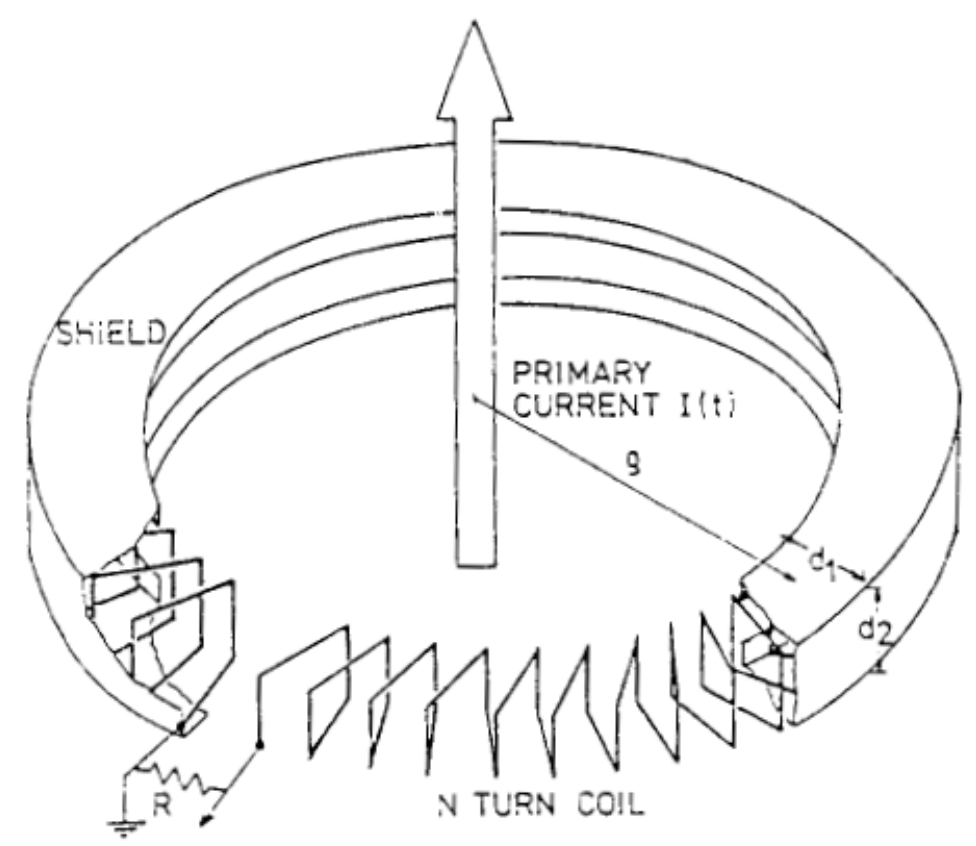

Figure 3.27: Diagram of the traveling wave current sensors [48]

The voltage sensors used in the unipolar setup are capacitive dividers. Capacitive dividers are used for their fast rise time, typically in the hundreds of picoseconds, and their ease of construction on the coaxial geometry of the charging line. The divider is formed by first removing the outer conductor from a small section of the RG-220 charging line cable. A thin strip of copper shim or tape is placed over the insulation of the cable and covered with Kapton tape. A specially designed aluminum "clam shell" is then clamped over the entire assembly, taking the place of the removed outer conductor. This shell has a built in BNC connector with a hole allowing the center pin of the connector to be extended through the Kapton tape to the copper shim. In this way two capacitors are created, the first between the inner conductor and the copper shim with the insulation of the cable as the dielectric. The second is between the copper shim and the outer 
conductor or "clam shell" with the Kapton tape as the dielectric. For some testing a resistor is added in-line with the center pin. As with the current sensors the voltage dividers are located close to the test gap to minimize the cable transit time from origin of the signal to the sensors. A drawing of this arrangement is shown in Figure 3.28.

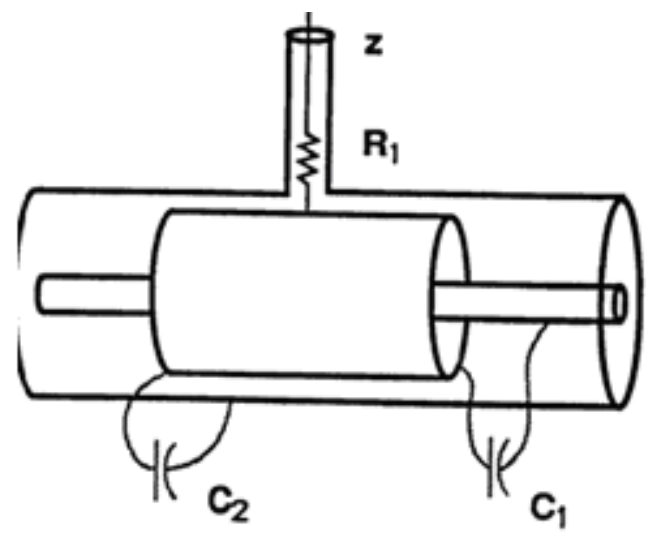

Figure 3.28: Diagram of capacitive voltage divider implemented on a coaxial geometry

\subsubsection{RF Power Measurements}

Power levels in the RF experimental setup are measured via HP 8474B planar-doped barrier diode detectors. Advantages of this diode model include an extremely flat frequency response and very good frequency response stability versus operating temperature. The diodes have a temporal response of $2 \mathrm{~ns}$ and a maximum operating input power of $100 \mathrm{~mW}(20 \mathrm{dBm})$. Diodes are placed on the directional couplers located just before the test window and in front of the high power load. In this way reflected and transmitted power can be measured with respect to the dielectric window. S-parameters for both directional couplers were measured utilizing the Hewlett-Packard HP 8719C network analyzer $(50 \mathrm{MHz}-13.5 \mathrm{GHz})$. All measurement ports required additional 
attenuation in order to prevent exceeding the $20 \mathrm{dBm}$ maximum input power of the diode detectors. In most cases, this additional attenuation was between 40 to $50 \mathrm{~dB}$.

\subsubsection{Optical Diagnostics}

Collecting optical emissions from the arc channel is an important part of understanding the mechanisms involved. For both unipolar and RF setups imaging of the arc is accomplished using special high speed cameras which will be discussed later. In addition to the high speed cameras, several custom built and special ordered components are utilized in the collection of optical emissions as described below.

\subsubsection{Collection Apparatus}

Specialized optical components, specifically a UV transmissive view port and camera lens, are used to focus on the surface from up to a meter away through a small view port as is the case for a frontal view in the HPM setup. Since imaging of the discharge provides just a single picture of the developing discharge, two approaches are implemented to capture spatially resolved light emissions from the discharge, primarily for collection of spectra, for both unipolar and HPM setups. In the HPM setup the slit in the external optics is adjusted to image the entire window height onto the slit and to focus on the center of the arc channel. The imaging capabilities of the spectrograph, which are discussed later, are then used to collect spectra from different points along the arc channel. The regions integrated by the post processing are illustrated in Figure 3.29. 


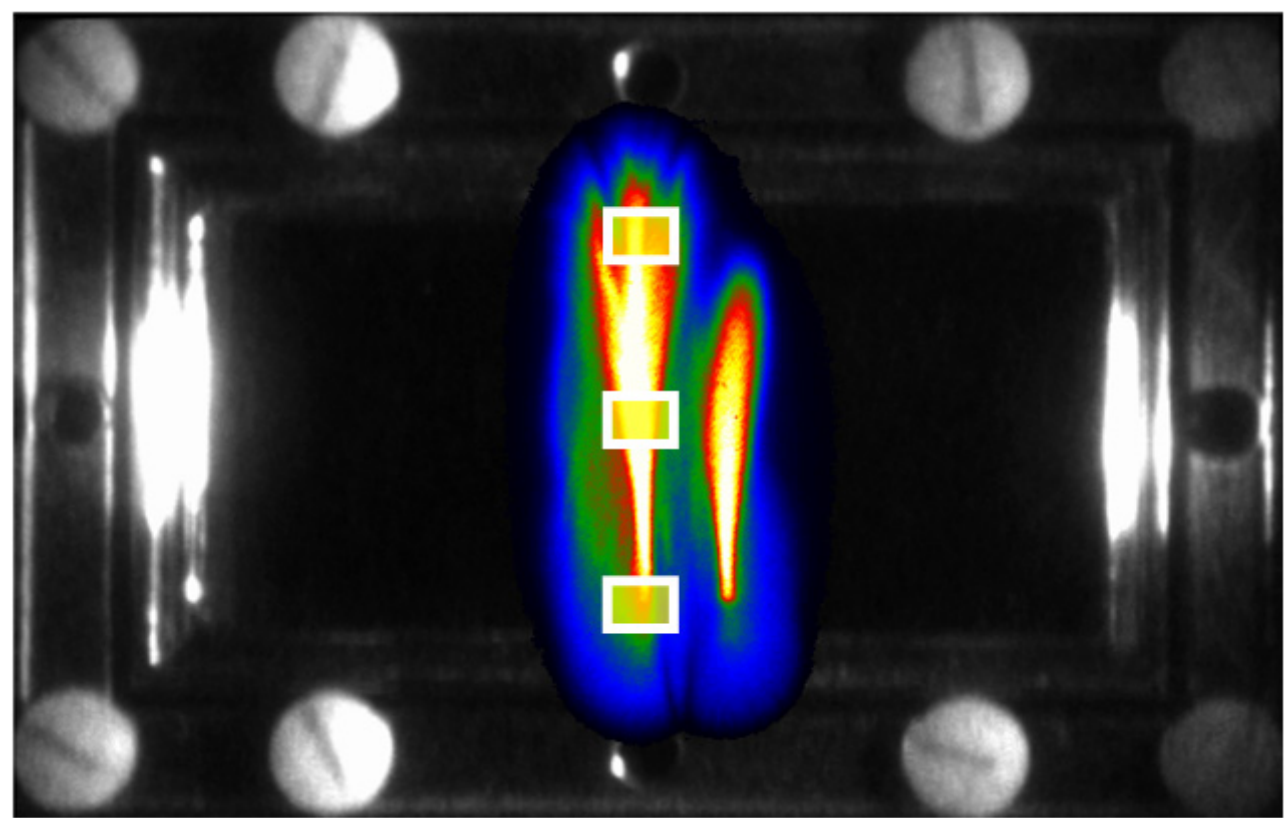

Figure 3.29: Reference image of front of sample with pseudo-color image of a flashover overlaid, spectra is integrated from 3 regions along the arc path as denoted by the white boxes, whose width is exaggerated

In the unipolar experiment a special setup is constructed bearing in mind the necessity of minimizing the distance between the light gathering apparatus and the gap to avoid the absorption of UV by the air. Special considerations are also taken to ensure the UV is not attenuated in the gathering and transportation system for the light, unlike the HPM setup in which the low pressures reduce UV attenuation. The light gathering setup begins with a specially designed lens holder that supports three cylindrical rod lenses shown in Figure 3.30. These rod lenses are made of Suprasil®, which is a high purity synthetic fused silica material, and is transmissive for light above $175 \mathrm{~nm}$. The lenses are $3.0 \mathrm{~mm}$ in diameter and $5.0 \mathrm{~mm}$ long. They are mounted parallel to one another and are $4.95 \mathrm{~mm}$ apart center to center. 


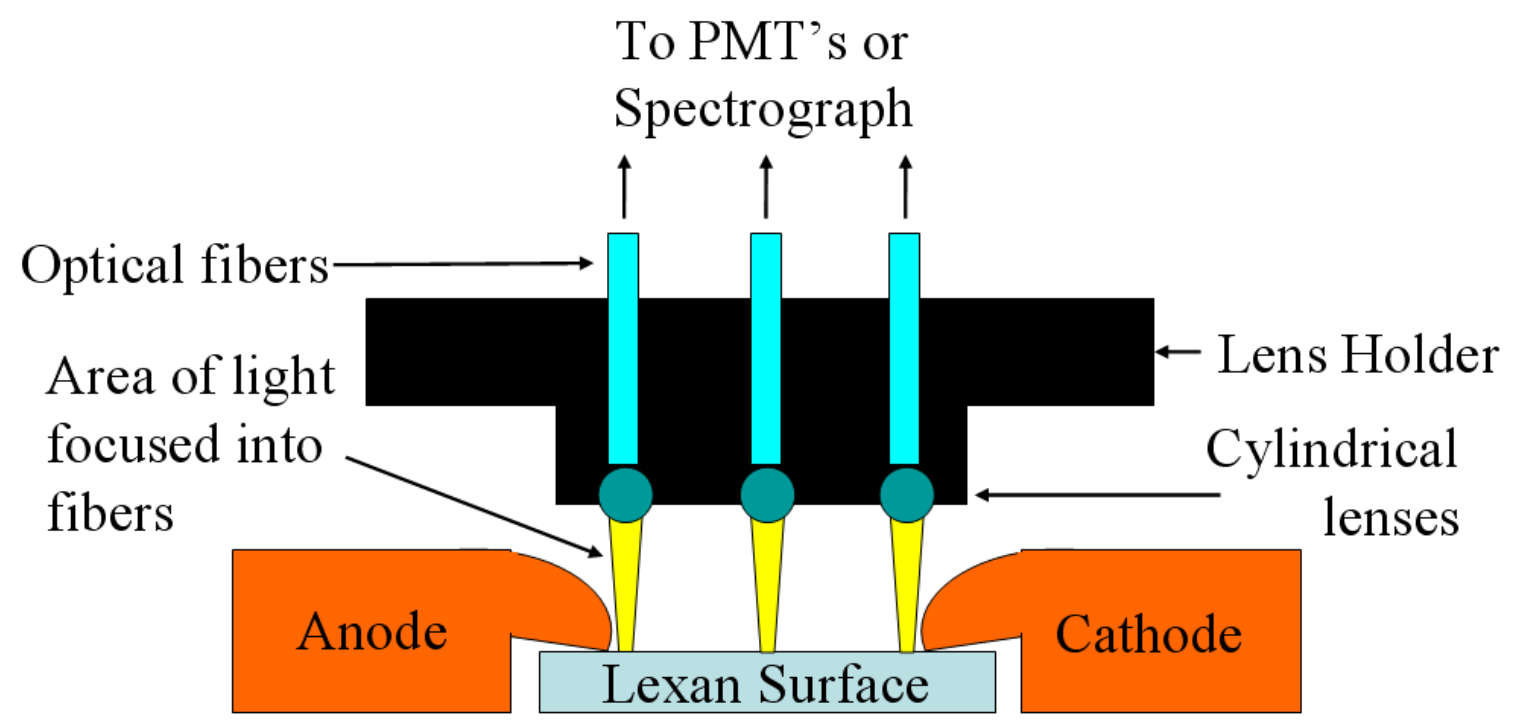

Figure 3.30: Fiber optics setup for spatially resolving light emission in the flashover gap

This places the field of focus of the outside lenses just in front of the two electrodes and the center lens in the center of the gap. Behind each lens is a quartz fiber optic cable into which the lens focuses any collected light. The optical fibers used are Superguide G fibers from Fiberguide industries. The fibers have a $200 \mu \mathrm{m}$ core diameter and a $220 \mu \mathrm{m}$ diameter including the cladding. The fibers contain a low hydroxyl ion content which provides high transmission efficiency from UV through visible and into near IR. The fibers are then fed out of the removable outer conductor through a small hole and connected to a series of feedthroughs located on the side of the test chamber. Connected to the outside of the feedthroughs a special fiber optic assembly feeds the fiber into the spectrograph. The entire lens assembly is held in place above the gap by a polycarbonate mounting bracket attached on either side to the outer conductors of the feedthroughs. Everything fits within the removable outer conductor in order to maintain the systems impedance. 


\subsubsection{Imaging}

An intensified CCD camera, ICCD (Andor Technology) camera is used to image the breakdown. Another ICCD camera is used to record the spectra from a $1 / 4 \mathrm{~m}$ spectrograph. The imaging camera's CCD has 1024x1024 pixels, while the spectrograph's camera has 256x 1024 pixels. The cameras also have a gate controlled micro channel plate shutter with a delay time of $12.0 \mathrm{~ns}$ before opening and $0 \mathrm{~ns}$ before closing. The micro channel is either controlled by the operating system or by a TTL pulse generated by an outside source. The cameras have an adjustable gain from 0.6 to 1315.6 . The imaging camera is mounted either on a rail located to the side of the test chamber or on a fully articulated camera stand. The second camera is mounted to the spectrograph itself. Two computers control the cameras using Andor's MCD software which also collects and stores the images.

\subsubsection{Photo Multiplier Tubes}

The Photo Multiplier Tubes (PMT's) are used to detect the intensity and timing of the light emissions. The PMT's used are Hamamatsu type H6780-04 Photosensor Modules. These PMTs have a fast rise time of $0.78 \mathrm{~ns}$. Each PMT comes from the factory with a different inherent sensitivity; however the gain can be adjusted. Prior to their connection to the test setup the gain voltage is adjusted so the three sensors yield the same output level for equivalent inputs. With the PMTs calibrated to each other, they are enclosed in a small Faraday cage to prevent any outside interference from corrupting the reading. This is necessary because the leads from the PMTs are not shielded, and the PMT's 
inherent sensitivity to noise. A coaxial cable carries the output signals when outside of the Faraday cage. A special header piece is constructed out of black polycarbonate to hold the end of the fiber optic cable in place in front of the PMT and seal out any stray light. The outputs of the PMTs are recorded, along with all other waveforms on the two synchronized digital oscilloscopes. The inherent differences in delay times are accounted for as describe section 3.4.4.

\subsubsection{Spectrograph}

In order to help determine some of the processes contributing to the flashover, a spectrograph is utilized to analyze the emissions from the arc. The spectrograph used is an Oriel MS $2571 / 4$ meter imaging spectrograph. It is equipped with a 4 grating turret and automatic grating switching. The MS 257 is a multi-track or imaging spectrograph. While normal spectrographs are designed for spectral (horizontal) resolution at the expense of vertical resolution, the MS 257 has toroidal mirrors designed to allow multiple vertical points, or fiber optic inputs, to be diffracted at one time, as illustrated in Figure 3.31 and Figure 3.32. Because the F-number of the fiber optic cables, which is 1.67 , is much smaller than the F-number of the spectrograph, which is 3.9, an F-number corrector is attached to the input of the spectrograph. The F-number corrector also holds the output of the three optical fibers in place. The spectrum collected is saved as an intensity map for later analysis. 


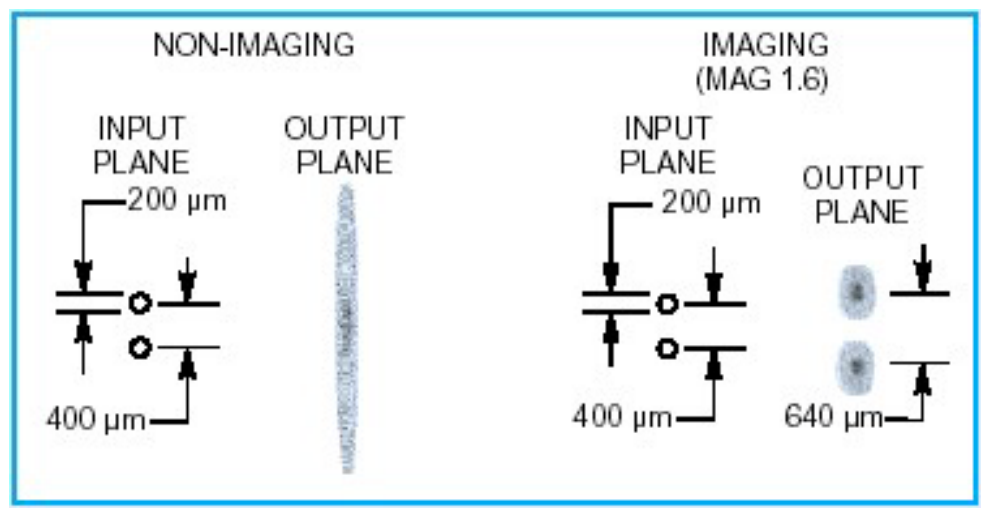

Figure 3.31: Demonstration of difference in imaging and non-imaging spectrographs, imaging spectrographs have specially designed mirrors to maintain vertical resolution

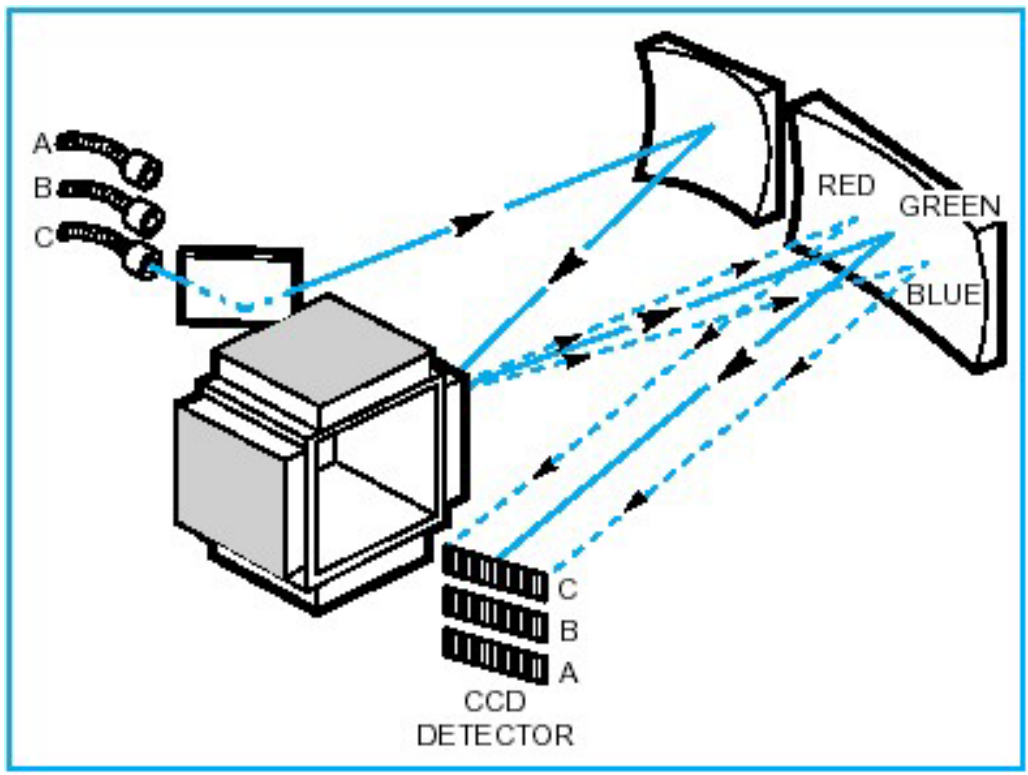

Figure 3.32: Schematic of multi-track spectrograph operation. For the HPM setup direct imaging with a lens is used in place of the fiber optic cables

\subsection{Procedure}

Most of the components used in the experiment remained the same from setup to setup, with only some minor modifications. These components include the voltage, current, and power sensors as well as the PMTs. In addition, at least one of the two 
ICCD cameras was always used. The testing procedure is divided into three parts: surface preparation, electrode preparation, and diagnostic setup. The testing procedure varies slightly depending on which diagnostics are being used.

\subsubsection{Sample Preparation}

The surface preparation consists of cleaning and handling of the samples, which are made from the same material for both unipolar and HPM setups. While the samples are being machined from stock polycarbonate, a protective sticker is in place on the breakdown surface. When the sticker is removed, some residue of the glue still remains to be cleared. Cyclohexane is used to clean the surface of the sample. The surface is thereafter handled with only powder-free latex gloves to eliminate any contamination.

\subsubsection{Electrode / Flange Preparation}

In the unipolar case the electrode preparation involves the cleaning and smoothing of the surfaces. Before each test, the electrodes are examined for any charring or disfiguration. If needed, the electrodes are sanded smooth using 1200 grit sandpaper. The electrodes are then cleaned with methanol and handled thereafter with only powder free latex gloves. Once the electrodes are cleaned, they are inserted into the gap testing chamber. In the case of the hemispherical electrodes, the polycarbonate is then attached via the nylon screws. The threaded holes in the polycarbonate surface are machined exactly the same distance apart on all samples to ensure an equal gap distance. In the case of the angled electrodes, the holes that the electrodes are inserted into are spaced 
equally and drilled to the same depth to ensure equal gap spacing. Once the surface is in place and has been aligned with the camera, the electrodes are tightened onto the mounting pegs with a setscrew to ensure good contact.

In the HPM setup the field shaping flange at the exit of the WR284 waveguide is cleaned and smoothed. At every sample change the flange is checked for excessive pitting caused by arcing on the back side of the sample. This arcing occurs after the flashover event on the front of the sample reflects the power back into the waveguide. If the pitting is pronounced, depending on the power level, the flange will be re-polished and cleaned with methanol prior to being placed in the setup.

\subsubsection{Testing Procedure}

The testing procedure varied only slightly from one experimental setup to another. In all cases the values of attenuators, gas present, humidity, and camera gate time is recorded as well as other setup specific parameters such as charge voltage, gap distance, and electrode type for unipolar testing or pressure, power diode attenuation, and power level for HPM. Also, in all cases, scopes are set for a single shot and armed. After each shot, the wave forms are saved in tabular form on the internal hard drive of the scope, after which the scope is reset. The files are then moved to the network server for storage and future data manipulation. The pressure within the test chamber is checked either for sufficient positive pressure in unipolar or consistent pressure in the HPM case. If humidity experimentation is being conducted, the flow is adjusted until the humidity 
stabilizes at the desired value. Once all conditions are validated the test is repeated until the sample is conditioned, at which time it is replaced.

\subsubsection{Data Manipulation}

The data collected by the scopes, cameras, and spectrograph must first be manipulated to account for certain shortcomings in the test setup. In the case of the waveforms collected by the scopes, the time scales must be shifted to account for different cable lengths leading from the sensors to the scope inputs. In addition, current and voltage signals that are amplified or attenuated must be multiplied by the appropriate scaling factor to reflect the actual values. The power measurement from the diodes on the HPM setup must be scaled to include the attenuation of the coupler and inline power attenuators as well as the response curve of the diodes themselves. Finally, the data collected by the spectrograph must also be brought into a usable form. In the unipolar case each fiber produces a different row of spectra up to 20 pixels high, whereas in the HPM case the rows are chosen to correspond to different locations along the arc channel. In order to identify the peaks, the individual rows of pixels must be integrated and scaled to show the relative peak intensities. This integration is done by a script program written in Andor MCD. The rest of the manipulation and all of the graphs used in this paper are done in PlotIt. 


\section{CHAPTER 4}

\section{MONTE CARLO SIMULATION}

It is desired to simulate the motion of electrons under an external excitation while undergoing retarding collisions with the surrounding gas molecules and surfaces. There are two ways to obtain the electron velocity or energy distribution, either a Boltzmann [49] or Monte Carlo type simulations [50 - 53]. A Monte Carlo simulation has several advantages, including the relative ease of simulating $3 \mathrm{D}$ particle motion, ease of modification to include many electron-molecule and electron-surface interactions, inclusion of oscillating fields, as well as providing insight into the electron cloud formation. In the current implementation, performance is further improved through the use of the null collision method as first laid out by Skullerud $[54,55]$.

In the present case, the simulation includes both surface effects as well as higher pressure regimes ( $>1$ torr) under HPM excitation. HPM excitation is given priority because simulations involving uniform excitation are widely available [3, 56, and 57]. In addition, the electric field profile is custom fit to Ansoft HFSS simulations of the actual HPM test apparatus.

\subsection{Background}

Before beginning to understand the operation of the program it is necessary to understand the basic equation of motion of a charged particle in an external electric field. The velocity at some time $t_{2}$ after $t_{1}$ depends on the electric field, $E$, the frequency of the 
field, $\omega$, and the mass of the electron, $m_{e}$, as in equation (4.1). Starting with the basic equation of force that the electron feels due to the electric field, $F_{e}$, shown in Figure 4.1, and solving for the acceleration, a, results in equation (4.2). Equation (4.3) accounts for the sinusoidal nature for the HPM induced electric field, with $\mathrm{E}_{\mathrm{o}}$ as the max field, $\omega$ as the frequency, $\mathrm{q}_{\mathrm{e}}$ as the charge of the electron, and $\mathrm{m}_{\mathrm{e}}$ as the mass of the electron.

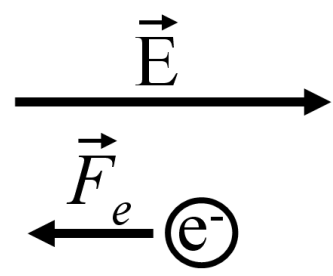

Figure 4.1: Description of force felt by an electron due to an external electric field

$$
\begin{gathered}
\vec{v}\left(t_{2}\right)=\vec{v}\left(t_{1}, t_{2}, \vec{E}, \omega, m_{e}\right) \\
a=\frac{F_{e}}{m_{e}} \\
a=\frac{q_{e} \cdot E_{o} \cdot \cos (\omega \cdot t)}{m_{e}}
\end{gathered}
$$

The acceleration is integrated to yield the instantaneous velocity in equation (4.4). The change in velocity, $\Delta \mathrm{v}$, is determined given a start time, $\mathrm{t}_{1}$, and a time step, $\Delta \mathrm{t}$, as in equation (4.5). If $\Delta \mathrm{t}$ is small compared to the period the equation (4.5) can be simplified to equation (4.6) in the interest of limiting the number of calculations and improving the overall system performance.

$$
v=\int \frac{q_{e} \cdot E_{o} \cdot \cos (\omega \cdot t)}{m_{e}} \cdot \partial t=\frac{q_{e} \cdot E_{o}}{m_{e} \cdot \omega} \cdot \sin (\omega \cdot t)
$$




$$
\begin{gathered}
\Delta v=\frac{q_{e} \cdot E_{o}}{m_{e} \cdot \omega} \cdot\left[\sin \left(\omega \cdot\left(t_{1}+\Delta t\right)\right)-\sin \left(\omega \cdot t_{1}\right)\right] \\
\Delta v=\frac{q_{e} \cdot E_{o}}{m_{e}} \cdot \Delta t \cdot \cos \left(\omega \cdot t_{1}\right)
\end{gathered}
$$

Now that the change in velocity during a given time step can be calculated under free space acceleration the change in position must also be calculated. Equation (4.7) solves for the instantaneous position, $\mathrm{d}$, by integrating equation (4.4), including the initial condition, $\mathrm{v}_{\mathrm{o}}$. The change in position is determined by equation (4.8). The position change equation is them simplified in the same manner as equation (4.5) to yield equation (4.10).

$$
\begin{gathered}
d=\int \frac{q_{e} \cdot E_{o}}{m_{e} \cdot \omega} \cdot \sin (\omega \cdot t) \partial t+\int v_{o} \partial t=\frac{-q_{e} \cdot E_{o}}{m_{e} \cdot \omega^{2}} \cdot \cos (\omega \cdot t)+v_{o} \cdot t \\
\Delta d=\frac{-q_{e} \cdot E_{o}}{m_{e} \cdot \omega^{2}} \cdot \cos \left(\omega \cdot\left(t_{1}+\Delta t\right)\right)+v_{o} \cdot\left(t_{1}+\Delta t\right)-\left[\frac{-q_{e} \cdot E_{o}}{m_{e} \cdot \omega^{2}} \cdot \cos \left(\omega \cdot t_{1}\right)+v_{o} \cdot t_{1}\right] \\
\Delta d=\frac{-q_{e} \cdot E_{o}}{m_{e} \cdot \omega^{2}} \cdot\left[\cos \left(\omega \cdot\left(t_{1}+\Delta t\right)\right)-\cos (\omega \cdot t)\right]+v_{o} \cdot\left(t_{1}+\Delta t-t_{1}\right) \\
\Delta d=\frac{-q_{e} \cdot E_{o}}{m_{e} \cdot \omega^{2}} \cdot \Delta t \cdot \sin \left(\omega \cdot t_{1}\right)+v_{o} \cdot \Delta t
\end{gathered}
$$

Taking into account a 3D geometry and dividing everything into $\mathrm{x}, \mathrm{y}$, and $\mathrm{z}$ components yields equations (4.11) thru (4.16), where $E_{x}, E_{y}$, and $E_{z}$ represent the components of the electric field vector at a given point in space. In this way the velocity and position change of an electron in free space under a given excitation can be calculated. 


$$
\begin{gathered}
v_{x}\left(t_{2}\right)=v_{x}\left(t_{1}\right)+\frac{q_{e} \cdot E_{x}}{m_{e}} \cdot \Delta t \cdot \cos \left(\omega \cdot t_{1}\right) \\
v_{y}\left(t_{2}\right)=v_{y}\left(t_{1}\right)+\frac{q_{e} \cdot E_{y}}{m_{e}} \cdot \Delta t \cdot \cos \left(\omega \cdot t_{1}\right) \\
v_{z}\left(t_{2}\right)=v_{z}\left(t_{1}\right)+\frac{q_{e} \cdot E_{z}}{m_{e}} \cdot \Delta t \cdot \cos \left(\omega \cdot t_{1}\right) \\
d_{x}\left(t_{2}\right)=d_{x}\left(t_{1}\right)+\frac{-q_{e} \cdot E_{x}}{m_{e} \cdot \omega} \cdot \Delta t \cdot \sin \left(\omega \cdot t_{1}\right)+v_{o} \cdot \Delta t \\
d_{y}\left(t_{2}\right)=d_{y}\left(t_{1}\right)+\frac{-q_{e} \cdot E_{y}}{m_{e} \cdot \omega} \cdot \Delta t \cdot \sin \left(\omega \cdot t_{1}\right)+v_{o} \cdot \Delta t \\
d_{z}\left(t_{2}\right)=d_{z}\left(t_{1}\right)+\frac{-q_{e} \cdot E_{z}}{m_{e} \cdot \omega} \cdot \Delta t \cdot \sin \left(\omega \cdot t_{1}\right)+v_{o} \cdot \Delta t
\end{gathered}
$$

Now that the electron's motion in free space can be calculated, the mean free path or, more generally, the time until the next electron-molecule collision occurs, must be determined. For a collision time, $\tau$, a random variable, the equation for the distribution is given by $f(\tau)$ in equation (4.17), where $v_{c}$ is the collision frequency.

$$
f(\tau)=e^{-\int_{0}^{\tau} v_{c} \cdot \partial \tau^{\prime}}
$$

The value, $v_{c}$, is actually the summation of all the collision frequencies for the various types of collisions under consideration, such as elastic, inelastic, attachment, and so on, as denoted in equation (4.18). As can be seen in Figure 4.2, this cumulative collision frequency $v_{c}{ }^{\text {cum }}$ depends on velocity.

$$
v_{c}^{c u m}=\sum_{k=1}^{i} v_{k}
$$




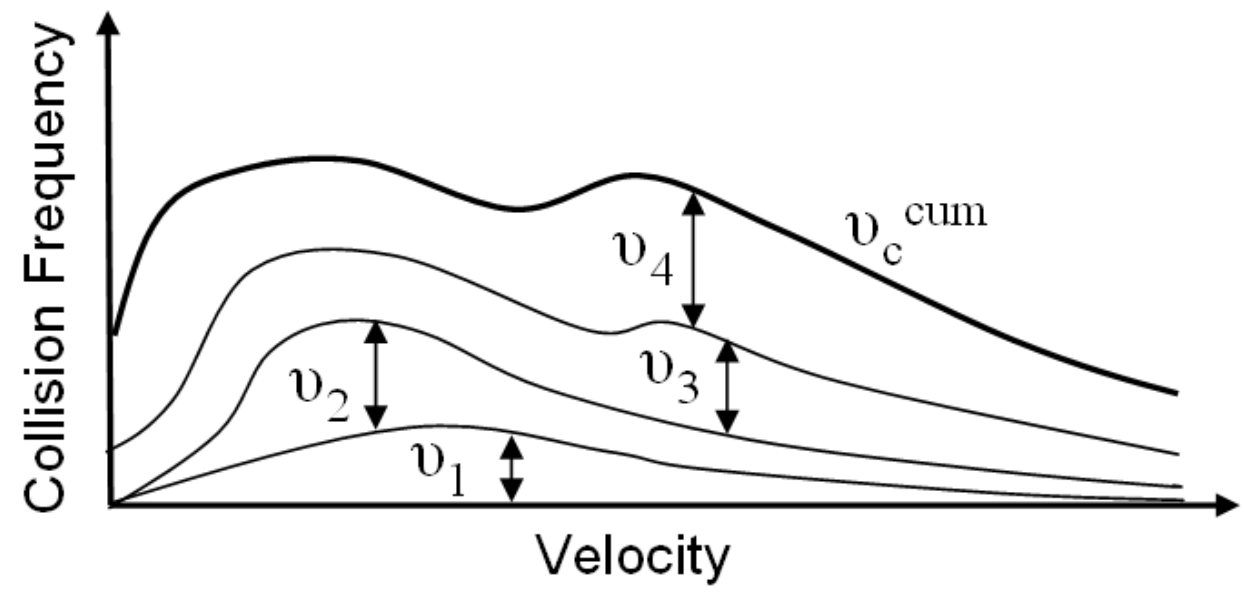

Figure 4.2 The cumulative collision frequency is the addition of the collision frequencies of all considered collisions as stated in equation (4.18)

The value of $\tau$ in equation (4.17) may be chosen randomly by setting $f(\tau)$ equal to a uniformly distributed random number, $r$. However, the collision frequencies' dependence on velocity presents a problem as the velocity at the next collision time depends on the amount of time until the next collision. A solution to this problem, proposed by Skullerud [54], is to introduce a fictitious, or null, collision frequency, $v_{\text {null, }}$ such that when added to all other collision frequencies it results in a constant cumulative collision frequency. This is shown in Figure 4.3. 


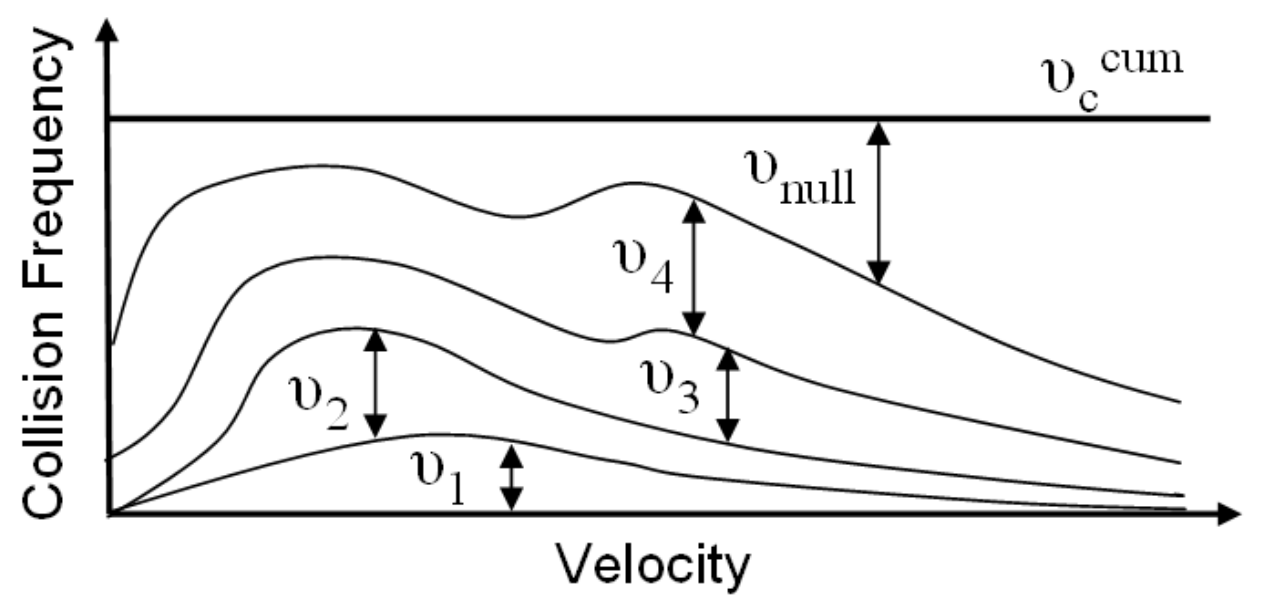

Figure 4.3: Cumulative collision frequency is the addition of the collision frequencies of all considered collisions plus a null collision frequency yielding a constant cumulative collision frequency

As a result of $v_{c}{ }^{\text {cum }}$ being constant, equation (4.17) becomes equation (4.19), and can now be easily solved as shown in equations (4.20) through (4.21), where $\tau$ is the time until the next collision and $r$ is a uniformly distributed random number from 0 to 1 , not including 0. Equation (4.22) yields a Poisson's distribution of collision frequencies as seen in Figure 4.4.

$$
\begin{gathered}
f(\tau)=e^{-\int_{0}^{\tau} v_{c}^{c u m} \cdot \partial \tau^{\prime}}=e^{-\nu_{c}^{c u m} \int_{0}^{\tau} 1 \cdot \partial \tau^{\prime}}=e^{-v_{c}^{c u m} \cdot \tau} \\
f(\tau)=e^{-\nu_{c}^{c u m} \cdot \tau}=r \\
-v_{c}^{c u m} \cdot \tau=\ln (r) \\
\left.\left.\tau=-\frac{1}{v_{c}^{c u m}} \cdot \ln (r) \quad r \in\right] 0,1\right]
\end{gathered}
$$




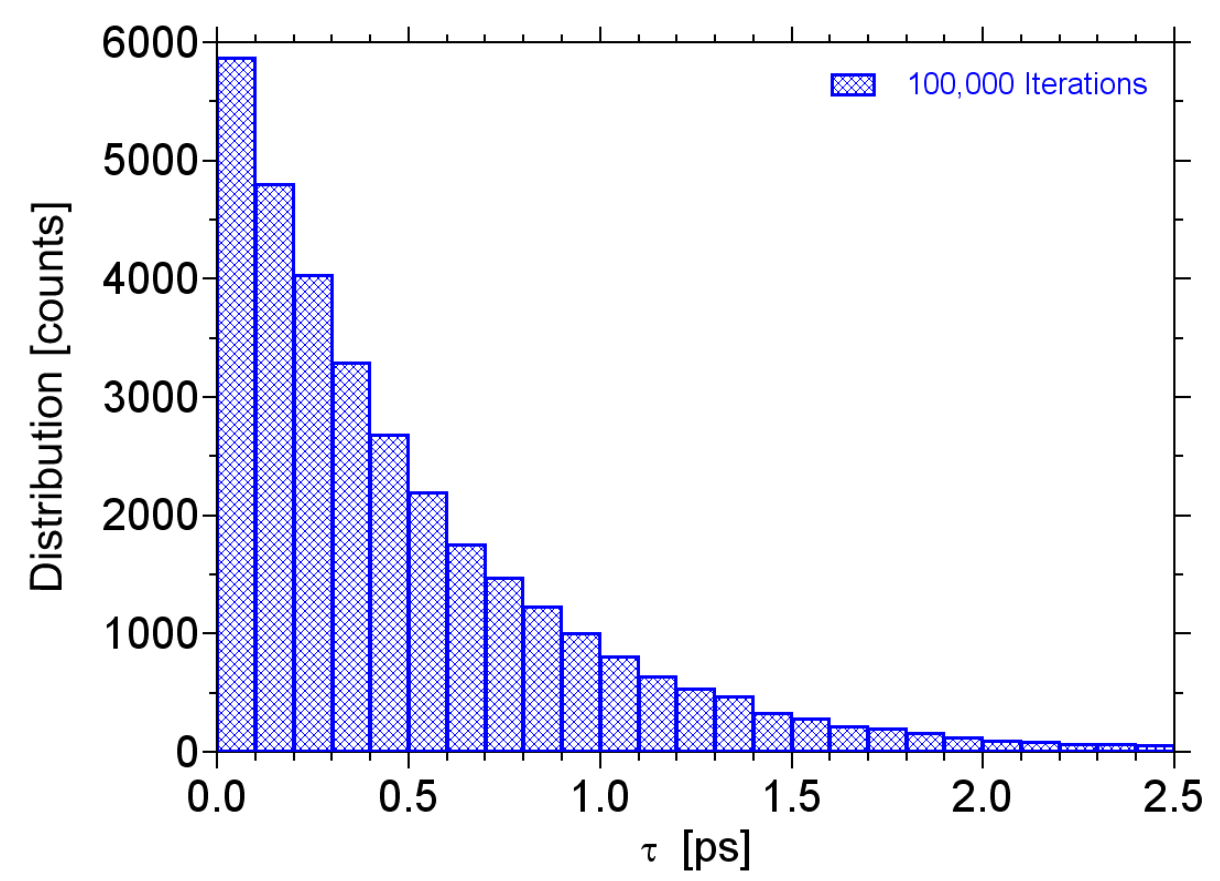

Figure 4.4: Distribution of calculated collision times given a 2 collision/ps cumulative collision frequency and 100,000 iterations

Now that the time until the next collision has been determined, the change in velocity and position of an electron in a given field can be calculated between collisions. Once the simulation reaches the collision time for a particular electron the type of collision must be determined. A uniformly distributed random number is generated and multiplied by the cumulative collision frequency. That value is compared to the values of the various collision frequencies at the electron's velocity. The region the value is contained in is determined to be the type of collision, as illustrated in Figure 4.5. 


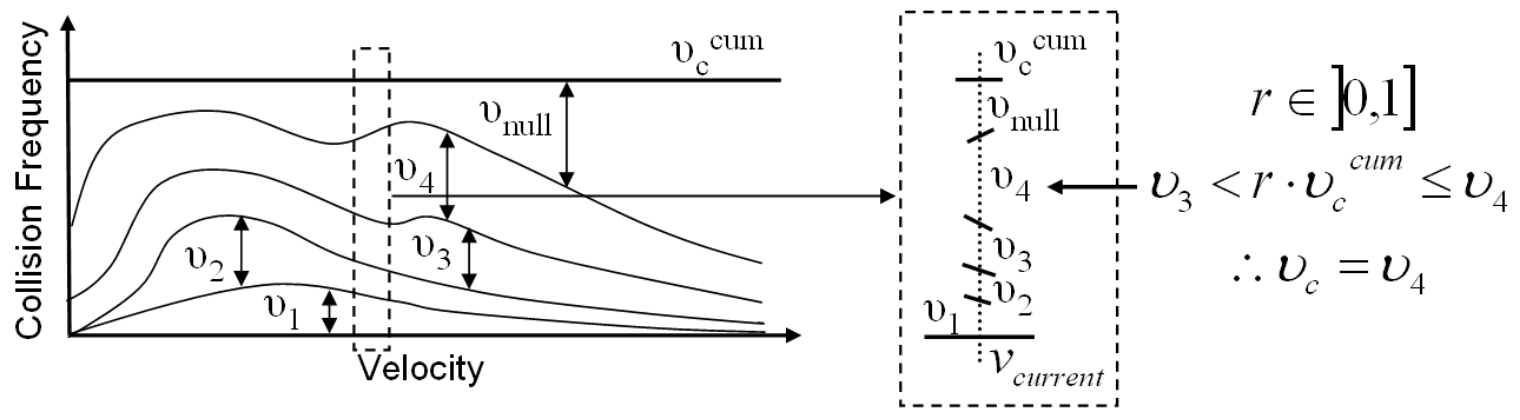

Figure 4.5: Illustration of the determination of collision type using a uniformly distributed random number and the collision frequencies at a given7 velocity

\subsection{Program Operation}

Now that the electron's motion can be tracked between collisions, the next collision time can be found, the type of collision can be determined, and a program can be written to track the motion of multiple electrons under the excitation of a given field in a gas filled volume. Several obstacles still exist in the execution of this program which will be discussed below.

\subsubsection{Program Control}

There are many variables involved in the calculation of the electron's motion and it is desired to be able to change those variables without re-compiling the code. Along this line, the program is designed to read in several commonly altered variables such as pressure, simulation dimensions, time step size, and output window size. These variables are stored in a text file that is read by the program upon initialization. In addition, collision cross sections are read in from comma delimited text files. This allows for the updating and addition of new cross sections as new data is published. These features are 
intended to make the program easier to use, and therefore more useful as a tool in future projects.

The basic parameters, such as maximum electric field, pressure, and initial number of electrons are set via the "Parameter.txt" file, shown in Figure 4.6. While the majority of parameters remain the same from run to run a few of the more commonly adjusted parameters are highlighted and their values will be listed for the various runs.

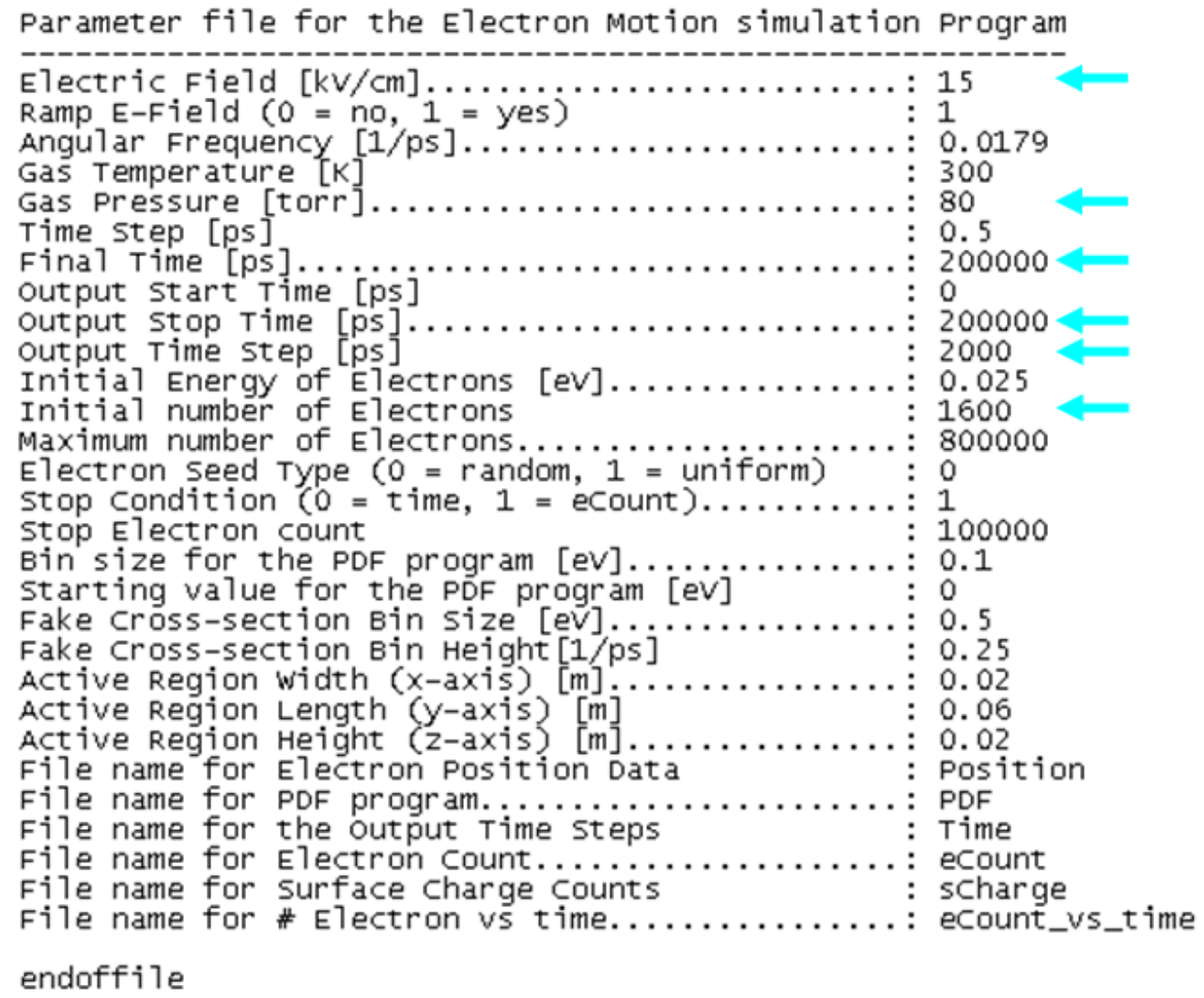

Figure 4.6: Parameter list for giving the simulation control variables. The variables changed most often are denoted by the blue arrows

The other main input the user provides is the cross sections. The actual cross sections are shown in Chapter 2, however, which cross sections are used is controlled by the 
"Cross_Section_List.txt" file. This file also contains information about the associated gases and their relative concentrations, as seen in Figure 4.7. For a pure nitrogen environment the oxygen content is simply set to $0 \%$ and the nitrogen is set to $100 \%$. This brings all oxygen cross sections to zero for all energies.

\begin{tabular}{|c|c|c|c|c|c|c|c|c|}
\hline \multirow{3}{*}{$\begin{array}{l}\text { | Number } \\
\vdots 1 \\
\vdots 2 \\
\text { | Number }\end{array}$} & \multicolumn{2}{|c|}{ IGas Type } & | Percentage [in $x]$ & \multicolumn{5}{|l|}{ |Mass [kg] } \\
\hline & \multicolumn{2}{|c|}{$\begin{array}{l}\text { : vitrogen } \\
\text { :oxygen }\end{array}$} & $: 100$ & \multicolumn{5}{|l|}{$\begin{array}{l}: 4.65 e-26 \\
: 5.300-26\end{array}$} \\
\hline & I туре & IGas & loescription Cho spaces & JlFile name & I of points & I Min Energy & I Energy $70 s 5$ & | B-scatter \\
\hline$: 1$ & $: 1$ & $: 1$ & : Nz_on $7 y$ & :N2_E1a_EEDF.dat & $: 60$ & & $: 0$ & $: 0$ \\
\hline$: \overline{2}$ & $: \overline{2}$ & $: 1$ & : N2 - only & :N2_Ion_NIST, dat & $: 97$ & $: 15.58$ & $: 15.58$ & $: 10$ \\
\hline$: 3$ & $: 4$ & $: 1$ & $: v=-1$ & :N2_vib_vl.dat & $: 50$ & $: 0.29$ & $: 0.29$ & $: 0$ \\
\hline$: 4$ & $: 4$ & $: 1$ & $: v=\overline{2}$ & $: N 2-v i b-v 2$ dat & $: 24$ & $: 0.59$ & $: 0.59$ & $: 0$ \\
\hline$: 5$ & $: 4$ & $: 1$ & $: v=\overline{3}$ & :N2_vib_v3.dat & $: 20$ & $: 0.88$ & $: 0.88$ & $: 0$ \\
\hline$\vdots 6$ & $: 4$ & $: 1$ & $: v=4$ & :N2_wib_w4.dat & $: 18$ & $: 1.17$ & $: 1.17$ & $: 0$ \\
\hline$: 7$ & $: 4$ & $: 1$ & $: v=5$ & :N2 - $w_{1} b_{-} w_{5}$.dat & $: 18$ & $: 1.47$ & $: 1.47$ & $: 0$ \\
\hline$: 8$ & $: 4$ & $: 11$ & $: v=6$ & :N2_vib_v6. dat & $: 16$ & $: 1.76$ & $: 1.76$ & $: 0$ \\
\hline$: 9$ & $: 4$ & $: 1$ & $\begin{array}{l}: v=7 \\
: v=8\end{array}$ & :N2 $-v_{1} b_{1}-v_{8} \cdot d a t$ & $: 18$ & $: 2.06$ & $: 2.06$ & $: 0$ \\
\hline$: 11$ & $\vdots 5$ & $\vdots 1$ & N2_on $7 y$ & N2_Rot_EEDF.dat & $: 160$ & $\begin{array}{l}: 2.35 \\
: 0.01488\end{array}$ & $:_{0}^{2.35}$ & $\vdots_{0}^{0}$ \\
\hline$: 12$ & $\vdots \overrightarrow{6}$ & $: 1$ & :alp & :N2_Ele_alP.dat & $: 29$ & 8.5489 & $: 8.5489$ & 0 \\
\hline$: 13$ & $: 6$ & :1 & : 435 (p) LS) & :N2_E7e_A3S(p) US). dat & $: 31$ & $: 6.1693$ & $: 6.1693$ & $: 0$ \\
\hline$: 14$ & $: 6$ & $: 1$ & aceprime 15 & :N2_Ele_a(2prime)is.dat & $\vdots 25$ & $: 12.253$ & $: 12.253$ & $: 0$ \\
\hline$: 15$ & $: 6$ & $: 1$ & a(prime) 15 & :N2_Ele_a(prime)is.dat & 29 & $: 8.3987$ & $: 8.3987$ & $: 0$ \\
\hline$: 16$ & $: 6$ & $: 1$ & :BSP & :N2_E1 E_BSP.dat & $: 30$ & $: 7.3592$ & $: 7.3592$ & $: 0$ \\
\hline$: 17$ & $: 6$ & $: 1$ & : $\mathrm{B}(\mathrm{pr}$ ime)35 & :N2_E7e_B(prime)35. dat & 29 & 8.1647 & 8.1647 & :0 \\
\hline$: 18$ & $: 6$ & $: 1$ & $\mathrm{CSP}$ & :N2_ETe_CSP. dat & $: 26$ & $: 11.0316$ & $: 11.0316$ & $: 0$ \\
\hline$: 19$ & $: 6$ & $: 1$ & $: E 3 \subseteq(p 7 L 3)$ & :N2 E E EE3S(Plus).dat & $: 26$ & $: 11.8766$ & $: 11.8766$ & $: 0$ \\
\hline$: 20$ & $: 6$ & $: 1$ & :w10 & :N2_E] E-WID.dat & $: 29$ & $: 8.8948$ & $: 8.8948$ & $: 0$ \\
\hline$: 21$ & $: 6$ & $: 1$ & :W30 & : $\mathrm{N2}=1$ E_WBD.dat & $: 30$ & $: 7.3623$ & $: 7.3623$ & $: 0$ \\
\hline$: 22$ & $: 1$ & $: 2$ & :0z_on $7 y$ & $: 02$ E Ela_EEDF.dat & $: 33$ & 0 & $: 0$ & $: 0$ \\
\hline$: 23$ & $: 2$ & $: 2$ & :02_on7y & :02_Ion_NIST.dat & $: 104$ & $: 12.5$ & $: 12.5$ & $: 10$ \\
\hline$: 24$ & $: 3$ & $: 2$ & $: 02+E=02(-)$ & :02_Att_c2si4.dat & $: 651$ & $: 4.41524$ & & $: 0$ \\
\hline$: 25$ & $: 4$ & $: 2$ & $: v=1$ & $: 02$ vib_vl.dat & $: 58$ & $: 0.190$ & $: 0.190$ & $: 0$ \\
\hline$: 26$ & $: 4$ & $: \frac{2}{2}$ & $: v=2$ & $: 02-v i b$ vz. dat & $: 48$ & $: 0.380$ & $: 0.380$ & $: 0$ \\
\hline$: 278$ & $: 4$ & $: \frac{2}{7}$ & $\begin{array}{l}: v=3 \\
: x-4\end{array}$ & $: 02-v i b \_v 3 . d a t$ & $: 45$ & 0.570 & $: 0.570$ & $: 0$ \\
\hline$: 28$ & $: 4,5$ & $: \frac{2}{2}$ & & :02_vib_w4.dat & $: 38$ & $: 0.750$ & $: 0.750$ & $: 0$ \\
\hline$: 29$ & $\begin{array}{l}5 \\
: 6\end{array}$ & $\frac{2}{2}$ & :02-onny & $\begin{array}{l}: 02 \text { Rot_EEDF.dat } \\
: 02 \text { Ele_singletde ta.dat }\end{array}$ & $\begin{array}{l}: 14 \\
: 26\end{array}$ & $\begin{array}{l}0.01 \\
: 0.977\end{array}$ & $: 0.977$ & $: 0$ \\
\hline$: 31$ & $\vdots 6$ & $\vdots 2$ & :stngletsigma & :02_Ele_singletsigma. dat & $: 25$ & $: 1.627$ & $: 1.627$ & 0 \\
\hline$: 32$ & :6 & $\vdots 2$ & $: E_{-} \angle 0 S 5=4.5 \mathrm{eW}$ & :O2_Ele_4_sev.dat & $: 16$ & 4.5 & 4.5 & 0 \\
\hline$: 33$ & $\vdots 6$ & $i_{2}$ & $: E_{-} \operatorname{Las}=6.0 \mathrm{VW}$ & $: 02-E 7$ e_cev.dat & $: 12$ & $: 6$ & $: 6$ & $: 0$ \\
\hline$: 34$ & $: 6$ & $: 2$ & $:$ E_LOSS=8.4 EW & $: 02$ E1e_8_4 ev.dat & $: 9$ & $: 8.4$ & $: 8.4$ & $: 0$ \\
\hline$\vdots 35$ & $\vdots 6$ & $\vdots_{2}$ & $: E_{-} \angle 0 S 5=9.97 \mathrm{VV}$ & $: 02$ E E e_9_97ev.dat & 13 & 0.97 & 0.97 & $: 0$ \\
\hline
\end{tabular}

Figure 4.7: Collision cross section list for identification of cross section to be used. The ratio of gases present in the volume is also controlled here

\subsubsection{Calculations}

The flow of the program is simple, in principle, as seen in Figure 4.8. There are two different step values, the simulation time step and the write time step. The program calculates the electron's motion over the simulation time step, while it only writes to the output at intervals determined by the write time step. This allows for long simulations with simulation time steps much smaller than the electric fields period, while avoiding 
excessive output data. In addition the simulation can be run for long periods of time prior to the beginning of output steps.

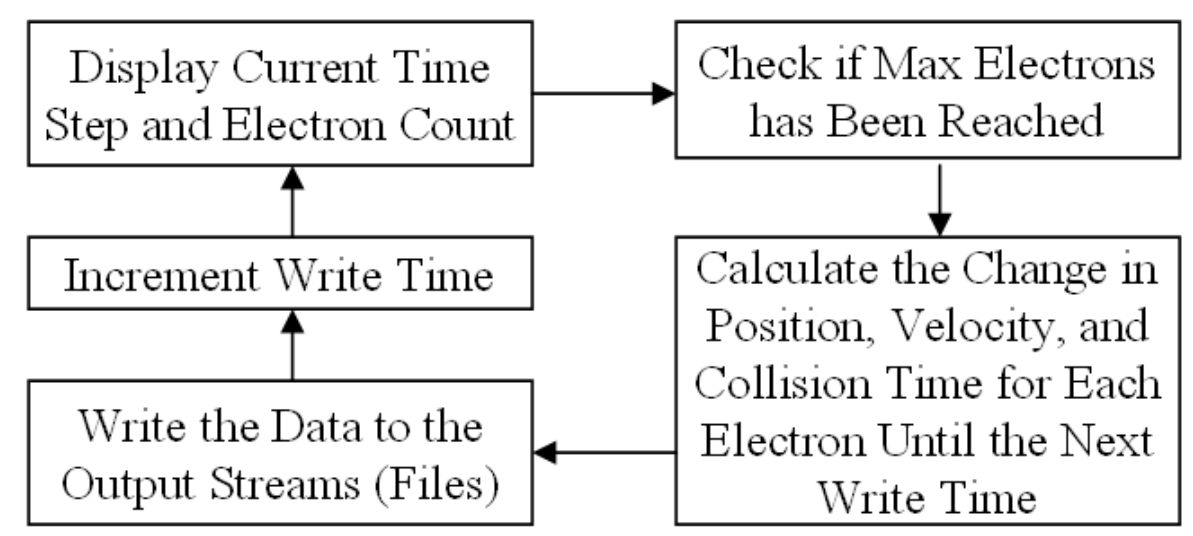

Figure 4.8: Program flowchart illustrating the steps involved in calculating each write time

Within the calculation block in Figure 4.8 is where the change in electron velocity and position is calculated. All changes for a single electron within the simulation time step are calculated prior to calculation of the next electron in the array. If the next collision time for any given electron is greater than the end of the current time step, only the change in velocity and position due to free space acceleration by the electric field is calculated. If the collision time is within the current time step the free space change in velocity and position is calculated up to the collision time, the type of collision is determined, the electron is acted on according to the collision type, and a new collision time is determined. If the new collision time is still within the current time step the previous steps are repeated and, if not, the change in velocity and position due to free space acceleration is calculated to the end of the current time step. The various collision 
types are divided into six basic groups, purely elastic, ionizing collisions, electron attachment, vibrational, rotational, and electronic excitation.

In purely elastic collisions the electron is deflected from its current path by a certain deflection angle determined by the doubly differentiated collision cross-section and a random, uniformly distributed roll angle, as depicted in Figure 4.9. The velocity of the electron is reduced in proportion to the deflection angle, as given in equation (4.23), with $\mathrm{m}_{\mathrm{e}}$ representing the mass of the electron, $\mathrm{m}_{\text {molecule }}$ the mass of the colliding molecule, and $\theta$ the angle of deflection.

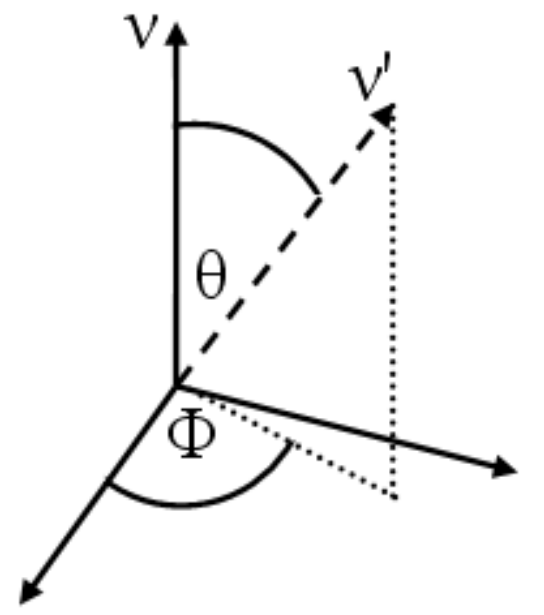

Figure 4.9: Depiction of the definition of deflection, $\theta$, and roll, $\Phi$, with $\mathrm{v}$ being the original direction of the velocity vector and $v^{\prime}$ the resultant vector

$$
\left|v^{\prime}\right|=|v| \cdot\left(1-\frac{m_{e}}{m_{\text {molecule }}} \cdot(1-\cos (\theta))\right)
$$

In an ionizing collision the energies of the scattered incident electron and the generated electron are given by equation (4.24) and (4.25), where $R_{1}$ is a uniformly distributed random number and B is slightly smaller than the ionization energy. An 
electron is only generated if the maximum number of electrons allowed by the simulation has not been reached. The direction of both the scattered and generated electron is randomly generated [51].

$$
\begin{gathered}
E_{\text {scattered }}+E_{\text {created }}=E_{\text {incident }}-E_{\text {ionization }} \\
E_{\text {scattered }}=B \cdot \tan \left(R_{1} \cdot \tan ^{-1}\left(\frac{E_{\text {incident }}-E_{\text {ionization }}}{2 \cdot B}\right)\right)
\end{gathered}
$$

Attachment collisions are easily handled, as no change in direction or velocity is necessary. Originally the program would remove the attached electron and shift the rest of the array up. In the interest of computational time and because the position of an electron within the array does not affect its operation, the electron on the end of the array is moved into the attached electron's position while all other electrons are left as they are. After the electron is moved from the end of the array, the time is reset to the beginning of the current step and the electron is operated on as usual.

In vibrational and rotational collisions the electron loses a set amount of energy depending on the type of collision. The electron is then deflected by an angle determined by the doubly differentiated collision cross-section. The roll angle, as described above, is determined by a uniformly distributed random number.

Finally, in the case of an electronic excitation collision the electron is operated on as it would be in a vibrational or rotational collision. In an electronic excitation it is possible to produce a photon when the molecule returns to a lower energy state. This photon can then excite other molecules, photoionization; however, in the pressure regime of interest this effect is minimal and can be ignored for simplicity. These photons can also impact 
the surface and release electrons. In early stages of the program this effect is also ignored, but will be included in later versions of the code.

\subsubsection{Output}

After a given output time step is reached several parameters are recorded such as the number of electrons, the current time step, the electrons position, the electron energy distribution, and the number of electron impacts on the surface. As the program is executed on a cluster of 16 machines, and the slowest part of the system is the networking between nodes, communication between nodes and between the nodes and the main hard drive should be kept to a minimum. In an effort to keep this communication small, each node stores its results locally; once all calculations are complete each node copies its results files to the main hard drive. When all the pieces are on one hard drive, the main node assembles the files into single output files and deletes the pieces. 


\section{CHAPTER 5}

\section{RESULTS}

Given the large volume of data collected and the similarities of certain waveforms for a given excitation, such as the incident pulse on the system, a summary of all waveforms collected is given instead of showing all nearly identical waveforms in a given series. For delay time testing the voltage and current sensor pair is used to measure both the voltage applied to the gap and current through the gap at breakdown, as seen in Figure 5.1 for a $26 \mathrm{kV}$ incident pulse. The droop in the voltage waveform is an artifact of the fast capacitive voltage divider. For imaging tests, the current sensor is used to trigger the camera and the camera's gate pulse, temporally correlated to the breakdown, is recorded instead of the current waveform.

For HPM excitation several power measurements are recorded along with luminosity from the PMT. Figure 5.2 shows a typical set of waveforms from a HPM surface flashover at 5MW. The early stages of the surface flashover are denoted by a "spike" in the 100 times amplified luminosity signal followed by a rise in the un-amplified luminosity signal as seen in bottom half of Figure 5.2. A drop is detected in the incident transmitted and reflected power waveforms shortly after the initial luminosity rise. The reflected power signal drops upon initiation of the flashover before rising several $100 \mathrm{~ns}$ later. This drop in reflected power with a corresponding drop in transmitted power suggests that power is being consumed by the developing discharge plasma. The rise in reflected power indicates a plasma density sufficient to reflect the incident microwave 
energy. The flashover delay time is measured from the initial rise in the incident power to the drop in reflected power.

It is important to note that the waveforms shown are only intended to provide a general representation of breakdown waveforms. The actual waveforms vary in magnitude and rise/fall time for different test conditions.

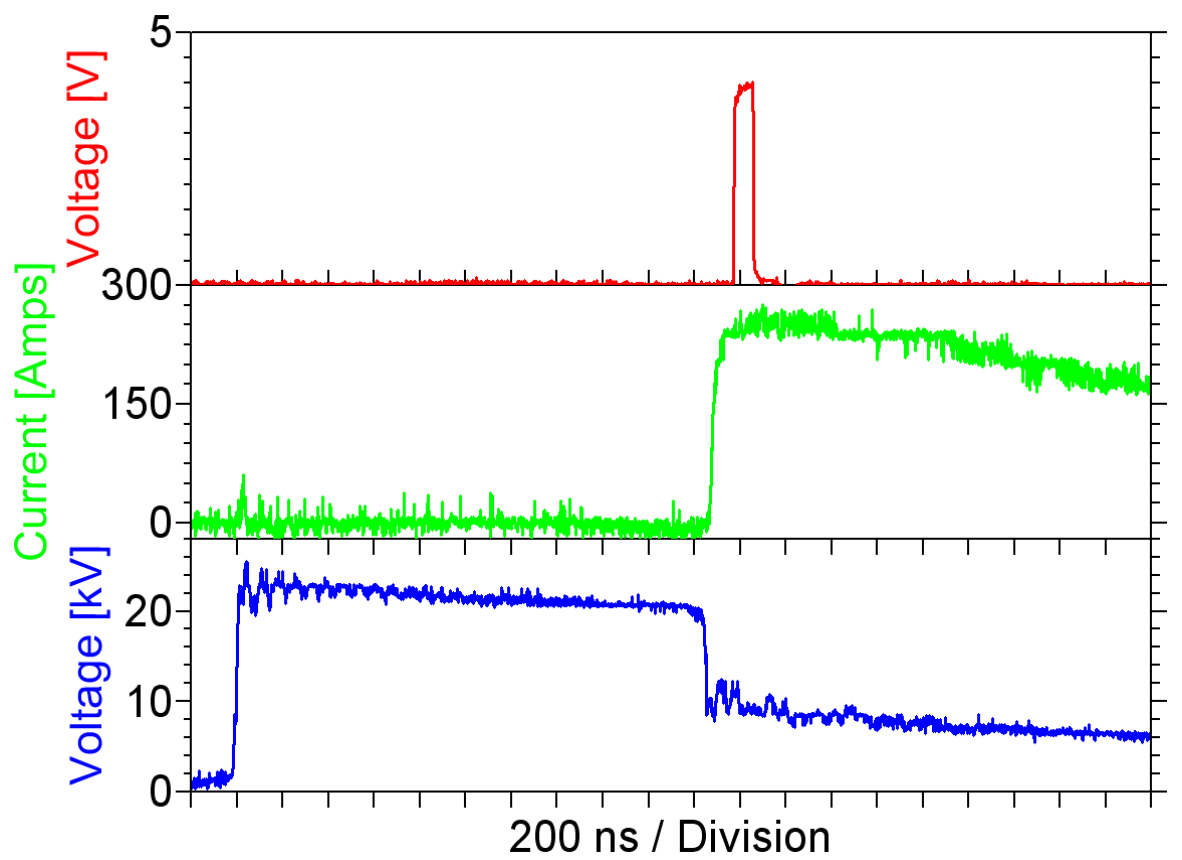

Figure 5.1: Current and voltage waveforms of a typical pulse with flashover. The top plot shows the camera trigger pulse. The middle plot shows the current rise at flashover. The bottom plot depicts the incident pulse and the voltage drop at flashover 


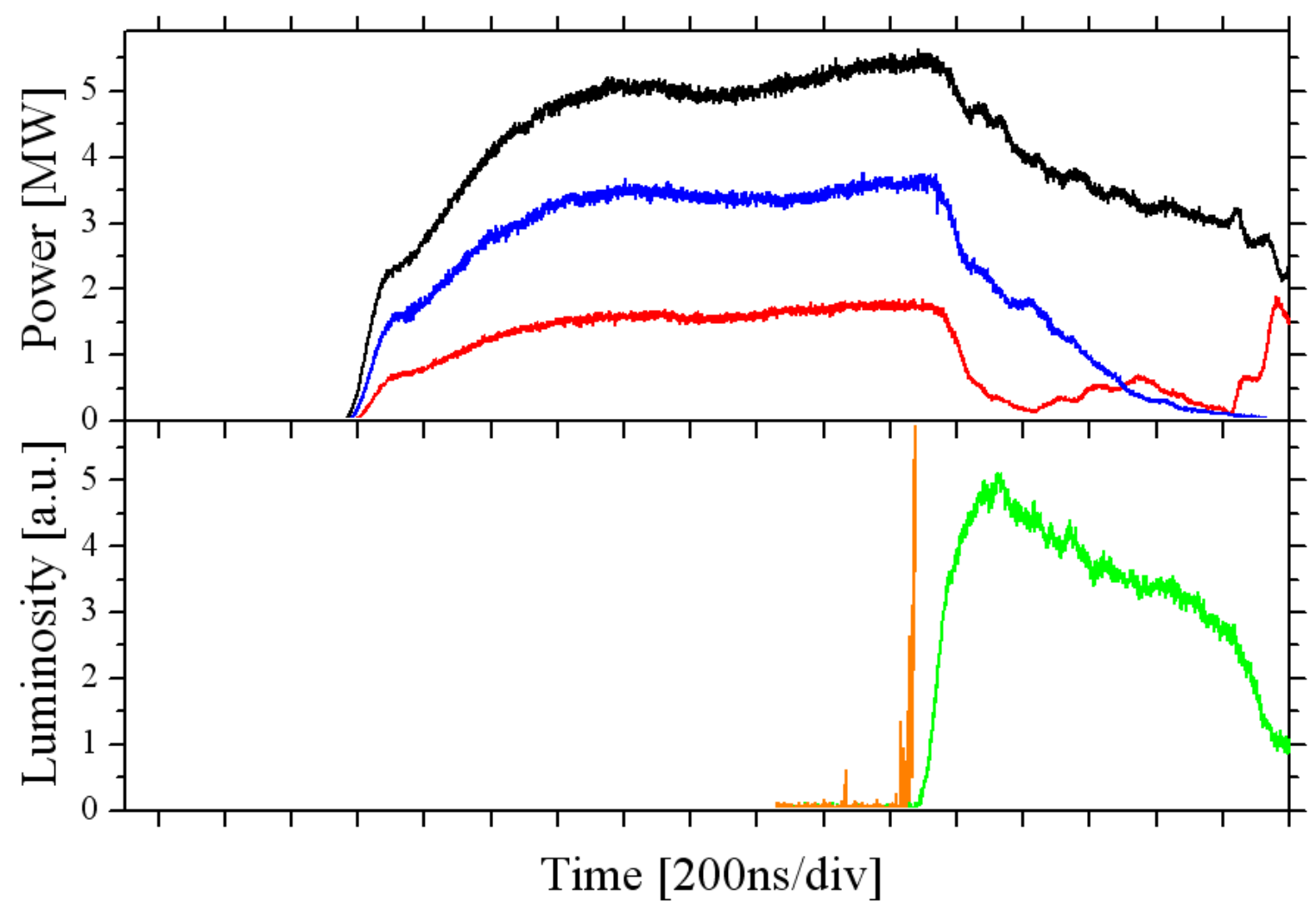

Figure 5.2: Power and luminosity waveforms for a typical HPM surface flashover. The top graph shows the incident power (black), the transmitted power (blue) and the reflected power (red). The bottom graph shows the luminosity in green and the amplified luminosity in orange [15]

\subsection{Arc Channel Formation}

Initial imaging of the arc development under DC unipolar excitation with the hemispherical electrodes showed the arc formation occurring only along the surface, as expected, given that the electric field lines lead into the surface [47]. In order to determine whether the arc is following the surface or the field lines, the angled electrodes are utilized to create a field path away from the surface. Figure 5.3 and Figure 5.4 show the arc formation near the surface in an air environment while forming along the field 
lines in a pure nitrogen environment. This held true for both DC and pulsed unipolar excitation of the gap [14].

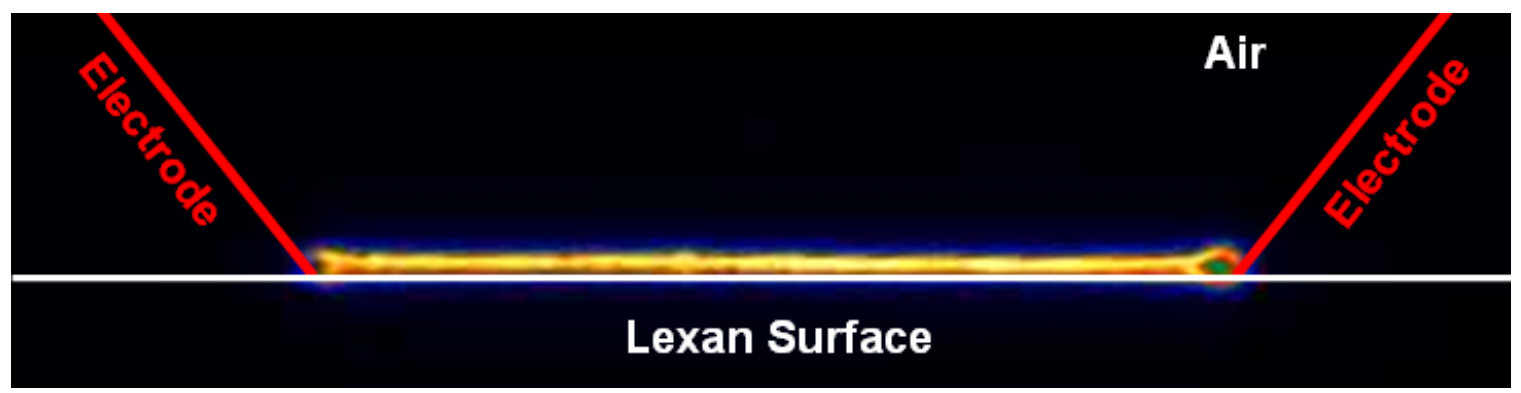

Figure 5.3: Side-on pseudo color intensity plot of main breakdown in air with a polycarbonate surface and angled electrode, $8.1 \mathrm{~mm}$ gap. Pulse voltage, $\mathrm{V}_{\mathrm{p}}=25 \mathrm{kV}$

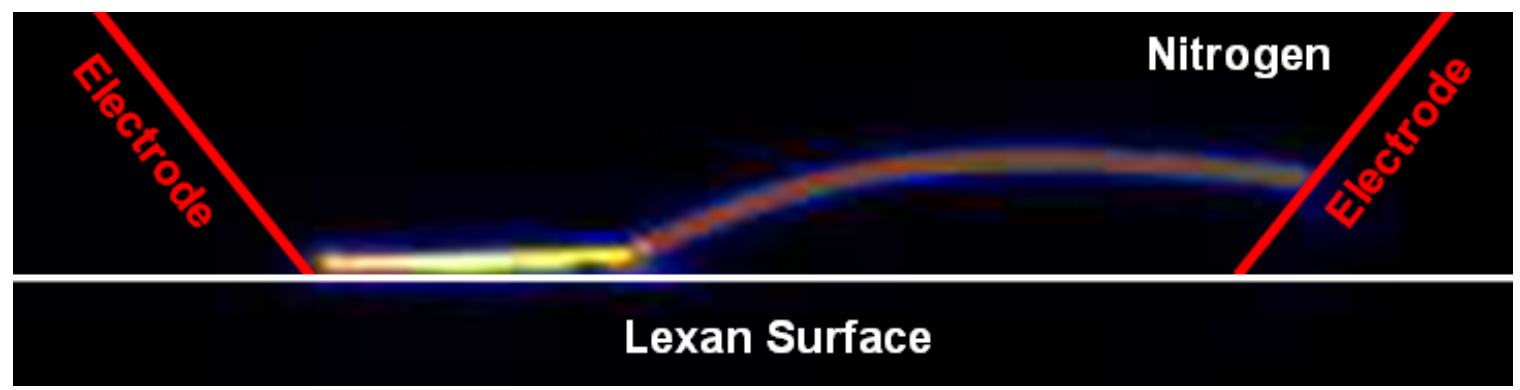

Figure 5.4: Side-on pseudo color intensity plot of main breakdown in nitrogen with a polycarbonate surface and angled electrode, $8.1 \mathrm{~mm}$ gap. Pulse voltage, $\mathrm{V}_{\mathrm{p}}=25 \mathrm{kV}$

In the case of a flat dielectric surface the path length along the electric field lines is longer than the path along the surface. While the arcs in nitrogen show a strong tendency to form away from the surface despite the longer path length, an arc in air might follow the field lines if the path lengths were similar. In order to increase the surface distance a groove is cut into the sample surface. This sets the two path lengths to roughly the same value. As seen in Figure 5.5 and Figure 5.6, the arc in air continued to follow the surface 
while the arc in nitrogen continued to follow the field lines. Again this holds true for both DC and pulsed unipolar excitation.

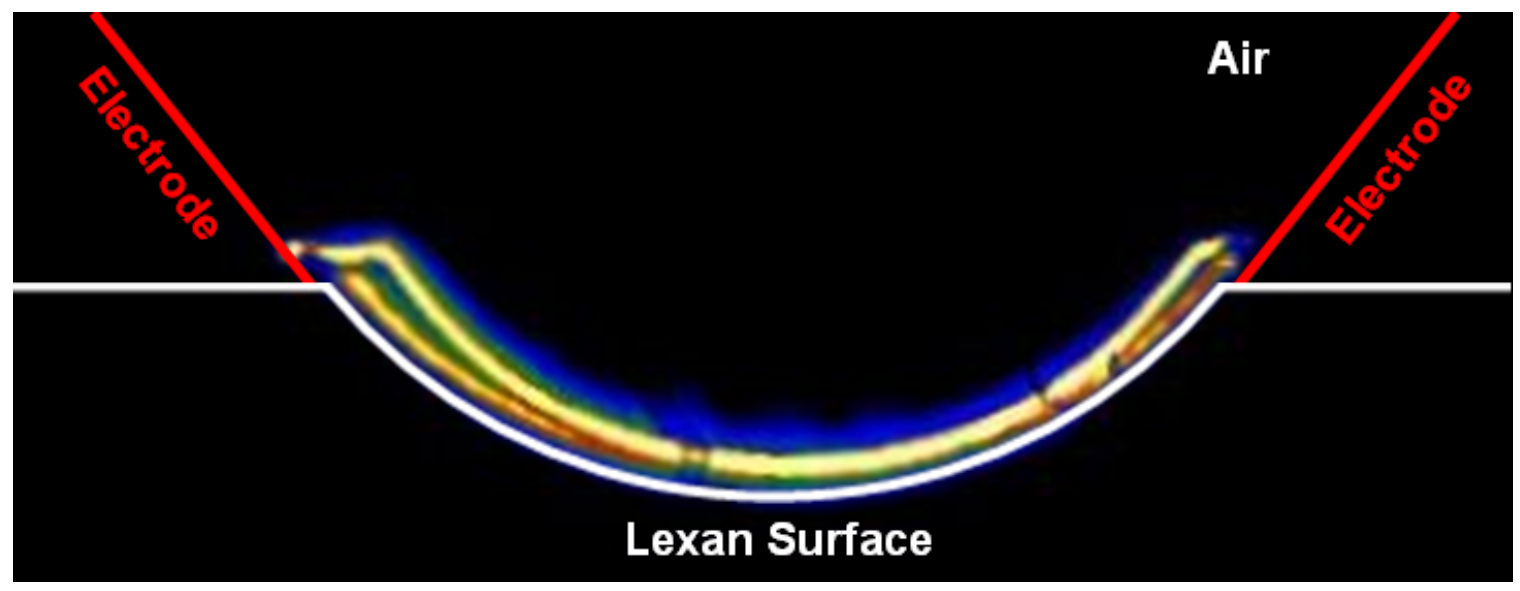

Figure 5.5: Side-on pseudo color intensity plot of main breakdown in air with a polycarbonate surface and angled electrode, $8.1 \mathrm{~mm}$ gap. Pulse voltage, $\mathrm{V}_{\mathrm{p}}=25 \mathrm{kV}$

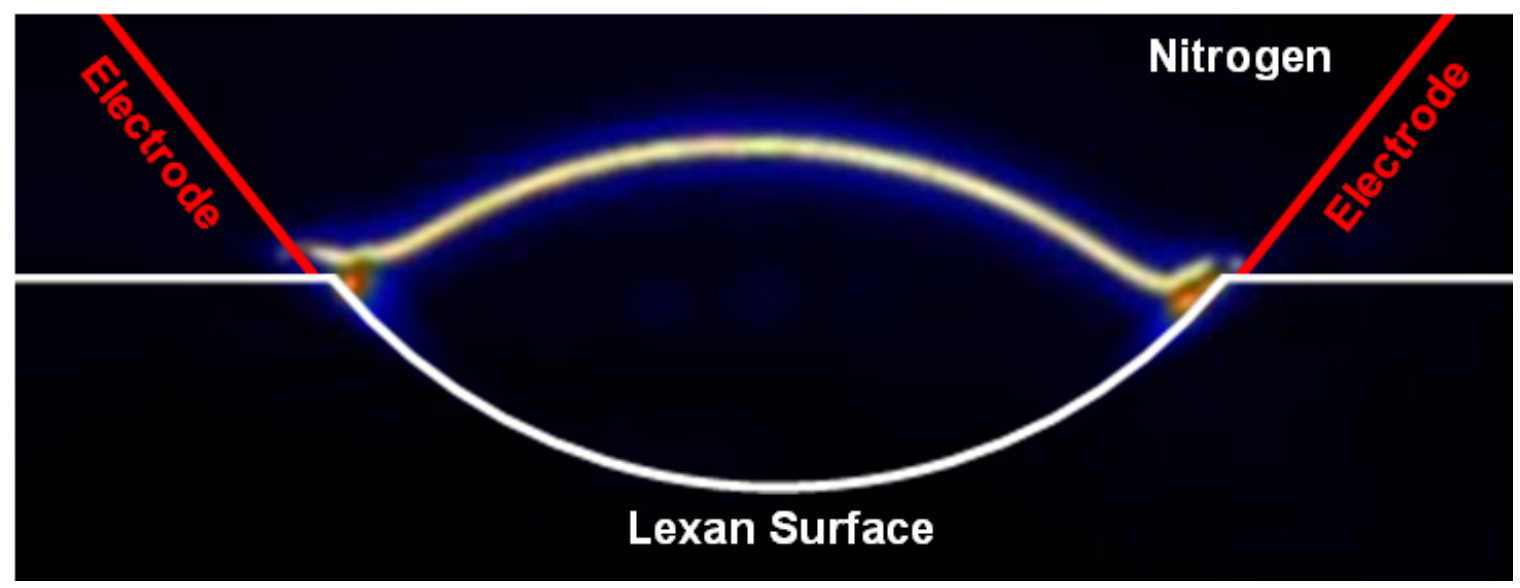

Figure 5.6: Side-on pseudo color intensity plot of main breakdown in nitrogen with a polycarbonate surface and angled electrode, $8.1 \mathrm{~mm}$ gap. Pulse voltage, $\mathrm{V}_{\mathrm{p}}=25 \mathrm{kV}$

Given the similarities in the electric field profile between the angled electrodes and the HPM exit flange, a similar result is expected in the arc channel formation. Imaging of the surface flashover under HPM excitation does show a tendency for the arc to lift off 
the surface in a nitrogen environment while forming close to the surface in an air environment, as seen in Figure 5.7 and Figure 5.8.

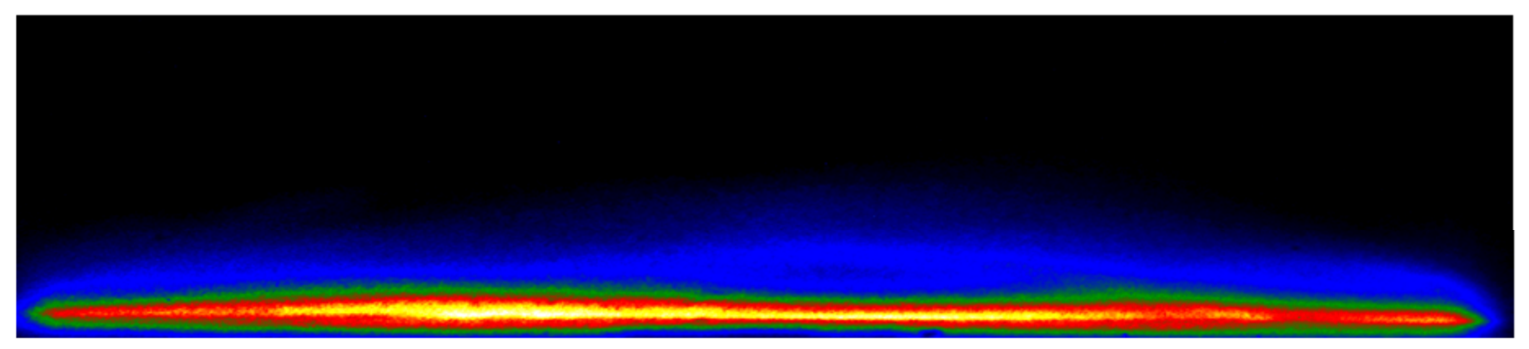

Figure 5.7: Side-on pseudo color intensity plot of main breakdown in air with a polycarbonate surface under HPM excitation

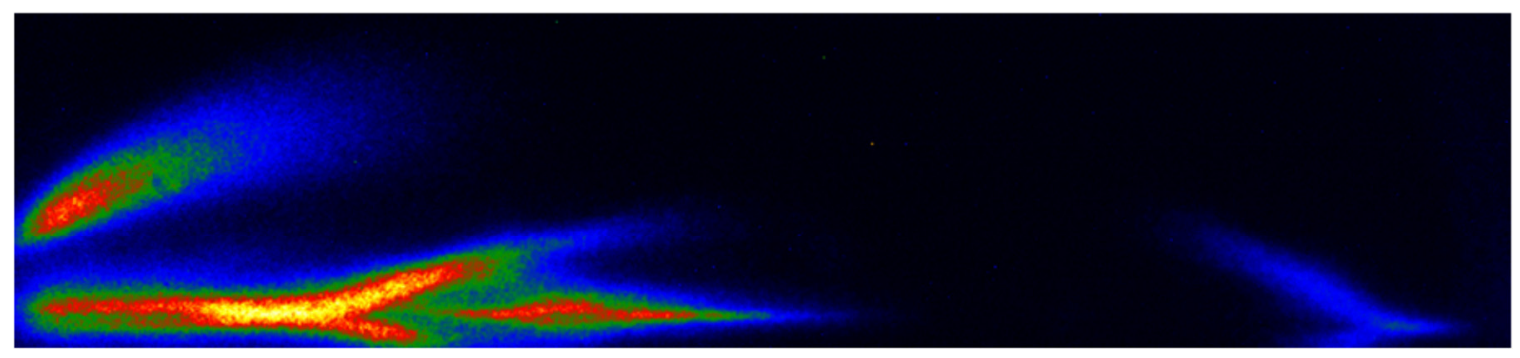

Figure 5.8: Side-on pseudo color intensity plot of main breakdown in nitrogen with a polycarbonate surface under HPM excitation

The liftoff phenomenon is attributed to the lack of oxygen in the pure nitrogen environment. It is postulated that the arc formation is affected in two ways. First, an arc in oxygen produces more UV radiation than an arc in nitrogen. The UV illuminates the surface and produces photoelectrons from the surface. As a result, there is a higher concentration of electrons close to the surface available for streamer formation. Secondly, the scavenging of free electrons by the electronegative oxygen will further suppress the development of the arc in the volume. Any electrons generated at the surface will remain within 2 or 3 free path lengths $(\sim 1 \mu \mathrm{m})$ from the surface on the timescale of interest. 
Imaging of the arc formation under HPM excitation from the frontal view shows the tendency of the flashover event to form in the high field region at the center of the waveguide, as seen in Figure 5.9 below. These images, referenced to the rise in the reflected power, represent several shots with varying camera delays. Optical emission is detected prior to the rise in reflected power, as denoted by the negative time delay of the first arc image. It is important to note that all imaged flashover events started near the center of the waveguide.
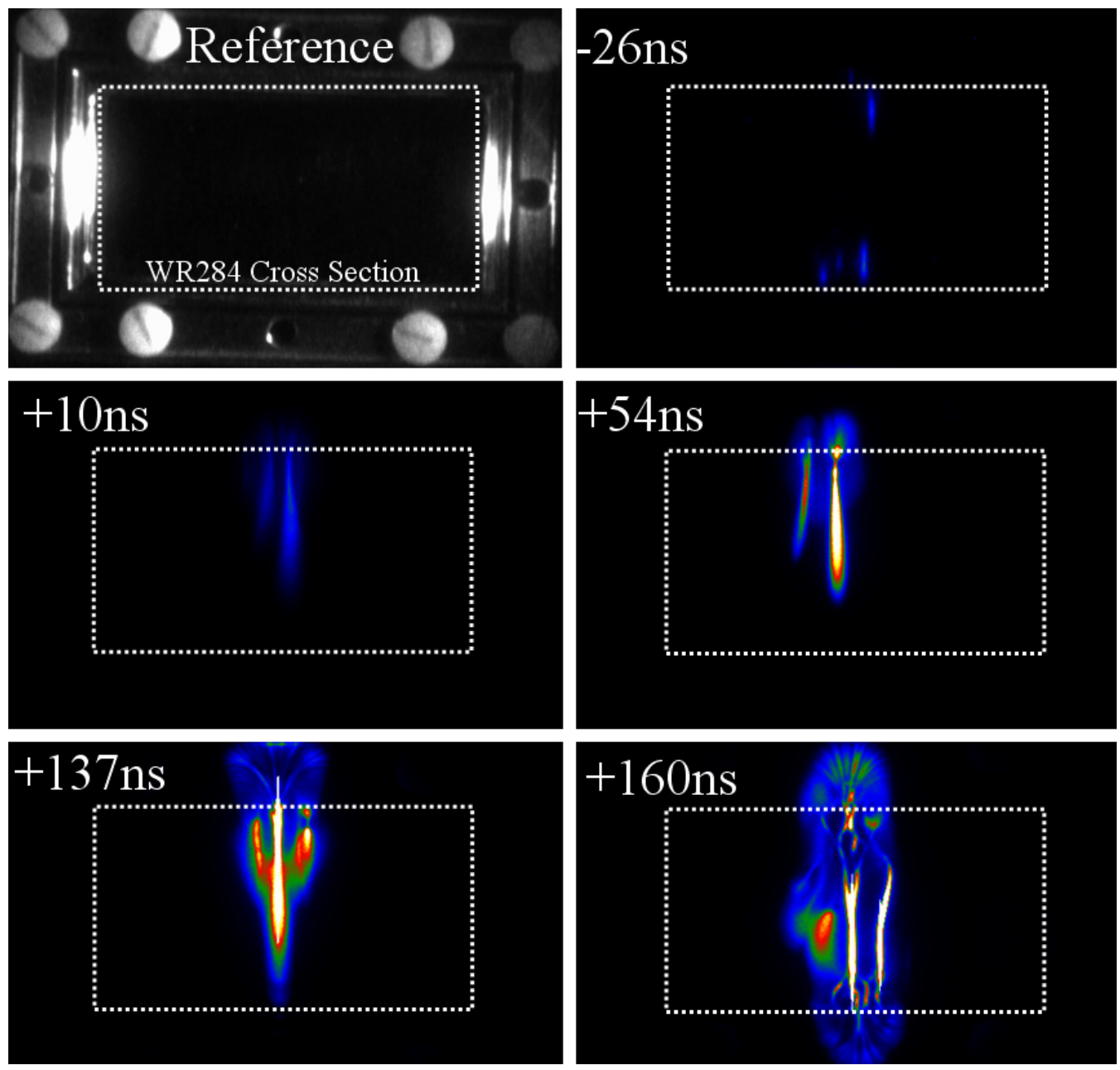

Figure 5.9: Series of pseudo-color images showing the development of a flashover with time referenced to the rise in the luminosity signal, all images taken in air at 155 torr 
As stated before, photoemission from the surface under UV radiation is assumed to play a significant role in the arc formation in an air environment. If the lack of UV production in a nitrogen environment is a significant factor in the arc formation path, then external illumination of the surface in a nitrogen environment should yield a significant change in the arc path for HPM excitation as it has for the unipolar case $[1,58]$. As a test of this reasoning a pulsed UV source is used to illuminate the surface just prior to and during the application of the HPM pulse. As the UV pulse is shifted in time closer to the flashover event the liftoff becomes less pronounced, see Figure 5.10. When the UV is applied concurrent to the flashover the arc forms as it would in an air environment. The same behavior was observed in the unipolar case confirming the role of UV for the geometry of arc formation in air and nitrogen [58]. 

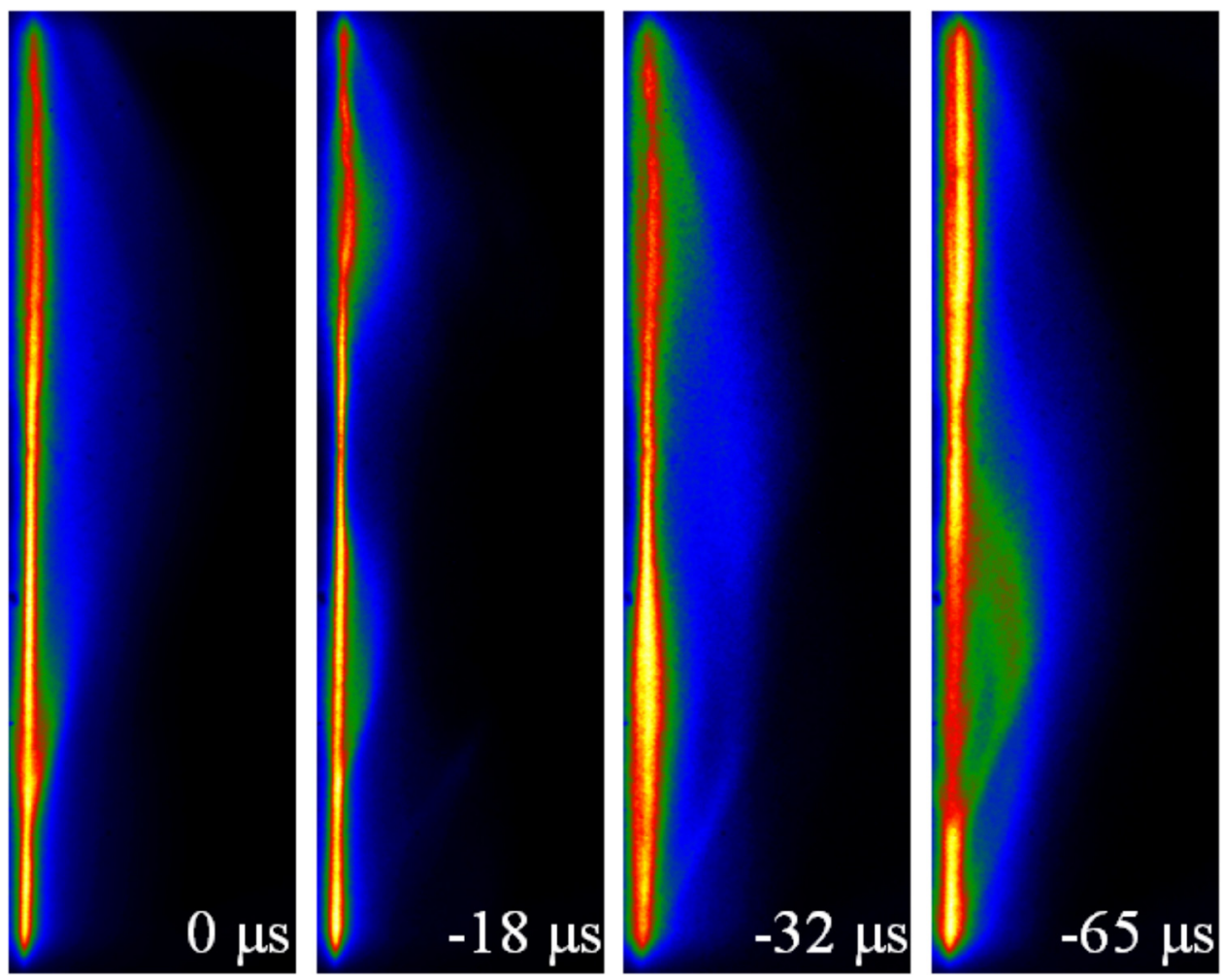

Figure 5.10: Side-on pseudo color intensity plot of main breakdown in nitrogen under HPM excitation. Time indicates delay from UV illumination of the surface to application of HPM excitation

Previous experiments with CW UV illumination of a surface during unipolar flashover have shown a decrease of up to $30 \%$ in the DC holdoff voltage compared to no illumination. A decrease in holdoff voltage will likely correspond to a lowering of the delay time at a given pulsed electric field amplitude. It was recorded that UV illumination of the surface decreased the delay time of the HPM surface flashover by up to $20 \%$ in the 125 to 190 torr range at $14.8 \mathrm{kV} / \mathrm{cm}$ field amplitude. Further analysis is necessary to address the effect UV illumination has on the statistical portion of the delay time. 


\subsection{Spectroscopy}

Optical emission spectra collected from the flashover discharge can yield important information about the processes involved in the arc development. Emission spectra are collected for both unipolar and HPM excitation. The unipolar spectra are collected with a 600 lines $/ \mathrm{mm}$ grating with a blaze of $400 \mathrm{~nm}$ and a center wavelength of $350 \mathrm{~nm}$ and is shown for both nitrogen and air environments in Figure 5.11 and Figure 5.12. There are a large number of lines, some of which have been identified, as seen in Figure 5.13. These metal lines, attributed to the brass electrodes, are prominent near the electrodes and not in the center of the gap. Comparing the spectra gathered in nitrogen and air reveals the absence of the $\mathrm{H}_{\alpha}$, at $660 \mathrm{~ns}$, in nitrogen. There is also reduced UV content produced by the arc in nitrogen.

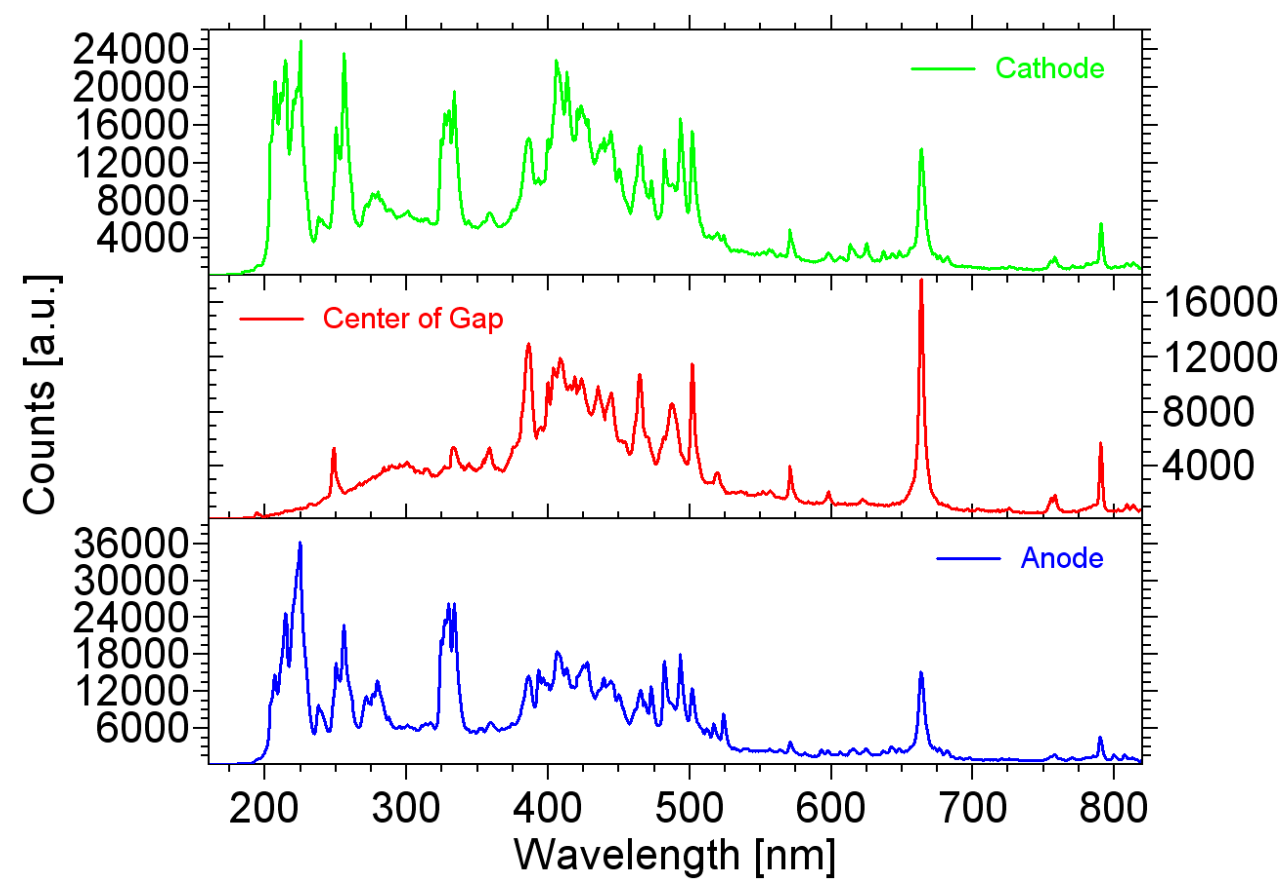

Figure 5.11: Optical emission spectra collected in air at 680 torr with unipolar excitation (top, bottom: close to electrodes, center: in between). Note the $\mathrm{H}_{\alpha}$ line at $656 \mathrm{~nm}$ 


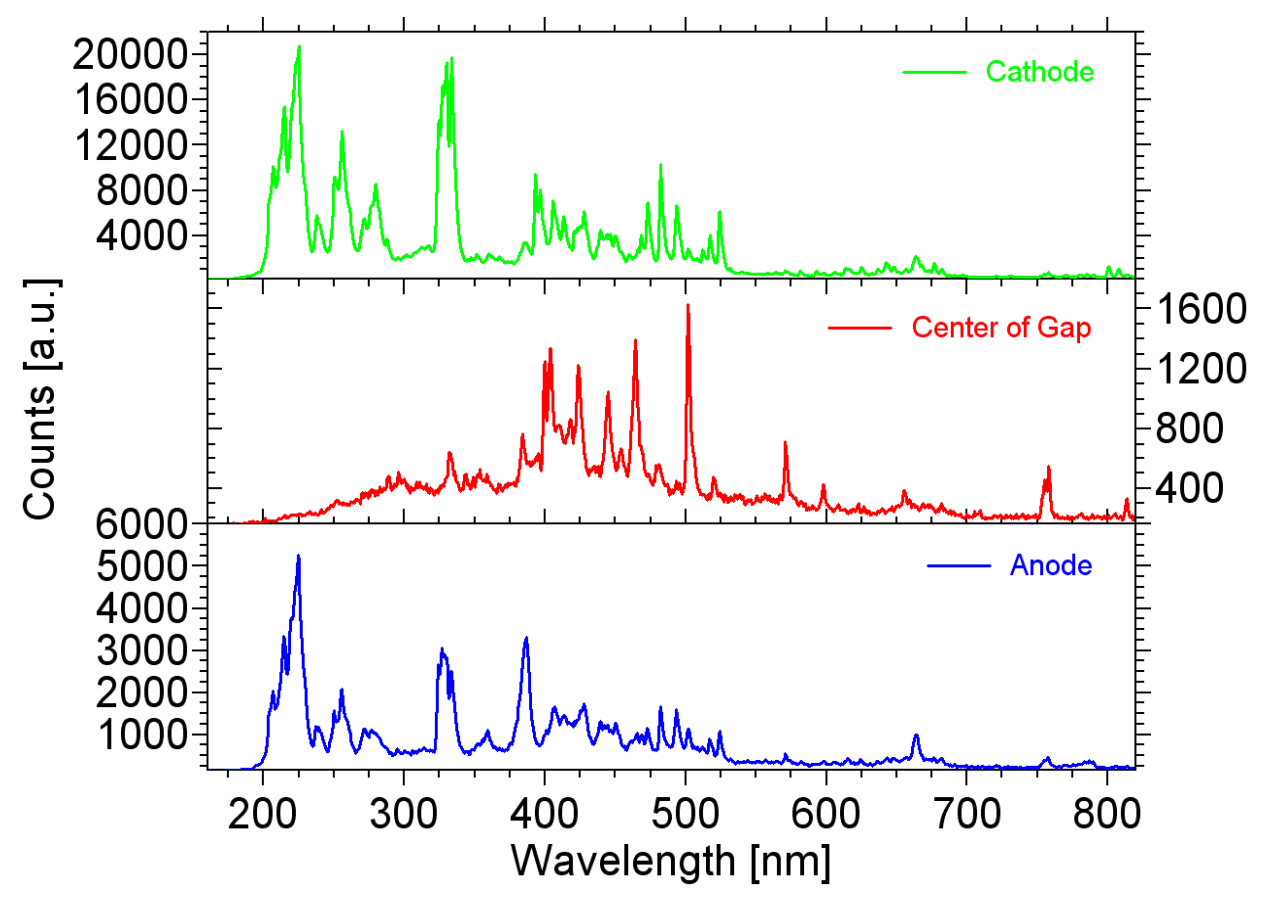

Figure 5.12: Optical emission spectra collected in nitrogen at 680 torr with unipolar excitation (top, bottom: close to electrodes, center: in between)

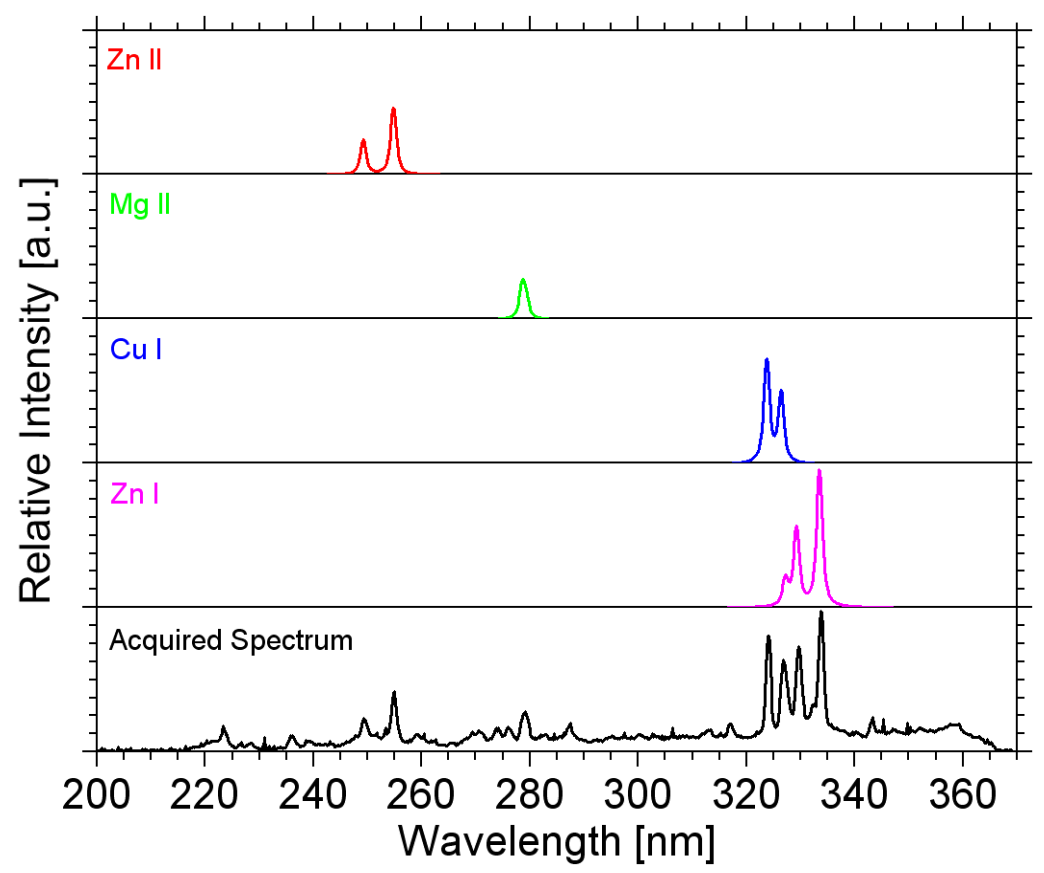

Figure 5.13: Identification of dominant peak for unipolar excitation 
Comparing the spectra in air and nitrogen environments under HPM excitation reveals that they are very similar in the higher wavelength ranges $(\lambda>300 \mathrm{~nm})$ showing the same dominant peaks, see Figure 5.14 and Figure 5.15. The HPM discharge primarily excites and ionizes the abundant gas molecules, such as molecular nitrogen. A program has been developed by Butcher, Edmiston, Morales, and the author to calculate the emission spectra for molecular nitrogen [15]. The spectral lines are identified as the $C^{3} \Pi_{u}$ - $\mathrm{B}^{3} \Pi_{\mathrm{g}}$ transitions for nitrogen, as can be seen in Figure 5.16, which show strong band heads in the HPM spectra at $315.8 \mathrm{~nm}, 337 \mathrm{~nm}, 357.6 \mathrm{~nm}$, and $380 \mathrm{~nm}$. The $\mathrm{N}_{2}$ vibrational temperature is estimated to be $4000 \mathrm{~K} \pm 500 \mathrm{~K}$ based on comparison of the observed spectra with calculated ones.

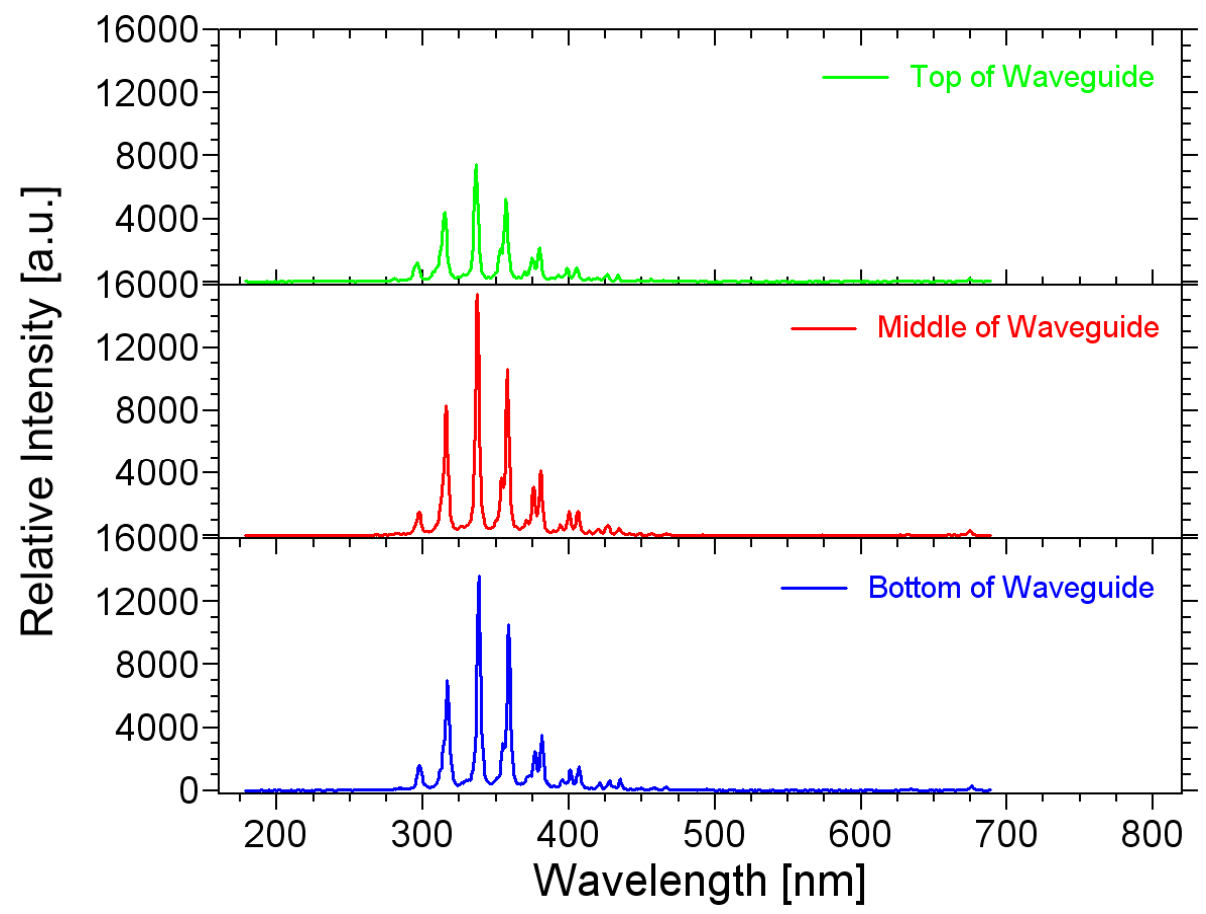

Figure 5.14: Optical emission spectra collected in air at 155 torr with HPM excitation 


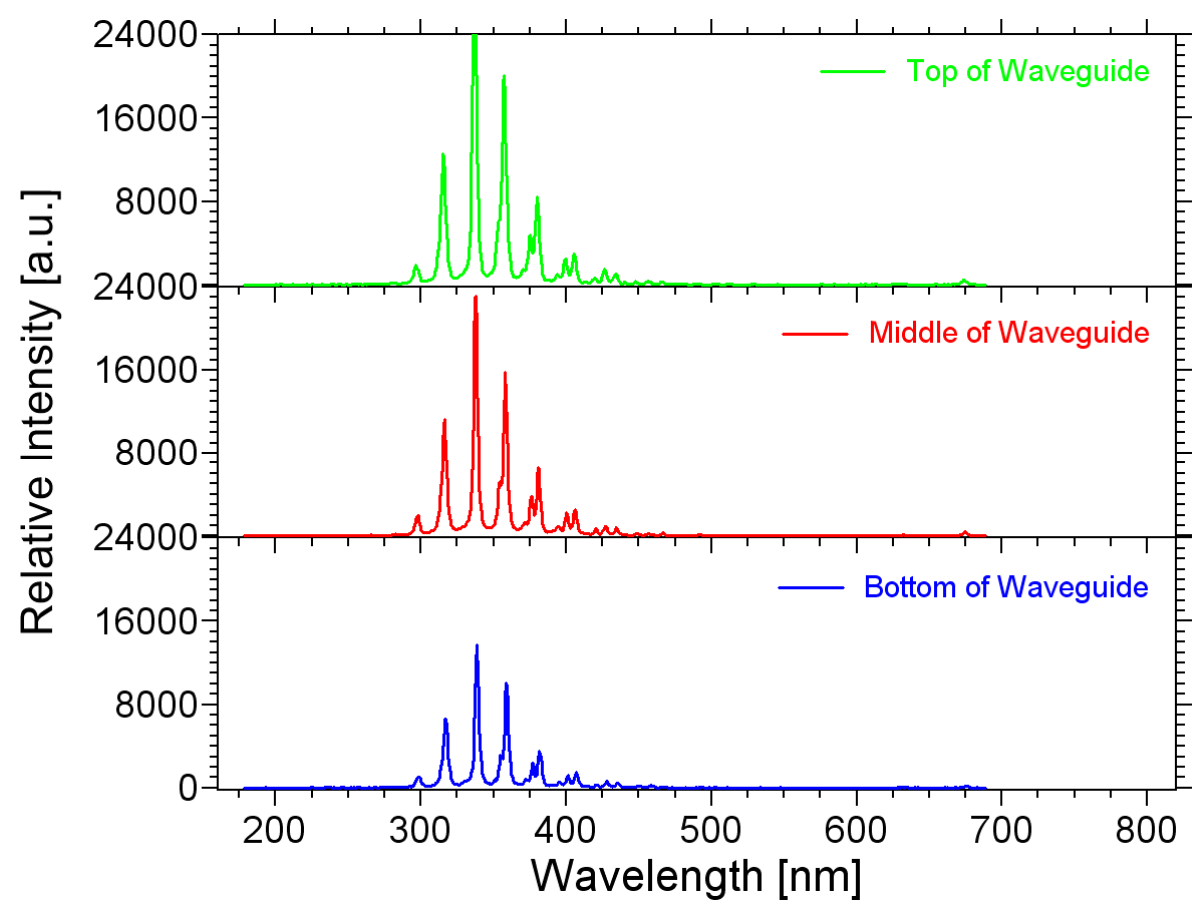

Figure 5.15: Optical emission spectra collected in nitrogen at 155 torr with HPM excitation

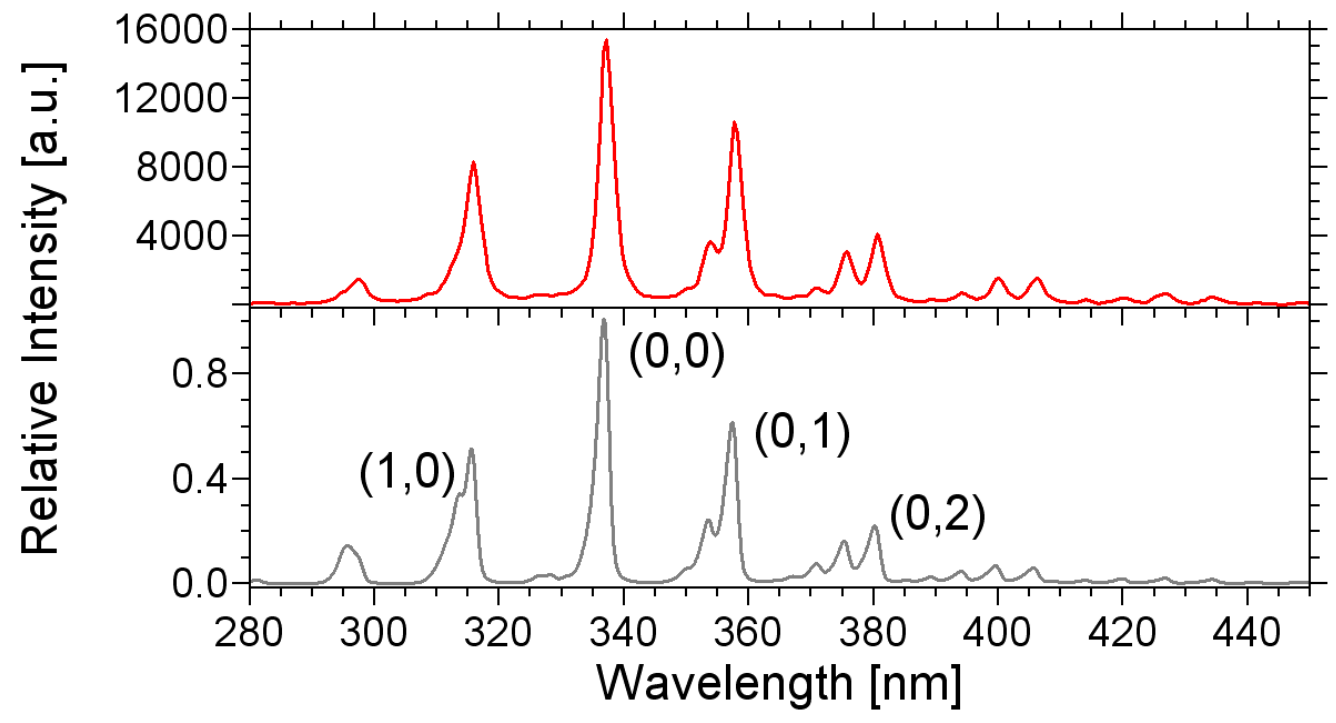

Figure 5.16: Comparison of optical emission spectra collected at 155 torr with HPM excitation, $\sim 40$ ns after arc initiation (top, red), with $\mathrm{N}_{2}$ calculated spectra shown (bottom, grey) at a vibrational temperature of $4000 \mathrm{~K} \pm 500 \mathrm{~K}$, rotational temperature is set to room temperature, the vibrational transitions responsible for the various band heads are labeled [15] 
The differences in the nitrogen and air environments are obvious at UV wavelengths, cf. Figure 5.17. There is more UV production from the HPM flashover in air than in nitrogen. It should be noted that UV emission below the apparatus detection limit of $180 \mathrm{~nm}$ is also more than likely involved in the discharge formation. This holds especially true considering that the photoemission from dielectric surfaces is highly dependent on photon energy, with higher yields at higher energies [21 - 23]. The quantum yield for a given surface, $\gamma_{v}$, is generally $\sim 10^{-3}$, in the near-UV region and $\sim 10^{-2}-10^{-1}$ in the far-UV region.

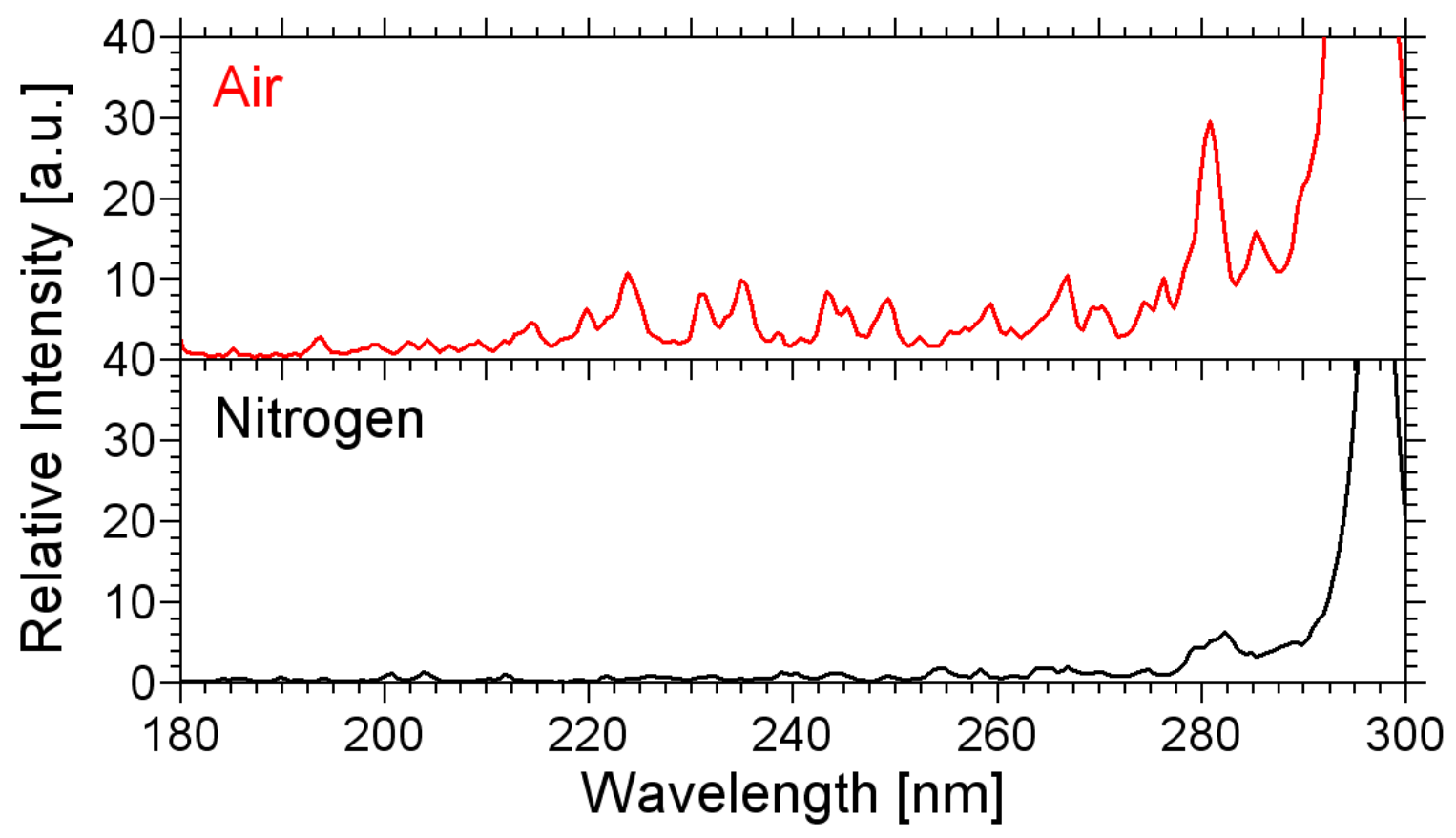

Figure 5.17: Optical emission spectra collected at 155 torr with HPM excitation, $\sim 40 \mathrm{~ns}$ after arc initiation. Scale is adjusted relative to the dominant peak at $400 \mathrm{~nm}$ for highlighting the increased UV content in air environment

Comparing unipolar to HPM surface flashover, several differences become obvious. The larger number of peaks in the unipolar spectra, as compared to HPM excitation, is 
most likely due to the difference in plasma temperature between the two types of arcs. As a discharge forms under HPM excitation it begins to reflect the microwave power, leading to limited heating of the developing plasma. The unipolar arc does not have this limitation and therefore yields higher electron densities and temperatures in the gap. When the discharge is fully formed, the plasma in the unipolar case is expected to have higher energy sufficient to dissociate the water molecules in the air and to excite/ionize the various molecules and elements present in the environment. This explains the presence of the hydrogen alpha line in the unipolar spectra; in the HPM case the plasma temperature must be lower as the water molecules are not significantly dissociated. Finally, there is a presence of metal lines from the electrodes in the unipolar spectra. These lines do not exist in the HPM spectra due to the lack of electrodes.

It is possible that the unipolar flashover produces spectra during early arc formation similar to the HPM flashover spectra. However, the early phase of unipolar flashover is difficult to capture with single shot imaging spectroscopy due to the fast arc development (1-4 ns) as well as the rather large standard deviation of the flashover delay time (several 10 's to 100 's of ns). Utilizing a streak camera in place of the single frame ICCD should provide more information in the future.

\subsection{Humidity Effects}

For unipolar excitation it has been shown previously that humidity lowers the flashover field amplitude [1, 59]. This behavior holds true for both nitrogen and air environments as seen in Figure 5.18. A direct comparison of the effects of humidity given 
DC unipolar and HPM excitation is difficult as the flashover potential is set for HPM excitation and HPM excitation can only be pulsed, and not CW. However, a correlation can be made between a decreased flashover delay time and a lowering of the flashover voltage. It is expected that an increase in humidity under HPM excitation will correspond to a decrease in the flashover delay time. Figure 5.19 shows that the delay times do in fact decrease with increased humidity for HPM excitation.

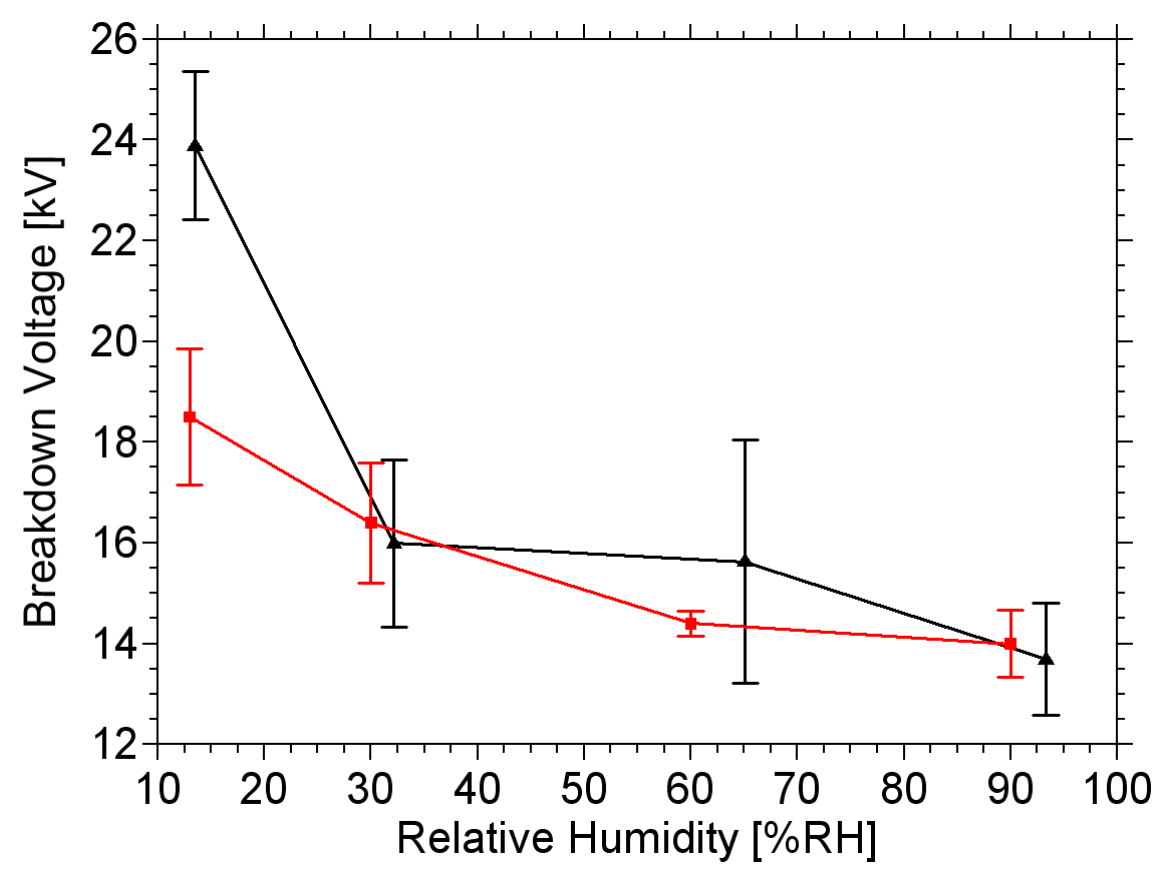

Figure 5.18: Breakdown voltage vs. relative humidity in air (red circles), and nitrogen (black triangles) for unipolar excitation [47] 


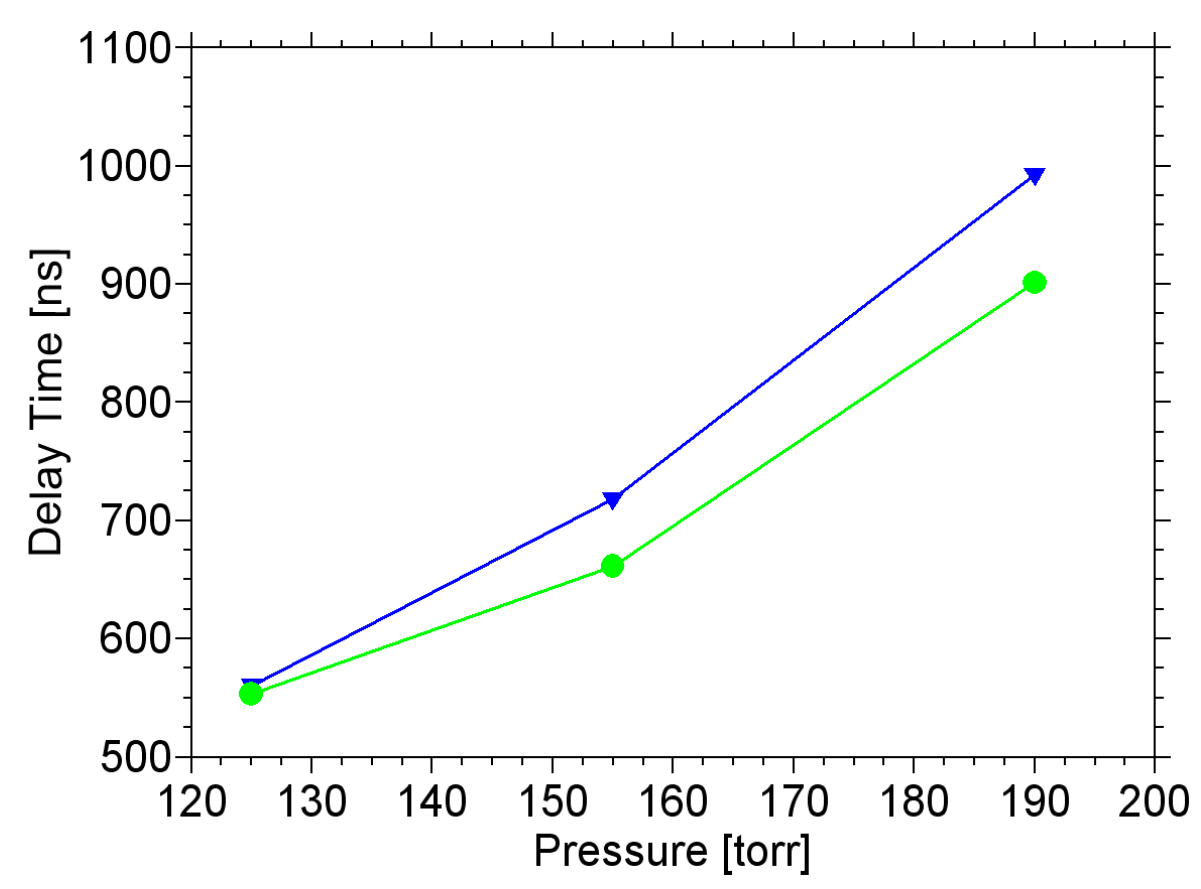

Figure 5.19: Pressure vs. delay time in air at low humidity $(<15 \% \mathrm{RH})$, triangles, and high humidity $(>85 \% \mathrm{RH})$, circles, for HPM excitation of $3 \mathrm{MW}(\sim 13.5 \mathrm{kV} / \mathrm{cm})$

It is believed that the humidity affects the breakdown electric field amplitude by creating a thin layer of water on the surface (adsorption), which is more easily ionized [47]. The higher the humidity the thicker the layer on the surface, and the easier it is for the impacting electrons to produce secondary electrons. Impurities in the water may also play a role in affecting the breakdown voltage; however, the water used was purified and de-ionized in order to limit contamination.

\subsection{Monte Carlo Simulation}

A Monte Carlo type simulation code was developed to further isolate the roles of various processes that dominate the surface flashover. As there already exist Monte Carlo type simulations written for surface flashover at atmospheric pressures $[3,56]$, the 
present simulation focuses on HPM excitation. In order to limit the calculations the only area simulated is the high field region near the center of the WR284 waveguide, cf. Figure 5.20.

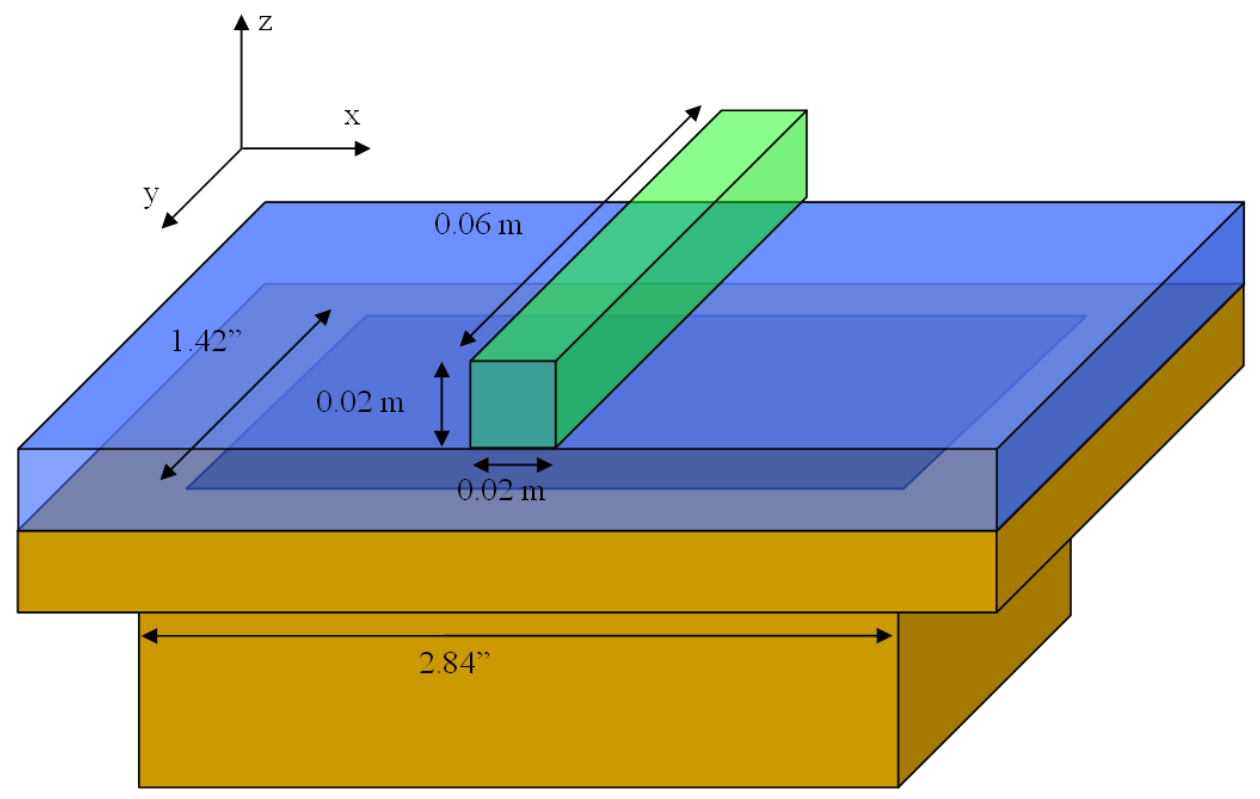

Figure 5.20: Drawing of WR284 exit flange (brown), polycarbonate sample (blue), and the simulation area (green box). The coordinate system is defined as shown

\subsubsection{Early Code}

The Monte Carlo simulation was produced in iterations. The first of these iterations was a very simple zero dimensional code that can only handle hundreds of electrons at a time, moving only left or right under a uniform alternating electrical field. These electrons experienced only the most basic collisions determined from highly idealized collision cross sections. After several rounds of improvements the code was expanded to include multiple collision cross sections, automated parameter input, automatic calculation of the electron energy distribution function, or EEDF, and a host of other 
small changes to improve performance and accuracy. The code was also reformatted to operate on a multi-thread protocol called MPI, or Message Passing Interface. This allowed the program to be executed on a Beowulf cluster located in the Physics Department at Texas Tech University. This cluster, called Gamera, consists of 16 computer containing AMD 64-bit processors and 1GB RAM each connected by a dedicated T1 network running under the MPI standard protocol.

The results produced by the zero dimensional code can be seen in Figure 5.21. For comparison another program was acquired that calculates the average steady state EEDF for a given electron density, gas composition, and applied electric field. The program, called the EEDF calculator, was created by Dr. Napartovich and produces the EEDF by solving the Boltzmann equations [49]. The output of the EEDF program is shown in Figure 5.21 and is comparable to the simulation results in Figure 5.22. A key difference in the distributions lies near $0 \mathrm{eV}$. The Monte Carlo simulation shows a spike near $0 \mathrm{eV}$ which is not present in the EEDF calculator's results. This can be attributed to the zero dimensionality of the program. The full velocity, or energy, of a given electron is subject to the alternating field at all times. The electron has no way of "storing" energy by, for instance, colliding with a molecule, getting deflected, and transferring some of its velocity component into a direction not influenced by the electric field. This velocity/energy is then "stored" in that it cannot be reduced except by another collision. At this point the zero dimensional code has reached its limits and a full $3 \mathrm{D}$ version of the code, capable of running on the MPI standard, was constructed. 


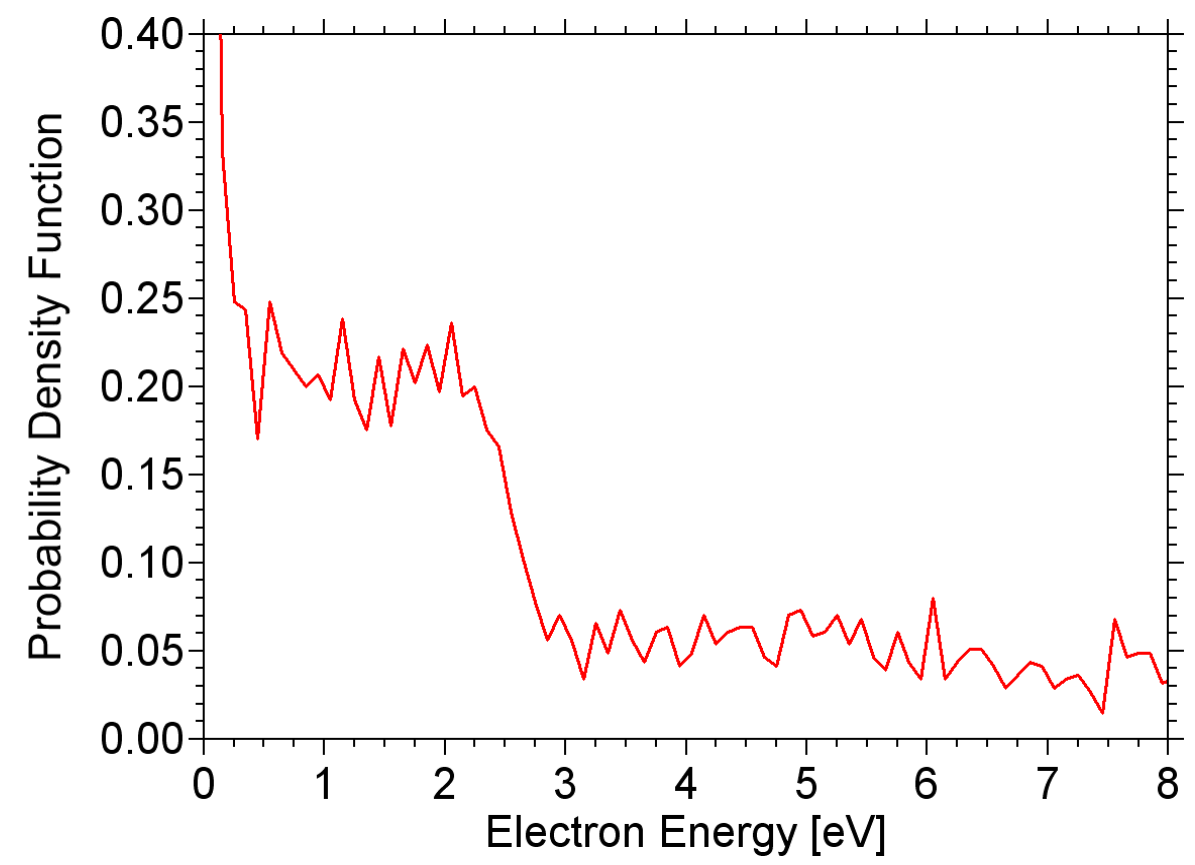

Figure 5.21: Electron energy distribution generated by the 1D simulation in pure nitrogen, 1000 initial electrons, $5.75 \mathrm{kV} / \mathrm{cm}$ electric field with a frequency of $2.85 \mathrm{GHz}$

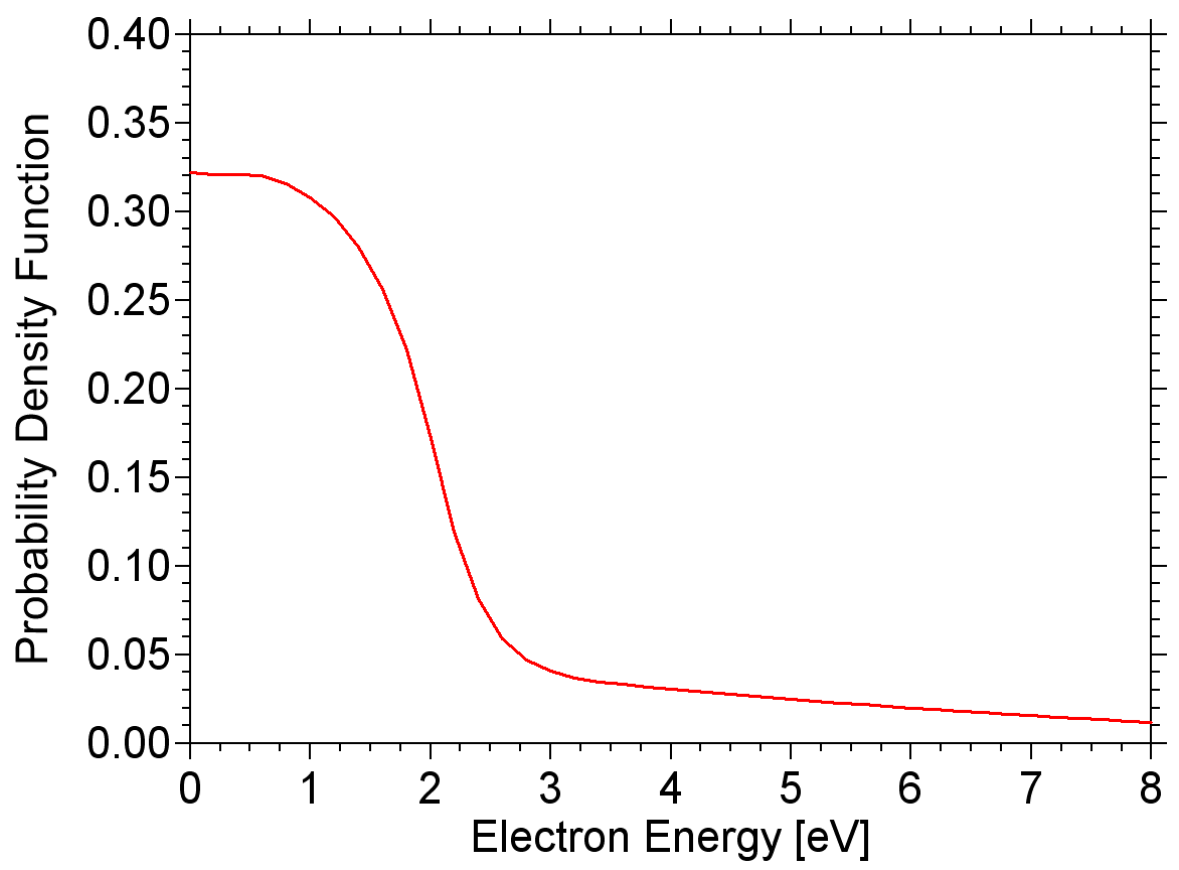

Figure 5.22: Electron energy distribution generated by the EEDF calculator in pure nitrogen, $5.75 \mathrm{kV} / \mathrm{cm}$ electric field with a frequency of $2.85 \mathrm{GHz}$ 


\subsubsection{Sensitivity Testing}

Before beginning extensive usage of the 3D version of the program some tests were performed to determine the sensitivity of the program itself to certain variables. This also aided in the debugging process by creating extreme operating conditions while analyzing the output for anomalies. The first tests involved altering the dominant collision cross sections, such as for elastic collisions. As Figure 5.23 shows, the collision cross sections have a profound effect on the simulation. By multiplying the cross section by 0.5 and 2 , large shifts in the time necessary for exponential electron growth become evident. This is important as the cross sections used will need to be as accurate and up to date as possible.

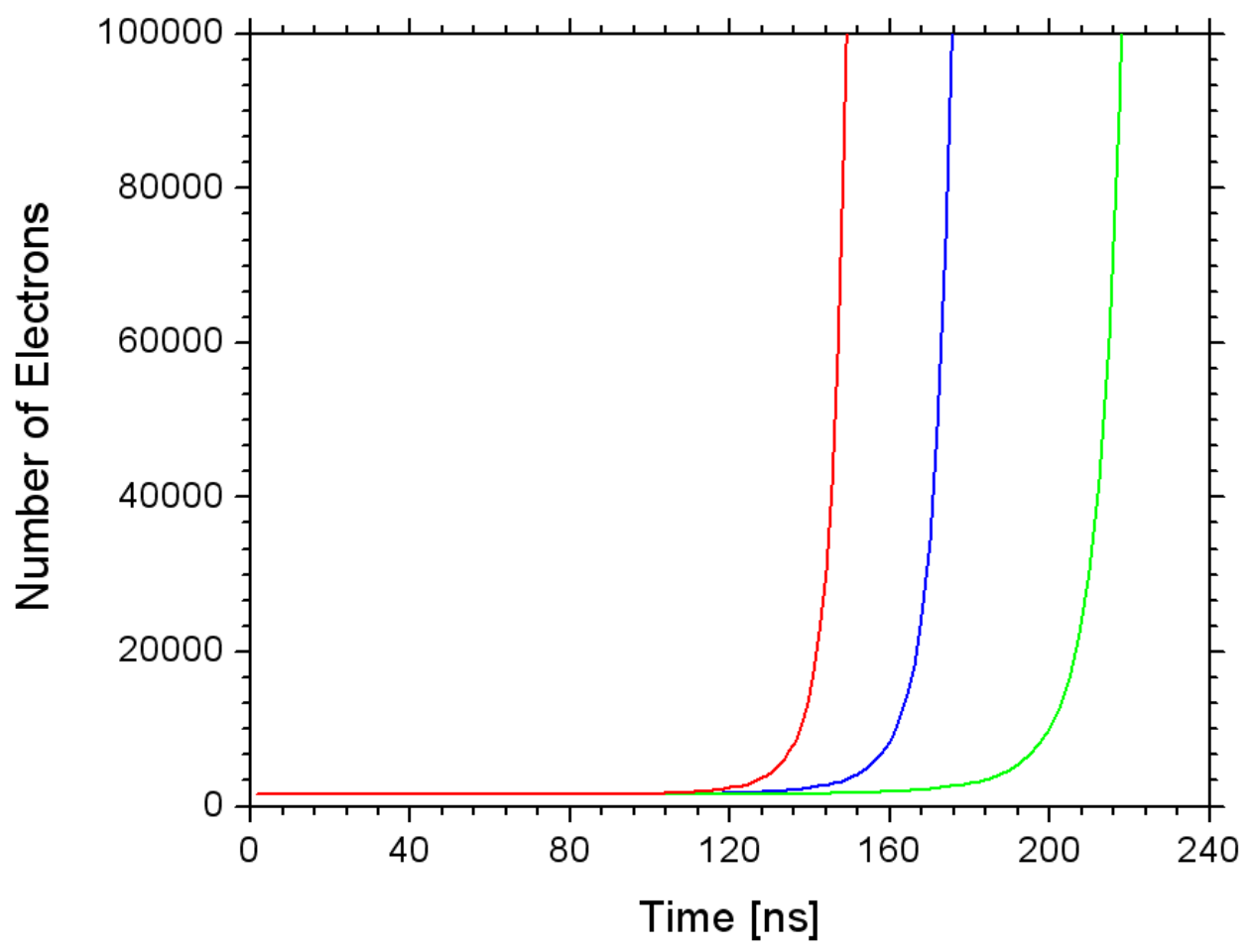

Figure 5.23: Total electron count verses simulation time in a nitrogen environment with the real elastic cross section (blue), half the elastic cross section (red) and double the elastic cross section (green) 
The second test involves altering the number of initial seed electrons. In air there are approximately 1000 ion pairs $/ \mathrm{cm}^{3}$ due to cosmic radiation, at least some of which are detached in the high field region, therefore the initial electron concentration must be less than 100 electrons $/ \mathrm{cm}^{3}[60]$. As the simulation requires the initial number of electrons to be divisible by the number of nodes, or computers in the array, a standard electron density of 1600 electrons, equating to 66.6 electron $/ \mathrm{cm}^{3}$, is used for air environments. As there are no know stable negative ion pairs for nitrogen, the initial electron density is chosen to be as small as possible, or 1 electron per node which yields an electron density of 0.66 electrons $/ \mathrm{cm}^{2}$. For testing purposes this value is increased by several orders of magnitude. Figure 5.24 shows that the initial electron concentration plays a less significant role in the flashover delay time than the collision cross sections. However, it still needs to be carefully considered for a given environment. 


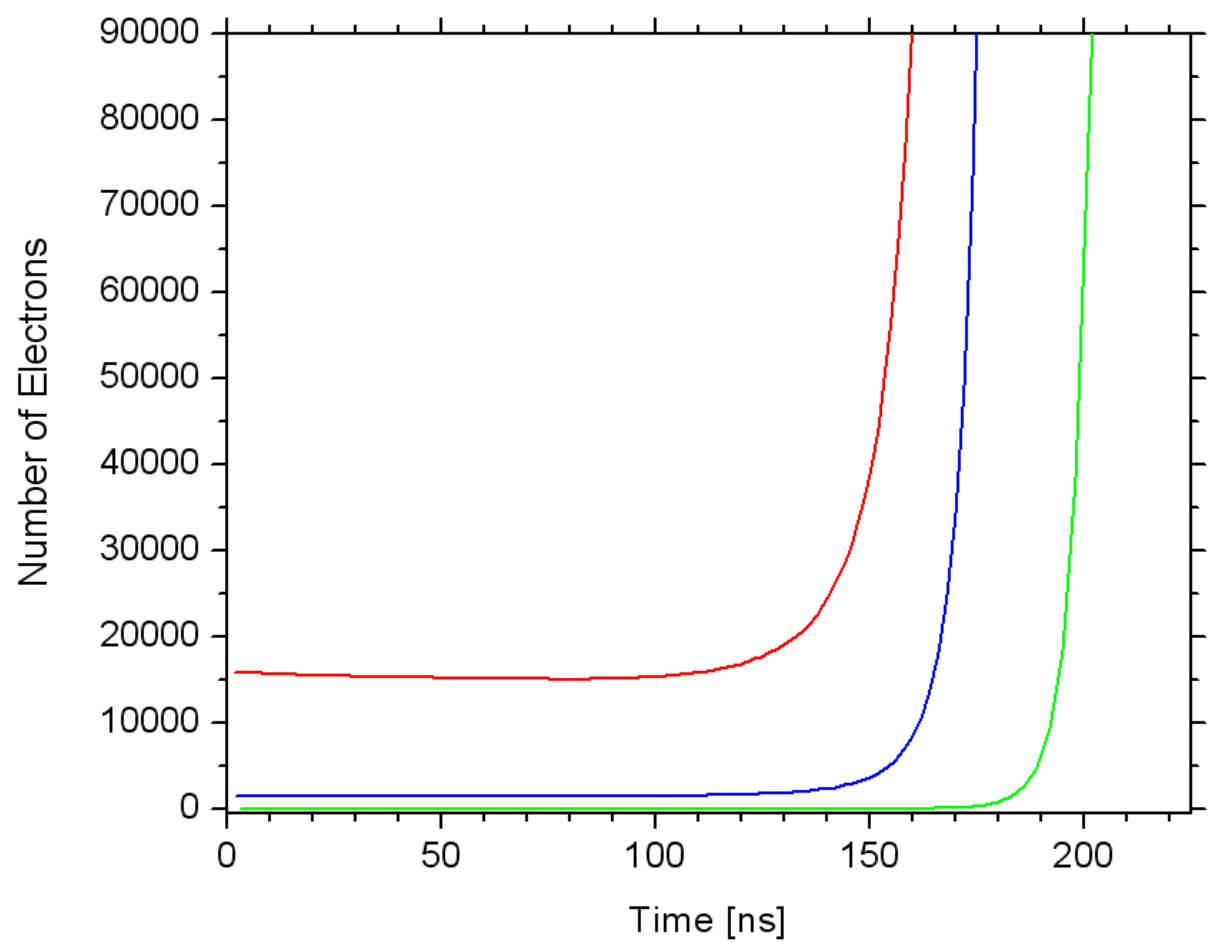

Figure 5.24: Total electron count verses simulation time in a nitrogen environment with the 16 seed electrons (green), 1600 seed electrons (blue) and 16000 seed electrons (red)

Another test preformed involved the seeding of the initial electrons within the simulation volume. The seed value of the random number generator used in the placing of the electrons in the volume is adjusted by a range of scaling factors. These simulations were run in an air environment with an ideal square pulse. As shown in Figure 5.25 below, the variation of the seeding value by several orders of magnitudes yields only small changes in the rate of electron amplification over the course of the simulation. This small change indicates that some of the statistical variations in the experimental data could be attributed to the initial electrons' spatial distribution within the volume. 


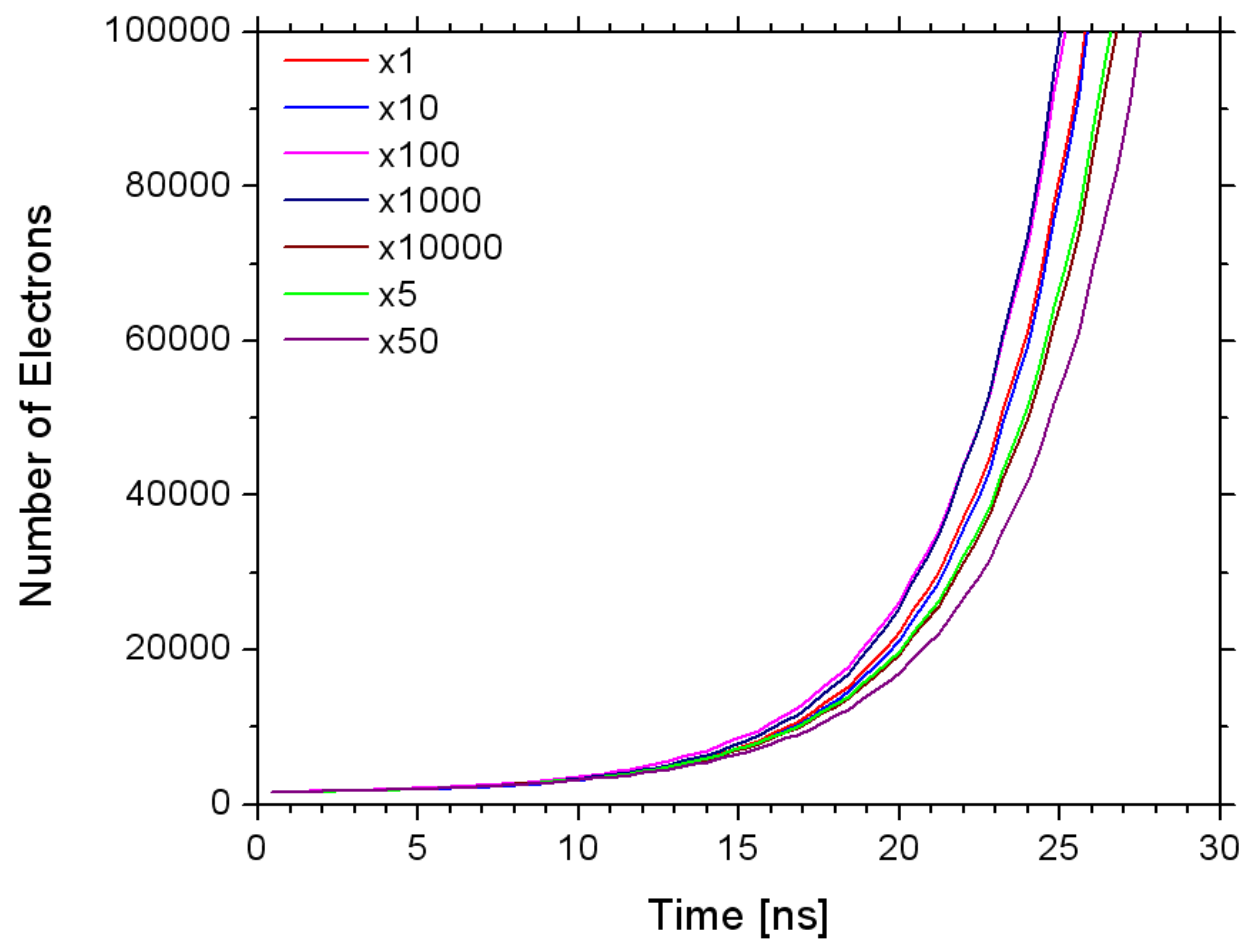

Figure 5.25: Demonstration of sensitivity of the simulation to the initial placement of electrons within the volume, each curve represents the seed value of the random number generator varied by the given factor.

The last test performed involved varying the frequency of the applied field. While all simulations run for comparison to the experimental setup are set at $2.85 \mathrm{GHz}$, the code is capable of simulating higher frequencies. For this test the frequency is varied from $1 \mathrm{GHz}$ to $100 \mathrm{GHz}$ with all other variables held constant as seen in FigXX. There is a steady increase in the flashover delay time with the increase in frequency, especially in the $30 \mathrm{GHz}$ range. Also, the nitrogen delays are almost always higher that those in an air environment, as expected. It should be noted that as the frequency increases the wavelength becomes small in comparison to the width of the simulation region. Because of this the simulation frequency should be limited to $\sim 30 \mathrm{GHz}$. 


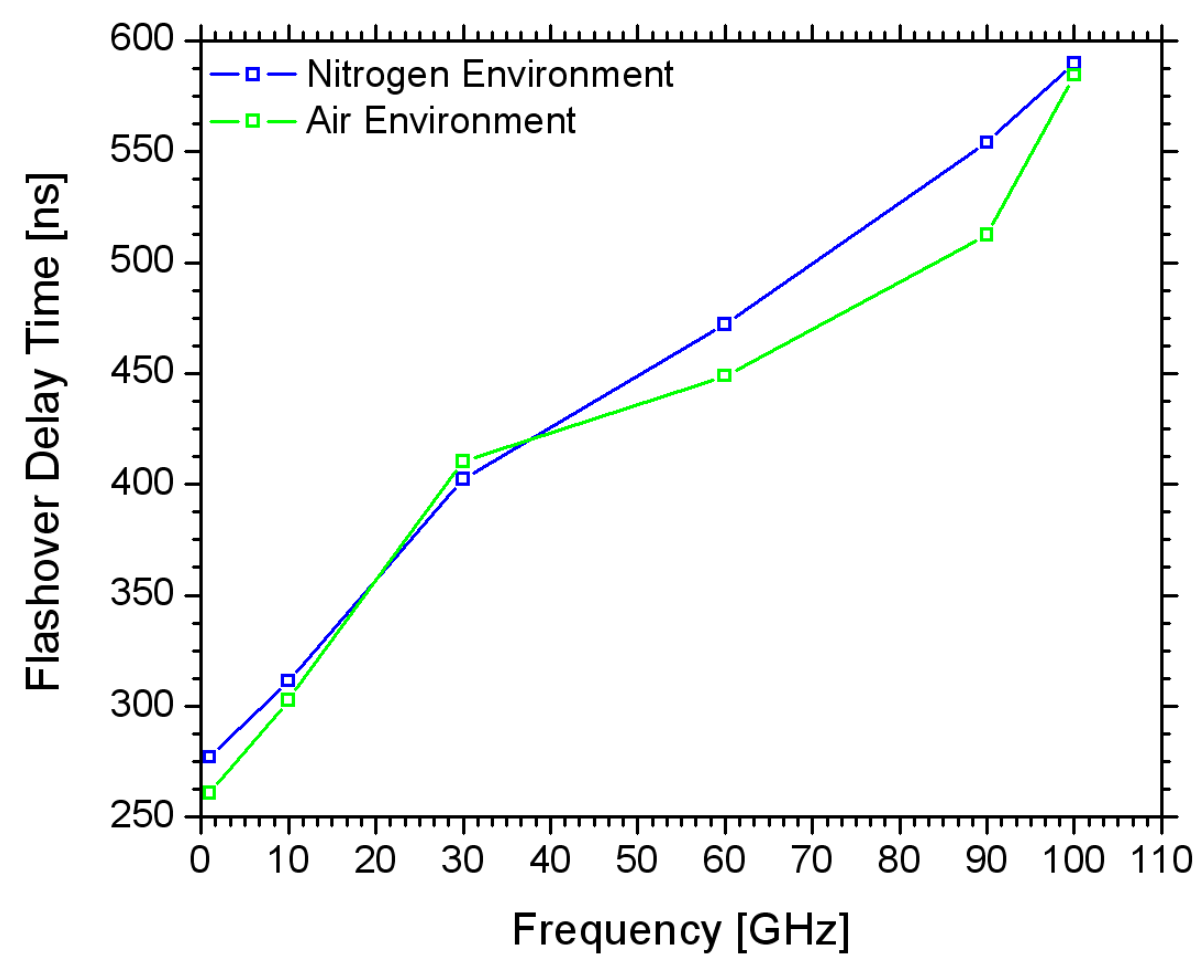

Figure 5.26: Flashover delay times in both air and nitrogen environments for varying frequencies at 100 torr under $15 \mathrm{kV} / \mathrm{cm}$ excitation

\subsubsection{Arc Formation}

An advantage of simulating the motion of the electrons is the ability to get a picture of the arc channel formation. It is reasonable to assume the arc will form where the electron clouds develop. As the simulations show, the electron clouds do not form while traversing the gap as they do in unipolar excitation. Instead the clouds stay relatively localized due to the oscillatory nature of the field and the frequent elastic collisions $(\mathrm{THz}$ collision frequency at 1 atm pressure). The clouds grow primarily by diffusion. Figure 5.27 shows several time steps from a simulation of 16 initial electrons in a nitrogen environment at 80 torr and a maximum field strength of $15 \mathrm{kV} / \mathrm{cm}$. The initial electrons remain relatively stationary while the field ramps up, similar to the source used in the 
HPM setup as shown in Figure 5.28. It is important to note that the code does not simulate the arc; it only calculates the electron motion and density due to the various collisions, such as ionizing, elastic, attachment, etc., without taking into account coulomb forces between the electrons. The effect of the background ions is also neglected as their density is very low, on the order of that produced by the electron cloud, thus producing a field of around $0.005 \mathrm{kV} / \mathrm{cm}$ in the early stages of flashover. Moreover, the electron cloud formation leaves the electrons in the vicinity of the largest ion background, hence partially neutralizing the electron space charge.

The final formation of an arc is defined by the moment when the critical plasma density is reached, see Eq. 2.7. At this density the plasma reflects the incident microwaves, which would correspond to an increase in the reflected power. As stated previously, the delay time measured with the experimental setup is from application of the pulse to the rise in reflected power. Since the MC code is focused on the early developmental stages of flashover, the electron density will be extrapolated to breakdown values as described in Chapter 5.4.4. 


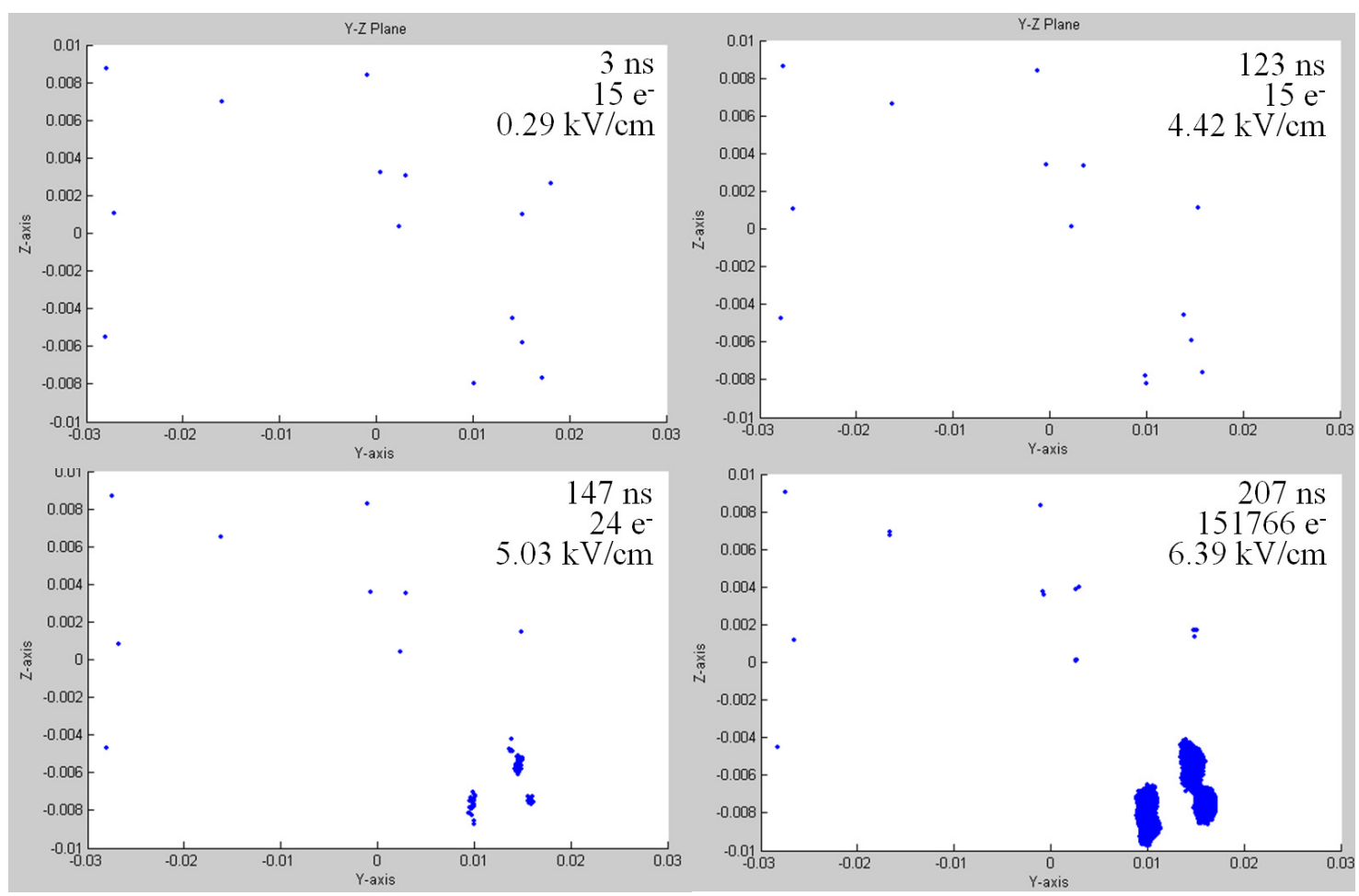

Figure 5.27: Electron cloud formation in a nitrogen environment with 16 seed electrons

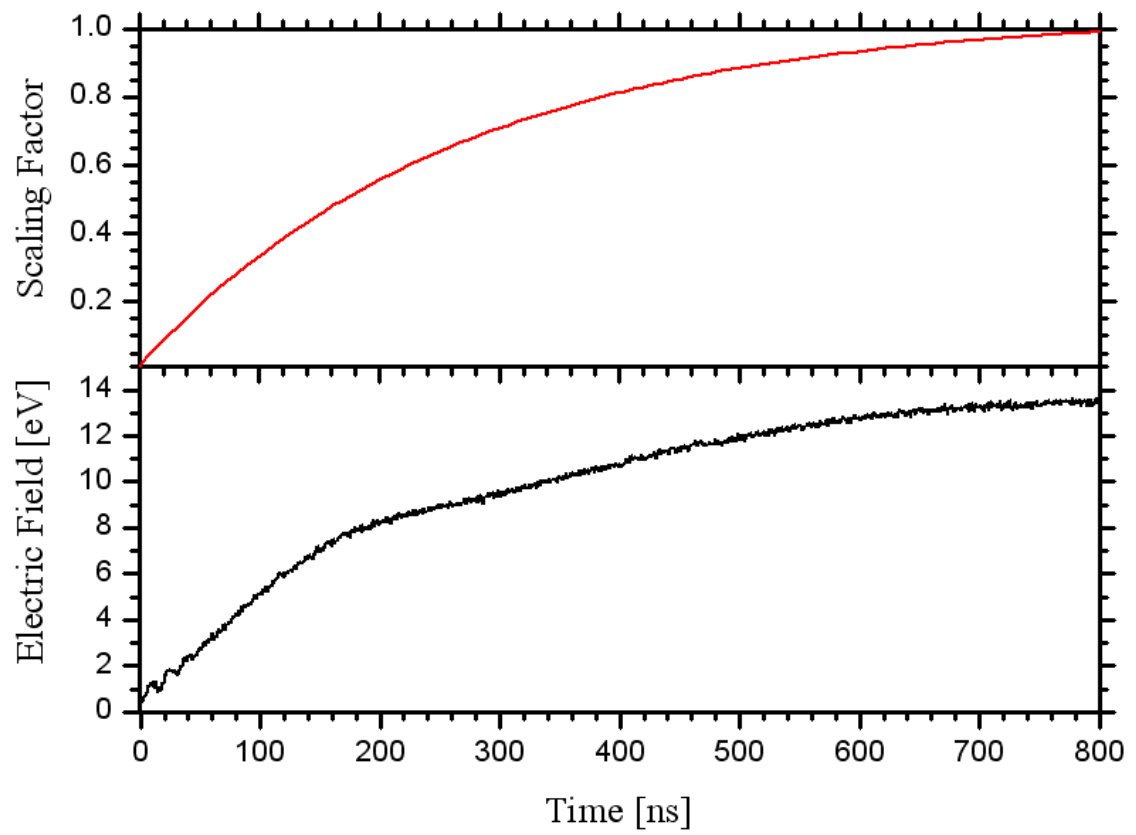

Figure 5.28: Comparison of the electric field scaling function (top, red) and the actual ramp up (bottom, black) associated with the HPM source 
Once the field reaches a sufficient level the electrons gain enough energy to begin ionizing the surrounding molecules and the electron cloud formation begins. Due to the lack of electronegative oxygen molecules, the only loss process is diffusion of the electrons away from the simulation area. As a result, any electrons at or above the surface will eventually begin to ionize the nitrogen, thus forming electron clouds, and eventually plasma channels, at and above the surface. This is very similar to arc formation tendencies observed with the HPM setup, as shown in Figure 5.9.

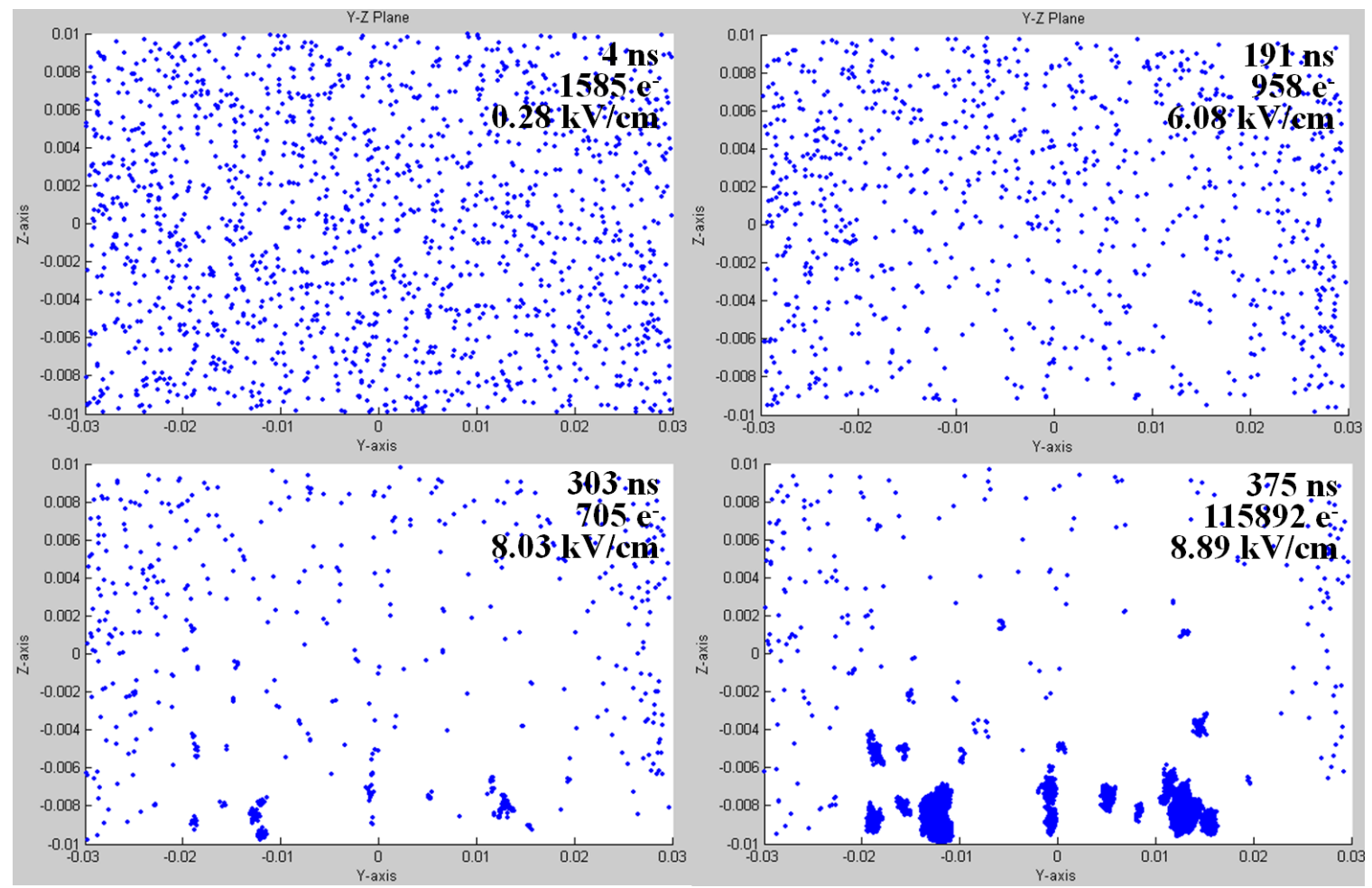

Figure 5.29: Electron cloud formation in an air environment with 1600 seed electrons

An arc in air is simulated with 1600 initial electrons, shown in Figure 5.29. This simulation is different because of the addition of the oxygen cross sections, most notably the attachment cross section. The threshold energy for attachment, $\sim 4.4 \mathrm{eV}$, is much 
lower than the ionization thresholds for nitrogen, $\sim 16.5 \mathrm{eV}$, and oxygen, $\sim 12.5 \mathrm{eV}$. As a result the initial electrons are lost to attachment as they are accelerated by the field. This yields a loss of electrons in the high field region. This contradicts the observed arc channel formation in air, because the arc would seem to form away from the surface as it is the location of the highest field. A possible explanation is the exclusion of a photoelectric effect in which the surface is irradiated with photons caused by deexcitation of the oxygen and nitrogen molecules. This effect is believed to be an important factor in the differences between nitrogen and air flashover events, and will be included in future iteration of the code. A surface charge buildup due to impacting electron capture is another possible explanation. However, given the small number of electrons impacting the surface, usually less than 1000, the acquired surface charge induced field is many orders of magnitude smaller than the applied field, on the order of $10^{-7} \mathrm{kV} / \mathrm{cm}$.

\subsubsection{Flashover Delay}

Another value that can be determined from the simulation is breakdown delay time. That is the amount of time from the application of the pulse to the flashover of the surface. As stated previously, the simulation does not simulate breakdown directly, only the motion of the electrons during the early stages of arc formation. In order to determine the predicted delay time the following procedure is used. The simulation is run until a minimum of 100,000 electrons exist within the simulation region. The number of electrons vs. time is plotted and a curve fit is applied as seen in Figure 5.30. A flashover 
criterion is calculated, based on the critical plasma density equation in section 2.6 and the approximate area of the arc region, to be $2.4 * 10^{11}$ total electrons for $2.85 \mathrm{GHz}$. The value of the curve fit at the flashover criterion is said to be the flashover delay time, $\tau$.

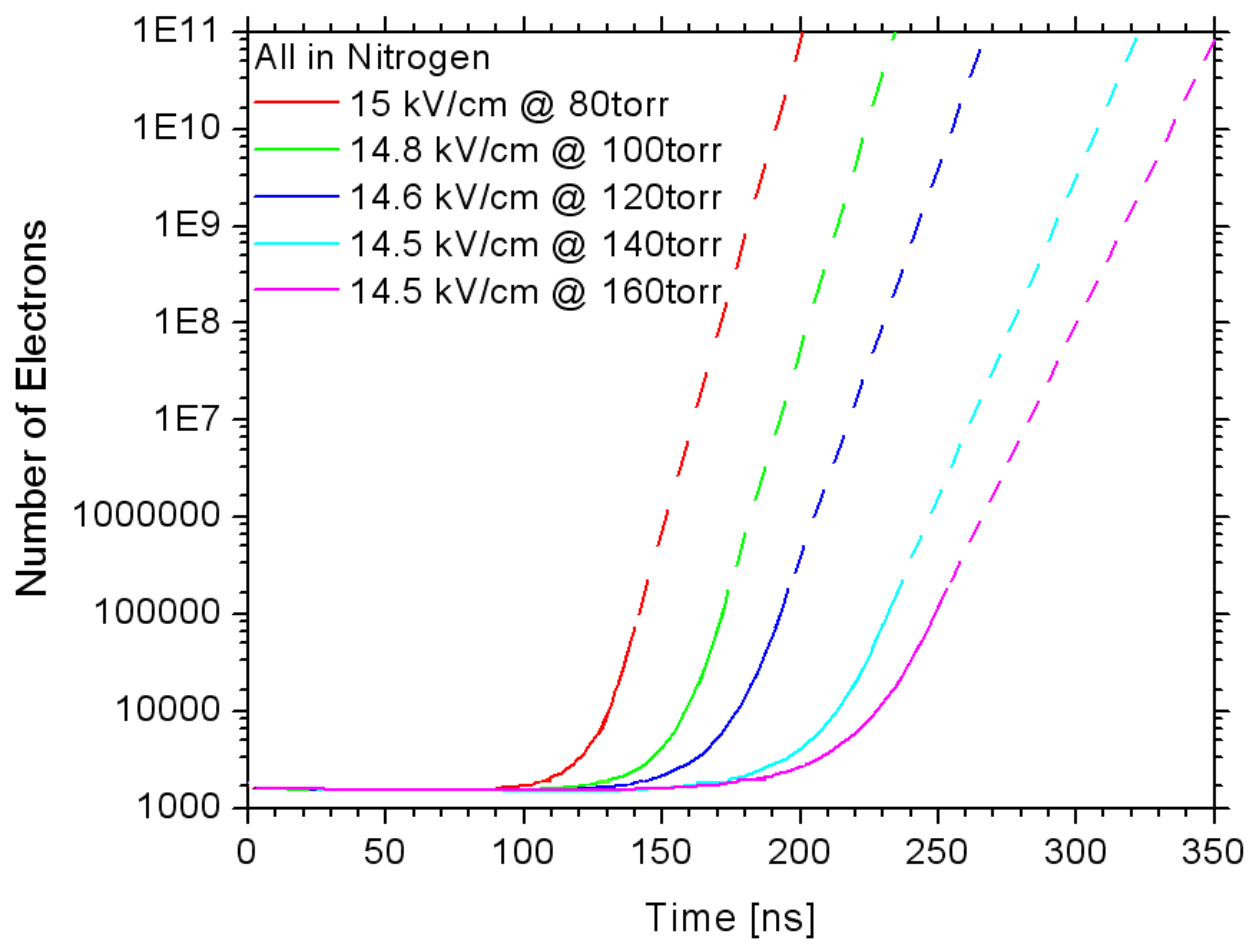

Figure 5.30: Demonstration of method used to extrapolate flashover delay times, solid portion of the curves are calculated at various pressures and fields, dashed portion of the lines are curve fits

Calculations using an ideal square pulse, as seen in Figure 5.31, show that for a given pressure the delay time decreases with the increased strength of the applied electric field for both air and nitrogen environments as expected. Additionally, flashovers in nitrogen tend to have longer delay times than those in air. This is in agreement with previous experimental findings from Edmiston [15]. 


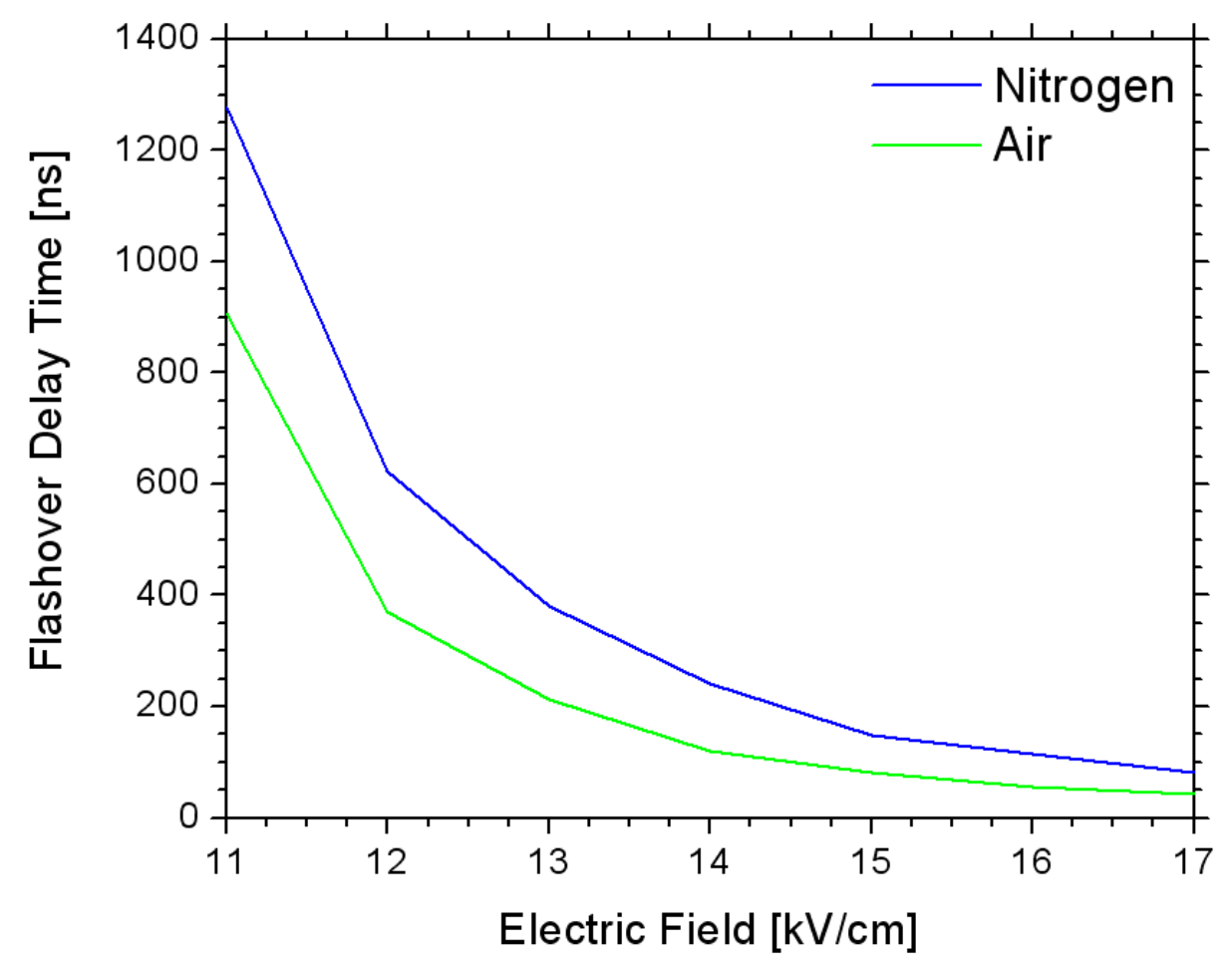

Figure 5.31: Calculated flashover delay time versus electric field in air and nitrogen at 200 torr excited with an ideal square pulse

Gould and Roberts [44] developed a theory for microwave volume breakdown that can be applied to estimate the relationship of several breakdown parameters including delay time, microwave frequency, pulse amplitude and pressure. From this theory a pulse breakdown curve equating the normalized effective field to the breakdown delay time multiplied by the pressure is derived. This relationship can now be applied to surface flashover under HPM excitation. Figure 5.32 shows the curve derived by Gould and Roberts for a volume breakdown along with data collected with the HPM flashover experiment in an air environment. 


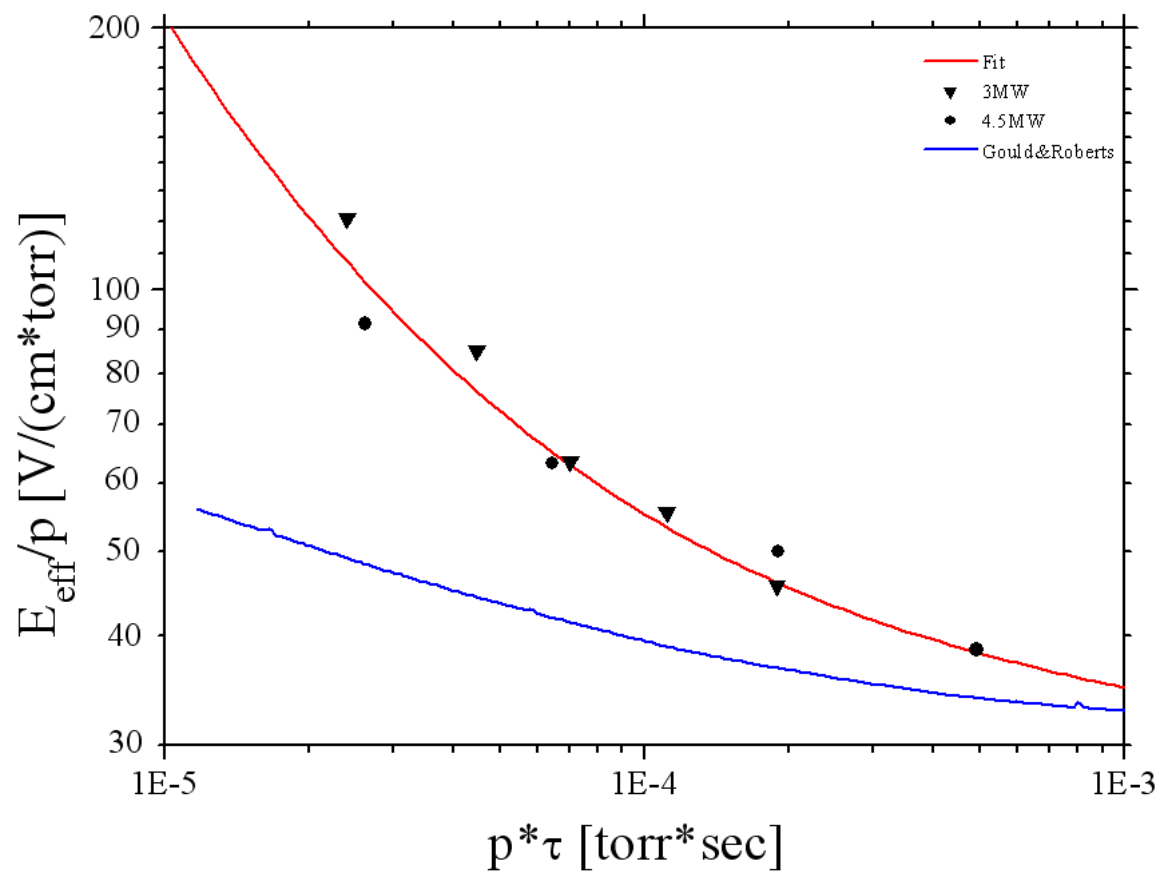

Figure 5.32: Normalized electric field verses delay time multiplied by pressure in air. Curve for volume breakdown (blue) from Gould and Roberts compared to data collected on current test setup (black circles $3 \mathrm{MW}$ or $18 \mathrm{kV} / \mathrm{cm}$ pulse and black triangles for $4.5 \mathrm{MW}$ or $25 \mathrm{kV} / \mathrm{cm}$ pulse). The red line represents a curve fit of the current data. [15]

The $\mathrm{E}_{\text {eff }} / \mathrm{p}$ vs. $\mathrm{p}^{*} \tau$ values of the current setup also lie about a single curve although it lies higher than that of Gould and Roberts. There are several possible explanations for this shift, including the lack of electrodes, or any other metallic surfaces, for injection of electrons into the gap, as well as the surface itself acting as a loss mechanism and thus increasing the delay times. Additionally, the experiments performed by Gould and Roberts were conducted in homogeneous electric fields with geometry dependence in only one direction, while in the current setup there are strongly inhomogeneous fields with a geometry dependence in three dimensions. Finally, Gould and Roberts used the $\mathrm{CW}$ breakdown field to normalize the pulse to an ideal square pulse, which is not 
possible in the present case as the $\mathrm{CW}$ flashover fields are not known. The combination of all of these factors is believed to be responsible for the vertical shift of the curve [15].

Having acquired data sets for surface flashover in both air and nitrogen from the HPM setup, the simulation is initialized with the same parameters as the experiment and run to determine the simulated delay times. Figure 5.33 shows a data set collected in an air environment under various excitations and pressures along with the corresponding results from the simulation. It is evident that a strong correlation exists between the calculated and the actual data in regards to the delay time. However, as shown previously, a complete correlation of experimental to calculated data has not been reached due to the differences in the arc channel formation path. 


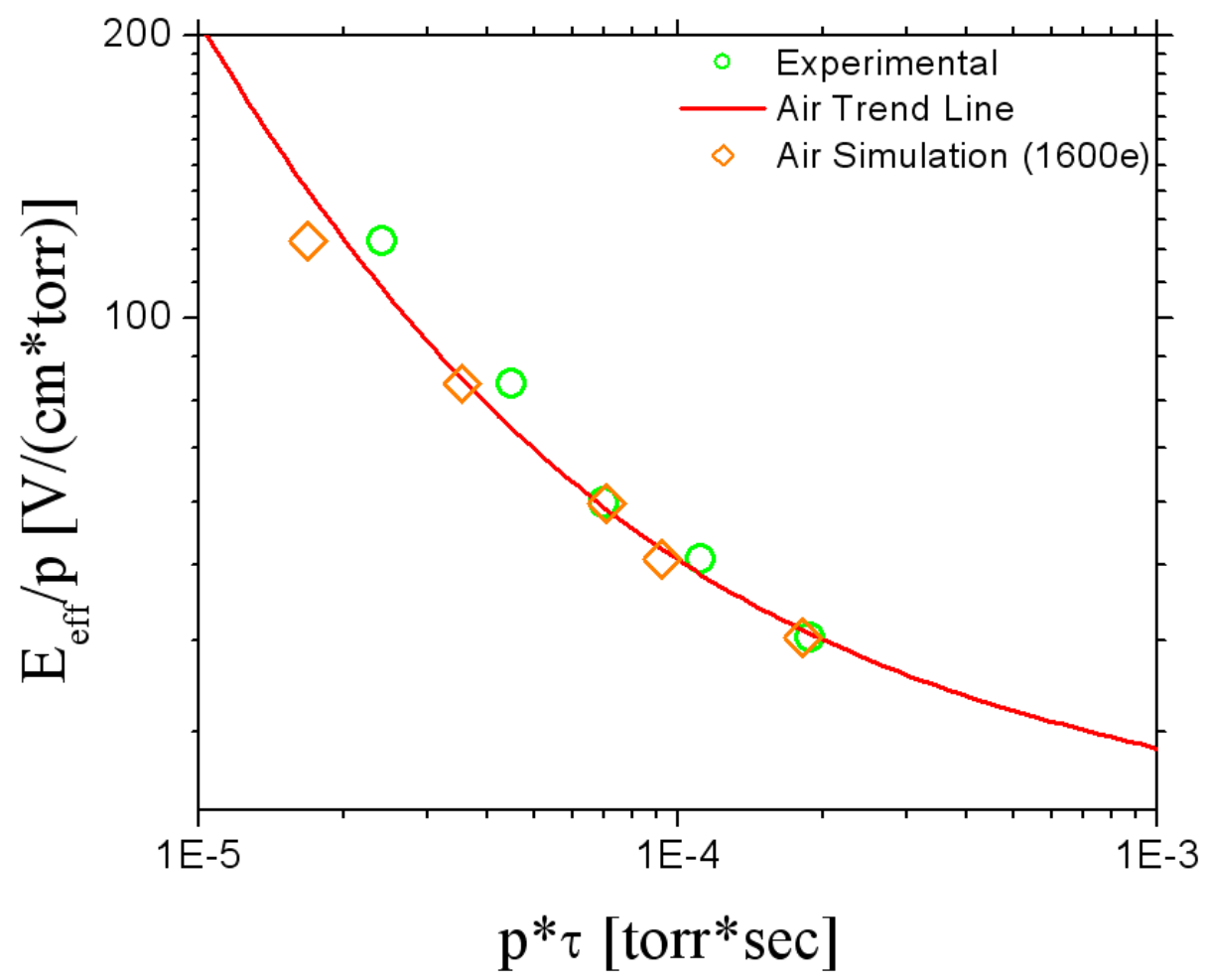

Figure 5.33: Normalized electric field verses delay time multiplied by pressure in air. The graph shows data collected on the current test setup (green circles), simulated data (orange diamonds), and a curve fit of the all experimental data in air (red line)

A similar comparison is done in a pure nitrogen environment with the result shown in Figure 5.34. In this figure there are two sets of simulation data presented, the first is a simulation series run with 1600 initial electron and the second run with 16 initial electrons. As can be seen neither curve generated by the simulation corresponds well with the experimental data, however, the nitrogen curves do lie above the air curve just as the experimental data dictates. As the number of initial electrons is reduced an increased correlation is observed. While there are no firm numbers available in the literature as to the free electron concentration in a pure nitrogen environment, it is believed to be very small. As a result, the increased delay times observed in the experiment might be the 
result of a large statistical delay time until a primary electron passes into the high field region and begins the electron multiplication process. Experiments involving the UV illumination of the surface during the applied HPM pulse are currently being carried out and are believed to yield some insight in this matter.

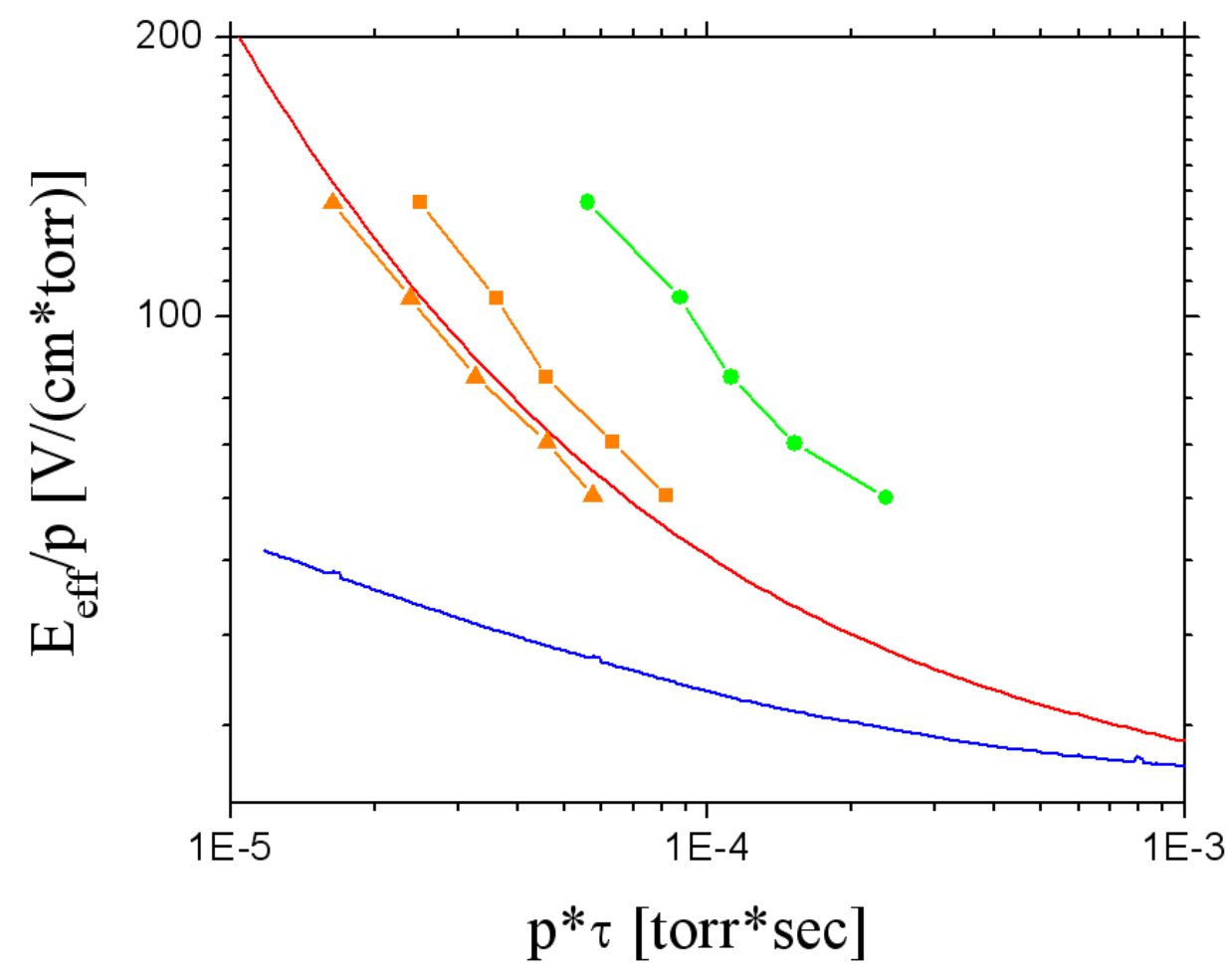

Figure 5.34: Normalized electric field verses delay time multiplied by pressure in nitrogen. The graph shows volume breakdown curve from Gould and Roberts (blue line), data collected on current test setup (green circles), simulated data with 1600 initial electrons (orange triangles), simulated data with 16 initial electrons (orange squares), and a curve fit of the all experimental data in air (red line)

As stated above, Gould and Roberts used a normalization method on the experimental HPM pulse to achieve an equivalent ideal square pulse. This is not possible under the current experimental setup, but can be done within the simulation. Two series of data were collected, one in air and one in pure nitrogen, with the pressure held constant while 
the field strength is varied. As seen in Figure 5.35, the data in an air environment shows a strong correlation with the data collected in air by Gould and Roberts. In addition, the nitrogen data lies above the air which correlates with previous findings. This correlation shows the importance of the normalization applied by Gould and Roberts, and the need for its careful consideration in analysis of future experimental results.

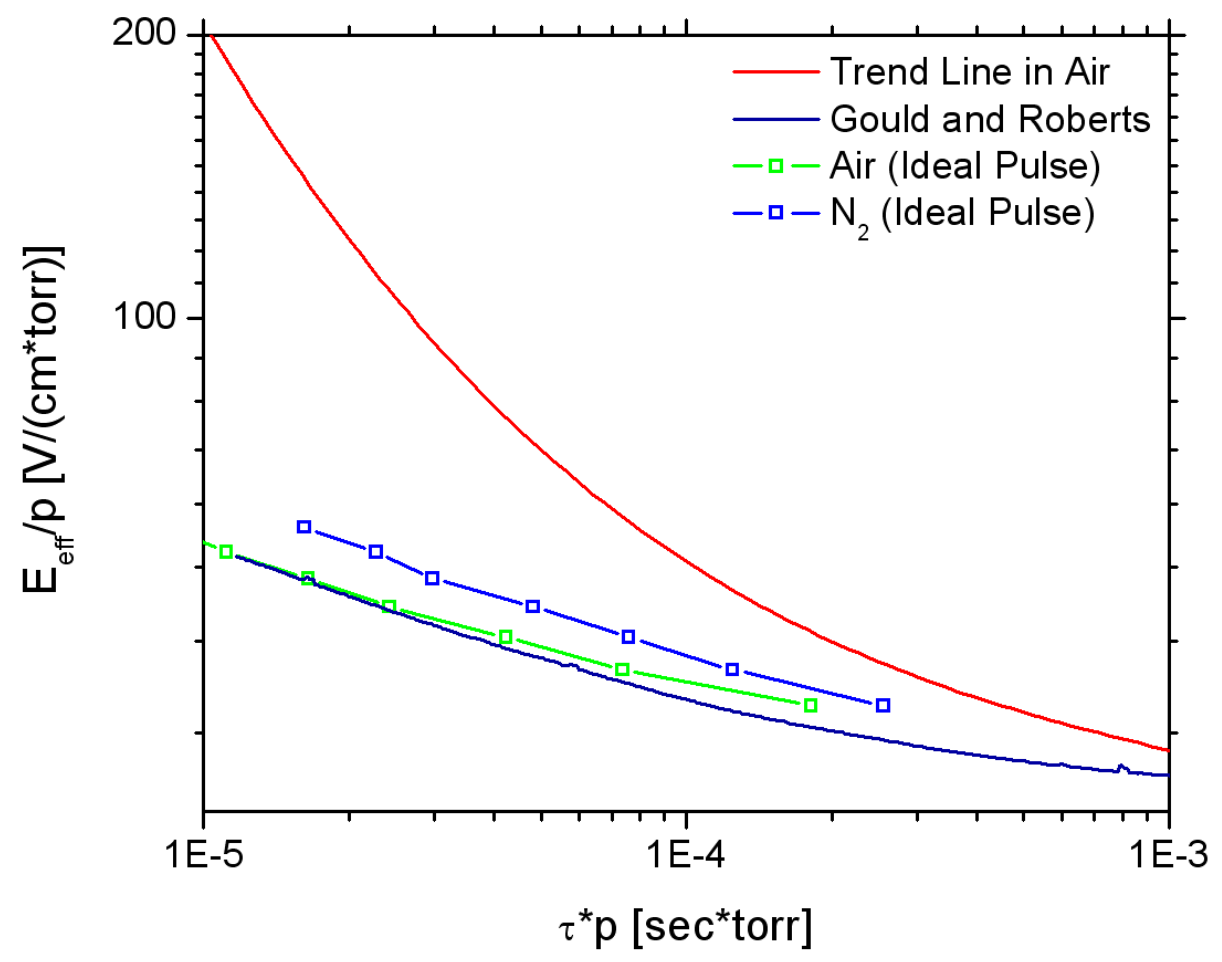

Figure 5.35: Normalized electric field verses delay time multiplied by pressure in both air (green squares) and nitrogen (blue squares) environments. The graph also shows volume breakdown curve from Gould and Roberts (dark blue line), and a curve fit of all the experimental data in air (red line)

\subsubsection{Electron Energy Distribution}

In addition to the number and position of the electrons, the simulation also records the energy distribution of the electrons at each time step. The distribution changes with time as the electrons are accelerated by the alternating field and undergo various collisions, as 
can be seen in Figure 5.36 below. As a result it is necessary to average the electron energy distribution function, or EEDF, over several periods to obtain an average EEDF.

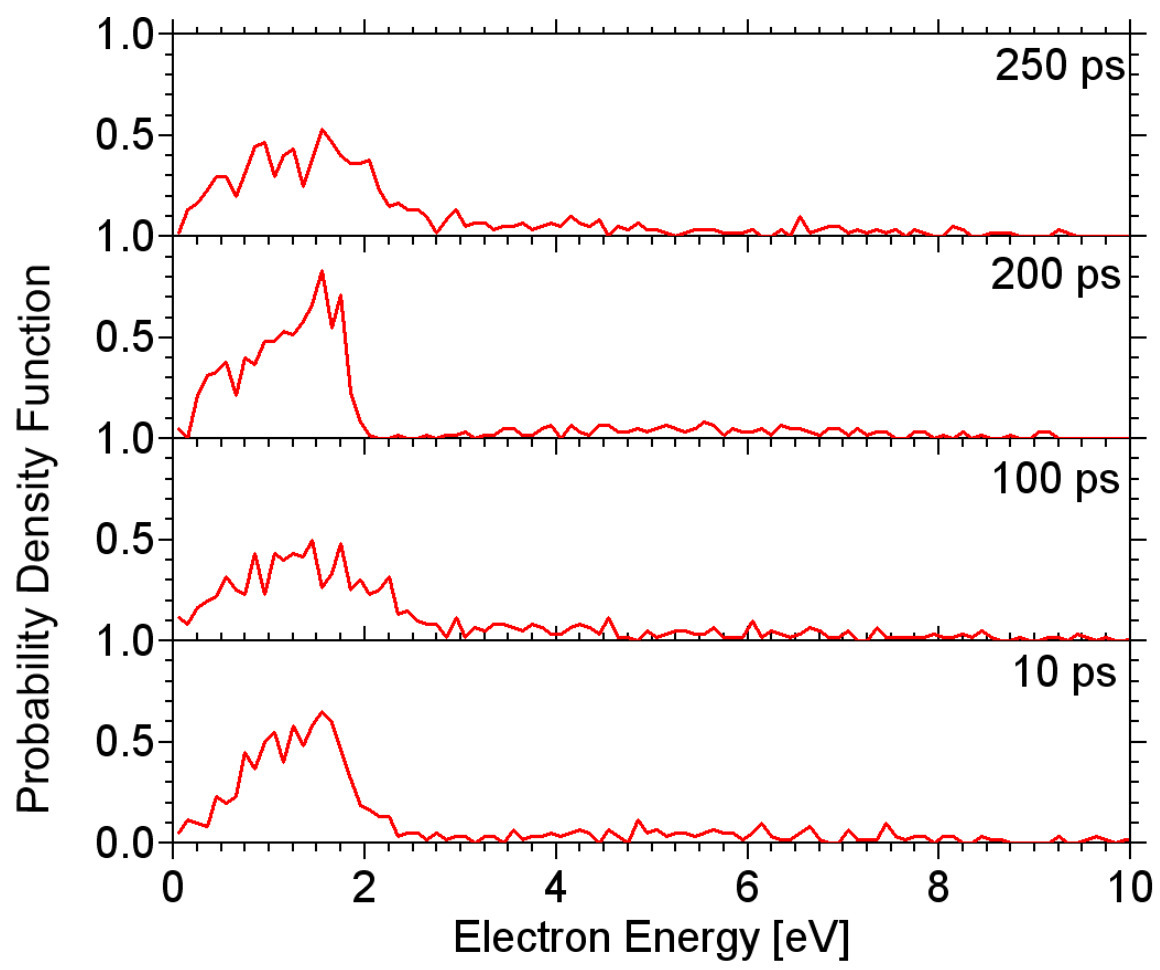

Figure 5.36: Snap shot of the electron energy distribution function at several time steps in an air environment at 125 torr, with a microwave period of $350 \mathrm{~ns}$.

In order to provide an accurate comparison with the EEDF generated for steady state conditions by an alternate EEDF program, created by Dr. Napartovich as previously discussed with the 1D results, the periods averaged have a zero net gain of electrons. As can be seen in Figure 5.37 and Figure 5.38, the EEDF's share some similarities yet important differences remain. It should be noted that the rough appearance of the simulated EEDF in nitrogen is due to the small number of electrons present at the chosen steady state conditions, around 20, as opposed to air with around 1600. In both and air and nitrogen case the EEDF's share a similar drop off point around $2 \mathrm{eV}$, and in the case 
of air, the "tail" of the distribution is also quite similar. This is important due to the tails, or higher energy electrons, contribution to the ionization process. Stark differences in the lower energy ranges, less than $2 \mathrm{eV}$, include the overall height of the distribution and the lack of ramp up starting at $0 \mathrm{eV}$ in the alternate EEDF. It is difficult to draw too many conclusions from these differences as the initial conditions and simplifying assumption made by Dr. Napartovich are currently unknown. Additionally, the area under any probability density function should be 1 . However, this is not the case for the alternate EEDF. Even with these shortcomings, the comparison provides a rough check on the validity of the program.

Other calculations of the EEDF, done by Kunhardt and Tzeng, bear a closer resemblance to the current simulation result, especially in the energy range approaching 0 $\mathrm{eV}[50,57]$. The calculations were carried out using a direct simulation method similar to the one employed currently. These results further validate the output of the current MC code. 


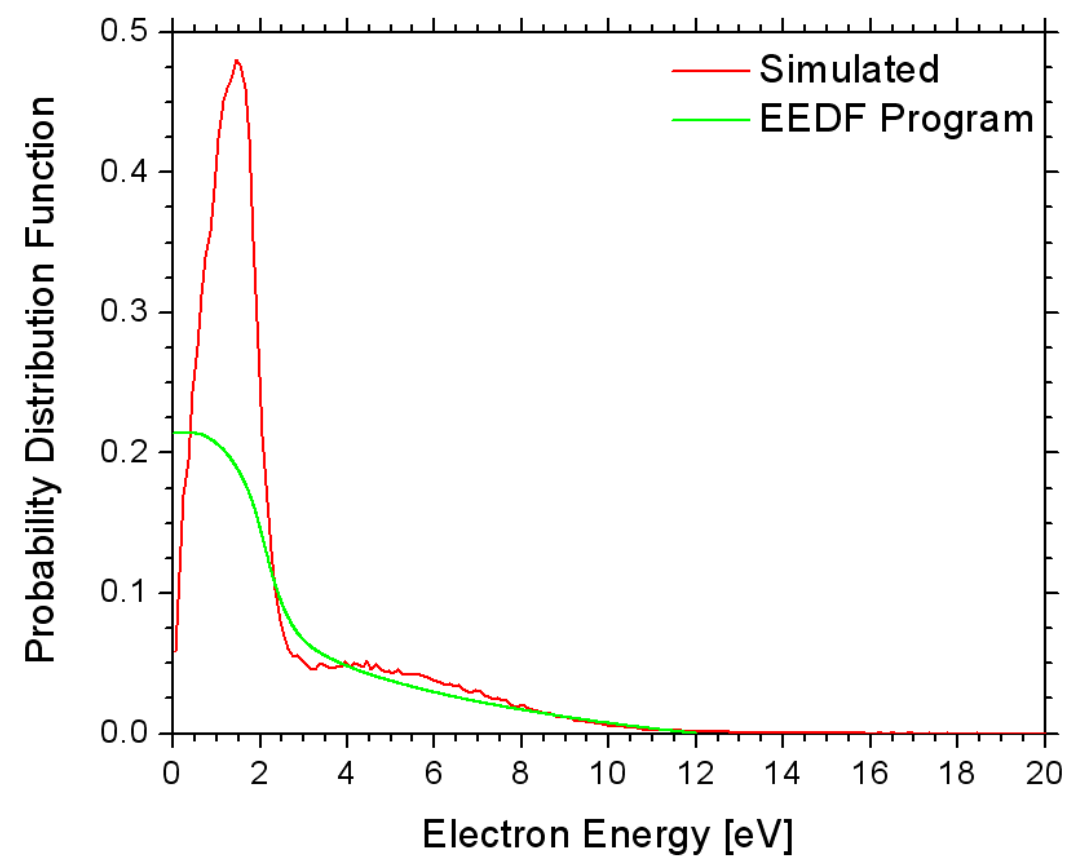

Figure 5.37: Comparison of EEDF's for an air environment generated by the simulation and by an alternate EEDF program, the simulation EEDF is averaged over 2 periods with zero net electron gain

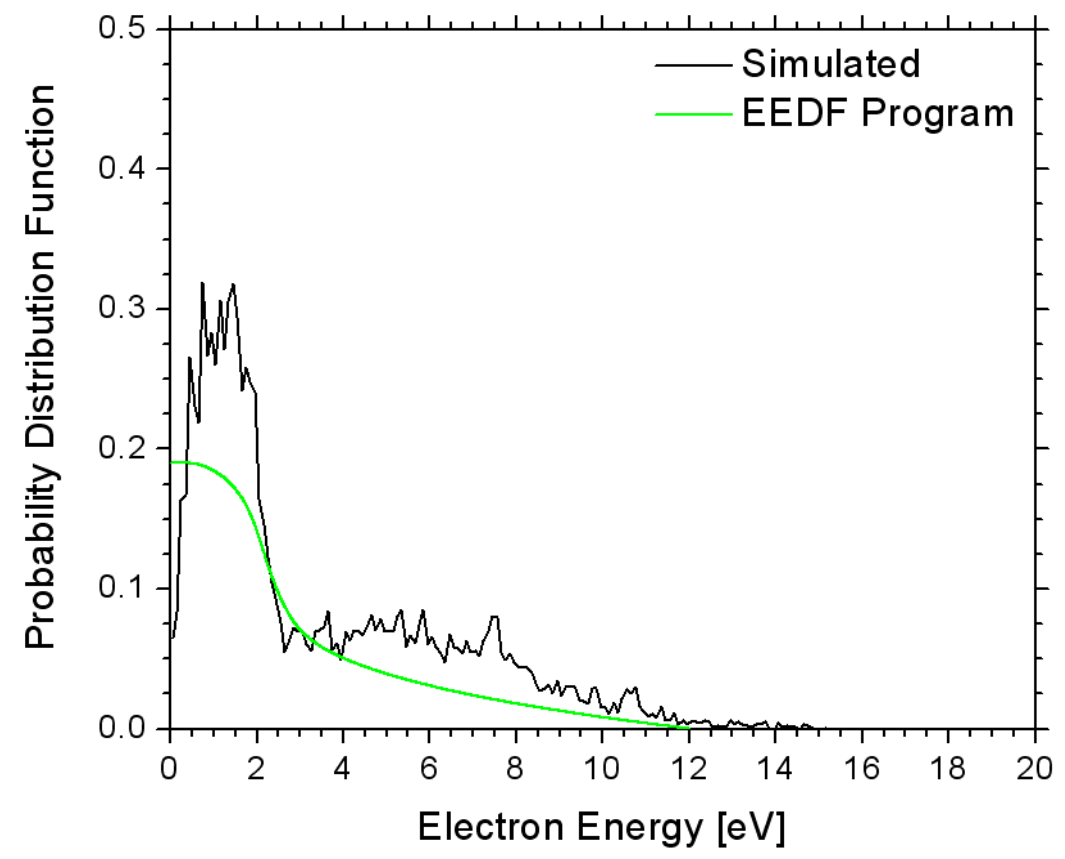

Figure 5.38: Comparison of EEDF's for a nitrogen environment generated by the simulation and by an alternate EEDF program, the simulation EEDF is averaged over 2 periods with zero net electron gain 
It is also useful to compare the EEDF's generated by the simulation for both environments, as seen in Figure 5.39. The distributions in both environments show a similar slope at low energies, below $0.2 \mathrm{eV}$, and a similar drop off around $2 \mathrm{eV}$. The main difference in the distributions occurs in the low energy range before the drop off and in the tail of the distributions. This is attributed to several differences in the cross sections for oxygen and nitrogen. The larger concentration of higher energy electrons, or the larger tail, of the nitrogen distribution is due to the fact that nitrogen has a higher ionization energy that oxygen. This allows more electrons to reach higher energies before losing their energy to ionization. It also helps explain the smaller number of electrons at low energies in nitrogen as compared to air. The tail of the air distribution is also lower due to the scavenging of electrons, in the 4 to $9 \mathrm{eV}$ range, by the electronegative oxygen.

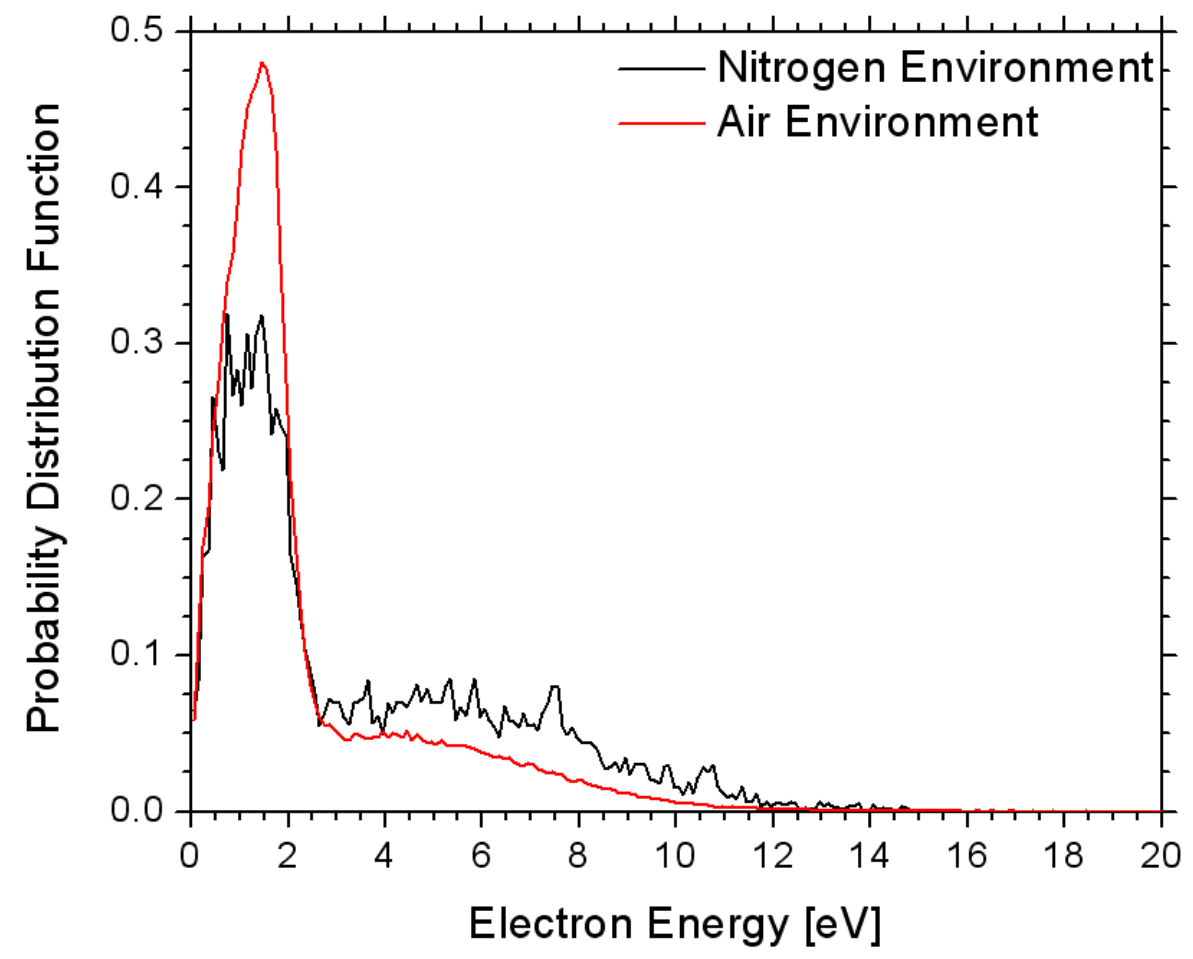

Figure 5.39: Comparison of EEDF's for air and nitrogen environments generated by the simulation, the EEDF's are averaged over 2 periods with zero net electron gain 


\section{CHAPTER 6}

\section{CONCLUSIONS}

Similarities do exist between unipolar and HPM surface flashover at atmospheric conditions. This is particularly evident with respect to the suspected role of UV in the arc channel formation, leading a discharge channel in an $\mathrm{N}_{2}$ environment to liftoff of the surface in either unipolar or HPM excitation. UV radiation externally applied to the surface causes the discharge to follow the surface in all observed cases. Additionally, the total flashover delay times are reduced by UV application, mostly due to the elimination of the statistical time delay. There is also a correlation of the effects of humidity, with an increase in relative humidity decreasing the flashover voltage in the DC unipolar case and lowering the delay times in the HPM case. In both cases the moisture is believed to influence the flashover by creating a thin layer of moisture that is easily ionized, yielding more free electrons in the high field region.

There are important differences between flashover under unipolar and HPM excitation as well. It is shown by utilizing emission spectroscopy in the range from 180 to $800 \mathrm{~nm}$ that the HPM flashover discharge has a lower plasma temperature than the arc of the unipolar flashover. This temperature differential causes significant changes in the type of excited and ionized molecules.

The newly created Monte Carlo simulation program has successfully demonstrated its ability to reproduce results seen on both the present HPM setup and previous research done by Gould and Roberts. Sensitivity testing of the program showed a strong 
dependence on the collision cross sections while almost no dependence on the random process of placing electrons within the volume, further supporting the validity of the program. The simulation has also provided insight into electron cloud formation under oscillation fields, as well as the relatively limited role of electron surface impacts.

While the present MC simulation code provided results similar to those obtained experimentally, it is clear that there are still physical processes of flashover that have not been accounted for, such as photoemission of electrons from the surface. Given the inability of the code to accurately predict the path of arc channel formation, these processes are believed to play a key role in the determination of the arc path. Of the processes accounted for, the dominant role of the electronegative oxygen molecule is confirmed as it has a noticeable effect on the arc channel formation in the high field region. It has also demonstrated the need for more information about the environment where the arc is initiated, including parameters such as the initial electron density.

Given the MC code's flexibility with respect to collision cross sections it should be possible to expand the cross section database to include other commonly used gases, such as argon, hydrogen, and $\mathrm{SF}_{6}$. These simulations can then be run over a wide array of conditions, varying gas types and mixtures as well as pressure and temperature. An added advantage is the MPI protocol, which allows the simulation to be run on as big a cluster as can be accessed. Combined with the ability to add and update the all important electron collision cross sections, the developed code should remain a valuable tool in the study of HPM flashover into the future. 


\section{REFERENCES}

[1] J. Krile, A. Neuber, J. Dickens, and H. Krompholz, "DC and Pulsed Dielectric Surface Flashover at Atmospheric Pressure," IEEE Transactions on Plasma Science, Vol. 33, No. 4, pp. 1149-1154, August 2005.

[2] H. Miller, "Surface Flashover of Insulators," IEEE Transactions on Electrical Insulation, Vol. 24, No. 5, pp. 765-786, October 1989.

[3] R. E. Jorgenson, L. K. Warne, et al., "Effect of Dielectric Photoemission on Surface Breakdown: An LDRD Report," Sandia Report SAND2003-3044, June 2003.

[4] A. Neuber, L. Laurent, Y. Y. Lau, and H. Krompholz, "Windows and RF Breakdown," in High-Power Microwave Sources and Technologies, Chap. 10, pp. 325-375, Ed. by R. J. Barker and E. Schamiloglu, IEEE Press, London, 2001.

[5] D. Hemmert, A. Neuber, J. Dickens, H. Krompholz, L. Hatfield, and M. Kristiansen, "Microwave Magnetic Field Effects on High-Power Microwave Window Breakdown," IEEE Transactions on Plasma Science, Vol. 28, No. 3, pp. 472-477, June 2000.

[6] A. Neuber, D. Hemmert, H. Krompholz, L. Hatfield, and M. Kristiansen, "Initiation of High Power Microwave Dielectric Interface Breakdown," Journal of Applied Physics, Vol. 86, No. 3, pp.1724-1728, August 1999.

[7] F. Hegeler, G. Masten, and H. Krompholz, "Current, Luminosity, and X-Ray Emission in the Early Phase of Dielectric Surface Flashover in Vacuum," IEEE Transactions on Plasma Science, Vol. 21, No. 2, pp. 223-227, April 1993.

[8] F. Hegeler, H. Krompholz, L. Hatfield, and M. Kristiansen, "Insulator Breakdown in a Simulated Low Earth Orbit Environment," 10th IEEE Pulsed Power Conference, Albuquerque, NM July 10-13, 1995.

[9] A. Neuber, H. Krompholz, and L. Hatfield, "Dielectric Surface Flashover at Cryogenic Temperatures," Conference on Electrical Insulation and Dielectric Phenomena, pp. 575-578, Minneapolis, MN, October 19-20, 1997.

[10] A. Neuber, L. Laurent, Y. Lau, and H. Krompholz, "Windows and RF Breakdown," High-Power Microwave Sources and Technologies, IEEE Press, New York, 2001.

[11] S. Calico, "High Power Microwave Breakdown of Dielectric Interfaces," Dissertation for the Degree of Doctor of Philosophy in Electrical Engineering, Texas Tech University, Lubbock, Texas, 1991. 
[12] L. L. Hatfield, E. R. Boerwinkle, G. Leiker, R. Korzekwa, M. Lehr, and M. Kristiansen, "Methods of Increasing the Surface Flashover Potential in Vacuum," IEEE Transactions on Electrical Insulation, Vol. 24, No. 6, pp. 985-990, December 1989.

[13] A. Neuber, J. Dickens, D. Hemmert, H. Krompholz, L. L. Hatfield, and M. Kristiansen, "Window Breakdown Caused By High-Power Microwaves," IEEE Transactions on Plasma Science, Vol. 26, No. 3, pp. 296-303, June 1998.

[14] J. Krile, G. Edmiston, K. Morales, A. Neuber, H. Krompholz, and M. Kristiansen, "Similarities of Dielectric Surface Flashover under Atmospheric Conditions for Pulsed Unipolar and RF Excitation," Laser Physics, vol. 16, no. 1, pp. 194-201, 2006.

[15] G. Edmiston, "High Power Microwave Window Flashover at Atmospheric Pressures," Thesis for the Degree of Master of Science in Electrical Engineering, Texas Tech University, Lubbock, Texas, 2005.

[16] E. Nasser, Fundamentals of Gaseous Electronics, Wiley-Interscience, New York, NY, 1971.

[17] Y. Raizer, Gas Discharge Physics, Springer-Verlag, New York, NY, 1991.

[18] J. M. Meek and J. D. Craggs, Electrical Breakdown of Gases, John Wiley and Sons, 1978.

[19] R. H. Good, and W. W. Muller, "Field Emission," Encyclopedia of Physics, Vol. XXI, Springer, Berlin, pp. $176-231,1956$.

[20] R. H. Fowler and L. W. Nordheim, "The Effect of the Image Force on the Emission and Reflexion of Electrons by Metals," Proceeding of the Royal London Society A, Vol. 121, No. 788, pp. 626-639, December 1928.

[21] M. Fujihira, and H. Inokuchi, "Photoemission from Polyethylene," Chemical Physics Letters, Vol. 17, No. 4, pp. 554-556, December 1972.

[22] J. Tom, H. A. Verhaart, A. L. Verhage, and C. S. Vos, "Photo-emission of Charged Insulators in Insulating Gases," Proceedings of the 2nd International Conference on Conduction and Breakdown in Solid Dielectrics, pp. 301-307, Erlangen, 1986.

[23] A. A. Guzhov, and Yu. A. Shuba, "The Phototemission of Some Massive Insulators in the Vacuum Ultraviolet," Optical Technology, Vol. 38, No. 4, pp. 198-199, April 1971. 
[24] G. F. Dionne, "Origin of Secondary-Electron-Emission Yield-Curve Parameters," Journal of Applied Physics, Vol. 46, No. 8, pp. 3347-3351, August 1975.

[25] National Institute of Standards and Technology, "Electron Impact Cross Sections for Ionization and Excitation,” January 2006, http://physics.nist.gov/PhysRefData/Ionization/molTable.html/.

[26] G. J. Schulz, "Cross Sections and Electron Affinity for $\mathrm{O}^{-}$Ions from $\mathrm{O}_{2}, \mathrm{CO}$, and $\mathrm{CO}_{2}$ by Electron Impact," Physical Review, Vol. 128, No. 1, pp. 178-186, October 1962.

[27] A. V. Phelps, and L. C. Pitchford, "Anisotropic Scattering of Electrons by $\mathrm{N}_{2}$ and Its Effects on Electron Transport: Tabulations of Cross Section and Results," Report No. 26, University of Colorado, May 1985.

[28] A. V. Phelps, "Tabulations of Collision Cross Sections and Calculated Transport and Reaction Coefficients for Electron Collisions with $\mathrm{O}_{2}$," Report No. 28, University of Colorado, Sept. 1985.

[29] D. C. Cartwright, "Electron Impact Excitation of the Electronic States of $\mathrm{N}_{2}$. II. Integral Cross Sections at Incident Energies from 10 to $50 \mathrm{eV}$," Physical Review A, Vol. 16, No. 3, pp. 1041-1051, September 1977.

[30] Pevgov et al., Plasma Chemistry Reactions, L. S. Ed. Polak, Moscow 1979.

[31] R. Islamov et al., P. N. Lebedev Physical Institute of the Russian Academy of Sciences, Vol. 169, Moscow 1977.

[32] S. C. Brown, Introduction to Electrical Discharges in Gases, John Wiley \& Sons Inc., New York, 1966.

[33] R. Papoular, Electrical Phenomena in Gases, American Elsevier Pub. Co. Inc., New York, 1965.

[34] G. J. Townsend, "The Conductivity Produced in Gases by the Aid of Ultraviolet Light,” Philosophical Magazine, Vol. 6, pp. 389, April 1903.

[35] J. S. Townsend, Electrons in Gases, Hutchinson, London, 1947.

[36] M. Akyuz, "Positive Streamer Discharges in Air and Along Insulating Surfaces: Experiment and Simulation," Dissertation for the Degree of Doctor of Technology in Engineering Physics, Uppsala University, Uppsala, Sweden, 2002. 
[37] H. Raether, "Uber den Aufbau von Gasentladungen. I," Zeitschrift für Physik, Vol. 117, pp. 375-399, 1941.

[38] L. B. Loeb and J. M. Meek, "The Mechanism of Spark Discharge in Air at Atmospheric Pressure. I," Journal of Applied Physics, Vol. 11, No. 6, pp. 438-447, June 1940.

[39] R. A. Anderson, and J. P. Brainard, "Mechanism of Pulsed Surface Flashover Involving Electron-Stimulated Desorption," Journal of Applied Physics, Vol. 51, No. 3, pp.1414-1421, March 1980.

[40] A. Neuber, M. Butcher, L. Hatfield, and H. Krompholz, "Electric Current in DC Surface Flashover in Vacuum," Journal of Applied Physics, Vol. 85, No. 6, pp. 3084-3091, March 1999.

[41] A. Pedersen, "On the Electrical Breakdown of Gaseous Dielectrics," IEEE Transactions on Electrical Insulation, Vol. 24, No 5, pp. 721-739, October 1989.

[42] A. D. MacDonald, Microwave Breakdown in Gases, John Wiley \& Sons, New York, 1966.

[43] M. A. Herlin and S. C. Brown, "Breakdown of a Gas at Microwave Frequencies," Physical Review, Vol. 74, No. 3, pp. 291-296, August 1948.

[44] L. Gould and L. W. Roberts, "Breakdown of Air at Microwave Frequencies," Journal f Applied Physics, Vol. 27, No. 10, pp. 1162-1170, October 1956.

[45] A. D. MacDonald, D. U. Gaskell, and H. N. Gitterman, "Microwave Breakdown in Air, Oxygen and Nitrgen," Physical Review, Vol. 130, No. 5, pp. 1841-1850, June 1963.

[46] C. A. Sullivan, W. W. Delstler, J. Rodgers, and Z. Segalov, "Short-Pulse High Power Microwave Propagation in the Atmosphere," Journal of Applied Physics, Vol. 63, No. 11, pp. 5228-5232, June 1988.

[47] J. Krile, "Physics of Dielectric Surface Flashover at Atmospheric Pressures," Thesis for the Degree of Master of Science in Electrical Engineering, Texas Tech University, Lubbock, Texas, 2003.

[48] H. Krompholz, K. Schoenbach, and G. Schaefer, "Transmission Line Current Sensor," Proceedings of the ITMC-IEEE Instrumentation and Measurement Technology Conference, pp.224-227, March 20-22, 1985. 
[49] A. P. Napartovich, "EEDF: Program for the Computation of the Electron Energy Distribution Function," State Science Center Russian Federation at Troitsk Institute for Innovation Fusion Research, Troitsk, Russia, 1993.

[50] E. E. Kunhardt and Y Tzeng, "Monte Carlo Technique for Simulating the Evolution of an Assembly of Particles Increasing in Number," Journal of Computational Physics, Vol. 67, No. 2, pp. 279-289, December 1986.

[51] C. K. Birdsall, "Particle-in-Cell Charged-Particle Simulations, Plus Monte Carlo Collisions With Neutral Atoms, PIC-MCC," IEEE Transactions on Plasma Science, Vol. 19, No. 2, pp. 65-85, April 1991.

[52] M. J. Brennan, "Optimization of Monte Carlo Codes Using Null Collision Techniques for Experimental Simulation at Low E/N," IEEE Transactions on Plasma Science, Vol. 19, No. 2, pp. 256-261, April 1991.

[53] S. L. Lin and J. N. Bardsley, "Monte Carlo Simulation of Ion Motion in Drift Tubes," Journal of Chemical Physics, Vol. 66, No. 2, pp. 435-445, January 1977.

[54] H. R. Skullerud, "The Stochastic Computer Simulation of Ion Motion in a Gas Subjected to a Constant Electric Field," Journal of Physics D, Vol. 1, No. 11, pp. 1567-1568, November 1968.

[55] H. R. Skullerud, "Monte-Carlo Investigations of the Motion of Gaseous Ions in Electrostatic Fields," Journal of Physics B, Vol. 6, No. 4, pp. 728-742, April 1973.

[56] R. E. Jorgenson, L. K. Warne, and E. E. Kunhardt, "Lightning Induced Arcing: An LDRD Report," Sandia Report SAND2000-3044, Dec. 2000.

[57] E. E. Kunhardt, Y. Tzeng, and J. P. Boeuf, "Stochastic Development of an Electron Avalanche," Physical Review A, Vol. 34, No. 1, pp. 440-449, July 1986.

[58] K. P. Morales, J. T. Krile, A. A. Neuber, and H. G. Krompholz, "Pulsed Dielectric Surface Flashover in Atmospheric Conditions," submitted to IEEE Transactions on Dielectrics and Electrical Insulation, September 2005.

[59] E. Hippauf, "Influence of Water on the Flashover Strength of Insulators," Zeitschrift für Physik, Vol. 82, pp. 803-814, 1933.

[60] L. G. Christophorou and L. A. Pinnaduwage, "Basic Physics of Gaseous Dielectrics," IEEE Transactions on Electrical Insulation, Vol. 25, No. 1, pp. 55-74, February 1990. 